\title{
Entwurfsaerodynamische Studien an Tragflügelkonfigurationen im Hochgeschwindigkeitsbereich mit evolutionären Algorithmen
}

\author{
Dissertation \\ zur Erlangung des Doktorgrades \\ der Mathematisch-Naturwissenschaftlichen Fakultäten \\ der Georg-August-Universität zu Göttingen
}

vorgelegt von

Michael Klein

aus Hamburg

Göttingen 2000 
D 7

Referent: Prof. Dr. H. Eckelmann

Korreferent: Prof. Dr. H. Sobieczky

Tag der mündlichen Prüfung: 20.6.2000 


\section{INHALTSVERZEICHNIS}

Liste der verwendeten Bezeichungen $\quad$ v

1. Einleitung 1

2. Motivation und Zielsetzung 5

$\begin{array}{ll}\text { 3. Die Evolutionären Methoden } & 7\end{array}$

3.1 Motivation zur Nutzung Evolutionärer Methoden $\ldots \ldots \ldots \ldots \ldots \ldots \ldots$

3.2 Eigenschaften der Evolutionären Methoden $\ldots \ldots \ldots \ldots \ldots \ldots \ldots \ldots \ldots$

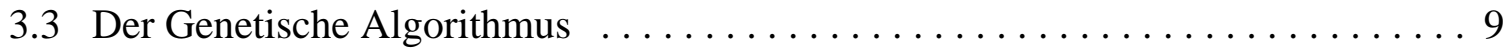

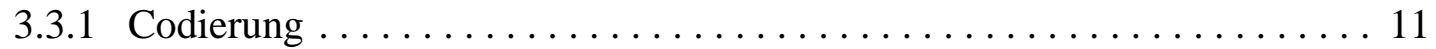

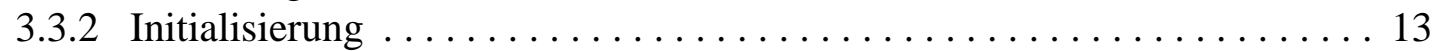

3.3 .3 Evaluation ................................. 13

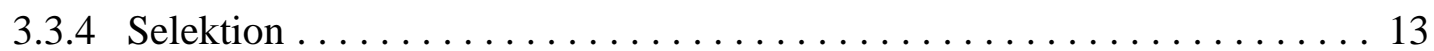

3.3.5 Rekombination ............................... 13

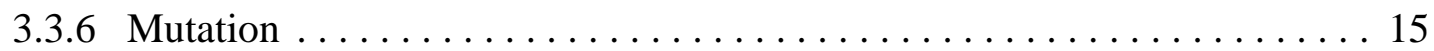

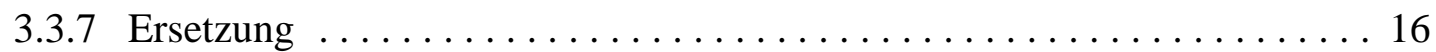

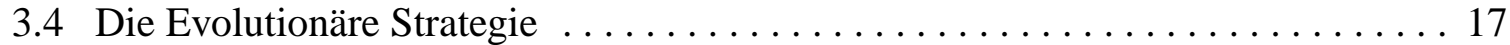

3.5 Einfachoptimierung mit Evolutionären Methoden $\ldots \ldots \ldots \ldots \ldots \ldots \ldots \ldots$

3.6 Mehrfachoptimierung mit Evolutionären Methoden $\ldots \ldots \ldots \ldots \ldots \ldots \ldots$

4. Geometrische Grundlagen $\quad 24$

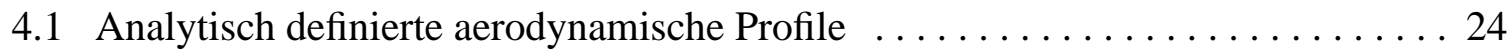

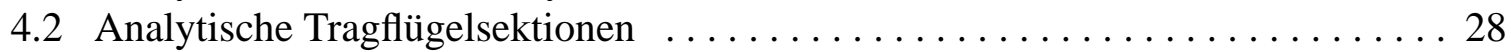

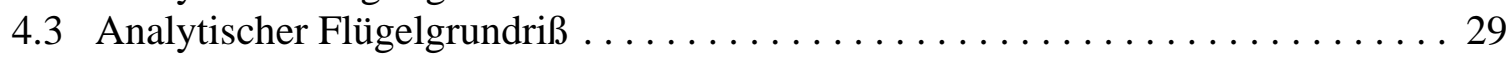

5. Physikalische Grundlagen 31

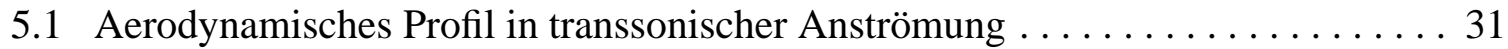

5.2 Numerische Simulation der Strömung . . . . . . . . . . . . . . . . 33

5.3 Druckverhältnisse am Profil . . . . . . . . . . . . . . . . . 34

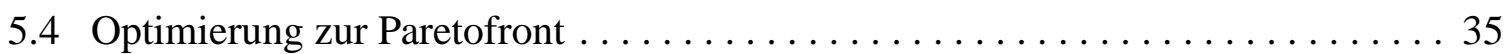

5.5 Berechnung der Paretofronten auf einem Parallelcluster von Rechnern . . . . . 36

6. Ergebnisse aus der Anwendung der evolutionären Methoden 38

6.1 Die Wirkungsweise des Genetischen Algorithmus $\ldots \ldots \ldots \ldots \ldots \ldots \ldots$

6.2 Anwendung des Genetischen Algorithmus auf einsektionale Profile . . . . . . . . 40

6.3 Erweiterung auf mehrsektionale Profile verschiedener Funktionen . . . . . . . . . 44

6.4 Die Paretofronten mehrsektionaler Profile konstanter Dicke . . . . . . . . . . 51

6.5 Qualität mehrsektionaler Profile konstanter Dicke . . . . . . . . . . . . . . . 54

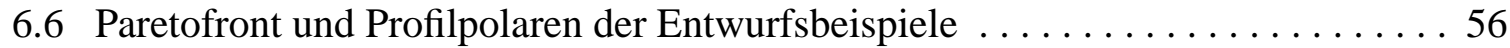

6.7 Die Parameter der Sektionen auf der Paretofront . . . . . . . . . . . . . . . . 58

6.8 Paretofront aus Navier-Stokes-Verfahren . . . . . . . . . . . . . . . . . . . . 63

6.9 Anwendung der Evolutionären Strategie auf eine existierende Paretofront . . . . 65 


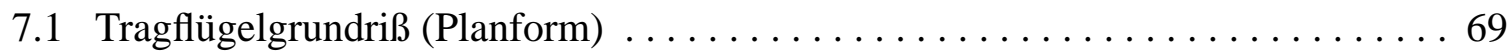

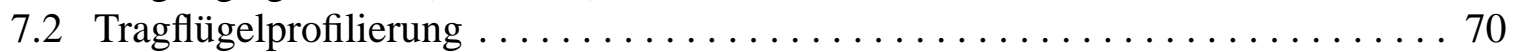

7.2.1 Elliptischer Tragflügel mit konstanter Profilierung $\ldots \ldots \ldots \ldots \ldots \ldots 70$

7.2 .2 Tragflügelgestaltung durch optimierte Profile $\ldots \ldots \ldots \ldots \ldots \ldots \ldots . \ldots 71$

7.2.3 Tragflügelgestaltung durch Genetischen Algorithmus . . . . . . . . . . 73

8. Ergebnisse für Tragflügelkonfigurationen im Überschall 81

8.1 Allgemeine Betrachtungen zur Auslegung einer Überschallkonfiguration . . . . 81

8.2 Auslegung einer Überschallkonfiguration nach der linearen Theorie . . . . . . . . 82

8.3 Beispiel einer Konfiguration aus der klassischen linearen Überschallheorie . . . . 84

8.4 Auslegung einer Überschallkonfiguration durch den Genetischen Algorithmus . 86

9. Zusammenfassung $\quad 89$

$\begin{array}{ll}\text { Anhang A Basisfunktionen zur Geometriedefinition } & 91\end{array}$

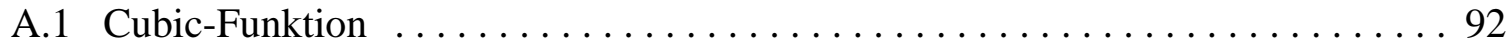

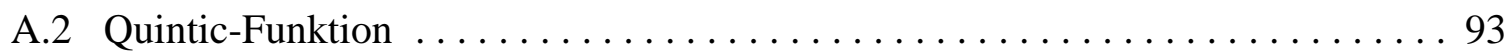

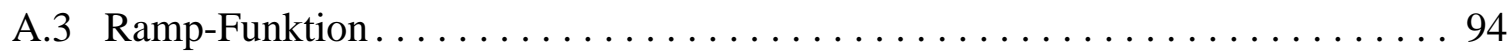

Literaturverzeichnis $\ldots \ldots \ldots \ldots \ldots \ldots \ldots \ldots \ldots \ldots \ldots \ldots$ 


\section{Liste der verwendeten Bezeichnungen}

\section{Strömungsmechanische Größen und Bezeichnungen}

$c_{p}$

$c_{v}$

$c$

$c_{p}=\frac{p-p_{\infty}}{\frac{\rho}{2} q_{\infty}^{2}}$

$c_{\mathrm{p}^{*}}$

$c_{l}$

$c_{d}$

$c_{m}$

$c_{f}$

D

$g$

$L$

$l$

$M_{\infty}$

$p$

$p_{\infty}$

$q=\sqrt{\left(u^{2}+v^{2}\right)}$

$q_{\infty}$

$R e=\frac{q l}{v}$

$S$

$S_{f}$

$s_{1}$

$s_{2}$

$e_{1}$ spezifische Wärme bei konstantem Druck

spezifische Wärme bei konstantem Volumen

Schallgeschwindigkeit

Druckkoeffizient

kritischer Druckwert bei Schalldurchgang

Auftriebsbeiwert

Widerstandsbeiwert

Momentenbeiwert

Reibungsbeiwert

Gesamtwiderstand

Funktionskennzahl

Gesamtauftrieb

Bezugslänge

Machzahl in der Anströmung

Druck

Druck (Anströmung)

Geschwindigkeitsbetrag

Geschwindigkeit (Anströmung)

Reynoldszahl

Entropie

Referenzfläche für Oberflächenreibung

Funktionsparameter

Funktionsparameter

Funktionsparameter 


$\begin{array}{ll}e_{2} & \text { Funktionsparameter } \\ u & \text { Geschwindigkeitskomponente in x-Richtung (Strömungsrichtung) } \\ v & \text { Geschwindigkeitskomponente in y-Richtung } \\ w & \text { Geschwindigkeitskomponente in z-Richtung } \\ x, y, z & \text { kartesische Koordinaten }\end{array}$

\section{Griechische Symbole}

$\alpha$

Anstellwinkel des Tragflügelprofils

$\beta$

Pfeilungswinkel des Tragflügels

$\varepsilon=L / D$

$\delta_{i j}$

$\Delta$

Gleitzahl, Verhältnis von Auftrieb zu Widerstand (auch $\frac{C_{l}}{C_{d}}$ )

Vertauschungsoperator

$\kappa=\frac{c_{p}}{c_{v}}$

Differenzoperator oder Laplacescher-Operator

Verhältnis der spezifischen Wärmen, $\left(c_{p}, c_{v}\right.$ hier: Wärmekapazitäten)

$\eta$

prozentuale Halbflügelspannweite

$\varphi$

Winkel

$\mathrm{V}$

kinematische Zähigkeit

$\rho$

Dichte

$\rho_{\infty}$

Dichte (Anströmung)

\section{Bezeichnungen aus den evolutionären Algorithmen}

$\mathrm{EM}$

Evolutionäre Methoden (gliedert sich in ES und GA)

ES

Evolutionäre Strategie

GA

Genetischer Algorithmus

SOGA

Singleobjective GA, Einfachoptimierung

MOGA

Multiobjective GA, Mehrfachoptimierung

\section{Sonstige Bezeichnungen}

OFW

Oblique Flying Wing 


\section{Einleitung}

Die Wirtschaftlichkeit moderner Transport- und Verkehrsflugzeuge steht im Mittelpunkt von wissenschaftlichem und kommerziellem Interesse. Diese stetig zu erhöhen und den aktuellen Erfordernissen anzupassen, ist vorrangige Aufgabe von Wissenschaft und Technik. Dabei läßt sich die Wirtschaftlichkeit von Verkehrsflugzeugen, strömungsmechanisch gesehen, in zwei wesentlichen Größen beschreiben. Diese sind der Auftrieb und der Widerstand. Das Ziel ist es, den maximalen Auftrieb bei minimalem Widerstand zu erlangen.

Die Bestrebungen, diese beiden Größen in ein optimales Verhältnis zu bringen, sind nicht neu. Der Auftrieb wird wesentlich durch die Tragflächen erzeugt. Der Widerstand ist der gesamte Widerstand von Rumpf, Tragflügeln und Leit- und Triebwerken. Jeder Versuch einer Verbesserung bezieht sich heutzutage nur auf einzelne Komponenten am Flugzeug. Man versucht die Tragflächen zu optimieren, oder am Rumpf den Widerstand zu reduzieren. Die grundsätzliche äußere Erscheinungsform eines Verkehrsflugzeuges selbst aber hat sich seit Jahrzehnten nicht verändert. Einzig die Pfeilung der Tragflächen hat dem erhöhten Widerstandsanstieg ungepfeilter Tragflächen im hohen Unterschallbereich Rechnung getragen. Für die Anforderung aus heutiger Sicht, die auch den Überschallbereich zu erschließen sucht, sind konventionelle Flugzeugauslegungen hochgradig ineffektiv. Die einzige konventionelle Auslegung dieser Art hat sich in der Concorde manifestiert. Diese Maschine fliegt weit jenseits jeder Wirtschaftlichkeit und wäre ohne besondere politische Unterstützung wirtschaftlich nicht zu halten.

Ein grundsätzliches Umdenken hat bis heute nicht stattgefunden. Willkommen sind nur Verbesserungen, die sich in das Konzept konservativer Flugzeugbauer nahtlos einfügen. Optimierungen sind nur in diesen engen Grenzen erwünscht, obwohl erhebliche Fortschritte erzielt werden könnten, wenn man das Konzept des Verkehrsflugzeuges als Ganzes überdenkt. Ansätze hierzu sind z.B. die Nurflügler, die in der ersten Hälfte des vorigen Jahrhunderts (Horten: 1943) schon Gegenstand der Forschung und Entwicklung waren. Die Entwürfe sind nach kurzen Erprobungsphasen verworfen worden, vorwiegend deswegen, weil die Steuer- und Regelungstechnik und die strömungsmechanischen Methoden selbst noch in den Kinderschuhen steckten.

Ein moderner Nachfolger dieser Nurflügler ist der OFW (Oblique Flying Wing). Dieser Entwurf stellt nach heutigem Erkenntnisstand das theoretisch zu erreichende Optimum hinsichtlich der Wirtschaftlichkeit im Überschall dar. Er ist in seiner Geometrie aber derart unkonventionell, daß mit einer Verwirklichung dieses Konzepts in naher Zukunft nicht zu rechnen ist.

Die Frage nach einem optimalen Verkehrsflugzeug ist auch die Frage nach der optimalen Geometrie. Für verschiedene Geschwindigkeitsbereiche sind verschiedene Geometrien optimal. Alle Geschwindigkeitsbereiche optimal abzudecken erfordert eine Änderung der Geometrie während des Fluges - diese Anforderung wird sicher auch mittelfristig nicht zu erfüllen sein. Geringe Fortschritte zur Geometrieänderung während des Fluges finden bisher nur in kleinem Rahmen statt. Durch Aufwölbung oder Abflachen des Profils (durch geeignete Mechanik im Tragflügel) läßt sich heute gerade erst die Lage des Verdichtungsstoßes (bei überkritischer Anströmung) geringfügig beeinflussen. Auch die Entwicklung der Steuerungselemente tritt mit der Schubvektorsteuerung und der Wirbelbeeinflussung auf den Tragflächen durch Canards (Vorflügel) in eine neue Phase.

Grundsätzlich ist die Wirtschaftlichkeit eines Verkehrsflugzeugs von der Anordnung seiner 
Komponenten und der Beschaffenheit der Oberfläche abhängig, d.h. es ist von der vorgegebenen Geometrie abhängig. In der Flugzeugaerodynamik ist eine der wichtigsten Aufgaben der Entwurf von optimalen Tragflächenformen. Welche Methoden stehen zur Verfügung, um diese Tragflächenformen für strömungstechnisch nichtlineare Problemstellungen zu erhalten?

Es ist offensichtlich, daß klassische gradientenbasierende Methoden diese Aufgabe nicht erfüllen können, weil diese Optimierungsmethoden die Eigenschaft haben, auf ihrer Suche nach einem globalen Optimum und nach Auffinden eines lokalen Optimums vorzeitig zu terminieren. Vielmehr ist eine Methode gefordert, die in einem mehrdimensionalen Parameterraum möglichst schnell und zuverlässig die Optima aufspürt. So eine Methode braucht nicht erfunden zu werden, sie existiert schon.

Nicht selten wird in Wissenschaft und Forschung die Natur selbst zu Rate gezogen, die für die komplexesten Probleme schon optimale Lösungen hat. Auch im weiten Feld der Aerodynamik gibt die Natur schon wesentliche Lösungen vor. Am Beispiel der Flügelgeometrie des Albatros wird deutlich, daß die Natur hier ein Optimum hinsichtlich Strukturstabilität, Auftrieb und Widerstand geschaffen hat. Das extrem hohe Seitenverhältnis des Flügels läßt den Albatros mühelos weite Strecken über Ozeane zurücklegen. Woher stammt also die optimale Flügelgeometrie des Albatros?

Es ist bekannt, daß optimale Anpassungen im Laufe der Evolution vollzogen werden. Die Evolution selbst stellt eine Methode dar, die Lebewesen nahezu perfekt an eine sich verändernde Umwelt anpaßt. Genau das ist auch das Ziel in Wissenschaft und Forschung. In diesem Fall sind es materielle Objekte, die unter vorgegebenen Randbedingungen (etwa ein Tragflügel in bestimmter Anströmung mit bestimmten Anstellwinkel, etc.) ein bestimmtes Ergebnis (ein maximales Verhältnis von Auftrieb zu Widerstand) erzielen sollen. Es scheint also zweckmäßig, nicht fertige Lösungen aus der Natur zu kopieren, sondern die Methode selbst zu kopieren, mit der die Natur über die Evolution zu optimaler Anpassung der Lebewesen gelangt ist. Diese Methode, implementiert als Algorithmus in einen Computer, kann dann auf Objekte angewendet werden, deren Optimierung für die Wissenschaft von Interesse sind. Diese Implementation ist verbunden mit der Hoffnung, Lösungen für Probleme zu finden, die in der Natur gar nicht vorkommen. Beispielsweise für aerodynamische Probleme im Überschall.

Mit dieser Idee vor Augen wurden vor etwa 25 Jahren die Evolutionären Methoden (im folgenden EM abgekürzt) entwickelt. Die genauen Bezeichnungen sind in der Literatur nicht festgelegt. Ich verwende hier die Evolutionären Methoden als Überbegriff für die Evolutionäre Strategie (ES) und die Genetischen Algorithmen (GA). Die Entwicklung der ES ist vor allem der Gruppe um Ingo Rechenberg [35] in Deutschland zuzuschreiben, die die Grundlage hierfür in den siebziger Jahren entwickelt hat. Etwas später entstehen in Amerika die Genetischen Algorithmen, deren erste Ansätze schon in den späten sechzigern Jahren zu sehen waren. John Holland kann wohl als einer der Väter der GA bezeichnet werden. Sein Werk [14] ist zwar schwer verständlich, enthält aber schon wesentliche Elemente des GA. Die Weiterentwicklung und theoretische Verfeinerung wird heute von der Gruppe um D.E. Goldberg [11] wesentlich beeinflußt.

Die beiden unterschiedlichen Methoden wuchsen in ihrer Bedeutung parallel zur Computerentwicklung. Erst durch moderne Parallelrechner und Großrechner findet sich ein weites Anwen- 
dungsspektrum. Die ersten Gehversuche für beide Strategien wurden aber zuerst experimentell durchgeführt [45]. Seit die Computer den heutigen hohen Leistungsstandard erreicht haben, liegt die Hauptanwendung der EM im theoretischen Bereich. Die Stärke der EM besteht darin, schnell einen hochdimensionalen Parameterraum parallel nach Lösungen abzusuchen. In der Praxis wird die Suche nach Erreichen einer adäquaten Lösung abgebrochen, die die gegebenen Anforderungen an die Lösung hinreichend befriedigt. Dabei zeigen sich die EM als ungeheuer robust und, mit geringen Einschränkungen, als äußerst „treffsicher“.

Allgemein kann man sagen, die EM gehören zu den gerichtet statistischen Methoden. Sie füllen damit das Spektrum am Ende einer Skala aus, an deren anderem Ende gradientenbasierte Methode vorherrschen. Die Methoden an den Enden der Skala sind sehr unterschiedlich; dennoch lassen sie sich miteinander zu den sogenannten hybriden Systemen kombinieren, wobei die übergeordnete Methode stets die EM ist, und z.B. lokal ein Simplexalgorithmus nachgeschaltet wird. Die hybriden Systeme bieten in wenigen speziellen Fällen Vorteile, können aber nicht grundsätzlich überzeugen.

Im Bereich der Optimierung und des aerodynamischen Entwurfs hat man früher auch sogenannte Expertensysteme verwendet. Eine damit verbundene Hoffnung war, daß man durch Anhäufung von Wissen (tatsächlich nur Information) ein Mittel zur Problemlösung in den Händen hält. Es zeigt sich schnell, daß eine reine Anhäufung von Information noch keinen wirklichen Nutzen bringt. Eine EM muß jedem datenbankorientierten, wissensbasierenden Expertensystem überlegen sein, weil Expertensysteme grundsätzlich nur Informationen, aber kein eigentliches Wissen speichern können. Man versuchte dem abzuhelfen, indem man den Informationen Regeln zur Anwendung zur Seite stellt. Natürlich hat so ein System Nutzen, aber immer sind auch sofort die Grenzen sichtbar, wenn es um die Auswertung der Information geht. Wissensbasierte Expertensysteme enthalten keinen implizierten Pfad, der einem zum Optimum führt. Sie geben bestenfalls Regeln zur Vermeidung von Fehlern an. Genau das ist ein wesentlicher Unterschied zu den EM. Die EM werten, parallel zu ihrer Anwendung, die Information sofort aus. So sind sie in der Lage, einen Weg zu einem globalen Optimum zu finden. Das ist ihre grundsätzliche Stärke, die sie für nichtlineare Problemlösungen so attraktiv macht.

Der Eindruck jedoch, der hier entstehen mag, daß man prinzipiell nur die EM implementieren muß und dann garantiert ein nahezu optimales Ergebnis erhält, täuscht. Die EM findet eine annähernd optimale Lösung nicht nur in den vorgegebenen Grenzen des Parameterraums sondern aufgrund des Mutationsprinzip auch außerhalb dieser Grenzen. Diesen Parameterraum kann man weit ausdehnen, aber um eine Lösung zu erhalten und zu überprüfen, braucht auch die EM Zeit. Deswegen sollte der abzusuchende Parameterraum klein sein, und durch wesentliche theoretische Erkenntnisse über das zu lösende Problem schon eingegrenzt sein.

Ein Hauptanwendungsgebiet der EM neben der Optimierung, insbesondere der GA, war ursprünglich das Gebiet der Künstlichen Intelligenz und der Machine Learning Systems. Hierzu gehören auch Gebiete wie Pattern Recognition. Man benutzt diese Methode, um z.B. Neuronale Netze zu trainieren. In diesem Bereich existieren keine anderen Methoden außerhalb der EM. Hier sind sie konkurrenzlos.

Eine Analyse der Problematik in der Aerodynamik und die Lösungsmöglichkeiten, die die EM anbieten, scheinen aufeinander abgestimmt zu sein. Im Rahmen der Auslegung eines Verkehrsflugzeuges sind aufeinander optimal abgestimmte Komponenten gefragt. Die Anzahl dieser 
Komponenten ist zwar nach oben weit offen aber endlich. Neben den Komponenten, die durch die Strömungsmechanik optimiert werden können, sind sehr viele weiteren Größen aus rein betriebswirtschaftlicher Sicht sauber aufeinander abzustimmen. Z.B. die Anzahl der Passagiere, die Flughöhe, die Flugzeit und die Reichweite. Diese Größen stehen natürlich in Wechselwirkung mit den technischen Randbedingungen - müssen also bei der Neukonzipierung eines Überschallverkehrflugzeugs von Anfang an miteinbezogen werden. Gerade zu den betriebswirtschaftlichen Aspekten ist die Arbeit von Van der Velden [8] ein interessanter Beitrag. Im Rahmen dieser Arbeit werde ich mich jedoch auf strömungsmechanische Aspekte, besonders auf die des geometrischen Entwurfs, beschränken. 


\section{Motivation und Zielsetzung}

Bei dem Entwurf aerodynamischer Konfigurationen (Profile oder Tragflügel) ist es nicht möglich, unbegrenzt Auftrieb erzeugen, ohne auch gleichzeitig großen Widerstand zu erzeugen. Ganz im Gegenteil erreicht man in einem ersten Entwurf stets einen Bereich großen Widerstandes mit kleinem oder mäßigen Auftrieb. Grundsätzlich gibt es also zwei Bereiche in einem Auftriebs-Widerstandsdiagramm die für einen aerodynamischen Entwurf kennzeichnend sind. Der Bereich hohen Widerstands und geringen Auftriebs ist leicht auszufüllen. Der Bereich hohen Auftriebs und geringen Widerstands ist dagegen wesentlich schwerer zu erreichen.

Diese schlichte Tatsache impliziert eine Grenze zwischen diesen beiden Bereichen. Kann man einen Bereich leicht ausfüllen und einen angrenzenden Bereich nicht erreichen, impliziert das eine Grenze für die Gleitzahl (Auftrieb/Widerstand). Diese Grenze würde dann das theoretische Maximum der möglichen Gleitzahl für verschiedene Widerstandswerte darstellen. Es stellt sich die Frage ob, diese Grenze überhaupt existiert. Und wenn sie existiert, dann ist die Methode von Interesse, mit der man diese Grenze erreicht, und wovon sie im Falle ihrer Existenz allgemein abhängt.

Innerhalb der EM gibt es die sogenannte Pareto-Optimierung. Diese Art der Optimierung gestattet es, eine Problemstellung nach zwei Größen gleichzeitig optimal zu gestalten, ohne die jeweils andere Größe zu verschlechtern. Für die hier aufgeworfene Problemstellung in der Aerodynamik könnte man den Auftrieb und den Widerstand (oder deren Beiwerte) als diese GröBen identifizieren. Diese Größen sind aufgrund ihrer enormen Bedeutung für die Wirtschaftlichkeit eines Verkehrsflugzeugs von besonderem Interesse.

Es ist offensichtlich, daß die Definition der Geometrie einer aerodynamischen Konfiguration bei dieser Vorgehensweise eine entscheidende Rolle spielt. Letztendlich ist es die Flexibilität der Geometrie selbst, die über eine optimale Anpassung an die Strömungsverhältnisse, und damit über die Güte der Adaption entscheidet. Das erfordert eine grundlegend gute Geometriedefinition. Eine analytische Definition von Oberflächen und allgemeinen Formen lassen sich mit dem Geometriegenerator von Sobieczky [40] realisieren. Insbesondere die vollständige analytische Definition durch geeignete Funktionen auf abgeschlossenen Intervallen kommt den hier gestellten Anforderungen sehr entgegen. Zusätzlich muß sich die Geometriedefinition mit den oben angestrebten evolutionären Verfahren verknüpfen lassen.

Der Einsatz von evolutionären Methoden in der Entwurfsaerodynamik ist nicht neu. Es existiert ein breites Feld von Anwendungen, wobei die beiden existierenden Verfahren der speziellen Problemstellung jeweils angepaßt wurden. Die meisten Anwendungen beziehen sich exakt auf einen Designpunkt (für Machzahl und Auftrieb), ohne die prinzipiellen Möglichkeiten einer Geometriedefinition auszuschöpfen. Die evolutionären Methoden werden auch für einen inversen Entwurf genutzt. D.h. man schreibt eine Druckverteilung vor, und versucht im Nachhinein die Profilkontur für diese Verteilung zu finden. Dabei bleibt meist unberücksichtigt, ob die Geometriedefinition die angestrebte Profilkontur überhaupt enthält. Insgesamt werde ich hier den umgekehrten Weg gehen, ich wähle eine Geometriedefinition, und prüfe welche maximalen Werte für die Gleitzahl erreichbar sind. 
Auf der Grundlage der evolutionären Methoden und der Basis einer ausgewählten Geometriedefinition soll ein Verfahren entwickelt werden, welches es erlaubt, unter Ausnutzung aller Möglichkeiten der Geometrievariationen an die oben genannte Grenze zu stoßen. Das Erreichen dieser Grenze würde einerseits Rückschlüsse darauf zulassen, wie die Geometrie zu gestalten ist, um zu einer widerstandsarmen Konfiguration (bei gleichzeitiger Maximierung des Auftriebs) zu gelangen. Andererseits würde diese Grenze ein Maß für die Güte der Geometriedefinition selbst darstellen. 


\section{Die Evolutionären Methoden}

\subsection{Motivation zur Nutzung Evolutionärer Methoden}

In der Aerodynamik existiert das Bedürfnis jede Komponente eines Flugkörpers optimal zu gestalten. Der Weg zu der optimalen Gestaltung einer Komponente hängt meist von der Aufgabe ab, die der Komponente als Teil eines gesamten Flugkörpers zukommt. Einzelne Komponenten $\mathrm{zu}$ optimieren ist im Zeitalter der fortgeschrittenen Computertechnik meist unproblematisch. Um z.B. einen vollständigen Tragflügel für ein Transport- oder Verkehrsflugzeug zu entwerfen, fahndet man in der Regel zunächst nach geeigneten Profilen für den Tragflügel, die aus einem zweidimensionalen Entwurfsverfahren resultieren. Diese Profile werden dann in die Planform des Tragflügels durch entsprechende Skalierung eingepaßt. Der logische Schluß an dieser Stelle ist dann, daß aufgrund der guten Profilierung auch der Tragflügel einen brauchbaren Entwurf darstellt. Hierbei sieht man zunächst grob von Einflüssen dreidimensionaler Strömungseffekte ab. Später zeigt sich dann immer, daß an vielen Stellen des Tragflügels tatsächlich auch die Profilierung nachgebessert werden muß, weil durch diese dreidimensionalen Strömungseffekte die anfänglich zweidimensionale Profilierung sich als nicht mehr ausreichend erweist.

Das so geschilderte Problem entsteht nicht nur in diesem Rahmen. In den meisten Fällen gestaltet man jede Komponente für sich optimal. Im Zusammenspiel mit anderen Komponenten ergeben sich dann immer Einschränkungen in der Leistungsfähigkeit des gesamten Flugkörpers. Diese Vorgehensweise ist strikt linear. Aus dem Schluß, daß jede Komponente für sich optimal entworfen und angepaßt ist, schließt man dann darauf, daß auch das Zusammenspiel aller Komponenten optimal sein muß. Was bei dem Entwurf von Beginn an vernachlässigt wurde, ist die Wechselwirkung, die die einzelnen Komponenten später im Rahmen eines vollständigen Flugkörpers untereinander ausüben. Diese Wechselwirkung ist es, die die anfänglich so optimal gestalteten Komponenten später im Zusammenwirken als weniger günstig erscheinen lassen.

Hieraus folgt sofort, daß das Zusammenwirken der Komponenten schon im ersten Entwurf berücksichtigt werden muß. Jede Komponente kann nicht mehr für sich allein entworfen werden. Vielmehr muß dem späteren Einfluß des Zusammenwirkens mit anderen Teilen in einer frühen Entwurfsphase Rechnung getragen werden. Das führt meist in einem frühen Stadium des aerodynamischen Entwurfs zu einer unangenehmen Häufung von Parametern. Damit sind dann die meisten Entwurfsverfahren überfordert. Das Ziel, eine Konfiguration als Ganzes optimal zu entwerfen, anstatt als Summe seiner optimalen Komponenten, scheitert, weil die Anzahl zu berücksichtigender Parameter schnell ansteigt. An dieser Stelle wird sichtbar, daß ein Entwurfsverfahren nicht an einer hohen Anzahl der Parameter scheitern darf, wenn der Flugkörper als Ganzes optimal gestaltet werden soll. Eine Optimierungsmethode muß in der Lage sein, aus einer großen Anzahl Parameter ein globales Optimum aus der anfänglichen Problemstellung zu finden. Die hohe Anzahl der Parameter läßt sich zumindest in der Aerodynamik grundsätzlich nicht vermeiden.

Es ist also zwingend erforderlich für ein aerodynamisches Entwurfsverfahren eine Methode zu finden, die einfach zu implementieren ist, die robust und flexibel einsetzbar ist und die möglichst schnell noch in großen Parameterräumen ein globales Optimum bestimmen kann. 
Wie so oft hält die Natur selbst Lösungen parat, die man sich in Wissenschaft und Technik zu eigen macht. Viele von der Natur geschaffene Lösungen sind für technische Anwendungen sehr attraktiv. Man denke dabei beispielsweise an das Verhältnis von Eigengewicht zu Strukturfestigkeit bei Spinnweben. Die Natur hat hier etwas geschaffen, was von Wissenschaft und Technik noch nicht kopiert werden kann. Die Erbauer von Hängebrücken sind bis heute auf ihre teuren und schweren Stahlseilkonstruktionen angewiesen. Von dem optimalen Verhältnis von Eigengewicht zu Strukturfestigkeit bei Spinnweben sind sie weit entfernt.

Auch für die oben erwähnte Problematik des aerodynamischen Entwurfs hat die Natur schon eine Lösung parat. Anders als sonst kann man hier jedoch keine fertige Lösung übernehmen. Die aerodynamischen Probleme liegen meist in einem anderen Umfeld, als sie für die Natur gegeben sind. Was jedoch aus der Natur kopiert werden kann, ist die Methode, mit der die Natur eine optimale Anpassung ihrer Lebewesen vollzieht. Diese Methode ist die Evolution. Die Evolution ist die treibende Kraft für die optimale Anpassung aller Lebewesen an ihren Lebensraum.

\subsection{Eigenschaften der Evolutionären Methoden}

Ein Blick auf die Natur und das hohe Maß an Anpassung der Lebewesen an ihre Umgebung, gibt einen Eindruck des Wirkens der Evolution. Jedes Lebewesen ist nahezu perfekt an seine Umwelt angepaßt und dort hochgradig überlebensfähig. Über einen langen Zeitraum hinweg hat die Evolution verschiedene Lebewesen hervorgebracht und diese an die verschiedenen Umweltbedingungen angepaßt. Selbst gravierende Umwälzungen der Lebensräume konnten die Entwicklung der Lebewesen nicht stoppen. Stets war die Evolution in der Lage, flexibel auf sich verändernde Randbedingungen zu reagieren. War ein Lebensraum zerstört, brachte die Evolution eine Lebensform hervor, die in der neuen Umgebung überlebensfähig war.

Im folgenden werden wir sehen, daß die Eigenschaften der Evolution vornehmlich durch die Genetik und deren Regeln zur Vererbung bedingt sind. Die Codierung der Erbmasse und die genetische Rekombination und Mutation im Wechselspiel mit der Selektion bilden die Substanz der Evolution. Die grundlegenden Prinzipien der Genetik werden im folgenden noch deutlich.

Der Grund für die Verwendung den evolutionären Methoden ergibt sich aus der Robustheit und Zuverlässigkeit dieser Strategien. Zwar sind diese äußerst rechenintensiv, aber letztendlich der einzige Weg, bei nichtlinearen Problemstellungen eine adäquate Lösung zu finden. Die deterministischen Optimierungsmethoden sind hier überfordert und können bestenfalls innerhalb der genetischen Algorithmen (z.B. Hybridisierung) einen Beitrag liefern.

An dieser Stelle können wir aber die wesentlichen Eigenschaften der evolutionären Methoden schon bestimmen. Die evolutionären Methoden

- beruhen auf einfachen Prinzipien (Rekombination, Mutation, Selektion),

- sind in der Lage, flexibel auf unterschiedlichen Randbedingungen zu reagieren,

- sind robust und „treffsicher“" (wenn es kein stark isoliertes Optimum gibt),

- sind in der Lage, hochdimensionale Parameterräume schnell und effizient auf ein globales 
Optimum hin zu untersuchen.

Gerade der letzte Punkt ist hier von großer Bedeutung. Eine grobe Abschätzung der Kombinationsmöglichkeiten der Nukleotidbasen (diese enthalten beim Menschen die genetischen Informationen) liefert $4^{3.000 .000 .000}$ Möglichkeiten. Das gibt einen Eindruck, aus welcher gigantischen Zahl von Möglichkeiten die Evolution die ,überlebensfähigen“ Lebewesen herausfindet.

Die hier aufgeführten Eigenschaften sind exakt die, die man für den Entwurf einer brauchbaren aerodynamischen Konfiguration benötigt. Insbesondere verspricht eine Implementation der Evolution in einen Computeralgorithmus die Problematik der hohen Anzahl von Parametern zu lösen.

\subsection{Der Genetische Algorithmus}

Grundsätzlich läßt sich zwischen zwei unterschiedlichen Methoden im Rahmen der evolutionären Methoden unterscheiden. In der Literatur ist diese Gliederung nicht namentlich festgelegt. Ich verwende hier den Begriff Evolutionäre Methoden (im folgenden EM abgekürzt) als Überbegriff für die Evolutionären Strategien (im folgenden ES abgekürzt) und genetischen Algorithmen (im folgenden GA abgekürzt).

Die Entwicklung der ES ist vor allem der Gruppe um Ingo Rechenberg [35] in Deutschland zuzuschreiben, die die Grundlage hierfür in den siebziger Jahren entwickelt hat. Etwas später entstehen in Amerika die Genetischen Algorithmen, deren erste Ansätze schon in den späten sechzigern Jahren zu sehen waren. John Holland [14] und D.E. Goldberg [11] können wohl als Väter der GA bezeichnet werden. Beide Methoden wuchsen in ihrer Bedeutung parallel zur Computerentwicklung. Die ersten Gehversuche für beide Strategien wurden zuerst experimentell durchgeführt.

Jede der beiden evolutionären Methoden hat ihre Vorzüge. Bei den Evolutionsstrategien stehen dabei die Mutationsprozesse und die adaptive Schrittweitenregelung im Vordergrund, bei den genetischen Algorithmen hingegen eher die genetische Rekombination und die Crossover-Mechanismen. Keine der beiden Methoden genießt zur Optimierung einen prinzipiellen Vorzug. Die Auswahl der Methode zur Lösung eines konkreten Problems ist extrem vom Problem selbst abhängig. Grob kann man sagen, daß die GA Vorteile bei großen Suchräumen haben, während die ES in kleinen Suchräumen ihre Stärken entwickeln. Dieses ist eine sehr allgemeine Aussage, die keinen Beitrag zur Lösung eines konkreten Problems liefert. Anscheinend werden aber im Allgemeinen die GA bevorzugt und scheinen bei der Mehrfachoptimierung in ihrer Funktionalität besser entwickelt zu sein.

Im einem ersten Schritt kann eine Übersicht über die genetischen Algorithmen in [2], [3] und [11] gewonnen werden. Die Grundlagen und wichtigsten Mechanismen der Evolutionsstrategien werden in [35] und [38] entwickelt. In [37] werden diese den GA gegenübergestellt. Hier werden auch die Fundamente der Evolutionsstrategien und der genetischen Algorithmen zur Optimierung in einer guten Übersicht wiedergegeben. Speziellere Anwendungen in Forschung und Technik werden in [24] behandelt. 
Die Idee zur Lösung schwieriger technischer Probleme in Form eines genetischen Algorithmus ist aus dem biologischen Umfeld entnommen. Die Beobachtung, daß viele Lebewesen heute an ihre Umgebung optimal angepaßt sind und daß die Lebewesen selbst eine Entwicklung ihres äuBeren Erscheinungsbildes durchgemacht haben, legt die Annahme einer quasi zielgerichteten Entwicklung (Optimierung) ihrer Erscheinungsform nahe, die man auch für physikalisch-technische Probleme zu nutzen sucht.

Die Entwicklung der Erscheinungsformen an sich ist nicht a priori zielgerichtet, sondern die Entwicklung wird durch den sogenannten Selektionsdruck gelenkt. Die Lebewesen sind in ihrem Dasein einem Auswahlkriterium unterworfen, welches über Weiterbestehen oder Aussortieren entscheidet. Sind die Lebewesen gut an ihre Lebensräume angepaßt, steigt die Wahrscheinlichkeit, daß sie überleben. Es findet eine Auswahl (Selektion) statt, die über diesen Umweg Einfluß auf das genetische Material nimmt (Selektionsdruck). „Gene können nicht sehen“, daher kann ein Gen nicht „wissen“, ob das hervorgebrachte Lebewesen gut oder schlecht ist. Die Rückmeldung, ob Gene ein gutes (überlebensfähiges) oder ein schlechtes (nicht überlebensfähiges) Lebewesen hervorgebracht haben, geschieht in abstrakter Form durch die Überlebensrate. Ist diese Rate hoch, so handelt es sich um gute Erbanlagen, die dieses Lebewesen hervorgebracht haben. Das Lebewesen ist überlebensfähig und reicht einen wesentlichen Teil der Erbanlagen an die nächste Generation weiter. Sterben bestimmte Lebewesen aus, so stirbt die „schlechte“ Erbanlage mit. Auf diese Art und Weise erfährt die Entwicklung der Lebewesen eine Richtung hin zur optimalen Anpassung an ihre Umgebung. Je besser ein Lebewesen angepaßt ist, je häufiger wird diese Art überleben, und um so wahrscheinlicher ist die Verbreitung seiner Erbanlagen.

Diejenigen Lebewesen, die die Selektion (Auswahl der Besten) überstanden haben, bilden Nachkommen und kombinieren ihr Erbgut untereinander neu (Rekombination). Bei der Weitergabe der Erbanlagen können unter Umständen Fehler (Mutationen) auftreten. Die Wahrscheinlichkeit, daß Mutationen auftreten, ist in der Regel gering. Aus dem vorhandenem genetischen Material der guten Erbanlagen werden neue Kombinationen von Erbanlagen gebildet. Diese neue Generation wird wieder auf ihre Widerstandfähigkeit gegenüber Umweltbedingungen getestet und nur die besten Individuen der Generation für die Nachkommenschaft ausgewählt. So findet eine stetige Verbesserung der Anpassung an die Umgebung statt. Man könnte sagen, daß die Gesamtheit aller Lebewesen gegen ein bestimmtes, optimal angepaßtes Lebewesen konvergieren. Der Phänotypus (s.u.) der Lebewesen innerhalb einer Art ist dann ungefähr für alle derselbe. Schematisch läßt sich ein GA wie in Abb. 1 beschreiben.

An dieser Stelle mache man sich klar, daß die Lebewesen im Grunde zwei Erscheinungsformen haben. Diese sind der Genotypus und der Phänotypus. Der Genotypus ist die Erscheinungsform als ,,abstrakter Parameterstrang“. Beim Menschen bezeichnet man das als die DNA. In der hier angestrebten Arbeit, der Geometriedefinition, kann der Genotypus ein eindimensionaler Vektor reeller Zahlen sein. Der Phänotypus ist die letztendliche Erscheinungsform, das, was quasi aus dem Genotypus erwächst. So wie die DNA die grundsätzliche Codierung eines Menschen ist, wird hier der eindimensionale Vektor reeller Zahlen die grundlegende geometrische Definition eines strömungsmechanischen Objekts sein (z.B. eines Profils oder Tragflügels).

Wie oben schon erwähnt, unterliegen Genotypus und Phänotypus einer Wechselwirkung. Aus dem Genotypus bildet sich nach einer nicht näher definierten Abbildungsvorschrift der Phäno- 
typus. Die genaue Abbildungsvorschrift (also die Entwicklung von der DNA zum vollentwikkeltem Menschen) ist auch beim Menschen nicht exakt erforscht. Deswegen kann man davon ausgehen, daß diese Abbildungsvorschrift beliebig abstrakt sein darf. Die umgekehrte Wirkung vom Phänotypus auf den Genotypus geschieht, wie schon oben erwähnt, über die Selektion. Denn sollte sich der Phänotypus, der den Umweltbedingungen ausgesetzt ist, sich nicht bewähren und nicht weiterexistieren, so verschwindet auch sein genetisches Material. Somit wird aus dem Topf vorhandenen genetischen Materials stets nicht brauchbares Material entnommen. Das führt zu einer Verbesserung des genetischen Materials und damit auch zur Verbesserung des Phänotypus (also des Profils oder Tragflügels).

\section{Der Genetische Algorithmus als PseudoCode}

Codierung: wähle eine geeignete Codierung der Erbinformationen,

Initialisierung: gestalte die Individuen einer Population nach dem

Zufallsprinzip. Das ist die Generation mit der Nummer 1.

\section{LOOP:}

Evaluation: Bewerte die Individuen nach einer Fitneßfunktion (Ranking),

Abbruchkontrolle: ist eine zufriedenstellende Lösung gefunden?

Selektion: wähle Paare von Individuen für ein „Heiratsschema“ aus,

Rekombination: Bildung von Nachkommen der aktuellen Generation,

Mutation: Die Nachkommen werden mit einer geringen Wahrscheinlichkeit mutiert,

Ersetze die Individuen: gemäß Ersetzungsschema (Survival of the fittest) werden gering bewertete Individuen durch die Nachkommen in der Population ersetzt.

GOTO LOOP

\section{Abb. 1 Der Genetische Algorithmus als PseudoCode.}

Dieses ist die grundlegende Beschreibung eines genetischen Algorithmus. Hinter dieser einfache Fassade verbergen sich viele Feinheiten, die für verschiedene Arten von Optimierungen wichtig sind.

\subsubsection{Codierung}

Der erste Schritt des Algorithmus ist die Wahl einer geeigneten Codierung. Dieser Schritt bezieht sich, wie im folgenden noch ausgeführt werden wird, auf die Implementation einer Geometriedefinition (für ein Profil oder Tragflügel) in eine genetische Codierung. Das genaue Vorgehen wird weiter unten behandelt werden. Hier sei nur einmal darauf hingewiesen, daß die 
Geometriedefinition so zu wählen ist, daß jeder Parameter denselben Einfluß auf den Phänotypus hat. Denn hat ein Parameter keinen oder nur geringen Einfluß auf das Ergebnis, so könnte man ihn auch weglassen, und damit den abzusuchenden Parameterraum verkleinern. Ebenso sind sogenannte epistatische Effekte zu vermeiden. Das Wesen der Epistasis ist an einem Beispiel gut zu verstehen. Nimmt man im Falle des Menschen an, daß ein Gen über Haarwuchs oder Kahlheit entscheidet, und die Haarfarbe über ein anderes Gen bestimmt ist, so hat das Gen der Haarfarbe keinen Effekt mehr, wenn das andere Gen schon die Kahlheit vorgegeben hat. In der Geometriedefinition für strömungsmechanische Objekte kann man erst im Nachhinein eventuell auftretende Epistasis feststellen.

\section{Binäre Codierung}

Üblicherweise ist die Codierung bei einem GA binär. D.h. der Genotypus besteht aus einem Vektor, dem als Phänotypus eine bestimmte Bedeutung zukommt. Die genetische Codierung zweier Objekte kann ganz allgemein wie folgt aussehen:

$$
\begin{aligned}
& \text { Gencode }\left(\text { Objekt }_{A}\right)=\{1,0,0,0,0,1,1,0\} \\
& \text { Gencode }\left(\text { Objekt }_{B}\right)=\{1,1,0,0,1,0,0,0\}
\end{aligned}
$$

Der genetische Code besteht aus einem Vektor der Länge n (hier n=8). Im Falle der Binärcodierung sind die Elemente des Vektors Nullen und Einsen. Viele theoretische Betrachtungen bevorzugen für die Entwicklung der Beweisführung die Binärdarstellung. So läßt sich auch eines der wichtigsten Theoreme der EA sehr übersichtlich aus der Binärcodierung entwickeln. Diese Darstellung ist allerdings für umfangreiche numerische Berechnungen ungeeignet.

\section{Reelle Codierung}

Im Falle der Binärcodierung bestehen die Elemente der Vektoren nur aus den Zahlen Null und Eins. Die Elemente des Vektors können jedoch auch beliebige Gestalt annehmen. Für die genetischen Operationen sind reelle Zahlen die geeigneten Elemente. Im Vorgriff auf die geometrische Definition sei hier schon zur Erklärung die genetische Darstellung eines Kurvenabschnitts vorweggenommen. In der reellen Codierung hat dann der genetische Satz dann die folgende Form:

$$
\text { Gencode(Kurvenabschnitt })=\left\{k e y, g, \varphi, r, s_{1}, e_{1}, s_{2}, e_{2}, \varphi_{1}, r_{1}\right\}
$$

Mit key-spezielle Kennzahl, $g$-Funktionskennzahl, $\varphi, r, \varphi_{1}, r_{l}$-Anfangs- und Endpunkt, und $s_{l}$, $e_{1}, s_{2}, e_{2}$-Parameter der Funktionen $g$. Über die spezielle Kennzahl key ist es möglich, die Zugehörigkeit des genetischen Satzes festzulegen. Weiterhin ist es dadurch möglich, eine Kurve aus mehreren Teilabschnitten zusammenzusetzen, wie es hier teilweise erforderlich sein wird. Die verschiedenen Teilstücke einer Kurve sind über die Kennzahl key zu identifizieren. Diese dient weitgehend zur Organisation der Geometriedefinition. 


\subsubsection{Initialisierung}

Man bestimmt eine geeignete Anzahl von Objekten, von der man annimmt, daß sie der Problemstellung angemessen ist, und nach einer begrenzten Zahl von Iterationen des GA eine adäquate Lösung hervorbringt. Diese Anzahl liegt von vornherein nicht fest. Sie ist problemspezifisch. Insbesondere bei der Mehrfachoptimierung erfordert die Bestimmung dieser Anzahl eine gewisse Sensibilität. Allgemein gilt, wenn die Anzahl von Objekten (die Individuen einer Population) groß ist, wird der GA ein befriedigendes Ergebnis liefern. Im Falle der Initialisierung eines eindimensionalen Vektors bedeutet eine Initialisierung eine zufällige Besetzung des Vektors mit reellen Zahlen. Diese reellen Zahlen sind je nach ihrer Bedeutung einer Grenze unterworfen.

\subsubsection{Evaluation}

Die Evaluation wird üblicherweise durch eine Zuweisung des sogenannten Fitneßwertes zu einem Objekt (Individuum) vorgenommen. Dieser Wert gibt an, wie gut sich der Phänotypus in der gegebenen Umwelt bewährt hat. Dieser Wert wird praktischerweise auf das Intervall $[0,1]$ skaliert. Im weiteren Verlauf des GA wird die Bedeutung dieses Wertes klar. Er hat Einfluß darauf, ob das Objekt (Individuum) zunächst überhaupt überlebt, und wenn ja, mit welcher Wahrscheinlichkeit sich das genetische Material dieses Objekts innerhalb der Population ausbreitet. Für die Mehrfachoptimierung wird das Prinzip des Rankings angewendet, welches weiter unten im Kapitel der Pareto-Optimierung ausführlich behandelt ist.

Nach der Evaluation wird stets getestet, ob ein befriedigendes Ergebnis erreicht ist und der Algorithmus gegebenenfalls abgebrochen werden kann.

\subsubsection{Selektion}

In diesem Abschnitt wird aufgrund des Fitneßwertes ausgewählt, welche Objekte in die nächste Generation übernommen werden und aus welchen Objekten die Nachkommenschaft gebildet wird. In dem hier verwendeten GA lasse ich die Anzahl der Objekte konstant. Es gibt GAs, die mit dynamischer Populationsgröße arbeiten. Das führt meist zu einer deutlichen Vergrößerung der Anzahl von Individuen und kann sich negativ auf die Konvergenzgeschwindigkeit auswirken. Eine dynamische Populationsgröße sollte man nur in ausgewählten Fällen in Betracht ziehen. Im Gegensatz zu den beiden folgenden Mechanismen der Rekombination und Mutation ist die Selektion der einzige Mechanismus, der einer Ausweitung des Phänotypus entgegenwirkt. Denn Rekombination und Mutation bilden stets neue Phänotypen und verbreitern sozusagen die Basis des Phänotypus. Die Selektion schränkt diese Basis wieder auf ein gewisses Maß ein.

\subsubsection{Rekombination}

Dieser Mechanismus ist einer der entscheidenden innerhalb des GA. Die Rekombination sorgt für eine Durchmischung der Population innerhalb vorgegebener Grenzen. Das entspricht einer weiträumigen Suche in einem n-dimensionalen Parameterraum. Die Nachkommenschaft wird aus zwei Elternteilen rekombiniert. Es gibt auch hier wiederum verschiedene Mechanismen, die sich hauptsächlich auf der Auswahl der Elternteile für die Nachkommenschaft bezieht. Z.B. kann man die Nachkommenschaft nur aus N-Besten Eltern auswählen, und den Elternteil mit mittleren Fitneßwerten vernachlässigen. Bei solchen Auswahlmechanismen ist stets Vorsicht 
geboten, da durch die eingeschränkte Auswahl der Eltern es eventuell zur vorzeitigen Konvergenz kommen kann (Premature Convergence).

Prinzipiell wird eine binäre Rekombination, wie in Abb. 2 sichtbar, durchgeführt. Hierfür werden nach dem oben genannten Verfahren zwei Objekte ausgewählt. In der binären Codierung wird eine Zahl, kleiner als die Länge des Vektors, bestimmt und Vektor dort aufgebrochen. Durch Austausch (Uniform Crossover) oder Mittelung der Elemente zweier Vektoren entsteht ein neuer Vektor. In der binären Codierung wird eine Zufallszahl i bestimmt, der Vektor an dieser Stelle aufgebrochen und an den verbliebenen Teil des zweiten Vektors angefügt. Ebenso werden die ersten Elemente des zweiten Vektors an den verbleibenden Elementen des ersten Vektors angefügt. Aus der Vertauschung der Endstücke des Vektor, wie in Abb. 2 sichtbar, entstehen zwei neue Vektoren, die den genetischen Codes repräsentieren, und damit auch zwei neue Objekte.

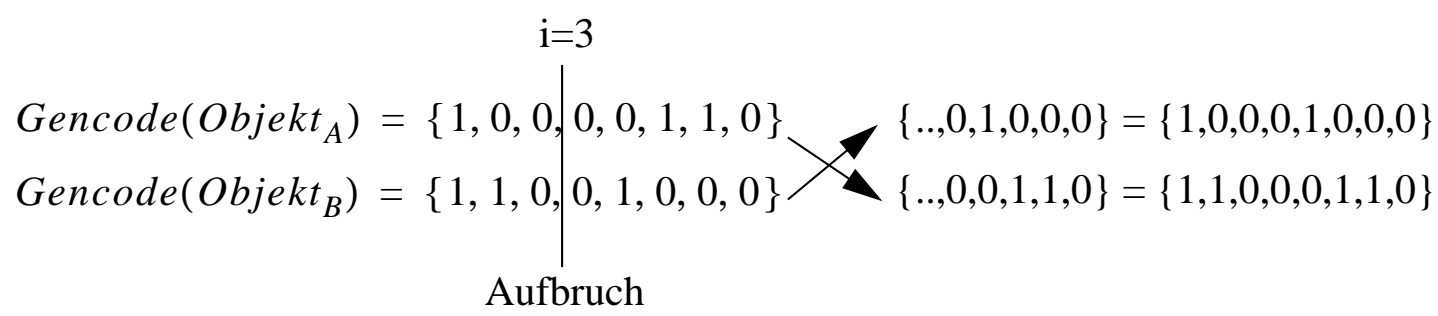

\section{Abb. 2 Binäre Rekombination.}

Die so vollzogene Rekombination wird als Uniform-Crossover bezeichnet, weil der genetische Code an exakt einer Stelle aufgebrochen wird. Es gibt hier Varianten, z.B. Two-Point-Crossover, dort wird der genetische Code an zwei Stellen aufgebrochen und die Mittelstücke der Objekte vertauscht. Es ist allerdings zur Zeit nicht erwiesen, daß der Aufbruch des genetischen Codes an mehreren Stellen einen sichtbaren Vorteil bringt.

In dieser Arbeit verwende ich die reelle Rekombination, die, wie man weiter unten sehen wird, eine erhebliche Vereinfachung in der Handhabung darstellt. Die binäre Rekombination sollte hier allerdings nicht unerwähnt bleiben, weil viele Theoreme der GA auf der binären Codierung basieren.

\section{Reelle Rekombination}

Im Unterschied zur binären Rekombination wird der genetische Code nicht an einer Stelle aufgebrochen, sondern die Elemente des Vektors über folgende Vorschrift rekombiniert.

Zur Rekombination werden wie üblich zwei Eltern-Objekte Objekt ${ }_{\mathrm{A}}$, Objekt $_{\mathrm{B}}$ herangezogen. Über eine Zufallszahl $r$ werden aus den zwei Elementen $a, b$ zwei neue Elemente $c, d$, wie in Abb. 3 sichtbar, bestimmt. Die neuen Elemente bilden dann die neuen Objekte ${ }_{C}$ und Objekt $_{D}$. 


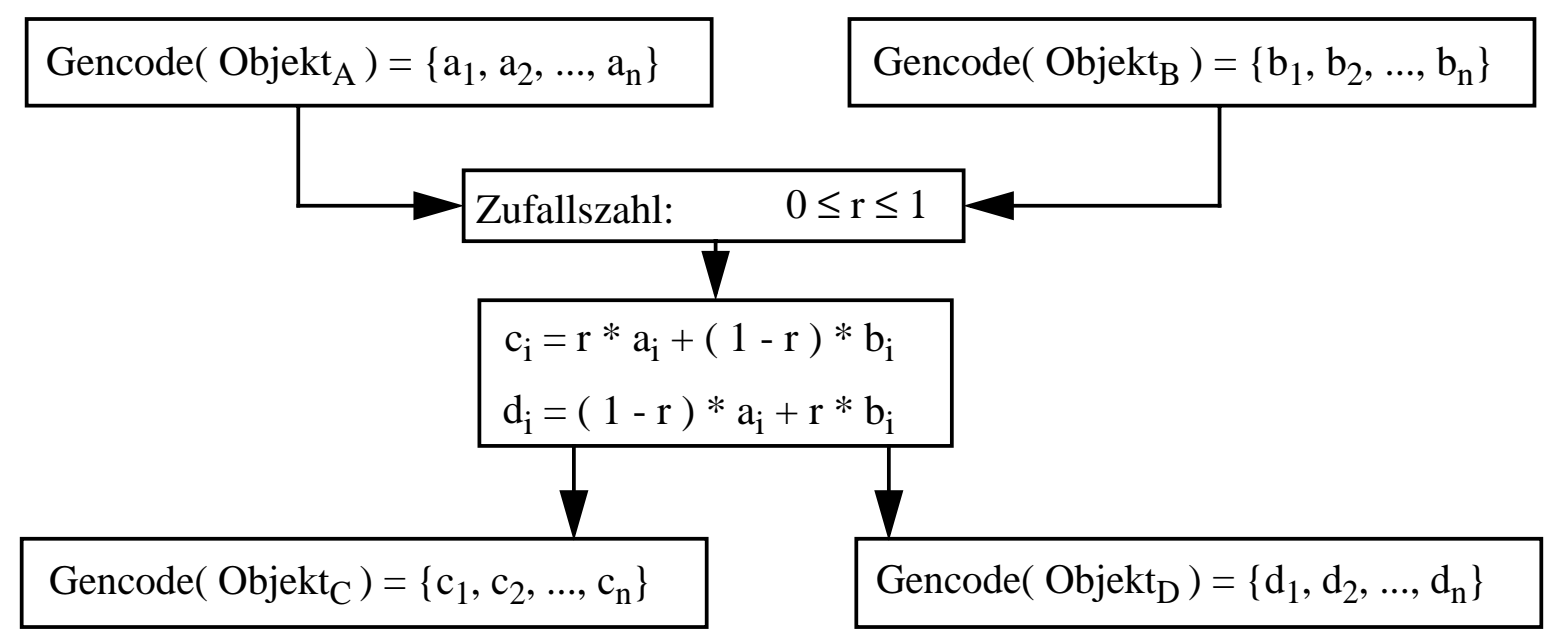

Abb. 3 Reelle Rekombination.

Diese einfache Rekombinationsvorschrift sucht sehr effektiv den Parameterraum zwischen den Elementen $a, b$ ab. Diese Suche ist weiträumig, d.h. das neu rekombinierte Element $c$ oder $d$ kann als Parameter jeden Wert zwischen $a$ und $b$ annehmen. Die Suche ist zwar schnell aber auf die Grenzen $a, b$ beschränkt. Denn durch die so durchgeführte Rekombination wird der Parameterraum die einmal vorgegebenen Grenzen bei der Initialisierung eines Parameters $(\min (a)<a$ $<\max (\mathrm{a}))$ niemals überschreiten. Für den Phänotypus bedeutet das, daß er auf ein bestimmtes Maß beschränkt ist, und keine Anpassung seiner Erscheinungsform außerhalb des anfangs vorgegebenen genetischen Codes realisieren kann. Erst die Mutation wird in der Lage sein, diese Grenzen zu überschreiten.

\subsubsection{Mutation}

Die Rekombination ist im Verhältnis zur Mutation eine grobe Methode, den n-dimensionalen Parameterraum nach geeigneten Lösungen abzusuchen. Durch Mutation werden mit geringer Wahrscheinlichkeit geringfügige Abweichungen von den originalen Vektoren erzeugt. Dadurch werden Gebiete nach Lösungen abgesucht, deren Abstand sehr klein ist zu den ursprünglichen Vektoren. Durch die Mutation werden dicht beinanderliegende Parameterkombinationen auf eine sinnvolle Lösung hin getestet und eventuell Lösungen gefunden, die außerhalb der vorgegebenen Parametergrenzen liegen. Das ist der wichtigste Unterschied zu der Rekombination. Das Auftreten von Mutationen darf nur mit geringer Wahrscheinlichkeit geschehen, da sonst die Wirkung der Rekombination beeinträchtigt wird.

$$
c_{\text {mut }}=c_{\text {rek }} \pm \varepsilon
$$

$\mathrm{Zu}$ dem Parameter $c_{r e k}$ in (3), der durch Rekombination neu entstanden ist, wird ein kleiner Wert, meist im Bereich von $\pm 1 \%$, addiert und bildet so den mutierten Parameter $c_{\text {mut }}$. Wenn $c_{r e k}$ schon selbst nahe dem minimalsten Wert für $c$ in der gesamten Population liegt, dann kann $c_{m u t}$ durchaus schon außerhalb der Grenze liegen, die bei der Initialisierung für $c$ vorgegeben war. 
Sollten sich die Phänotypen, deren Parameter außerhalb dieser Grenze liegen, bei der Selektion bewähren, so setzen sich die neuen genetischen Strukturen vermehrt durch. Die neue Grenze für einen bestimmten Parameter umschließt dann die Grenze des alten Parameterraums. Eine veränderte Erscheinungsform des Phänotypus wird dann realisiert, weil sich die genetische Basis verbreitert hat. Die genetische Basis liefert dann neue, veränderte Erscheinungsformen und lotet neue Grenzen aus.

\subsubsection{Ersetzung}

In einem letzten Schritt wird der Teil der Generation, deren Fitneßwerte im unteren Bereich der Skala liegen, durch die neugebildete Nachkommenschaft in der Population ersetzt. Der Ring schließt sich hier. Nach diesem Schritt wird wieder der Fitneßwert berechnet und die Population (in der Evaluation) neu bewertet.

Der Eindruck, der hier entsteht, daß im Grunde genommen nur eine geeignete Codierung für die Problemstellung gefunden werden muß, und dann durch Anwendung eines GA ein optimales Ergebnis erzielt wird, ist trügerisch. Tatsächlich findet ein GA ein sehr gutes Ergebnis, wenn das globale Optimum nicht extrem lokal begrenzt ist. Aber es gibt auch eine Reihe von Testfunktionen, [37], die die Leistungsgrenzen und Unterschiede der GA und ES beschreiben. Es soll daher hier nicht unerwähnt bleiben, daß der Erfolg der Anwendung eines GA nicht nur von den oben genannten Mechanismen abhängt, sondern ebenso von der Stärke des Einflusses, den man einem bestimmten Mechanismus zuordnen kann. Anhand der Mutation wird das deutlich. Als Mechanismus eines GA steht die Notwendigkeit der Mutation außer Frage, aber man kann ihren Einfluß erhöhen, indem man statt 1\% Mutationen etwa 10\% Mutationen zuläßt. Unter gewissen Umständen mag das sinnvoll sein. Den Mechanismen des GA sind also prozentuale Raten zugeordnet, die die Größe ihres Einflusses auf den GA repräsentieren. Für folgende Einflußgrößen sind zugeordnete prozentuale oder absolute Zahlen von Bedeutung:

- Population Size (Anzahl der Individuen),

- Crossover Rate,

- Mutation Rate,

- Generation Gap (Überlebensrate),

- Scaling Window,

- Selection Strategy.

Die englische Bezeichnung sei wegen der Konformität mit der Literatur hier gestattet.

Es gibt Ansätze, einen GA selbst zum Gegenstand eines GA zu machen, ein Beispiel hierzu findet man in [4]. Man spricht dann von einem Meta-GA, d.h. zur Optimierung der Parametereinstellung eines GA benutzt man einen GA. Prinzipiell findet man in einem weiten Parameterbereich des GA eine sehr gute Lösung. Der GA würde, wenn die GA-Parameter nicht hochgradig exotisch gewählt werden, in der Regel gegen eine optimale (oder suboptimale) Lösung konvergieren. Ist die Population Size zu klein, liegt aber möglicherweise keine optimale 
Lösung vor, weil die „Unterräume“ unzureichend durchsucht wurden. Eine Erhöhung der Populationsgröße kann dann eine verbesserte Lösung bringen. Insbesondere die Populationsgröße (Population Size) wird im folgenden noch bei der Mehrfachoptimierung eine wichtige Rolle spielen. Tatsächlich muß die Größe für jede Problemstellung extra bestimmt werden.

\subsection{Die Evolutionäre Strategie}

Innerhalb der evolutionären Methoden bildet diese Strategie eine Alternative zu dem GA. Die ES versucht eine Optimierung dadurch zu erreichen, daß die Parameter der darstellenden Funktion nach dem Zufallsprinzip geringfügig verändert werden. Die durch die darstellende Funktion beschriebenen Objekte werden dann durch den Fitneßwert bewertet und entsprechend sortiert. Im Gegensatz zum GA werden jetzt keine neuen Objekte durch Rekombination ,,überlebender Objekte" gebildet, sondern die neuen Objekte entstehen aus Mutationen der alten Objekte. Die Verbesserungen in der Gesamtheit entstehen hier durch mehrfache Wiederholung dieses Prozesses. Im Vergleich zum GA ist das Verfahren ähnlich, es fehlt im Vergleich hierzu praktisch nur die Rekombination und ist somit im Anfangsstadium der Optimierung sehr langsam. Möglicherweise kann aber genau diese Methode im Endstadium der Berechnung eine Hilfe sein sich den theoretisch möglichen Werten noch weiter anzunähren. Das Ergebnis, das der GA liefert, ist, wie oben schon erwähnt, nicht perfekt, aber dem optimalen Wert sehr nahe. Um diese kleine Differenz zwischen theoretischem und durch den GA erhaltenen numerischen Wert noch weiter zu verkleinern, könnte die evolutionäre Strategie von Nutzen sein.

Wie oben schon erwähnt sind GA und ES Methoden verschiedener Schulen. Die GA wurden vorzugsweise in den USA, die ES hauptsächlich in Deutschland entwickelt. Die Evolutionsstrategen verfolgten von vornherein einen an reellen Zahlen orientierten Codierungsansatz. Von Beginn an wurden die Individuen durch Vektoren reeller Zahlen codiert, und eine Population von Individuen war einfach eine Menge solcher Vektoren. Die einfachste Form der ES zeichnet sich durch drei Schritte aus.

- Selbstreproduktion,

- Mutation,

- Selektion.

Es ist auffällig, daß hier der Schritt der Rekombination fehlt. Dieser wurde in der späteren Entwicklung der ES hinzugefügt. Trotz quasi gleicher evolutionärer Prinzipien unterscheiden sich die ES von den GA.

Die (1+1)-ES ist die einfachste Form dieser Strategie. Sie wurde 1964 von Rechenberg in einen seiner klassischen Experimente zur optimalen Einstellung von Gelenkwinkeln einer Gelenkplatte eingesetzt. Ausgehend von einem „Ur-Individuum“ (einem Vektor reeller Zahlen) wird ein zweites Individuum (ein zweiter Vektor) als Nachkomme durch einfaches Duplizieren des Ausgangsvektors erzeugt. In einem zweiten Schritt wird das Duplikat zufällig, aber nicht willkürlich, modifiziert. Das Duplikat wird mutiert, indem auf jeden einzelnen Parameter des Vektors ein zufälliger (in der Regel kleiner) positiver oder negativer Wert addiert wird. Im nächsten Schritt werden beide Individuen bewertet. Demjenigen, dem die Fitneßfunktion den höheren Wert zuordnet, überlebt. Der andere wird nicht weiter beachtet. Nach diesem Schema wird wei- 
ter verfahren, bis ein hinreichender Fitneßwert eines Individuums erreicht wird.

Der serielle Charakter der (1+1)-ES (1 Elter, 1 Nachkomme) wird logisch durch die $(\mu+\lambda)$-ES ( $\mu$-Eltern, $\lambda$-Nachkommen, mit $\lambda \geq \mu \geq 1$ ) erweitert. $\mu$ und $\lambda$ repräsentieren hier ganze Zahlen. Das $\mu$ gibt hier die Anzahl der Eltern an. Das $\lambda$ gibt die Anzahl der Nachkommen an. Die Evolutionsschritte sind im wesentlichen wie bei der (1+1)-ES. Die Auswahl trifft mit gleicher Wahrscheinlichkeit jedes Individuum der Elternpopulation. Aus den $\mu$ Eltern werden $\lambda$ Nachkommen produziert. Aus den $\mu+\lambda$ - Individuen werden nur die besten $\mu$ ausgewählt. Die Anzahl der Eltern bleibt damit konstant.

Das so durchgeführte $(\mu+\lambda)$ Auswahlverfahren hat einen wichtigen Effekt: die Qualität der Besten wird von Generation zu Generation nie schlechter. In der $(\mu, \lambda)$-ES hingegen werden nur noch die $\mu$-Besten aus den $\lambda$-Nachkommen selektiert. Die Qualitätsfunktion kann hier dann starken Schwankungen unterlegen sein.

Die bisher erwähnten ES machen keinen Gebrauch von der Rekombination. Um dieses Manko zu beseitigen, schuf man die $(\mu / \rho \# \lambda)$-ES. Der wesentliche Unterschied besteht in der Erzeugung der Duplikate. Dazu werden Gruppen von $\rho$ Elementen gebildet. Im Fall $(\rho=2)$ werden $\lambda$-Zweiergruppen gebildet. Um wieder $\lambda$-Nachkommen zu erhalten, gibt es hier für die Rekombination zwei verschiedene Arten. Aus den Gruppen werden jeweils über jede Vektorkomponente Mittelwerte und Streuung berechnet. Die Elemente des Vektors eines Nachkommens ist aus den Mittelwerten dieser $\rho$-Gruppen gebildet, enthält dann also eine Erbinformation aller Elternteile im gleichen Maße. Die zweite Methode ist, jeweils zufällig jedes Element des Elternvektors in den Nachkommenvektor einzusetzen (Uniform Crossover).

Ein weiterer Schritt ist, noch Subpopulationen einzuführen, die sich getrennt vom Rest der Gesamtpopulation weiterentwickeln können. Die hier vorgestellten ES lassen sich kombinieren und schachteln. Die Erfahrung lehrt aber, daß nach der zweiten Schachtelungsstufe keine erkennbaren Vorteile mehr gewonnen werden können. Man spricht dann von der Standardevolutionsstrategie.

Die Frage nach optimalen Werten für $\mu, \rho, \lambda$ bleibt offen. Diese Werte sind extrem problemabhängig, und können nicht pauschal angegeben werden. In [35] gibt Rechenberg das Verhältnis von $\mu / \lambda$ zwischen $1 / 5$ und $1 / 3$ an. Diese Werte sind für einige ,gutartige“ Qualitäts- und Testfunktionen sinnvolle Richtwerte. Allerdings versagen diese Werte bei realen Problemstellungen teilweise völlig.

Ein wesentliches Merkmal aller ES ist die adaptive Schrittweitenregelung der Mutation. Die Mutation kann man natürlich wie im GA durchführen. Das Problem dabei wäre, daß die Elemente des Vektors stets in einem eingeschränkten Rahmen verharren würden, d.h. es würde stets derselbe Quadrant des n-dimensionalen Parameterraums nach einer optimalen Lösung abgesucht werden. So ein Vorgehen würde das Auffinden eines globalen Optimums jedoch erheblich erschweren, wenn nicht gar unmöglich machen. Aus diesem Grunde muß ein wichtiger Mechanismus zur Durchführung der Mutation hinzugezogen werden, den man adaptive Schrittweitenregelung nennt.

Die wesentlichen Mechanismen der ES werden in [35], [37] und [38] ausführlich behandelt.Für 
die „Nachbehandlung“ der Paretofront nutze ich die $(\mu+\lambda)$-ES. In Worten bedeutet das, daß die Eltern (Ausgangsprofile) und die Nachkommen (die mutierten Profile) gemeinsam bewertet werden und die jeweils $\mu$-Besten als neue Eltern (Ausgangsprofile) überleben und aus diesen dann wieder die neue Generation hervorgeht.

In der ES werden ebenfalls Vektoren reeller Zahlen definiert. Sei $p^{i}$ das i-te Elter (diese Ausdrucksweise ist in der Literatur evolutionärer Algorithmen durchaus gebräuchlich)

$$
p^{i}=\left(x_{1}^{i}, \ldots, x_{n}^{i}\right) \quad x \in \mathfrak{R} ; \quad i, n \in N
$$

Die Anzahl der Ausgangsprofile (Eltern) ist $\mu$. Die $\lambda$ Nachkommen werden durch Mutation aus der Elterngeneration berechnet. Über die Elternvektoren wird die Streuung berechnet:

$$
\sigma_{j}=\sqrt{\frac{1}{\mu} \cdot \sum_{i=1}^{\mu}\left(x_{j}^{i}-m_{j}\right)} \quad 1 \leq j \leq \mu
$$

mit $m$ als Mittelwert der $j$-ten Streuungskomponente des Vektors $p^{i}$. Auf diese Weise entsteht ein Streuungsvektor. Die Streuung kann für jede Komponente unterschiedlich sein. Für die Änderungen der Profilparameter verwende ich die um $m=0$ liegende Gaußkurve,

$$
f(\sigma)=\frac{1}{\sqrt{2 \cdot \pi}} \cdot e^{-\frac{(x-m)^{2}}{2 \cdot \sigma^{2}}}
$$

mit der Streuung $\sigma$. Mit der angegebenen Gaußkurve ist es möglich, die Änderung jedes Profilparameters zwischen $0 \%$ und $4 \%$ zu begrenzen. In Abhängigkeit der erzielten Erfolge (Erfolg = Überschreiten der Paretofront) kann der Streuungsparameter angepaßt werden. Der Effekt ist, daß bei vielen Verbesserungserfolgen der Streuungsparameter künstlich vergrößert wird, d.h, daß ein größeres Gebiet abgesucht werden wird. Entsprechend wird der Streuungsparameter künstlich verkleinert, wenn man sich schon nahe dem Optimum befindet. Da in der Umgebung der Paretofront Profile ähnlichen Leistungsbereichs auch ähnliche Profilparameter haben, ist die Streuung in dem Fall ohnehin schon klein. Das führt zur bekannten 1/5 Erfolgsregel. „Der Quotient aus den erfolgreichen Mutationen zu allen Mutationen sollte mindestens 1/5 betragen. Ist der Quotient größer als 1/5 sollte die Streuung vergrößert werden; ist der Quotient geringer, sollte die Streuung verringert werden“.

$$
\begin{array}{ll}
\sigma(t+n)=c \cdot \sigma(t) & \text { wenn } \mathrm{p}>\frac{1}{5} \\
\sigma(t+n)=d \cdot \sigma(t) & \text { wenn } \mathrm{p}<\frac{1}{5} \\
\sigma(t+n)=\sigma(t) & \text { wenn } \mathrm{p}=\frac{1}{5}
\end{array}
$$

mit $c=1.22$ und $d=0.82$ und $p$ Erfolgsparameter. 
Die Streuung soll in der Generation $t+n$ um den Faktor 1.22 vergrößert werden, wenn die Erfolgsquote in $n$-Generationen im Schnitt größer als $1 / 5$ ist. Liegt die Erfolgsquote unter 1/5, dann wird die Streuung um den Faktor $d$ verkleinert. Bei einer Erfolgsquote von 1/5 bleibt die Streuung unverändert.

\subsection{Einfachoptimierung mit Evolutionären Methoden}

Die oben beschriebenen evolutionären Methoden sind Optimierungsmethoden. Diese haben grundsätzlich andere Eigenschaften und Fähigkeiten als Methoden, die nach dem Gradientenverfahren arbeiten. Der wichtigste Unterschied liegt in der prinzipiellen Gefahr, nach Auffinden eines lokalen Optimums einer Funktion dort zu verharren und die Suche abzubrechen, denn das eigentliche Ziel ist das globale Optimum zu finden. Genau dieses Verhalten aber ist ein fundamentales Manko der auf Gradienten basierenden Methoden. Für die evolutionären Methoden ist diese Gefahr deutlich reduziert. Durch verteiltes paralleles Suchen ist das Auffinden eines globalen Optimums sehr viel wahrscheinlicher.

Dieses Verhalten gilt sowohl für Optimierungen nach einem Parameter als auch für Optimierungen nach mehreren Parametern. Man kann sich leicht vorstellen, daß eine Gradientenmethode um so schlechter abschneidet, je mehr Parameter zu optimieren sind.

Wird nach mehreren Parametern gleichzeitig optimiert, wird dieser Algorithmus in der Literatur als Multiobjective GA (MOGA) oder als multimodaler GA bezeichnet. Entsprechend sind die Bezeichnungen für Einparameteroptimierungen gewählt - Singleobjective GA (kurz SOGA). Ich nenne es hier Einfach- und Mehrfachoptimierung und wähle aufgrund der Kürze die Bezeichnungen SOGA / MOGA.

Ein Beispiel für eine Einfachoptimierung ist das Auffinden einer Profilkontur, die eine vorgeschriebene $\mathrm{C}_{\mathrm{p}}$-Verteilung reproduzieren soll. Also ein inverser Profilentwurf durch Vorgabe einer $\mathrm{C}_{\mathrm{p}}$-Verteilung. In diesem Fall wäre die $\mathrm{C}_{\mathrm{p}}$-Verteilung das Kriterium, nach der eine Geometrie zu optimieren wäre. Die Geometrie der Profilkontur selbst wäre zwar durch viele Parameter definiert, aber für die Bestimmung, ob es sich um eine Einfach- oder Mehrfachoptimierung handelt, ist die Anzahl der Kriterien, nach denen optimiert werden soll, entscheidend.

So ist also das Ergebnis einer Einfachoptimierung ein Punkt (ein einzelner Wert). Das Ergebnis einer Mehrfachoptimierung nach zwei Kriterien gleichzeitig wäre eine Linie, und eine Mehrfachoptimierung nach drei Kriterien wäre eine Fläche. Da innerhalb dieser Arbeit, wie vorne beschrieben, im wesentlichen die Gleitzahl (also Auftrieb geteilt durch Widerstand) optimiert werden soll, entspricht das einer Mehrfachoptimierung nach zwei Kriterien. In der Literatur wird das als Pareto-Optimierung bezeichnet. Aufgrund der Wichtigkeit der Pareto-Optimierung wird diese im nächsten Abschnitt ausführlich besprochen. Die Einfachoptimierung ist praktisch ein Teilgebiet der Mehrfachoptimierung und bedarf deshalb hier keiner ausführlichen Beschreibung. 


\subsection{Mehrfachoptimierung mit Evolutionären Methoden}

Mit einer Mehrfachoptimierung wird hier die gleichzeitige Optimierung nach mehreren Kriterien bezeichnet. Wird speziell nach mindestens zwei Kriterien optimiert, so spricht man von Pareto-Optimierung. Einen gutes Beispiel für die Pareto-Optimierung gibt Shigeru Obayashi [24]. Das Ziel der Pareto-Optimierung nach zwei Kriterien ist es, das Optimum aus beiden Kriterien gleichzeitig zu erhalten. Das bedeutet, daß keine Größe im Ergebnis weiter verbessert werden kann, ohne eine andere Größe zu verschlechtern. Dies ist die Grundaussage der ParetoOptimierung.

Mathematisch läßt sich die Pareto-Optimierung wie folgt beschreiben.

Sei $p_{i}=\left(x_{i}, y_{i}\right)$ ein Satz Parameter, bestehend aus $x_{i}$ und $y_{i}$. und sei $f_{l}=\left(p_{i}\right)$ eine zu optimierende Funktion von $p_{i}$, und $f_{2}$ eine andere zu optimierende Funktion von $p_{i}$ und $F=\left(f_{1}, f_{2}, \ldots, f_{n}\right)$ sei der komplette Satz der Optimierungsfunktionen.

Man sagt, $p_{i}$ wird durch $p_{j}$ beherrscht ( $p_{j}$ ist im Range höher als $p_{i}$ ), wenn gilt:

$$
f_{1}\left(p_{i}\right) \leq f_{1}\left(p_{j}\right) \wedge f_{2}\left(p_{i}\right) \leq f_{2}\left(p_{j}\right), \text { mit } f\left(p_{i}\right) \neq f\left(p_{j}\right)
$$

$p_{j}$ hat den Rang 1, wenn alle $p_{i}$ die Ungleichung (8) erfüllen. Dann bildet der Parametersatz $p_{j}$ eine pareto-optimale Lösung, d.h. eine Lösung, die nicht in einer Funktion $f_{1}$ verbessert werden kann, ohne eine andere Funktion $f_{2}$ zu verschlechtern. Die Funktion $F$ bildet den pareto-optimalen Satz in dem gegebenen Funktionenraum. Die Bedeutung dieser Aussage sei anhand der folgenden Skizze grafisch verdeutlicht.

\section{Pareto-Optimierung \\ Ranking}

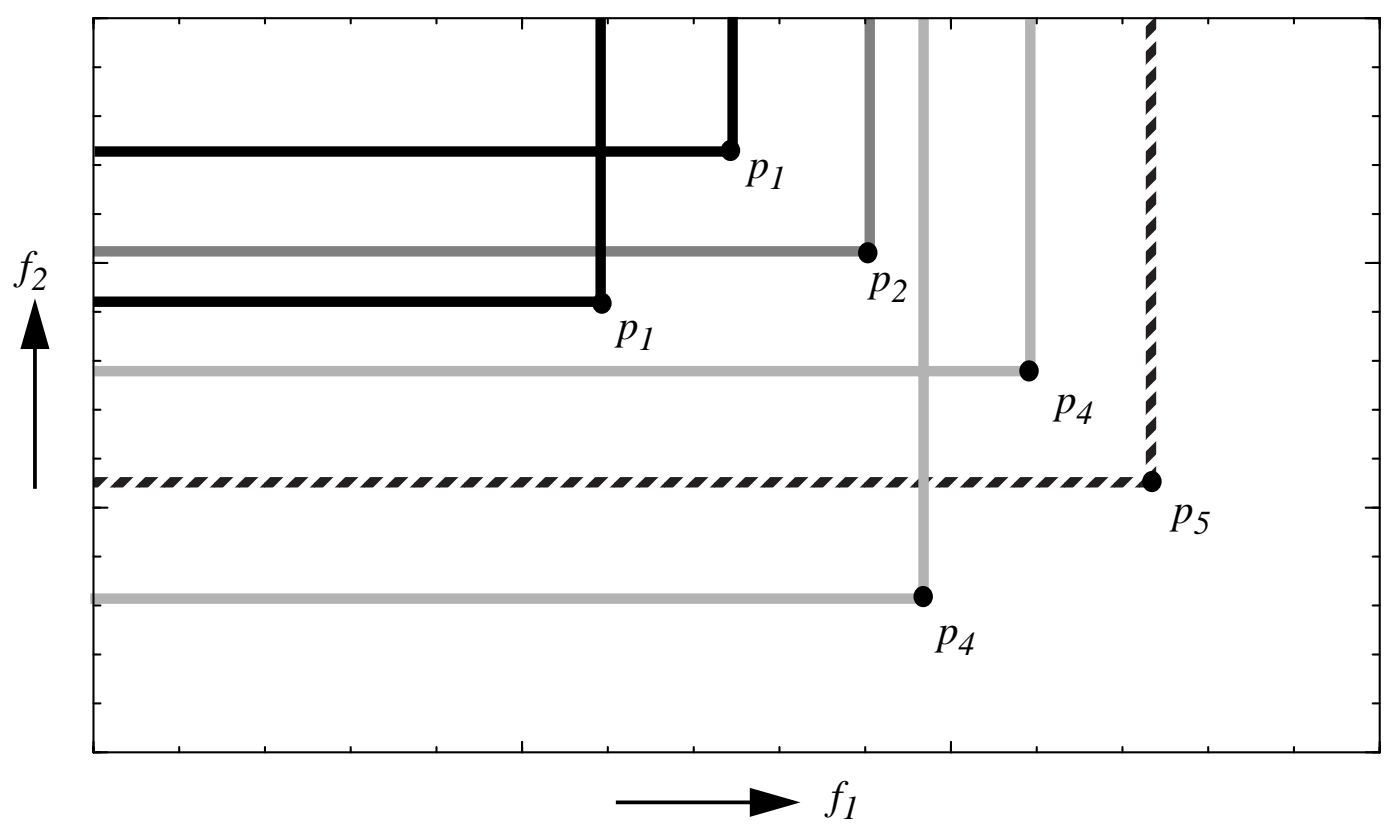

Abb. 4 Skizze der Pareto-Optimierung. 
In diesem Beispiel ist das Bestreben der Optimierung einen möglichst kleinen Wert für $f_{l}$ bei einem maximalen Wert für $f_{2}$ zu erhalten. Da beide Funktionen von demselben Parametersatz $p$ abhängen, kann eine Verbesserung der einen Funktion nicht ohne Verschlechterung (oder zumindest eine Veränderung) der anderen Funktion durchgeführt werden. Das Ziel ist es, die besten Werte beider Funktionen gleichzeitig zu erreichen (hier möglichst kleines $f_{l}$ bei möglichst großem $\left.f_{2}\right)$. Bei einem Vergleich zweier Wertepaare $\left(f_{1}, f_{2}\right)$ liegt also stets jenes Wertepaar besser, welches relativ zum anderen Wertepaar im 2. Quadranten liegt.

Für das Ranking werden nun für alle Parametersätze $p$ die Wertepaare $\left(f_{1}, f_{2}\right)$ bestimmt. Alle Parametersätze werden mit dem Rang 1 initialisiert ( $p_{i}$, der Index bezieht sich hier auf den Rang). Für alle Parametersätze $p$ wird die Anzahl der Wertepaare $\left(f_{l}, f_{2}\right)$ bestimmt, die sich relativ zu diesem im 2. Quadranten befinden. Für jedes Wertepaar wird dann der Index $i$ (der Rang) des Parametersatzes $p_{i}$ um eins erhöht. So werden die Indizes der $p_{i}$ in der Skizze oben bestimmt. Hat jeder Parametersatz $p_{i}$ den Index 1, dann ist eine pareto-optimale Lösung für alle Parametersätze erreicht. Die Einhüllende aller Wertepaare $\left(f_{1}, f_{2}\right)$ bildet dann die sogenannte Paretofront.

Nehmen wir an, die Lösungspunkte in Abb. 4 repräsentieren den Auftrieb und Widerstand einer aerodynamischen Konfiguration (Profil oder Tragflügel). Das Erreichen der Paretofront sagt aus, daß es dann (bei gleicher Geometriedefinition) keine Konfiguration mehr geben kann, die in Auftrieb und Widerstand den Konfigurationen auf der Paretofront überlegen wäre. Mit Erreichen der Paretofront ist eine Grenze erreicht, die, bei gleicher geometrischer Definition der Konfigurationen, nicht mehr überschritten werden kann. Die physikalische Bedeutung der Pareto-Optimierung in Bezug auf aerodynamischen Auftrieb und Widerstand wird im folgenden Kapitel 5.4 behandelt.

Man mache sich an dieser Stelle klar, daß mit dem beschriebenen Vorgehen die in der Einleitung angestrebte Grenze zu erreichen wäre. Weiter wird hier schon offenbar, daß diese Grenze von der Flexibilität der Geometriedefinition der Konfigurationen wesentlich abhängt. So wird man jetzt schon vermuten, daß eine sehr flexible Geometriedefinition eine ,überlegene“ Paretofront reproduzieren wird gegenüber einer Geometriedefinition, die gewisse Einschränkungen hinsichtlich der geometrischen Möglichkeiten aufweist. Dieses wird im folgenden noch Gegenstand der Untersuchung sein.

\section{Fitneßwertberechnung}

Zusätzlich zur Bewertung eines Objekts durch das Ranking-Verfahren wird den Objekten ein Fitneßwert zugewiesen. Dieser Fitneßwert repräsentiert quasi die Überlebensfähigkeit eine jeden Objekts. Die Wirkung der Fitneßwerte ist durch das vorgelagerte Ranking-Verfahren in seiner Wirkung zwar beschränkt, aber nicht unerheblich. Denn viele Objekte gleichen Ranges sind nicht gleichwertig. So wird in zweiter Instanz eine Bewertung durch den Fitneßwert realisiert und sorgt für eine Ordnung von Objekten gleichen Ranges.

Die Abb. 4 erweckt den Eindruck, als würde die errechnete Paretofront letztendlich eine Linie sehr gleichmäßig verteilter Punkte sein. Dies ist jedoch nur in Ausnahmefällen gegeben. Wenn man keine besonderen Vorkehrungen trifft, dann sind die Punkte auf der Paretofront sehr unregelmäßig verteilt. Das hängt damit zusammen, daß in einer sehr frühen Generation Objekte mit 
hohem Fitneßwert das Bestreben haben, andere Objekte in ihrem Umkreis zu versammeln. Das ist grundsätzlich gewollt, denn Bereiche mit hohem Fitneßwert sollen genauer untersucht werden. Das geschieht durch eine Verdichtung der Objekte in diesem Bereich.

In Falle einer Pareto-Optimierung möchte man aber die Punkte möglichst gleichmäßig auf der Paretofront verteilt haben, d.h. man möchte den gesamten Bereich von Objekten bestimmt haben, der prinzipiell möglich ist. Dazu muß man eine besondere Technik einführen, die für diese Gleichverteilung von Punkten auf der Paretofront sorgt.

Die Qualitätsfunktion (Fitneßfunktion) muß nicht notwendig nur ein globales Maximum aufweisen. Es ist möglich, daß die Qualitätsfunktion eventuell mehrere nahezu gleichgroße Nebenmaxima aufweist. Der GA hat aber das Bestreben, nur ein Maximum aufzuspüren, und vernachlässigt möglicherweise die Nebenmaxima, wenn man keine besonderen Vorkehrungen trifft. Im Ergebnis der Qualitätsfunktion sind diese Nebenmaxima dann unbesetzt. Grundsätzlich möchte man aber alle Maxima der Qualitätsfunktion bestimmt haben.

Um dieses Verhalten eines GA zu umgehen, führt man das sogenannte Fitneß-Sharing ein. Das Fitneß-Sharing bewirkt, daß der Fitneßwert jedes Objektes nicht nur quasi seine Überlebensfähigkeit repräsentiert, sondern zusätzlich berücksichtigt, wieviel andere Objekte sich in unmittelbarer Umgebung befinden. Sind in der unmittelbaren Umgebung schon viele Objekte vorhanden, dann ist es nicht notwendig, dort weitere Objekte zu plazieren. Um eine weitere Anhäufung zu vermeiden, wird der ursprüngliche Fitneßwert künstlich weiter aufgeteilt, nämlich auf die Objekte, die sich in unmittelbarer Umgebung befinden. Das bewirkt eine flächenhafte regelmäßige Verteilung der Objekte im Lösungsraum. Auf die Paretofront bezogen heißt das, daß auf dieser Linie die Punkte regelmäßig verteilt sind und sich nicht alle Objekte auf einen kleinen Bereich drängen.

Die Fitneßwertberechnung wird nach dem Prinzip des Fitneß-Sharing realisiert. Sei: $d=d\left(p_{i}, p_{j}\right)$, der Abstand im Funktionenraum und $s(d)$ die typische Sharefunktion, mit:

$$
s(d)=\left\{\begin{array}{cl}
1-\left(\frac{d}{\sigma_{\text {share }}}\right)^{\alpha} & \mathrm{d}<\sigma_{\text {share }} \\
0 & \mathrm{~d} \geq \sigma_{\text {share }}
\end{array}\right.
$$

Dann ist der Fitneßwert durch:

$$
f_{s}\left(p_{i}\right)=\frac{f\left(p_{i}\right)}{\sum_{j} s\left(d\left(p_{i}, p_{j}\right)\right)}
$$

neu gegeben. In den Berechnungen wird $\alpha=0.1$ und $\sigma_{\text {share }}=0.02$ gesetzt. Diese Werte sind zunächst willkürlich gewählt, haben sich aber in den nachfolgenden Rechnungen gut bewährt. 


\section{Geometrische Grundlagen}

Die Geometriegenerierung im wissenschaftlichen Umfeld ist bis heute ein stark vernachlässigtes Thema. Das Schwergewicht der theoretischen Aerodynamik liegt auf der Berechnung von Strömungsfeldern. Hierzu gehören auch Berechnungen der Umströmung von aerodynamischen Profilen. Die Hauptaufgabe ist hier, das Strömungsfeld möglichst genau zu berechnen (simulieren) und besondere Effekte, wie Wirbel, Stöße und Strömungsablösung, genau vorherzusagen. Erste Voraussetzung für exakte Berechnungen sind exakte Randbedingungen. Diese können erfahrungsgemäß in großer Entfernung von der vorgegebenen Geometrie (dem umströmten Körper) gut vorgegeben werden. Die Gestaltung des Körpers selbst, in dessen unmittelbarer Nähe die Strömung von besonderem Interesse ist, und der selbst eine Randbedingung des Strömungsproblems darstellt, ist eine Herausforderung an die Möglichkeiten geometrischer Gestaltung. Dieses ist für Profile insbesondere dann der Fall, wenn es sich um lokale Optimierungen oder andere spezielle, lokal begrenzte Anpassungen an das Profil handelt.

Das berechnete Strömungsfeld ist abhängig von vorgegebenen Strömungsparametern und der vordefinierten Profilform. Das erklärte Ziel von Entwurfsrechnungen ist es, die auftretenden Effekte zu beschreiben und Rückschlüsse auf die Profilform zu gewinnen, um aerodynamisch ungünstige Effekte zu minimieren. Die auftretenden Effekte im Strömungsfeld sind aber ursächlich durch das Profil bedingt. Um leistungsmindernde Effekte zu vermeiden, sind also Korrekturen an dem Profil selbst erforderlich. Diese Korrekturen sind aber wiederum nur effektiv durchführbar, wenn die Geometrie des Profils in flexibler Weise handhabbar ist.

Bekannt sind Profile z.B. des NACA-Katalogs. Die dort wesentlichen Parameter lassen zwar eine Definition eines kompletten Profils zu, eine lokale, begrenzte Änderung des Profils ist mit dieser Methode jedoch nicht möglich.

Aerodynamische Profile beschreiben zunächst nur zweidimensionale oder im 3D-Raum zylindrische Formen. Da den heutigen Konfigurationen und Tragflügelauslegungen dreidimensionale Berechnungen vorausgehen, offenbart sich hier das Fehlen von Methoden zur Definitionen von vollständig analytisch definierten, dreidimensionalen Körpern. Eine dreidimensionale Konfiguration ist zur Zeit üblicherweise durch eine unterschiedliche Zahl von Raumpunkten und durch Splines definiert, die nur in wenigen Fällen im Hinblick auf eine aerodynamische Optimierung kontrolliert werden können. Ein feste Handhabe der Geometriedefinition und somit eine exakte Vorgabe der Geometrie ist in vielen Fällen notwendig, um überhaupt an Tragflügeln Optimierungen betreiben zu können.

\subsection{Analytisch definierte aerodynamische Profile}

Der Ausgangspunkt für die vollständige analytische Definition eines Tragflügels ist die analytische Definition des zweidimensionalen Profils. Der ursprüngliche Ansatz zur Profilgenerierung, auf der die im folgenden beschriebene Methode im wesentlichen aufsetzt, ist die bekannte Joukowsky-Transformation. Das theoretische Fundament der hier entwickelten Methode liefert die Theorie der konformen Abbildungen. In der Joukowsky-Transformation ist der Ausgangspunkt ein quer angeströmter Zylinder mit Kreisquerschnitt, für den die Abbildung gleich auch 
die reibungsfreie, inkompressible Umströmung modelliert. Um die an dem Zylinder gewonnenen Erkenntnisse auch auf anwendungsorientierte geometrische Körper, wie z.B ein Profil, übertragen zu können, begreift man die Profilebene als eine Ebene komplexer Zahlen ( $\mathrm{z}=\mathrm{kom}$ plex). Diese Ebene $\mathrm{Z}$ wird konform mit der Funktion:

$$
\zeta=z+\frac{1}{z}
$$

in die $\zeta$-Ebene abgebildet. Durch diesen Abbildungsmechanismus verformt sich der Kreisquerschnitt oder eine ähnliche Figur in ein aerodynamisches Profil. Wieder wird damit auch das reibungsfreie Strömungsmodell geliefert: Die Joukowsky Abbildung ist somit eine klassische, einfache Methode zum Studium idealisierter Profilströmungen; sie ist in der Folge auch für praxisorientierte Studien sinnvoll geworden. Die folgenden Betrachtungen klammern zunächst die Diskussion der Strömungsphänomene aus und beschränken sich auf die Definition der Geometrie des Profils. Die oben beschriebene Methode ist geeignet, eine einfache geometrische Figur in ein Profil zu überführen. Sie ist bekanntlich die Grundlage der klassischen Aerodynamik reibungsfreier (Potential-) Strömungen. Joukowsky erlaubt zunächst eine zweiparametrige Variation der Transformation des Kreises. Der Kreismittelpunkt ist aus dem Ursprung um dx, dy verschoben, siehe Abb. 5. Der Radius ist so gewählt, daß der Punkt (1.0, 0.0) von der kreisähnlichen Figur durchlaufen wird. Dieser Sachverhalt garantiert nach der konformen Abbildung eine scharfe Hinterkante. Die Abbildung des so gewählten Kreises ergibt in der abgebildeten $\zeta$ Ebene eine Profilform.

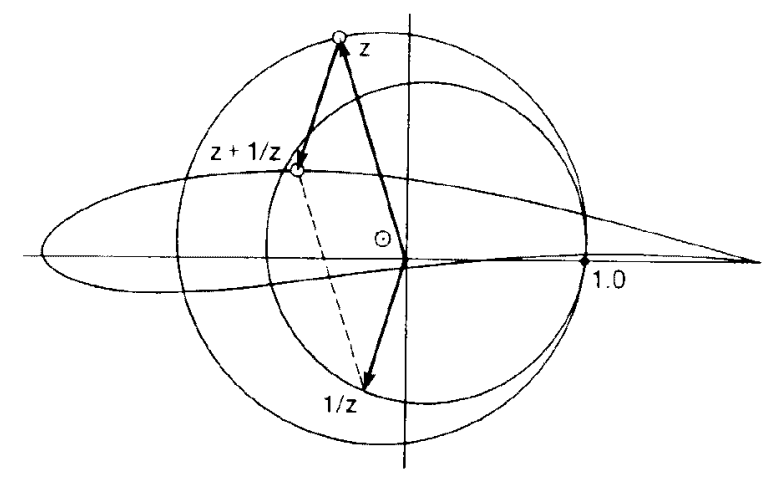

Abb. 5 Joukowsky-Transformation zur Definition aerodynamischer Profile.

Der Vorteil dieser Methode ist die Definition eines Profils mit einer minimalen Anzahl von Parametern. In diesem Fall ist das Profil durch zwei Parameter dx und dy definiert, wenn man die konforme Abbildung und die Bedingung, daß der Radius durch (1.0,0.0) verläuft, erfüllt. Der Nachteil besteht darin, daß mit nur zwei Parametern und der sonst festen Kreisform die mögliche Profilvariation bescheiden ist. Es handelt sich eben erst um den Beginn einer vollständig analytisch durchführbaren Methode. In der klassischen Aerodynamik werden durch Erweiterung der Abbildungsfunktion (11) beachtliche Erfolge in der Definition leistungsfähiger aerodynamischer Profile erreicht, siehe dazu die Darstellung aktueller Entwicklungen von R.T.Jones [17]. Neue Profile und ihre theoretische (reibungsfreie) Umströmung wurden mit diesen Methoden ermittelt. 
Hier soll nun, unter Verzicht auf das analytische Resultat für die Umströmung, die Festlegung einer kreisähnlichen Figur auf andere Weise erfolgen. Der Kreis soll durch eine allgemeine Funktion

$$
r=r(\varphi)
$$

definiert werden. Dieses Vorgehen trennt die Strömungsfeldberechnung von der eigentlichen Definition der Geometrie. Die Berechnung des Strömungsfeldes kann in einem nachfolgenden Verfahren geschehen, und dann auch auf kompressible und reibungsbehaftete Strömungsbedingungen erweitert werden.

Die Schwierigkeit für die Vorgabe einer Funktion wie in (12) gegeben, besteht dann darin, die Funktion auf einfache und elegante Weise zu beschreiben. Weiter muß die Anforderung gestellt werden, daß die Methode mit möglichst wenig Parametern auskommt. Zumindest sollen diese Parameter übersichtlich und in einer gut handhabbaren Form vorliegen. Eine weitere unverzichtbare Bedingung ist, daß das Profil in beliebiger Punktdichte vorliegen soll, um die Anforderung nach hoher Netzauflösung nachgeschalteter Strömungsrechenverfahren zu erfüllen. Zumindest letztere Bedingung ist praktisch nur durch analytische Funktionen zu erreichen, die jeden Punkt der Ebene definieren. Im folgenden soll die oben beschriebene Methode mit den Werkzeugen des Geometriegenerators nach Sobieczky [40] angereichert werden, um die dort erprobte Flexibilität auf die Profildefinition auszudehnen und in der Folge für dreidimensionale Anwendungen nutzbar zu machen.

Für eine allgemeine Definition eines deformierten Kreises $r(\varphi)$ bietet sich als Lösung an, diesen einfach in Segmente von $\varphi$ zu unterteilen. So gibt es dann eine Anzahl von Punkten $(\varphi, r)$, die von Segment zu Segment zu verbinden sind. Eine Möglichkeit ist, die Punkte stückweise durch Splines zu verbinden. Die so vorgegebene Kurve kann dann jedoch bei weit auseinanderliegenden Punkten schlecht kontrolliert werden und somit eine angestrebte Profilform nicht ausreichend genau vorgegeben werden. Aus diesem Grund wird hier auf analytische Funktionen in [40] zugegriffen, die eine bessere Kontrolle der Kurve ermöglichen. Die Wahl der Funktion aus diesem Katalog ist prinzipiell beliebig, diese sollte jedoch eine ausreichende Flexibilität beinhalten, verbunden mit einer starken Kontrolle des Kurvenverlaufs.

Die Abb. 6 zeigt die hier gewählte Vorgehensweise zur analytischen Profildefinition grafisch. Das Definitionsintervall der Kurven ist zunächst dimensionslos. Für eine stückweise Definition von $r(\varphi)$ muß auf die geforderten Größen skaliert werden. In diesem Fall werden die Werte der Abszisse auf Einheiten des Winkels in [rad] transformiert. Die Werte der Ordinate haben dann die Bedeutung des Radius.

Die horizontale Linie in Abb. 6 bei $r=1.0$ entspricht dem Einheitskreis. Nach der konformen Abbildung mit (11) wird der Einheitskreis auf eine Linie in dem Intervall $[-2,+2]$ abgebildet. Jede Abweichung von dieser konstanten Linie entspricht einem Abweichen der Konturlinie vom Einheitskreis. Genau dieser Sachverhalt wird hier für die Profildefinition genutzt. Es ist vorstellbar, die Profilkontur in der $\varphi$,r-Ebene nur durch einen einzigen Kurvenabschnitt (also nur durch eine Sektion, 'einsektional') vorzuschreiben. Günstiger ist es jedoch mindestens zwei Sektionen, jeweils für Profilober- und Unterseite zu verwenden, ('zweisektional', 'mehrsektio- 
nal'). Innerhalb dieser Arbeit werden maximal 4 Sektionen verwendet.

Es ist hier schon deutlich, daß die Geometriedefinition sehr flexibel angepaßt werden kann. Für besonders sensible Bereiche des Profils in einer Umströmung kann man in der $\varphi$,r-Ebene eine weitere Sektion einfügen, um diesen bestimmten Bereich gesondert zu behandeln. Die Stärke dieser Methode liegt in der Aneinanderkettung beliebig vieler Intervalle, die die Anforderung nach lokaler Anpassung des Profils erfüllen.

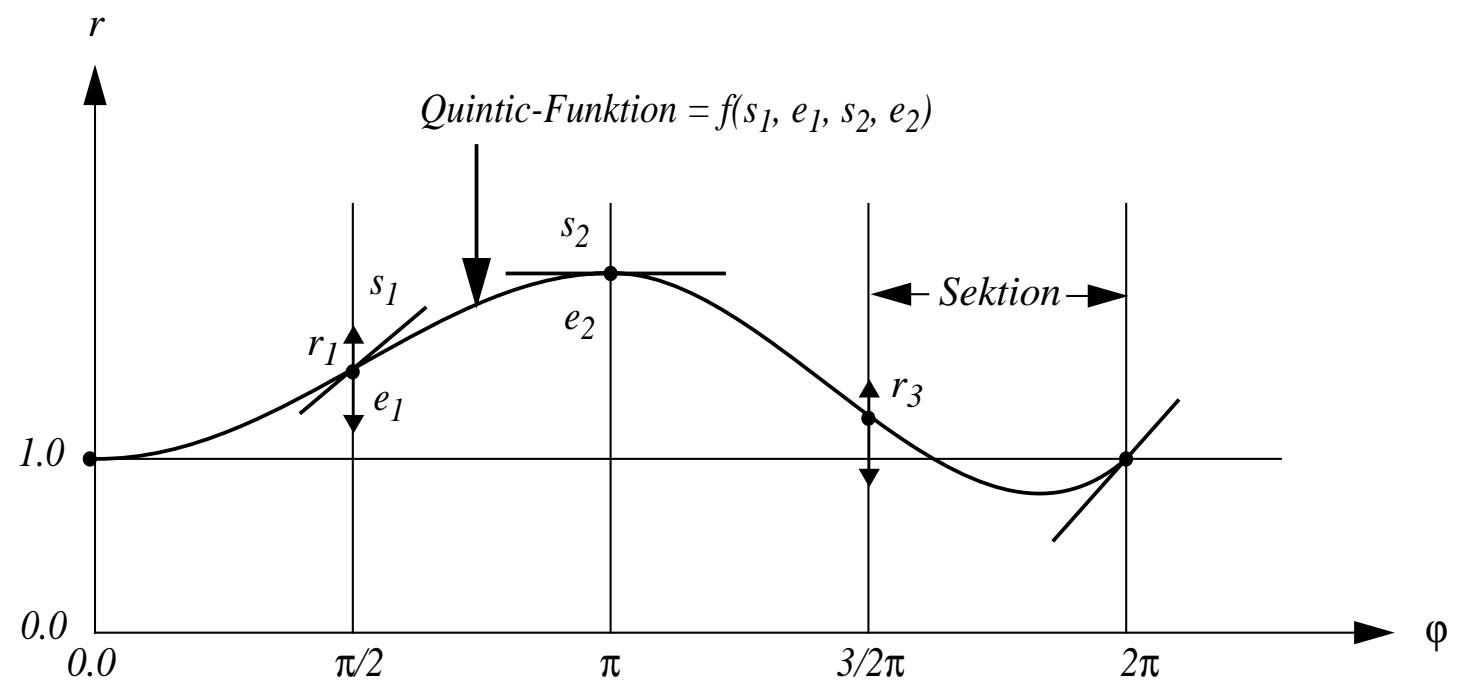

\section{Abb. 6 Verkettung von vier Quintic-Funktionen im $\varphi$-r-Diagramm.}

Der Kurvenverlauf innerhalb einer Sektion wird bei den Quintic-Funktionen (siehe Anhang) vollständig durch Vorgabe des Anfangs- und Endpunktes und deren erster und zweiter Ableitung in diesen Punkten beschrieben. Um Unstetigkeiten in Steigung und Krümmung zu vermeiden, ist es nötig, die Ableitungen an den Nahtstellen für aufeinanderfolgende Sektionen gleichzuhalten. So ist die Wahl der Parameter für jede Sektion eingeschränkt, weil die Parameter der benachbarten Sektion zu berücksichtigen sind. Aus dem gleichen Grunde ist die Vorgabe einer ausgewählten Funktion für alle Sektionen gleichzuhalten, da die Parameter $s_{1}, e_{1}$ und $s_{2}$, $e_{2}$ auch die gleiche Bedeutung für die Funktion zukommen muß. Weiter ist zu beachten, daß der Anfangspunkt einer Sektion dem Endpunkt der vorhergehenden Sektion entsprechen muß.

Hier wird sichtbar, wie sich die Geometriedefinition nahtlos in die genetische Codierung in (2) einfügt. Durch Vorgabe von mehreren Sektionen wird der genetischen Vektor einfach verlängert. An der Optimierungsmethode muß dabei nichts geändert werden. Die zu optimierenden Parameter beschränken sich auf die Parameter $s_{1}, e_{1}$ und $s_{2}, e_{2}$. Der Anfangs- und Endpunkt des gesamten Kurvenabschnitts $(\varphi=0.0,1.0)$ und $(\varphi=2 \pi, 1.0)$ ist konstant gehalten worden, damit das Profil eine geschlossene Kontur aufweist. Der Punkt $(\varphi=\pi, r)$ ist der Nasenpunkt des Profils. Aufgrund dieses charakteristischen Punktes ist auch dieser Punkt konstant gehalten worden.

Für die Vorgabe einer prozentualen Dicke eines Profils eignen sich die Punkte $\left(\varphi=\pi / 2, r_{l}\right)$ und $\left(\varphi=3 / 2 \pi, r_{3}\right)$. Die Lage dieser Punkte ist für die Definition einer relativen Dicke sinnvoll. Tat- 
sächlich basieren einige Fallstudien in dieser Arbeit auf einer relativen Profildicke von 17\%. Die Vorgabe der relativen Profildicke ist durch die oben genannten Punkte realisiert.

Innerhalb dieser Arbeit habe ich für ein erstes Studium der Geometrievariationen die Funktionen aus [40] entnommen. Diese können im Anhang eingesehen werden. Diese Funktionen unterscheiden sich im wesentlichen durch die Anzahl der Parameter, durch die sie bestimmt sind. Die Quintic-Funktion ist ein Polynom 5-ter Ordnung. Hier können erste und zweite Ableitungen an Anfangs- und Endpunkt vorgegeben werden. Als weitere Funktion nutze ich die CubicFunktion, ein Polynom 3-ter Ordnung. Die Vorgabe von zweiten Ableitungen fällt aufgrund der Definition der Cubic-Funktion weg . Für einen ersten Test nutze ich diese Funktionen mit einer, zwei und vier Sektionen.

\subsection{Analytische Tragflügelsektionen}

Die im vorigen Kapitel beschriebene analytische Profildefinition wird hier auf drei Dimensionen für die Tragflügelauslegung erweitert. Die Betrachtungen betreffen zunächst nur die Profilierung des Tragflügels. Im unteren Teil werden noch weitere geometrische Definitionen bezüglich Vorder- und Hinterkante und Verwindung besprochen.

Die analytische Profildefinition ist in Abb. 6 vorgestellt worden. Diese Geometriedefinition findet sich in Abb. 7 im Vordergrund wieder. Zusätzlich ist jetzt eine Methode zur Geometriedefinition in Spannweitenrichtung notwendig, an die dieselben Anforderungen gestellt werden muß wie an die bisherige Geometriedefinition. Denn im Zuge einer nachträglichen Tragflächenoptimierung müssen diese Parameter ebenfalls mit einem GA zu bearbeiten sein.

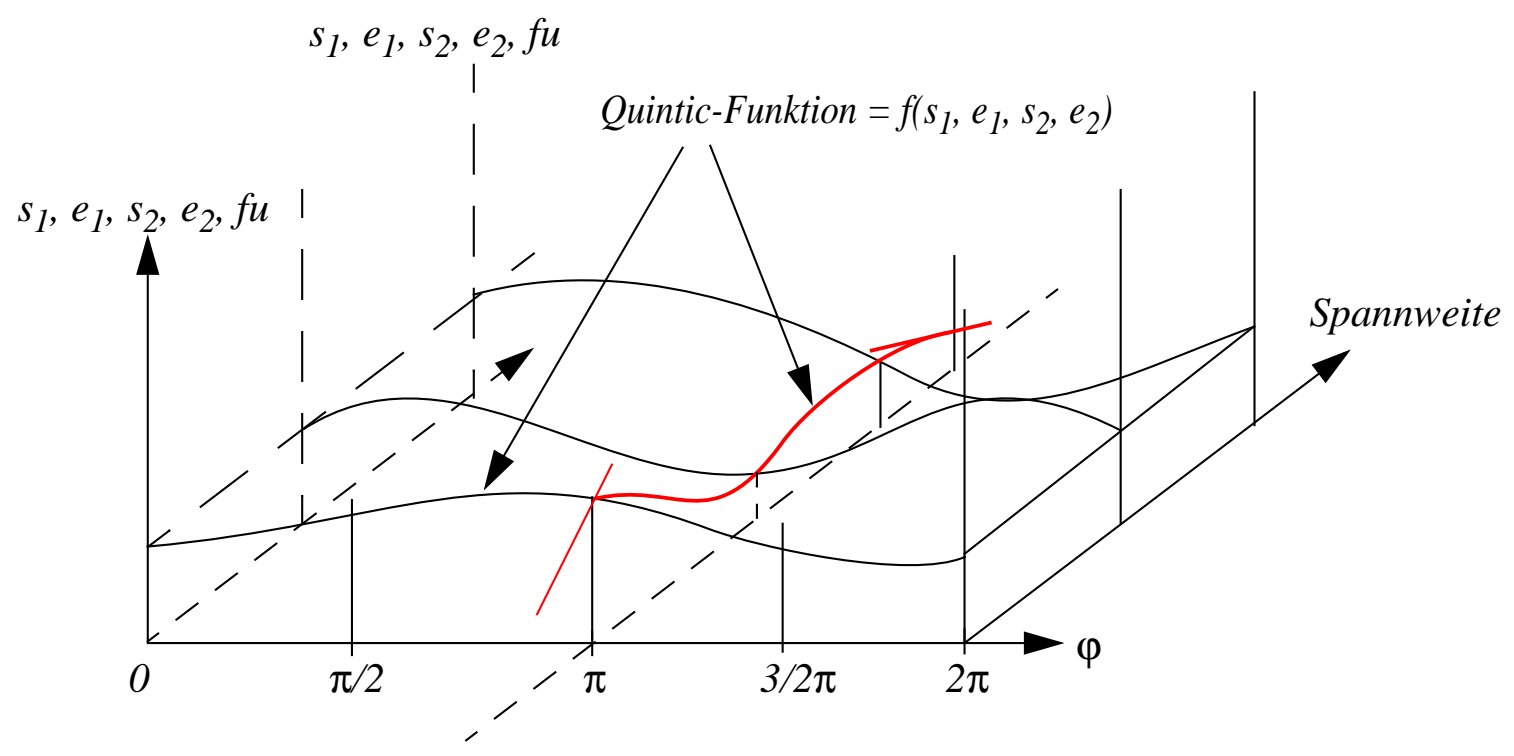

Abb. 7 Analytische Tragflügelprofilierung. 
Eine Definition, die direkt von den Querschnitten des Tragflügels abhängig ist, wäre für ein evolutionäres Optimierungsverfahren unbrauchbar. Denn die Geometrie ließe sich nur schwer oder gar nicht in eine genetische Codierung implementieren. Somit ist ein Beibehalten der Art und Weise der Geometriedefinition auch für die Spannweitenrichtung anzustreben. Die Konsequenz daraus ist, daß jetzt auch in Spannweitenrichtung mit denselben Funktionen Kurvenverläufe vorgeschrieben werden.

Die analytische Tragflügelprofilierung ist in Abb. 7 dargestellt. Zusätzlich zu der analytischen Definition der Profile in Strömungsrichtung wird die analytische Definition der Profile in der Spannweitenrichtung hinzugefügt. Für alle Parameter der Funktion (wie oben in Abb. 6 beschrieben) wird an allen Anfangs- und Endpunkten der Sektionen eine Parameterlinie in Spannweitenrichtung vorgegeben. Diese Kurve wird analytisch auf demselben Wege definiert (siehe Abb. 7 rote Linie) wie die Profilierung in Strömungsrichtung. Eine so definierte Kurve bestimmt nun exakt einen Parameter über die gesamte Spannweite. An die Stelle einzelner Parameter für die Definition zweidimensionaler Objekte treten in der dreidimensionalen Konfiguration Parameterlinien, die den Verlauf eines Parameters längs der Spannweite angeben. Für eine Optimierungsstrategie reicht es dann aus, den Verlauf der Parameterlinien zu variieren, um die Profilierung großflächig zu beeinflussen.

Diese Art der Geometriedefinition hat den Vorteil, daß die Optimierungsstrategie für Tragflügelkonfigurationen genauso durchgeführt werden kann, wie für den Fall der Profiloptimierung. Für eine EM sind nur Parameter einer Funktion zu optimieren. Die aus den Parametern resultierenden Kurven sind für die Optimierungsstrategie zunächst ohne Belang.

\subsection{Analytischer Flügelgrundriß}

Zusätzlich zu der Profilierung des Tragflügels sind weitere Kurven vorzugeben, um den Tragflügel vollständig zu definieren. Hierzu gehören der Flügelgrundriß (Planform), der Verlauf der Vorderkante und der Hinterkante, eine Kurve zur Vorgabe der Profilanstellung über die Spannweite (der Flügelverwindung) und eine Kurve zur Vorgabe der Dickenverteilung. Die parametrische Tragflügeldefinition über diese Linien ist von Sobieczky schon in [42] durchgeführt worden. So fügt sich hier die analytische Profildefinition in die analytische Tragflügeldefinition nahtlos ein. Darüber hinaus steht über die EM auch eine Methode zur Beurteilung dieser Art der Geometriedefinition zur Verfügung.

In Abb. 8 ist die geometrische Basis für eine elliptische Tragflügelgenerierung dargestellt. Über die Spannweite (hier die Abszisse) sind die Planform (Vorderkante, Hinterkante) und verschiedene weitere Parameterlinien schematisch aufgetragen. Dadurch wird der hohe Grad an Flexibilität der Geometriedefinition deutlich. Grundlage sind die oben ausführlich behandelte Profildefinition und die in Abb. 8 dargestellten Konturlinien für die Tragflügelkonfiguration. Alle diese Linien fasse ich unter dem Begriff Parameterlinien zusammen. Diese Parameterlinien berücksichtigen die wichtigsten geometrischen Größen der Körperkontur wie Profilierung, Anstellwinkel, Profildickenverteilung und Verlauf der Vorder- und Hinterkante. 


\section{Geometriedefinition durch Parameterlinien}

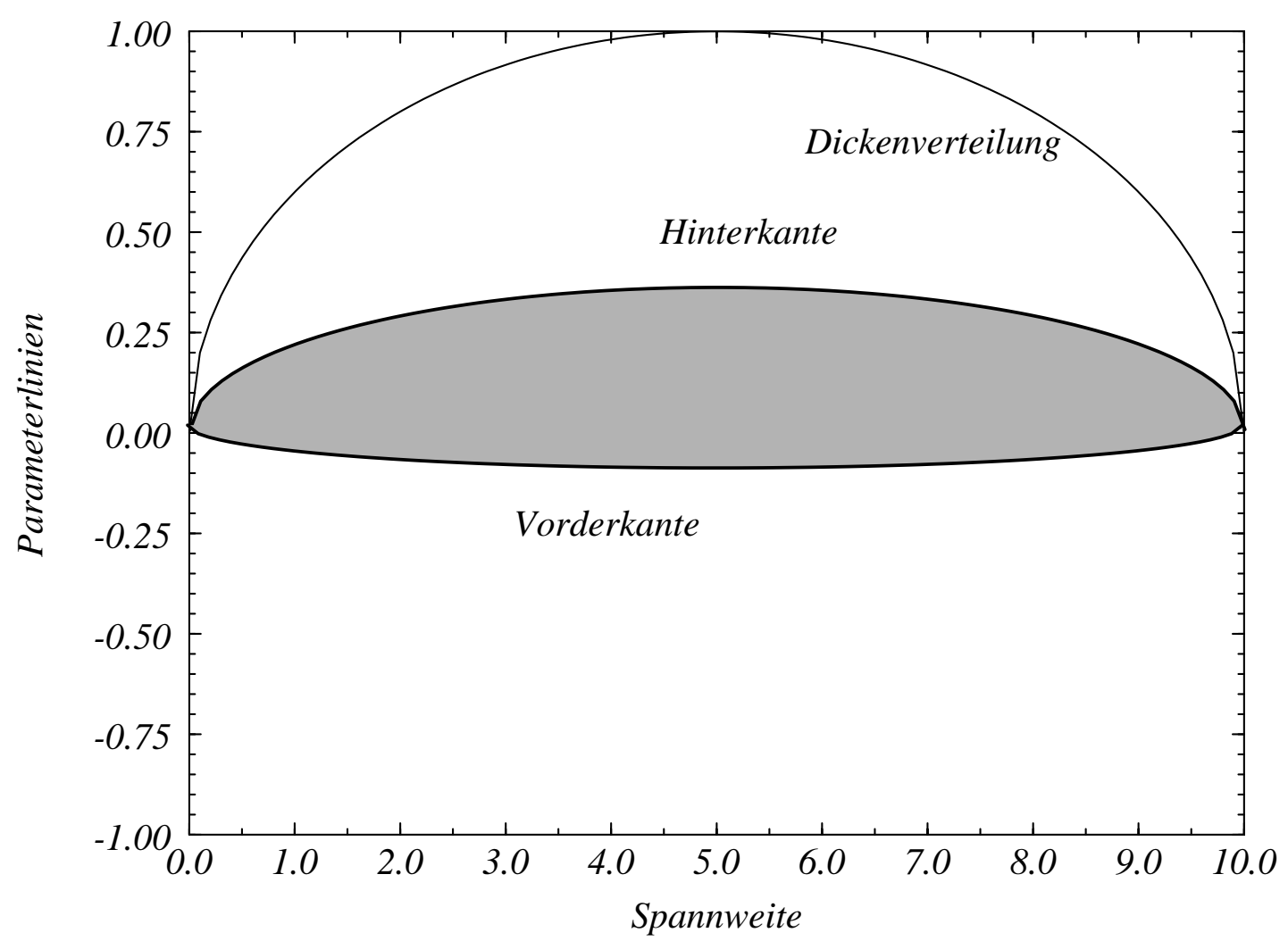

Abb. 8 Analytisch definierte Tragflügelkonturen: Flügelgrundriß, und Dicke nach einer manuellen Optimierung [42].

Hier wird offenbar, welche fundamentale Bedeutung dem abstrakten Abbildungsmechanismus, in der Genetik zwischen Genotypus und Phänotypus, zukommt. Darüberhinaus sehen wir hier den Vorteil, daß die Abbildungsvorschrift von Genotypus zu Phänotypus beliebig abstrakt sein darf und dennoch ein Optimierungsverfahren greifen kann. Übliche Optimierungsverfahren arbeiten, genetisch gesprochen, mit geringer Variation des Phänotypus und kommen möglicherweise zu keinen grundsätzlichen Aussagen bezüglich der Qualität eines Tragflügels. Es lassen sich dann nur Aussagen machen derart, daß der leicht variierte Tragflügel ein wenig besser ist als das ursprüngliche Ausgangsmodell. Es lassen sich so aber keine grundsätzlichen Grenzen ausloten, was hier ein Ziel der Optimierung ist.

Um die grundsätzlichen Grenzen ausloten, ist eine vollständige analytische Geometriedefinition Voraussetzung. Denn ohne den Parameterraum aller Möglichkeiten von Grund auf zu durchsuchen, lassen sich keine grundsätzlichen Rückschlüsse ziehen. Ein leichte nachträglich ausgeführte Verbesserung eines Tragflügelgrundmodells lotet die prinzipielle Grenze nicht aus, die aufgrund geometrischer Möglichkeiten besteht. Es ist notwendig, das Tragflügelgrundmodell in allen seinen geometrischen Möglichkeiten auszuleuchten, um dann in der Lage zu sein, grundsätzliche Gesetzmäßigkeiten festzustellen. 


\section{Physikalische Grundlagen}

In der vorliegenden Arbeit ist die Erhöhung der Wirtschaftlichkeit von Transport- und Verkehrsflugzeugen als vorrangiges Ziel genannt. Deswegen ist der physikalische Rahmen des Geschwindigkeitsbereichs interessant, in dem sich diese Flugzeuge bewegen: Der Bereich der hohen Unterschallgeschwindigkeit enthält komplexe transsonische Strömungsphänomene. Diese sind von dem Wechselspiel lokaler Unter- und Überschallgeschwindigkeiten an Profilen und Tragflügeln gekennzeichnet. Aufgrund der unterschiedlichen physikalischen Gesetzmäßigkeiten für Unter- und Überschall ist dieser mathematisch schwierig zu modellieren. Zur Berechnung einer Profil- oder Tragflügelumströmung existieren Verfahren, welche die physikalischen Gesetzmäßigkeiten unterschiedlich gut modellieren. Diese sind die Modelle der Potentialströmung, der Euler Gleichungen und der Navier-Stokes Gleichungen.

\subsection{Aerodynamisches Profil in transsonischer Anströmung}

In Abb. 9 und Abb. 10 ist ein schematischer Überblick gegeben. Dort sind die wesentlichen Bereiche, die das Strömungsverhalten charakterisieren, dargestellt. Ein geeignetes numerisches Verfahren zur Strömungsmodellierung sollte alle physikalischen Gesetzmäßigkeiten berücksichtigen. Diese Anforderung ist aus verschiedenen Gründen nicht immer zu erfüllen, denn der numerische Aufwand zur Lösung der entsprechenden Gleichungen wird sehr schnell groß. Insbesondere der Aufwand, der durch einen GA verursacht wird, gestattet zur Zeit nicht alle Strömungsphänomene in ihren Einzelheiten zu berücksichtigen, denn hier wird nicht ein einziges Profil nur einmal berechnet, sondern die Anzahl der zu berechnenden Profile bis zur Konvergenz in optimalen Formen erhöht sich auf einige tausend.

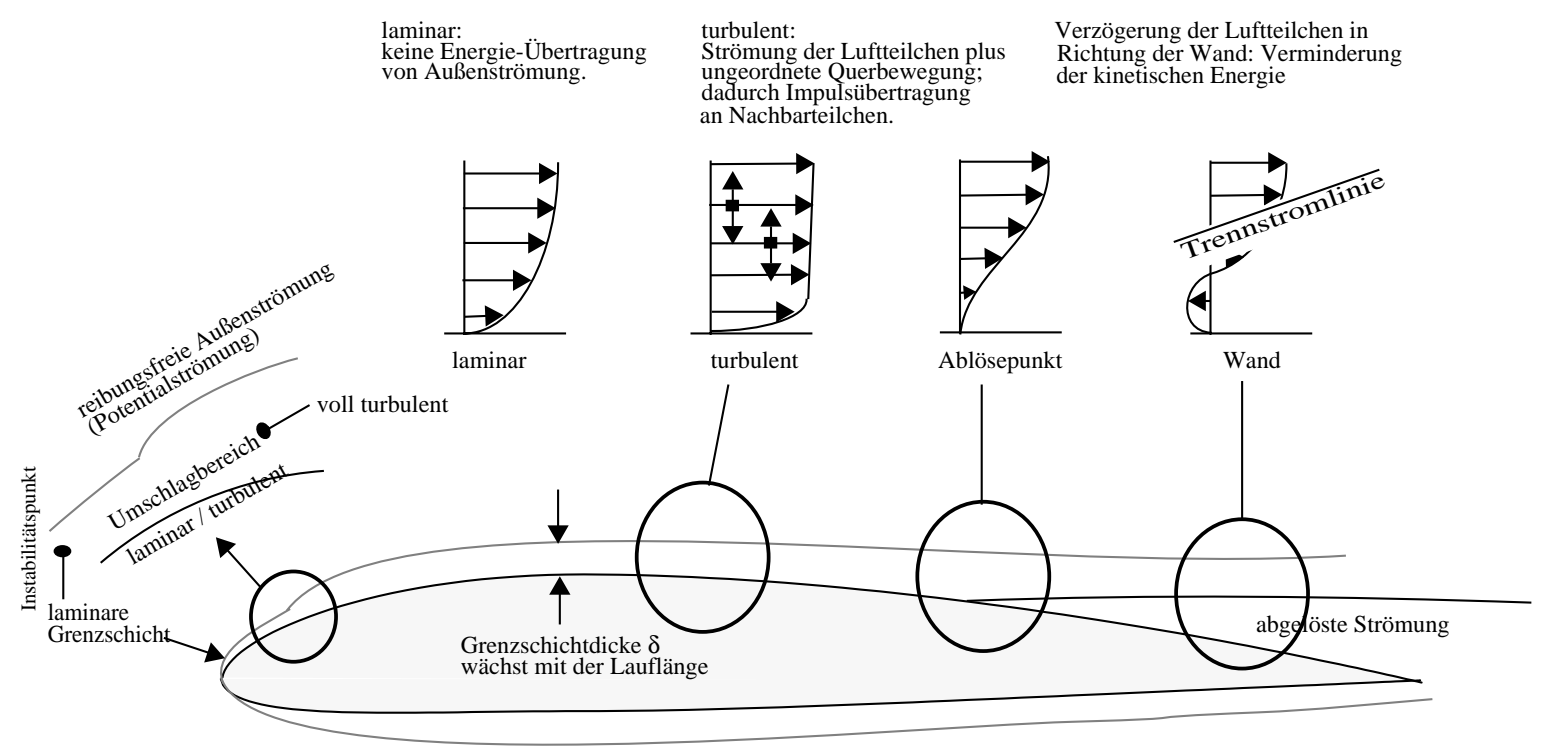

Abb. 9 Skizze: Profil in transsonischer Anströmung mit Reibungseffekten. 
Einen wesentlichen Einfluß auf die Umströmung des Profils übt die Form der Profilnase aus. Die Strömung wird hier am stärksten beschleunigt. Die Grenzschicht nahe der Profilnase ist zunächst laminar. Am Transitionspunkt (Instabilitätspunkt) werden Störungen in der Strömung angenommen, die weiter angefacht werden und schließlich zum turbulenten Umschlag der Grenzschicht führen. Die Grenzschicht (in Abb. 9 stark überhöht dargestellt) dickt sich zum Profilende hin stetig weiter auf. Sie liegt bis zum Ablösepunkt an dem Profil an. An diesem Punkt löst die Strömung ab, und bildet hinter dem Profil ein Totwassergebiet, innerhalb dessen die Profilform keinen Einfluß auf die Strömung mehr hat. Für alle Strömungsphasen ist in Abb. 9 der Geschwindigkeitsvektor skizziert. Hier ist das laminare Strömungsprofil an der Profilnase über die turbulente Phase an der Profiloberfläche bis hin zur Rückströmung nahe am Profil (hinter dem Ablösepunkt) zu erkennen.

Die Grenzschicht umfaßt den Bereich von einer Haftbedingung der Strömung an der Profilwand bis zu dem Punkt, an dem die Geschwindigkeit der Strömung den Wert der reibungsfreien Außenströmung erreicht. Außerhalb der Grenzschicht kann man die Strömung dann als reibungslos annehmen. Dies gilt allerdings nur für hohe Reynoldszahlen.

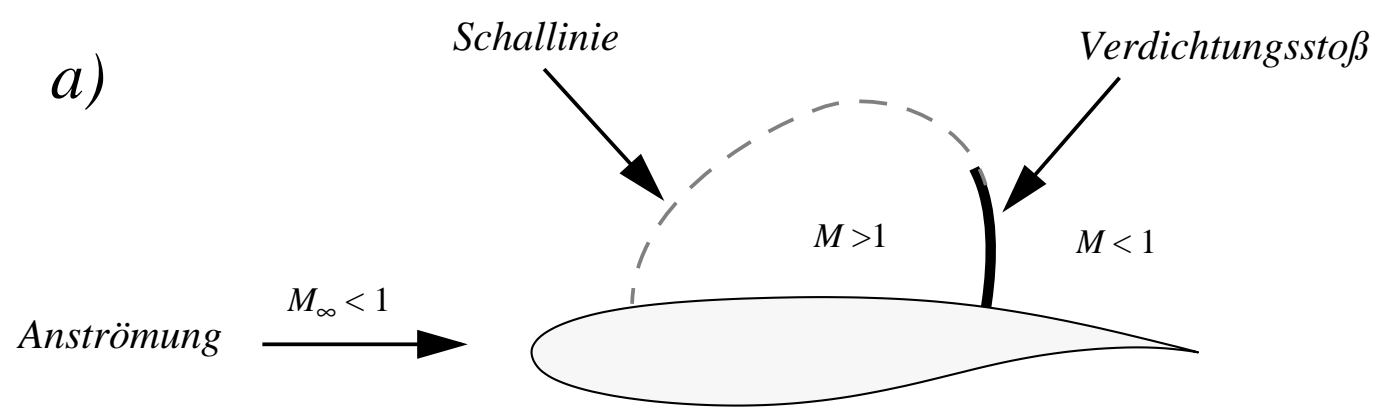

b)

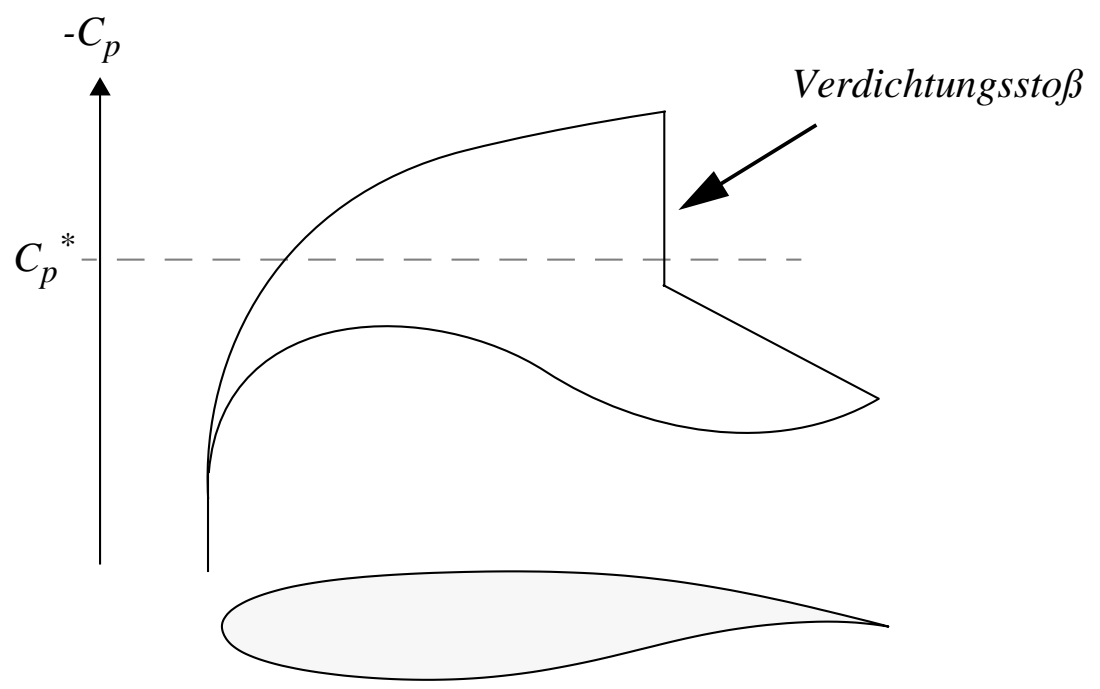
Abb. 10 a) Transsonische Anströmung eines Profils. Ausbildung eines Überschallge- bietes an der Profiloberfläche, mit Abschluß durch einen Verdichtungsstoß. b) Druckverteilung an der Profiloberfläche. 
Die Ablösung der Grenzschicht wird im transsonischen Bereich in der Regel durch einen Verdichtungsstoß verursacht. Wie in Abb. 10 zu erkennen bildet der Verdichtungsstoß den Abschluß des Überschallgebietes auf der Profiloberhälfte. Der Verdichtungsstoß bildet sich, wenn die Strömung nicht mehr stetig auf die veränderten Anströmbedingungen reagieren kann. Die Strömung verläuft unstetig mit einem Verdichtungsstoß. Der Verdichtungsstoß beeinflußt die Flugeigenschaften negativ: Einerseits bedingt er die Ablösung der Grenzschicht, andererseits führt er zur Entropiesteigerung. Damit wird der Widerstand erhöht und die Stabilität negativ beeinflußt. Diese Verluste könnten in gewissen Grenzen durch Änderungen an der Geometrie der Profils vermieden werden. Deswegen ist es vorrangiges Ziel bei dem Profil- und Tragflügelentwurf Verdichtungsstöße zu vermeiden oder deren Auftreten zu höheren Machzahlen zu verschieben. Zu diesem Zweck konstruiert man „superkritische Profile“. Hier tritt der Verdichtungsstoß dann erst bei höheren Machzahlen auf als sie dem Entwurfsbereich des normalen Reiseflugs entsprechen.

\subsection{Numerische Simulation der Strömung}

Für die Lösung der nichtlinearen Differentialgleichungen wie den Eulergleichungen und den Navier-Stokes-Gleichungen verwende ich den Standard-Code FLOWer [7]. Da die Einzelheiten der numerischen Verfahren innerhalb dieser Arbeit keine Rolle spielen, sondern nur als Werkzeug benutzt werden, gehe ich darauf nicht näher ein. Das in dieser Arbeit beschriebene Verfahren zur Optimierung der Geometrie ist nicht auf einen speziellen Strömungslöser festgelegt. Ein ebenfalls anerkannter Standard-Code ist der TAU-Code. Der wesentliche Unterschied zum FLOWer-Code ist, daß der TAU-Code mit unstrukturierten Netzen arbeitet. Dieser unstrukturierte Code hat Vorteile hinsichtlich der Flexibilität dreidimensionaler Netze. Der Nachteil ist, daß Lösungen auf unstrukturierten Netzen langsamer konvergieren als auf strukturierten Netzen. Da bei einem GA extrem viele Berechnungen anfallen, ist hier der schnellere Code attraktiver.

Die Grundlage aller Strömungslöser sind die Navier-Stokes-Gleichungen. Diese kann man für stationäre transsonische Strömungen ohne Volumenkräfte wie folgt beschreiben:

$$
v_{j} \frac{\partial v_{i}}{\partial x_{j}}=-\frac{1}{\rho} \frac{\partial p}{\partial x_{i}}+\frac{\partial}{\partial x_{j}}\left\{v\left(\frac{\partial v_{i}}{\partial x_{j}}+\frac{\partial v_{j}}{\partial x_{i}}-\frac{2}{3} \delta_{i j} \frac{\partial v_{k}}{\partial x_{k}}\right)\right\} \quad(i, j, k=1,2,3)
$$

Eine wesentliche Kennzahl ist die Reynoldszahl. Bei der hier gewählten transsonischen Flugmachzahl treten sehr hohe Strömungsgeschwindigkeiten auf. Die hohen Geschwindigkeiten sind gekennzeichnet durch große Werte der Reynolds'schen Zahl

$$
R e=\frac{q l}{v}
$$

Durch Einführen der Reynoldszahl ist eine Vereinfachung der Navier-Stokes-Gleichungen möglich. Für die Bereiche außerhalb der Grenzschicht kann man die Reibung vernachlässigen. Reibungseffekte treten vor allem in der Grenzschicht selbst auf. Die Grenzschicht beeinflußt al- 
lerdings die für die Aussenströmung effektive Konturberandung wesentlich.

Die Strömung außerhalb der Grenzschicht ist nicht wesentlich durch Reibungseffekte beeinflußt. Man kann hier die Reibung weitgehend vernachlässigen. Die Navier-Stokes-Gleichungen reduzieren sich dort auf die Euler-Gleichungen:

$$
-\frac{1}{\rho} \frac{\partial p}{\partial x_{i}}=v_{i} \frac{\partial v_{i}}{\partial x_{i}}+v_{j} \frac{\partial v_{i}}{\partial x_{j}}+v_{k} \frac{\partial v_{i}}{\partial x_{k}}
$$

Die Randbedingungen der Euler-Gleichungen unterscheiden sich von jenen der Navier-StokesGleichungen nur durch die fehlende Haftbedingung an der Profilwand. Durch die Eulergleichungen wird zwar die Reibung vernachlässigt, aber die Stöße im Strömungsfeld werden bei geeigneter Netzauflösung recht gut wiedergegeben. Da die Vermeidung der Stöße durch geometrische Manipulation das zentrale Thema dieser Arbeit ist, wurden in den hier beschriebenen Studien die Eulergleichungen zur Lösung der Strömungsprobleme herangezogen. Schon oben habe ich erwähnt, daß bei ,günstiger“ Druckverteilung nach Euler die Grenzschicht wenige negative Effekte aus dem Strömungsfeld aufnimmt. Daher ist zu erwarten, daß die Grenzschicht ein solides Verhalten zeigt und nicht vorzeitig am Profil ablöst.

Stoßfreie und reibungsfreie inkompressible Strömungen können durch ein Potentialverfahren gelöst werden. Die zugehörigen Potentialgleichungen sind hier angegeben:

$\frac{\partial}{\partial x_{1}}\left(\rho \cdot \frac{\partial \phi}{\partial x_{1}}\right)+\frac{\partial}{\partial x_{2}}\left(\rho \cdot \frac{\partial \phi}{\partial x_{2}}\right)+\frac{\partial}{\partial x_{3}}\left(\rho \cdot \frac{\partial \phi}{\partial x_{3}}\right)=0$, mit $q=\operatorname{grad}(\phi)$ und $\rho=\rho(q)$

In der Potentialtheorie werden die Profilumströmungen drehungsfrei behandelt, d.h. die mathematische Rotation des Vektorfeldes muß identisch Null sein. Das Verfahren versagt, wenn durch Stoßwellen Wirbelstärke induziert wird. Die damit verbundene Entropieerhöhung kann nicht mit den Potentialgleichungen erfaßt werden. Die Potentialgleichungen liefern für die Praxis brauchbare Ergebnisse, wenn keine schweren Stöße oder vollständige Stoßfreiheit vorliegen. Sind in dem Strömungsfeld Stöße zu erwarten und wird bei hohen Reynoldszahlen gerechnet, so sollten mindestens die Eulergleichungen verwendet werden.

\subsection{Druckverhältnisse am Profil}

Den wesentlichen Aufschluß über die Güte eines Profils gibt die Druckverteilung. Diese ist durch die Geometrie des Profils und die Anströmungsverhältnisse bedingt. In der dimensionslosen Darstellung Abb. 10 wird als Konvention der Druckbeiwert (Druckkoeffizient) mit negativem Vorzeichen über dem Profil aufgetragen. 


$$
c_{p}=\frac{p-p_{\infty}}{\frac{\rho}{2} \cdot q_{\infty}^{2}}
$$

In der freien Anströmung ist somit $c_{p}=0$. Für ein Profil ergeben sich für die Saug- und die Druckseite zwei Kurven, deren geschlossenes Integral dem Auftrieb entspricht, siehe Abb. 10b. In Abb. 21 und Abb. 33 sind gerechnete Druckverteilungen dargestellt. Zusätzlich wird das kritische $\mathrm{C}_{\mathrm{p}}{ }^{*}$ in diese Grafiken als Linie übernommen: Das Erreichen des kritischen Druckbeiwertes zeigt an, daß in diesem Punkt die lokale Machzahl $M=1$ erreicht wird. Für die Beurteilung der Strömungsqualität ist diese Darstellung sehr hilfreich.

\subsection{Optimierung zur Paretofront}

Der Mechanismus der Pareto-Optimierung ist in Kapitel 3.6 beschrieben. Welche Bedeutung kommt den Objekten (Profilen oder Tragflügeln) auf der Paretofront zu? Setzt man voraus, daß auch in der aerodynamischen Problemstellung, die in dieser Arbeit behandelt wird, die EM das geeignete Mittel zur Optimierung sind (und die folgenden Ergebnisse lassen kaum einen anderen Schluß zu), so entspricht jeder Punkt der Paretofront einem optimal gestalteten Objekt.

Abb. 11 illustriert dies: Die Rechnungen ergeben eine Anzahl von aerodynamischen Profilen (oder Tragflügeln), welche unter den gegebenen Variationsmöglichkeiten der geometrischen Parameter nicht mehr verbessert werden können hinsichtlich gesteigertem Auftrieb und vermindertem Widerstand.

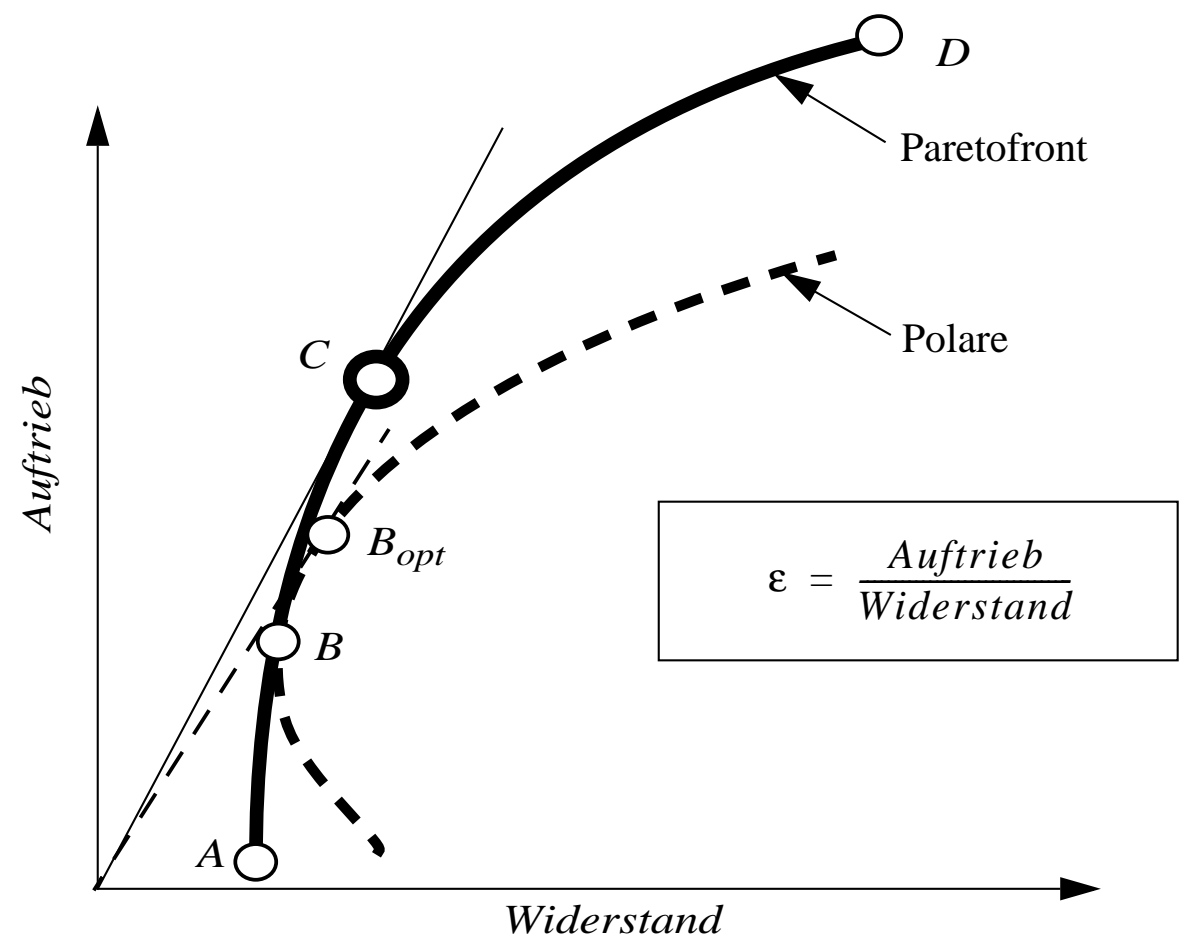

Abb. 11 Paretofront, Profilpolare und Gleitzahl $\varepsilon$. 
Zwischen einem Profil A mit kleinstem Widerstand, aber auch kleinstem Auftrieb, und einem Profil D mit größten Werten für beide Parameter, bringt als aerodynamisches Optimum ein Resultat C. Für dieses ist das Verhältnis Auftrieb/Widerstand, in der Aerodynamik als Gleitzahl $\varepsilon$ bezeichnet, ein Maximum.

Im Bilde ist weiterhin ein beliebiges Resultat B eingezeichnet, für welches auch die sog. Widerstandspolare als gestrichelte Linie skizziert ist: Auftrieb und Widerstand bei einer Nachrechnung dieses speziellen Profils (oder Flügels) bei unterschiedlichen Anstellwinkeln, abweichend vom Ausgangszustand „B“. Dabei wird grafisch die Tatsache deutlich, daß die optimale Gleitzahl $\left(\mathrm{B}_{\mathrm{opt}}\right)$ i.A. nicht auf der Paretofront liegt, dies ist nur für das optimale Resultat $\mathrm{C}$ der Fall.

Die Paretofront stellt somit die Einhüllende aller im Rahmen der Geometrievariation erreichbaren Konfigurationen dar und liefert als einzelnes Ergebnis auch die Konfiguration mit optimaler Gleitzahl. Einige Beispiele unterschiedlicher Paretofronten in Abhängigkeit der verwendeten geometrischen Funktionen werden in den folgenden Kapiteln dargestellt.

\subsection{Berechnung der Paretofronten auf einem Parallelcluster von Rechnern}

Zur Berechnung des Strömungsfeldes nutze ich den FLOWer-Code [7]. Dieser Strömungslöser ist ein Standard-Rechenprogramm des DLR (Deutsches Zentrum für Luft-und Raumfahrt e.V.) auf Basis moderner numerischer Methoden. Der Code selbst ist in der Lage, die stationären Euler- und Navier-Stokes-Gleichungen für zwei- und dreidimensionale Probleme zu lösen. Dieser Code verwendet strukturierte Mehrgitterverfahren und ist deswegen besonders effizient und schnell. Weiter verwendet dieser Code eine Blockstruktur. Damit können umfangreiche Konfigurationen in verschiedene Blöcke zerlegt werden und sind somit besser zu handhaben. Insgesamt erfüllt dieser Code die Anforderung an modernste numerische Verfahren und ist von hoher Flexibilität, deshalb wurde er hier ausgewählt.

In dem Code sind sämtliche Strömungsparameter wie Machzahl, Anstellwinkel, Schiebewinkel u.a. einzustellen. Da der Geschwindigkeitsbereich moderner Transportflugzeuge der hohe Unterschallbereich ist, wähle ich hier zunächst $M_{\infty}=0.707$ und einen Anstellwinkel für alle zweidimensionalen Rechnungen von $\alpha=1.46^{\circ}$. Die Machzahl und der Anstellwinkel sind so gewählt, daß ein Vergleich mit bereits existierenden Ergebnissen vollzogen werden kann. Insbesondere hinsichtlich der ebenfalls im folgenden behandelten dreidimensionalen Konfigurationsauslegung kommt der so gewählten Machzahl eine besondere Bedeutung zu. Neben der Nutzung der Eulergleichungen stellen diese beiden Parameter die Randbedingungen der folgenden Rechnungen dar.

Die Berechnung des Strömungsfeldes muß innerhalb des GA bei der Evaluation in jeder Generation für mehrere Profile erfolgen. Im folgenden Kapitel werden ich noch ausführen, daß zum Erreichen der voll auskonvergierten Paretofront mindestens 200 Profile eine Population bilden müssen. In dem ersten Schritt nach der Initialisierung des GA (siehe dazu Abb. 1) sind die Auftriebs- und Widerstandsbeiwerte von 200 Profilen zu bestimmen. In der darauffolgenden Generation sind jeweils nur die Profile neu zu berechnen, die von dem Algorithmus neu entworfen wurden und den Platz aussortierter schlechter Profile einnehmen. Wieviel das sind, hängt von dem Parameter der Überlebensrate (Generation Gap) des GA ab. Wenn man annimmt, daß je- 
weils die Hälfte der Population überlebt und ab der Konvergenz von 100 Profilen in den Rang 1 nur noch die Profile mit Rang > 1 neu berechnet werden, sind ca. 5000 mal die Strömung für verschiedene Profile durch die Eulergleichungen zu bestimmen. Diese Zahl ist nicht fest, sondern muß oft nach oben korrigiert werden.

In dem Umfeld der GA werden viele Techniken zur Parallelisierung bei den Berechnungen aufgezeigt. Der einzige zeitaufwendige Abschnitt hier ist die Berechnung der Strömungsfeldes. Aus den Werten für die Druckverteilung lassen sich dann die Auftriebs- und Widerstandsbeiwerte bestimmen. Das ist nach jedem neuen Profilentwurf durch den GA erforderlich.

Um diese hohe Zahl von Rechnungen ausführen zu können, bietet sich natürlich ein Parallelrechner mit einer hohen Anzahl von Prozessoren an. Da mir so ein Rechner leider nicht zur Verfügung steht, mußte hier eine andere Lösung gefunden werden. Die Lösung ist, den Workstation-Cluster des DLR in der Strömungsmechanik wie einen Parallelrechner zu nutzen. Der Workstation-Cluster besteht aus einer Zahl von ca. 70 voneinander unabhängigen Einzelrechnern, die über ein schnelles Netzwerk miteinander verbunden sind.

Die Verbindungen der Prozessoren über ein normales Netzwerk sind langsamer als die Verbindungen in einem echten Parallelrechner. Dennoch ist dies ein gangbarer Weg. Die gegenwärtige technische Ausstattung des Workstation-Clusters der DLR in allen Einzelheiten darzulegen, ist wenig sinnvoll, da die Entwicklung der Computer mit großen Schritten vorangeht. Was heute gültig ist, ist morgen vielleicht schon wieder überholt. Es sei aber erwähnt, daß beim heutigen technischen Stand ein Auskonvergieren der Paretofront für zweidimensionale Profile ca. 2 Tage dauert, bei dreidimensionalen Tragflügelkonfigurationen ca. 2 Wochen. 


\section{Ergebnisse aus der Anwendung der evolutionären Methoden}

Als Algorithmus zur Optimierung nichtlinearer Probleme haben die EM eine herausragende Bedeutung. Andere Optimierungsalgorithmen, die auf einer Gradientenmethode aufbauen, sind nur zweckmäßig, wenn man Verbesserungen eines Objekts durchführen will und die Richtung, in der die Parameter zu verändern sind, bekannt ist. Diese Richtung ist in der Regel a priori nicht bekannt. Bei der hier angestrebten Mehrfachoptimierung mit genetischen Algorithmen sind die EM klar favorisiert.

Eine Mehrfachoptimierung ist, wie oben schon dargelegt, eine Optimierung nach mindestens zwei verschiedenen Größen. Das besondere Interesse für die Optimierung gilt hier den Beiwerten für Auftrieb und Widerstand von aerodynamischen Konfigurationen. Der Zusammenhang der Geometriedefinition mit der aerodynamischen Güte einer Konfiguration soll hier bestimmt werden.

Prinzipiell ließen sich weitere Selektionsgrößen zusätzlich zu Auftrieb und Widerstand dem Optimierungsvorgang hinzufügen. Dieses ist aus der aerodynamischen Problemstellung auch geboten. Von Interesse wären auch Momentenbeiwerte, ein erweiterter Machzahlbereich und verschiedene Anstellwinkel. Jede weitere Optimierungsgröße erfordert eine Erhöhung der Anzahl zu berechnender Objekte (Populationsgröße) um eine Potenz. Wie im vorherigen Kapitel dargelegt, ist die zur Verfügung stehende Rechenkapazität nicht unerschöpflich. Und selbst die einfache Optimierung nach Auftrieb und Widerstand für reibungsfrei umströmte dreidimensionale Konfigurationen geht an die Grenze des rechentechnisch Machbaren. Aus diesen Grunde beschränke ich mich auf die oben genannten zwei Selektionsgrößen von Auftrieb und Widerstand.

In den vorherigen Kapiteln sind die dazu notwendigen Methoden vorgestellt worden. Ebenfalls ist eine parametrisierte Profil-und Tragflügeldarstellung entwickelt worden, die sich streng an den Optimierungsmethoden orientiert. Aufbauend auf ausgesuchten Funktionen und mit Hilfe weniger Parameter konnte eine effiziente Geometriedefinition für aerodynamische Profile und Tragflügel entwickelt werden. Damit ist ein wichtiger Schritt getan, denn diese Form der Geometriedefinition soll im folgenden als Grundlage einer speziellen Codierung für eine Optimierung durch einen GA dienen. Das Ziel dabei ist nicht eine lokale Verbesserung an einem Profil zu erreichen, sondern die geometrische Darstellung soll es erlauben eine grundsätzliche maximale Grenze aufzuspüren, und alle die Möglichkeiten, die durch die beschriebene Geometriedefinition gegeben sind, aerodynamisch auszuschöpfen.

Bevor ich mich den Ergebnissen zuwende, sollte die Wirkungsweise des GA nochmal verdeutlicht werden, denn aus den bisher beschriebenen Formeln, Verfahren und Definitionen ist möglicherweise noch kein klares Bild der Wirkungsweise des GA entstanden. Deswegen sei hier ein Einschub gestattet, der den Optimierungsmechanismus verdeutlicht.

\subsection{Die Wirkungsweise des Genetischen Algorithmus}

Geht man nach dem Prinzip der Mehrfachoptimierung zweier Parameter vor, so ist das Ergebnis der Anwendung des genetischen Algorithmus eine Kurve, die man als Paretofront be- 
zeichnet. Diese Front stellt die unüberschreitbare Grenze des Optimierungsvorganges dar. In Abb. 12 ist die Entwicklung zu dieser Front nach durchgeführten Rechnungen grafisch dargestellt.

Nach der Initialisierung der Population (hier der verschiedenen Profile) und der anschließenden Bewertung entstehen die $C_{l}, C_{d}$-Werte der Profile statistisch verteilt, in der Grafik repräsentiert durch die schwarzen Kreise. Zu diesem Zeitpunkt (in dieser Generation) ist noch kein Verbesserungsvorgang sichtbar. Dieser wird erst in der zweiten Generation deutlich. Aus der ersten Generation wird die Hälfte der Population beibehalten (rote Vierecke mit schwarzem Kreis), die schlechtere zweite Hälfte der ersten Generation wird aus der Population entfernt. An ihre Stelle treten die Nachkommen der zweiten Generation (rote Vierecke, ohne schwarze Kreise)

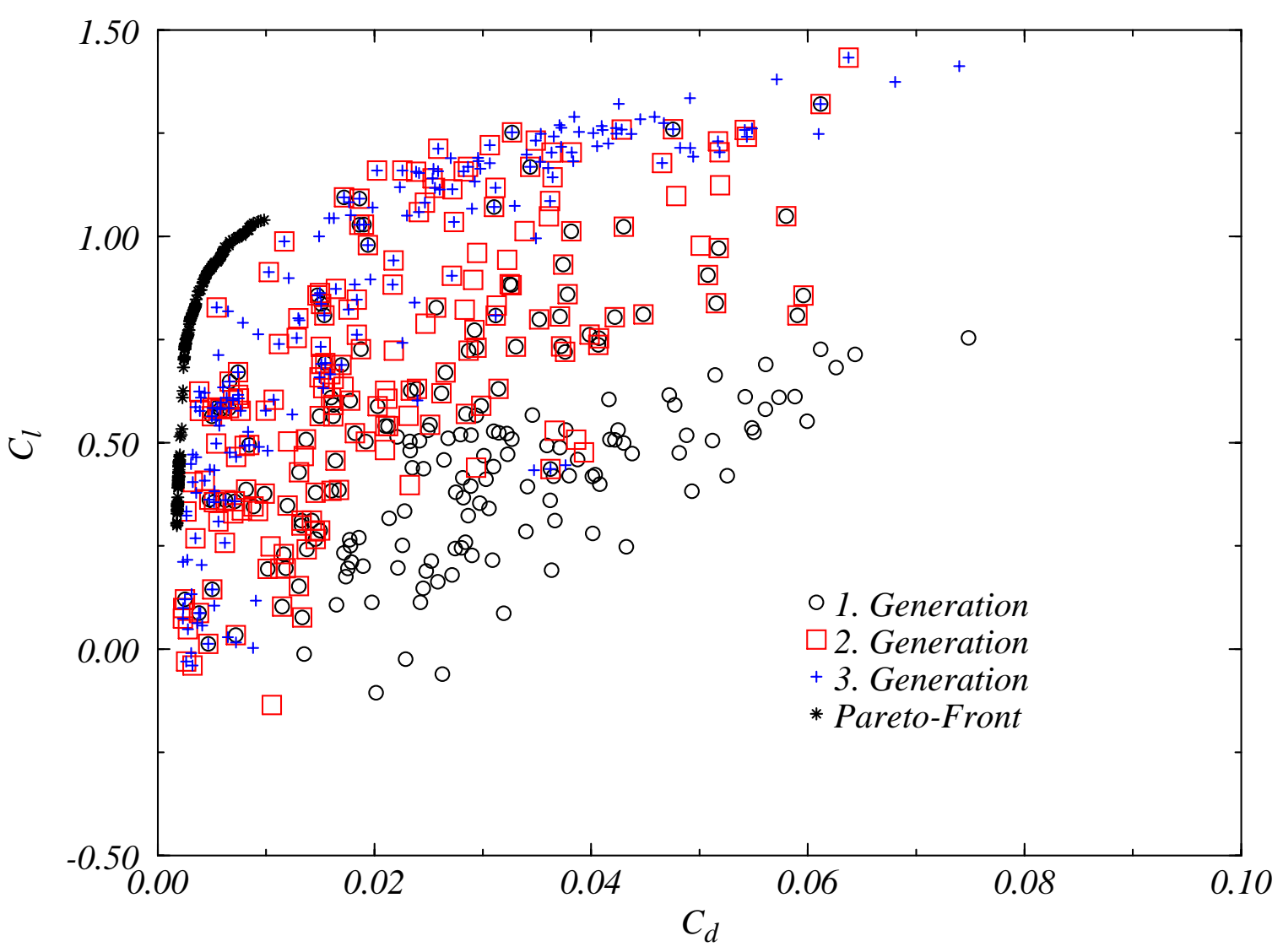

Abb. 12 Die Entwicklung zur Paretofront mit dem genetischen Algorithmus.

Wie aus der Grafik zu entnehmen ist, liegt die zweite Generation im Mittel schon besser als die erste Generation. Die zweite Generation liegt in der Grafik deutlich oberhalb der ersten Generation und wird von dieser großenteils durchdrungen. Die $C_{b}, C_{d}$-Werte der Profile der zweiten Generation liegen nicht alle besser als die der ersten. Sie liegen nur statistisch besser, weil die zweite Generation aus der besseren Hälfte der ersten Generation gebildet worden ist.

Bildet man nach demselben Verfahren die dritte Generation (blaue Kreuze) aus der zweiten Generation, sieht man denselben Effekt der Verbesserung wie bei der zweiten Generation gegen- 
über der ersten Generation. Tatsächlich sind wenige Profile der ersten Generation ebenfalls durch ein blaues Kreuz der dritten Generation gekennzeichnet. Das bedeutet, daß diese Profile durch ihre guten $C_{b}, C_{d}$-Werte bis in die dritte Generation überlebt haben.

Den Endzustand dieser Entwicklung stellt die Paretofront dar (schwarze Sterne), die quasi die Einhüllende der besten Profile von allen (meist der späten bis letzten) Generationen bildet. Die Paretofront wird hier nach ca. 50 Generationen erreicht. Die Paretofront stellt die Konvergenzlinie des GA bei der Mehrfachoptimierung dar. Durch den GA kann diese Front nicht überschritten werden, die Profile auf der Paretofront sind die besten, die durch so eine Vorgehensweise erreicht werden können.

Um die Paretofront gut aufzulösen, sind Profile mit einem Widerstandbeiswert von $C_{d}>0.01$ ausgeblendet worden. Die Profile in diesem Widerstandsbereich haben keine praktische Bedeutung. Für das Ausblenden dieses Widerstandsbereichs ist eine speziell angepaßte Operation erforderlich. Der GA selbst folgt nur der algorithmischen Vorschrift und blendet a priori keine Werte aus.

Tatsächlich ist eine gute Auflösung der Paretofront problematisch. In Abb. 12 sind in der Paretofront leichte Lücken zu erkennen. Diese Lücken sind unerwünscht. Ausnahmsweise haben die in diesem Beispiel aufgetretenen Lücken nichts mit einer schlechten Auflösung der Paretofront zu tun, denn auch andere Effekte kommen hier mit ins Spiel. Aber die Anzahl der Objekte, die die Paretofront schließlich bilden werden, ist nicht a priori festgelegt. Wählt man diese Anzahl zu gering, kann man nicht von einem optimalen Ergebnis ausgehen, denn es kommt dann zu einer vorzeitigen Konvergenz. Da eine korrekte Anzahl von Objekten aber von fundamentaler Bedeutung ist, muß diese Anzahl bestimmt werden, bevor man überhaupt von einer Paretofront sprechen kann. Deswegen wähle ich für diese Aufgabe eine einfache Geometriekonfiguration und versuche die minimal erforderliche Anzahl von Objekten zu bestimmen.

\subsection{Anwendung des Genetischen Algorithmus auf einsektionale Profile}

Die einfachste Möglichkeit der Geometriedefinition nach der oben beschriebenen Vorgehensweise für eine einfache Konfiguration ist ein einsektionales Profil. Da es zunächst nicht um das Ausschöpfen aller geometrischer Möglichkeiten zur aerodynamischen Leistungssteigerung eines Profils geht sondern darum, eine Größe für die Qualität der Paretofront zu bestimmen, ist für einen ersten Probelauf die einfache Geometriedefinition sinnvoll.

In einem ersten Schritt muß dazu die minimale Anzahl der Objekte bestimmt werden, die für eine Konvergenz des GA gegen die Paretofront notwendig ist. Es liegt auf der Hand, daß eine zu klein gewählte Anzahl nicht gegen die letztendlich optimale Paretofront konvergiert. Um diesen Sachverhalt zu verdeutlichen, stelle man sich eine Anzahl (Population) von zwei Objekten zur Optimierung vor. Wo auch immer die aerodynamischen Beiwerte $\mathrm{C}_{1}$ und $\mathrm{C}_{\mathrm{d}}$ dieser Konfigurationen liegen werden, sie bilden nach der Definition (8) eine Paretofront, wenn keines der beiden Objekte innerhalb des optimalen Quadranten des anderen liegt. Es ist klar, daß damit nichts erreicht ist. Erst eine adäquate Anzahl von Objekten, die alle auf der Paretofront liegen, können eine sinnvolle Aussage über die aerodynamische Leistungsfähigkeit der Konfiguration und seiner geometrischen Definition, machen. 
In der Literatur findet sich kein Hinweis darüber, wie groß diese Anzahl mindestens sein muß. Diese Anzahl ist offensichtlich so problemspezifisch, daß im Vorfeld einer Optimierung diese Anzahl bestimmt werden muß. Natürlich wird man bestrebt sein, die kleinste notwendige Anzahl von Objekten zu wählen, denn je größer diese Anzahl ist, umso mehr ist auch zu berechnen. Einer sicheres Indiz für das Erreichen dieser unteren Grenze ist die Tatsache, daß sich bei einer Vergrößerung der Anzahl die Lage der Paretofront nicht mehr ändert. Aus diesem Grund versuche ich anhand eines einfachen Beispiels eine untere Grenze für die erforderliche Anzahl von Objekten zu finden.

Auf die bei GA übliche binäre Codierung wird hier zugunsten einer numerischen (reellen) Codierung verzichtet, siehe (2). Die numerische Codierung erhält aufgrund der besseren Handhabung den Vorzug. Für den genetischen Algorithmus ist die Vorgehensweise, in Abb. 3 dargestellt, zu wählen.

Die vollständige Definition einer Kurve in der $(\varphi, r)$-Ebene enthält Parameter, die bei Wahl einer einzigen Sektion (einer Kurvendefinition über das Intervall $[0,2 \pi]$ ) für das komplette Profil als variable Parameter benötigt werden, für den GA jedoch zunächst nicht benötigt werden. Diese sind aber bei Verwendung mehrerer Sektionen zu berücksichtigen und dann als weitere Variable in den genetischen Vektor einzufügen. Der vollständige Parametersatz für eine Kurvendefinition in $\operatorname{der}(\varphi, r)$-Ebene lautet:

Tabelle 1 Parametersatz einer Sektion

\begin{tabular}{|c|l|c|}
\hline Parameter & \multicolumn{1}{|c|}{ Beschreibung } & als genetischer Parameter \\
\hline \hline key & allgemeine Kennzahl & fest \\
\hline $\mathrm{g}$ & Kennzahl Kurvenfunktion & fest \\
\hline$\varphi_{1}$ & Anfangspunkt $\varphi, r-E b e n e$ & fest \\
\hline $\mathrm{r}_{1}$ & Anfangspunkt $\varphi, r$-Ebene & variabel \\
\hline $\mathrm{s}_{1}$ & funktionsabhängiger Parameter & variabel \\
\hline $\mathrm{s}_{2}$ & funktionsabhängiger Parameter & variabel \\
\hline $\mathrm{e}_{1}$ & funktionsabhängiger Parameter & variabel \\
\hline $\mathrm{e}_{2}$ & funktionsabhängiger Parameter & fest \\
\hline$\varphi_{2}$ & Endpunkt $\varphi, r-E b e n e$ & fest \\
\hline $\mathrm{r}_{2}$ & Endpunkt $\varphi, r-E b e n e$ & \\
\hline
\end{tabular}

Der genetische Vektor hat dann die Form wie in (2). Aus der Tabelle 1 ist zu ersehen, daß eine Optimierung für zunächst nur eine Sektion anhand von vier Variablen durchzuführen ist, wenn man Anfangs- und Endpunkt der Sektion festhält. Das entspricht einer Optimierung im Hyperraum der Dimension 4. Ob alle variablen Parameter optimiert werden müssen, hängt von der Wahl der Funktion ab. Ist die gewählte Funktion von nur zwei Parametern abhängig, entspricht 
die Optimierung einer Suche des Maximums einer zweidimensionalen Funktion. Das Beispiel bildet hier den einfachsten Fall. Bewußt wird hier von Anfang an die umfassendere Vorgehensweise gewählt, da komplexere Konfigurationen in höherdimensionalen Räumen definiert sind und sich somit die Zahl der zu optimierenden Parameter schnell erhöht.

Auf die so definierte Geometrie wird der GA angewendet. Aufgetragen werden, wie in Abb. 12 dargestellt, die Auftriebsbeiwerte über den Widerstandsbeiwerten der letzten Generation (denn das ist die Paretofront). Es wurden mehrere Rechnungen mit verschiedener Anzahl von Objekten durchgeführt. Hier stelle ich die Rechnung mit 200 Objekten in Abb. 13 einer Rechnung mit 400 Objekten in Abb. 14 gegenüber. Die Strömungsdaten wie Machzahl und Anstellwinkel sind beibehalten.

Zunächst ist festzustellen, daß die beiden Paretofronten nahezu denselben Verlauf haben. Ein Unterschied besteht in der unterschiedlich dichten Punktebesetzung der Fronten, bedingt durch die unterschiedliche Anzahl von Punkten. Dennoch sind beide Kurven, abgesehen von Bereichen schwacher Besetzung, identisch. In Bereichen schwacher Besetzung haben die Paretofronten einen weniger glatten Verlauf. Die kleinen Sprünge, die hier sichtbar sind, sind statistische Abweichungen von der Paretofront, die natürlich um so kleiner werden, je mehr Punkte in dem entsprechenden Bereich vorhanden sind. Es ist zwar möglich, bestimmte Bereiche durch verschiedene genetische Evaluationstechniken statistisch besser zu besetzen, aber die Besetzung über die gesamte Kurve gleichmäßig zu verteilen ist nur durch ein sehr aufwendiges Studium der Parameter des Fitneß-Sharing zu realisieren. Das ist sinnvoll, wenn man ein Profil für einen bestimmten Designpunkt $\left(\mathrm{C}_{\mathrm{l}}, \mathrm{C}_{\mathrm{d}}\right.$ gegeben) entwerfen möchte. Beispielsweise ist das aerodynamische Optimum ein solcher Bereich der Kurve, um dessen Zentrum ein gute Besetzung der Paretofront erwünscht ist.

Die Paretofronten, die mit einer Anzahl von 50-100 Objekten gerechnet wurden, liegen schlechter als die Front mit 200 Objekten, und sind hier nicht dargestellt. Es ist daher geboten die Rechnungen für die Paretofronten mit mindestens 200 Objekten durchzuführen. Tatsächlich wird dieses eine konstante Anzahl von Objekten zukünftiger Rechnungen sein.

Ein weiteres wichtiges Ergebnis, welches aus der Lage der Paretofronten folgt, ist die Unabhängigkeit des Ergebnisses von den Anfangsbedingungen. Bei der genetischen Initialisierung werden die Parameter der Funktionen zufällig aber in gewissen Grenzen gewählt. Die Entwicklung der Population (der Menge von Objekten) mit 200 Individuen (Objekten) zeigt genau den gleichen Endzustand wie die Rechnung mit 400 Individuen. Der Anfangszustand dieser beiden Rechnungen muß allein schon wegen der unterschiedlichen Anzahl der Objekte unterschiedlich gewesen sein. Das Endergebnis (die Lage der Paretofront) ist in beiden Fällen aber gleich. Diese Tatsache läßt den logischen Schluß zu, daß der Anfangszustand beliebig sein kann. Der genetische Algorithmus konvergiert in jedem Fall gegen die gleiche Paretofront, wenn man mindestens mit 200 Individuen für dieses Problem rechnet.

Aus den hier aufgeführten Ergebnissen kann darauf geschlossen werden, daß die oben erwähnte minimale untere Grenze für die Anzahl der Objekte, recht gut bestimmt ist. Ich habe vorne darauf hingewiesen, daß bei dem Verfahren, den Fitneßwert für jedes Profil/Tragflügel über eine Eulerrechnung zu bestimmen, aus Zeitgründen nur die maximal notwendige Anzahl von Objekten gewählt werden sollte. Die hier gefundene Anzahl bringt dasselbe Ergebnis wie jede höhere Anzahl von Objekten (abgesehen von der Gleichmäßigkeit der Besetzung). Deswegen nehme 
ich den hier gefunden Wert als diese untere Grenze an und verwende ihn in zukünftigen Rechnungen.

\section{Paretofront}

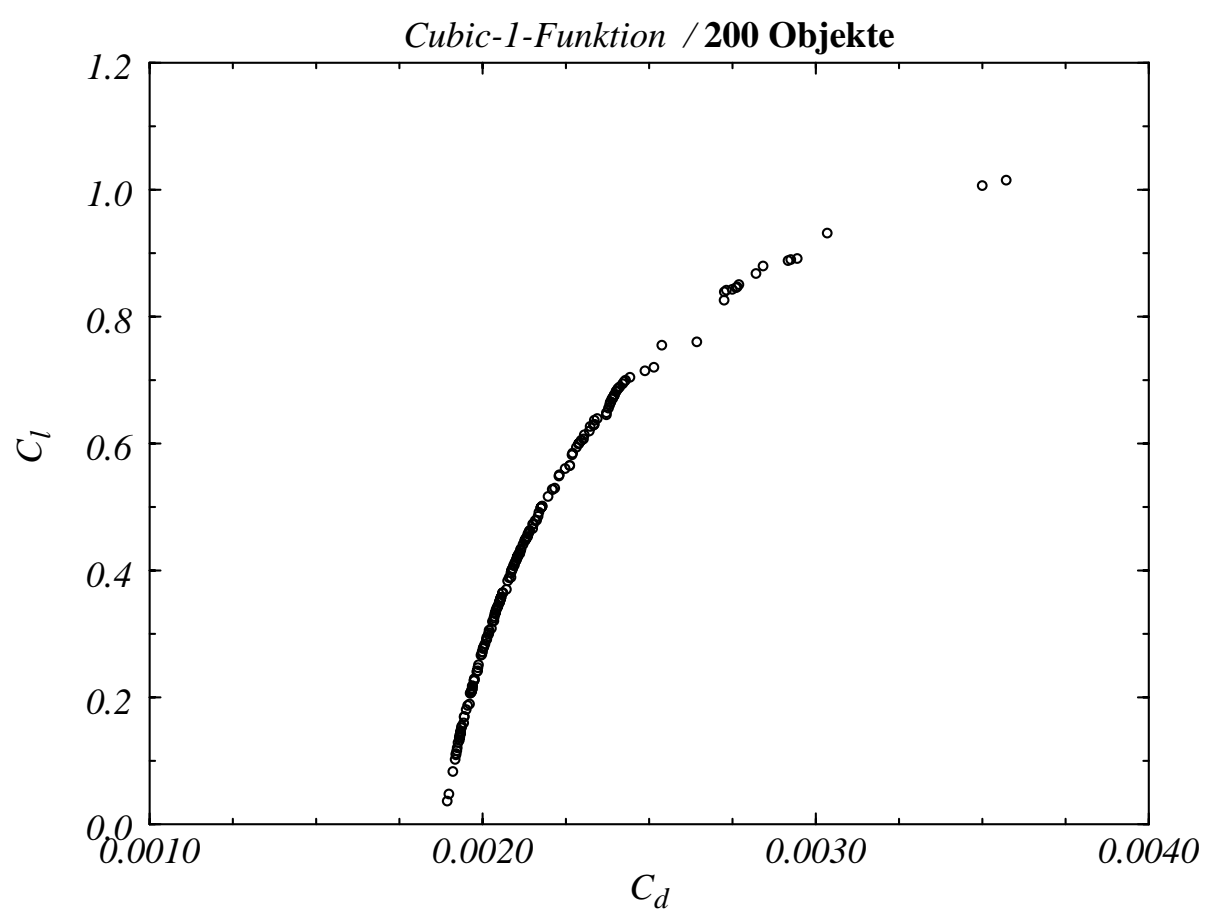

Abb. 13 Paretofront der Cubic-Funktion, eine Sektion, $M_{\infty}=0.707, \alpha=1.46^{\circ}, 200$ Punkte.

\section{Paretofront}

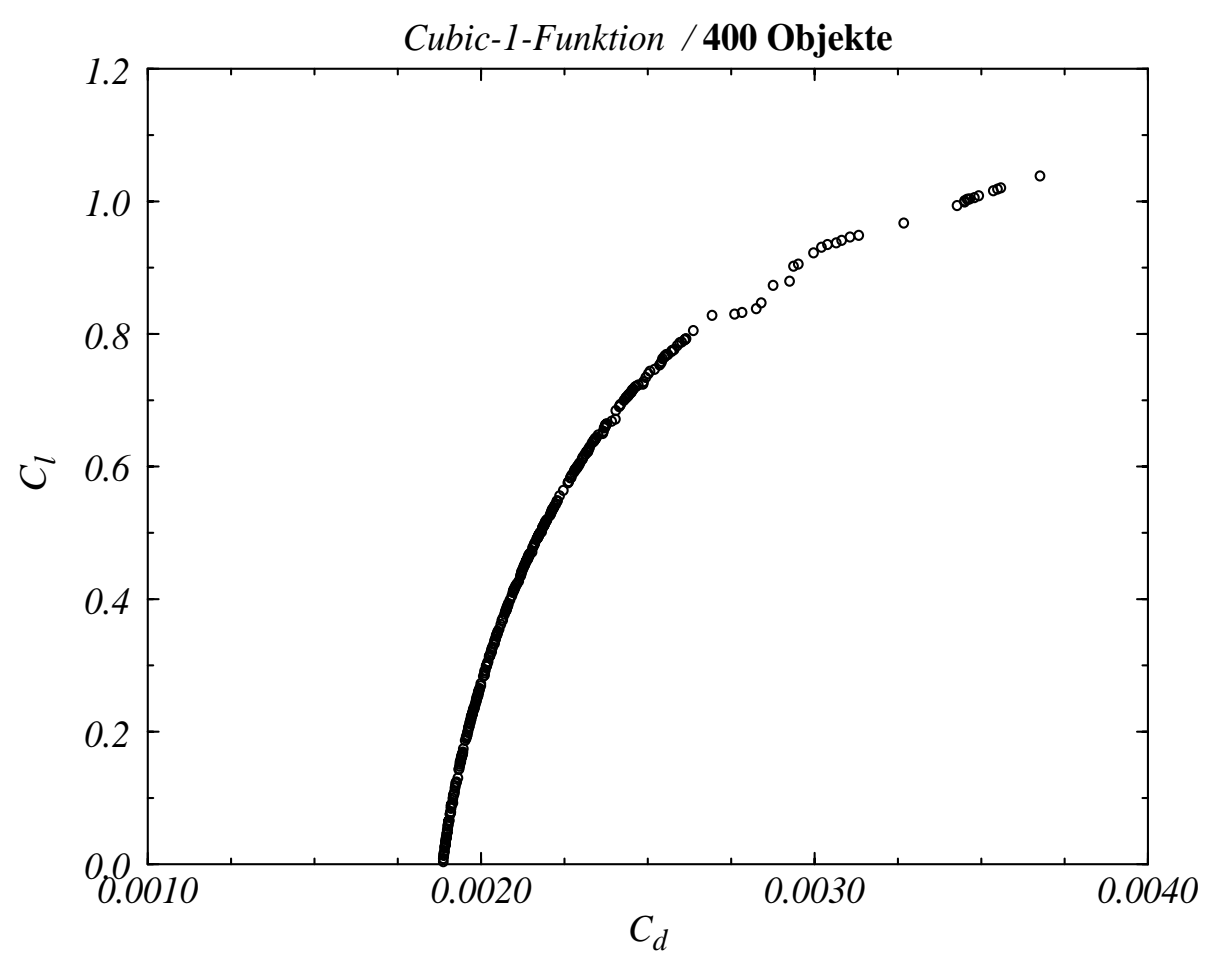

Abb. 14 Paretofront der Cubic-Funktion, eine Sektion, $M_{\infty}=0.707, \alpha=1.46^{\circ}, 400$ Punkte. 


\subsection{Erweiterung auf mehrsektionale Profile verschiedener Funktionen}

Mit Hilfe der einsektionalen Profile ist die untere Grenze der Populationsgröße festgestellt worden. Die daraus resultierende Paretofront stellt in dem Rahmen der Geometriedefinition und der angegebenen Parameter das rechentechnisch maximal zu erreichende Ergebnis dar. Durch den implementierten Algorithmus kann in diesem Parameterbereich keine weitere Verbesserung vorgenommen werden. Jeder Punkt der Paretofront repräsentiert jeweils ein Profil. Jedes Profil ist optimal innerhalb der gewählten Geometriedefinition und den vorgegebenen Parametergrenzen. Die Grenzen des Funktionsparameterbereichs sind so gewählt, daß die Parameter der optimalen Profile auf der Paretofront deutlich innerhalb dieser Grenzen liegen. Das wird in folgenden Kapiteln noch deutlich werden. Es stellt sich die Frage, mit welchen Mitteln diese Paretofronten zu überbieten sind. Die einsektionale Geometriedefinition ist offensichtlich voll ausgereizt.

Es ist zu erwarten, daß bei Hinzufügen zusätzlicher Gestaltungsmöglichkeiten der Geometrie, ein weiter verbessertes Ergebnis erzielt werden kann. Diese zusätzlichen Gestaltungsmöglichkeiten könnte man durch eine weitere Unterteilung des Kurvenverlaufs in der $\varphi$,r-Ebene in mehrere Sektionen erreichen. Anstelle einer Sektion könnte man zwei oder mehr Sektionen definieren (siehe Abb. 6). Damit wären die Gestaltungsmöglichkeiten erheblich erweitert, und der Algorithmus sollte damit in der Lage sein die Profilkontur weiter zu verbessern. Damit sollte dann auch eine überlegene Paretofront, gegenüber der oben aufgetragenen Paretofront für einsektionale Profile, möglich sein. Ob das tatsächlich der Fall sein wird und in welchen Bereichen dies zutrifft, muß erst eruiert werden.

Eine andere Möglichkeit eine verbesserte Paretofront zu erhalten, wäre die oben verwendete Cubic-Funktion durch eine flexiblere Funktion zu ersetzen. Die Cubic-Funktion hat nur zwei abhängige Parameter (siehe Anhang). Es wäre interessant, diese Funktion gegen eine andere Funktion zu tauschen, und zu überprüfen, ob die durch die zusätzlichen Parameter erweiterte Geometriedefinition sich in der Lage der Paretofront niederschlägt. Auch dieser Sachverhalt wird Gegenstand weiterer Untersuchungen sein.

\section{Cubic-Funktion mit zwei Sektionen}

Als ersten Testfall erweitere ich die Geometriedefinition um eine Sektion auf zwei Sektionen. Die erste Sektion in der $\varphi, r-E b e n e$ erstreckt sich über das Intervall $[0, \pi]$. Daran schließt sich nahtlos das Intervall $[\pi, 2 \pi]$ an. Siehe dazu Abb. 6. Diese Unterteilung in der $\varphi, r-E b e n e$ führt zu einer Unterteilung des Profils in Ober- und Unterseite, mit der Nahtstelle im Staupunkt. Diese Wahl der Nahtstelle bedeutet hier eine höhere Flexibilität im Nasenbereich des Profils. Da mit der Nutzung von zwei Sektionen (jeweils Profilober- und Unterseite) für die Profildefinition eine größere Auswahl an geometrischen Möglichkeiten besteht, würde ich erwarten, daß hier bessere Profile als durch Definition durch eine Sektion entstehen. Damit sollte dann die berechnete Paretofront im $\mathrm{C}_{\mathrm{d}}, \mathrm{C}_{\mathrm{l}}$-Diagramm weiter links und oberhalb der Paretofront einsektionaler Profile liegen. Insgesamt sollten durch die Definition der Cubic-2-Funktion (Cubic-Funktion mit 2 Sektionen) die Profile einen höheren Leistungsbereich erschließen. 


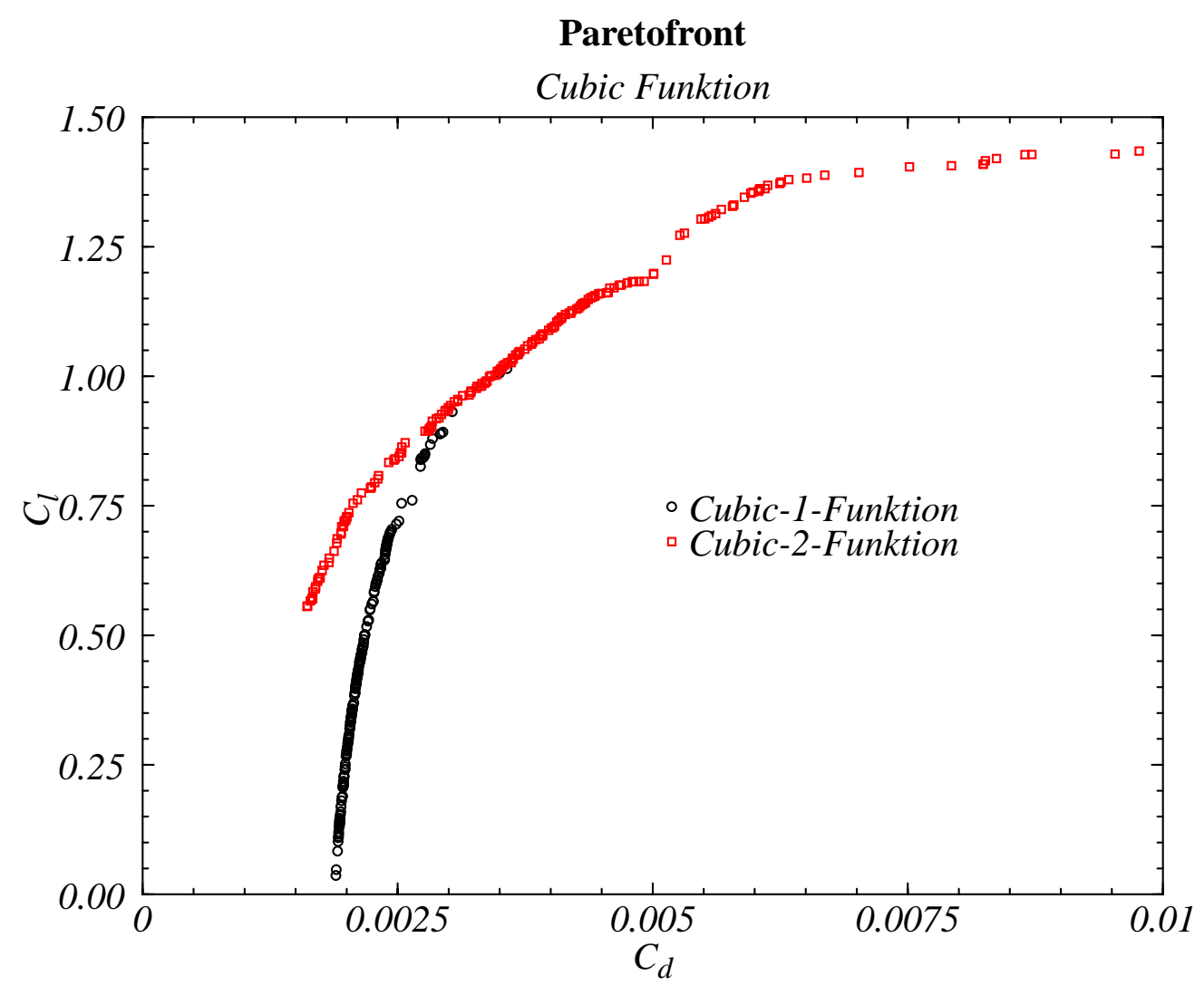

Abb. 15 Paretofront der Cubic-Funktionen. $M_{\infty}=0.707, \alpha=1.46^{\circ}, 200$ Punkte.

Diese Abbildung zeigt die Paretofronten der Cubic-Funktion mit einer bzw. zwei Sektionen. Der hier sichtbare Verlauf der Paretofronten entspricht den oben genannten Erwartungen. Durch die zusätzlichen Möglichkeiten der Geometriedefinition durch zwei Sektionen entsteht auch eine verbesserte Paretofront. Diese liegt jenseits der Paretofront, die durch eine Sektion errechnet wurde.

Zunächst ist auffällig, daß die Paretofront der Cubic-2-Funktion einen sehr viel größeren Bereich abdeckt als die Front der Cubic-1-Funktion. Der Verlauf im unteren Bereich zeigt, daß dort leistungsfähigere Profile möglich sind als mit den beschränkteren Mitteln der Cubic-1Funktion. Die Paretofront der Cubic-2-Funktion erschließt vor allem einen höheren Bereich von $\mathrm{C}_{1}$ und $\mathrm{C}_{\mathrm{d}}$. Ganz offensichtlich sind durch die zusätzlichen geometrischen Gestaltungsmöglichkeiten Profilkonturen überhaupt erst möglich, die der Cubic-1-Funktion vollständig verschlossen waren. Aber auch die Paretofront der Cubic-2-Funktion scheint für hohe Auftriebs- und Widerstandsbeiwerte einem Grenzwert entgegenzustreben. Dafür spricht der nahezu horizontale Verlauf der Paretofront in diesem Bereich. Die geometrischen Mittel sind hier offensichtlich erschöpft. Die schwache Punktbesetzung spricht hier für einen ungenauen Verlauf der Paretofront. Dieser Effekt konnte schon in Abb. $13 \mathrm{im}$ Bereich $C_{l} \sim 0.8$ beobachtet werden. Durch die geringe Besetzung ist der Verlauf nicht ganz genau derselbe, wie bei es bei einer stärkeren Besetzung der Fall wäre. Der Verlauf wird aber qualitativ richtig wiedergegeben. 
Die Paretofront der Cubic-2-Funktion bricht unter $C_{l} \sim 0.55$ abrupt ab. Die Gründe hierfür sind zunächst nicht klar. Möglicherweise ist es zu früh, diesen Sachverhalt zu diskutieren. Dazu existieren an dieser Stelle noch zu wenig Erfahrungen mit den Paretofronten und dieser Art von Geometriedefinition. Sollte dieser Effekt bei zukünftigen Paretofronten auch auftreten, ist sicher eine bessere Interpretation möglich, weil dann mehr Ergebnisse zur Verfügung stehen.

Auf die Besonderheiten der Profile selbst werde ich erst später genauer eingehen. Zu diesem Zeitpunkt haben die Profile noch sehr akademischen Charakter. Das Ziel momentan ist, Erfahrungen über den Zusammenhang der Geometriedefinition und der damit verbundenen Leistungsfähigkeit der Objekte zu gewinnen. Diesen Zusammenhang gibt der Verlauf der Paretofront wieder.

\section{Cubic-Funktion mit vier Sektionen}

Die logische Erweiterung der zweisektionalen Profile sind die viersektionalen Profile. Hier tritt nun ein Besonderheit auf: Die Einteilung der Intervalle für vier Sektionen ergibt sich nicht aufgrund neuralgischer Punkte am Profil. Für zweisektionale Profile ist es naheliegend, die Nahtstellen der Sektionen in die obere Hinterkante, in den Nasenpunkt und in die untere Hinterkante zu legen. Damit teilt man das Profil in Ober- und Unterseite ein. Das ist sinnvoll, da die Profilkontur von Ober- und Unterseite nur wenig voneinander abhängen. Zwar existiert, strömungsmechanisch gesehen, für elliptische Probleme im Unterschall eine starke Wechselwirkung, aber keine direkte Abhängigkeit des Konturverlaufs. Anders ist es, wenn man die Ober- oder Unterseite des Profils selbst in zwei Bereiche aufteilt. Dann teilt man das Profil zusätzlich in einen vorderen und hinteren Bereich auf. Der gegenseitige Abhängigkeit der Kontur an der Oberfläche im vorderen und hinteren Bereich ist sehr viel stärker, als im oberen und unteren Bereich. Hierfür geben viele transsonische Phänomene ein Beispiel.

Bei vier Sektionen müssen zwei weitere Punkte $(\varphi, r)$ bestimmt werden, die die Nahtstelle von vorderem und hinterem Profilbereich bilden. Da kein Punkt für diese Nahtstelle ausgezeichnet ist, wähle ich diesen Punkt in der $\varphi, r$-Ebene auf der Oberfläche bei $\pi$, auf der Unterseite bei $3 / 2 \pi$ (siehe Abb. 6). Bei sämtlichen Rechnungen lasse ich diesen Punkt zunächst fest. Ob auch dieser Punkt Gegenstand genetischer Optimierung sein sollte, ist später zu entscheiden. Prinzipiell ist zu vermuten, daß zusätzliche geometrische Möglichkeiten in einen höheren Leistungsbereich eines Profils führen. Wie man im folgenden noch sehen wird, ist dieser Schluß nicht für alle Parameter zwingend.

Die Möglichkeit der Geometriedefinition, die sich aus der Wahl genau dieses Punktes ergibt, wird im folgenden noch von Vorteil sein. Denn durch diese Wahl wird sich die prozentuale Profildicke recht bequem vorgeben lassen. Wird also zusätzlich an den Punkten $(\pi, r)$ und $(3 / 2 \pi, r)$ das $r$ konstant gehalten, so sind diese Punkte Fixpunkte der Kontur. In der abgebildeten Ebene sind damit ebenfalls Fixpunkte definiert. Eine prozentuale Dicke definiere ich über den Abstand dieser Punkte in der abgebildeten Ebene. 


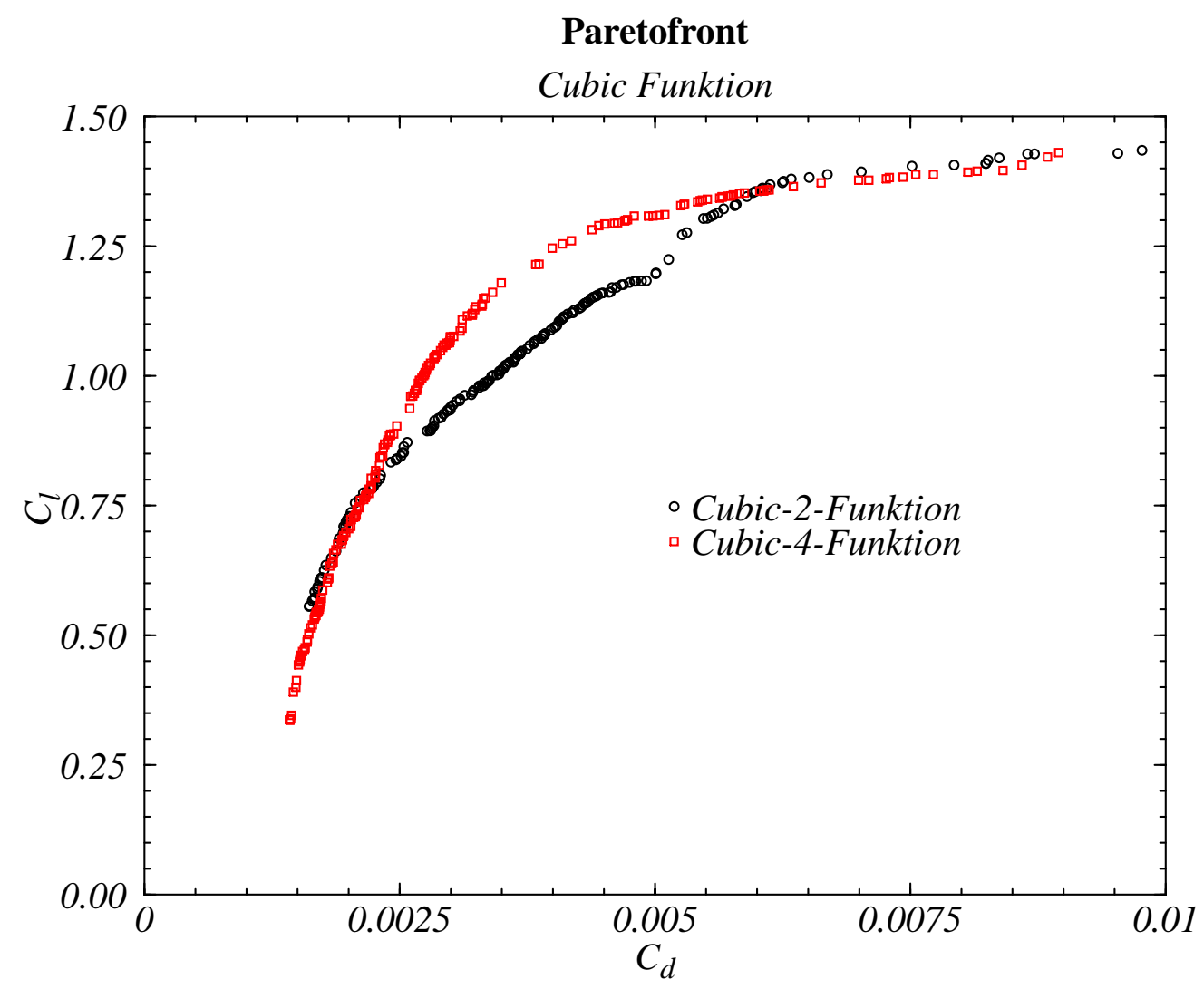

Abb. 16 Paretofront der Cubic-Funktionen. $M_{\infty}=0.707, \alpha=1.46^{\circ}$.

Diese Abbildung zeigt den Vergleich der Cubic-Funktion für zwei und vier Sektionen. Das $r$ der

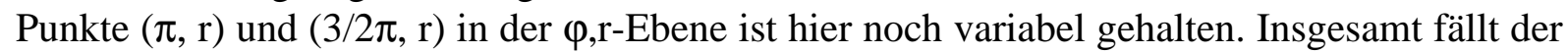
Vergleich hier wie schon bei der Gegenüberstellung von ein- und zweisektionalen Profilen aus. Die Paretofront der Cubic-4-Funktion liegt besser als die Paretofront der Cubic-2-Funktion, mit einigen wichtigen Unterschieden. Insbesondere die Bereiche hoher und kleiner Werte von $\mathrm{C}_{1}$ und $\mathrm{C}_{\mathrm{d}}$ der Paretofronten zeigen nur eine kleine Differenz. Die Kurven liegen im Bereich $C_{l}<0.8$ und $C_{d}>0.006$ nahezu übereinander. Der Bereich zwischen diesen Werten stützt wieder die These, daß erweiterte Möglichkeiten der Geometriedefinition auch leistungsfähigere Profile produzieren. Zumindest zwischen den beiden zuletzt genannten Werten ist das auch der Fall, denn die Paretofronten klaffen hier deutlich auseinander.

Einigermaßen überraschend ist die Tatsache, daß die Überlegenheit der Cubic-4-Funktion nur in einem bestimmten Bereich zutage tritt. In den Bereichen in Abb. 16, in denen die Fronten übereinanderliegen, sind die Profile der Cubic-2-Funktion denen der Cubic-4-Funktion aerodynamisch gleichwertig. Insbesondere der Bereich hoher Widerstandsbeiwerte, wo hohe geometrische Flexibilität gefordert wird, bringt die Cubic-4-Funktion keinen Vorteil. Dieses Resultat ist nicht sofort verständlich.

Die Vermutung, daß erweiterte Möglichkeiten der Geometriedefinition auch leistungsfähigere Profile reproduzieren, bestätigt sich zwar, aber Profile mit unterschiedlich vielen Sektionen gehören im Grunde nicht derselben Kategorie an. Denn die Cubic-4-Funktion hat hier den Nach- 
teil, daß die Kurve der $\varphi$,r-Ebene durch zwei weitere Punkte laufen muß. Die Randbedingung muß die Cubic-2-Funktion nicht erfüllen. Wie oben erwähnt, erlauben die zusätzlichen festgehaltenen Punkte eine gute Fixierung der Profildicke. Es ist zu vermuten, daß für $C_{l}<0.8$ und für $C_{l}>1.35$ aus den Cubic-2-Funktionen dünnere optimale Profile gefunden werden, als sie bei der Fixierung mittels Cubic-4 entstehen. Dünnere Profile haben aber in der Regel geringeren Widerstand im Transsonikbereich. Weiter spielt aber die gewählte Funktion auch eine Rolle. Um diesen Sachverhalt weiter zu untersuchen, tausche ich die Funktion selbst aus, und wiederhole die Rechnungen unter den oben genannten Randbedingungen.

\section{Quintic-Funktion mit zwei Sektionen}

Im Unterschied zur Cubic-Funktion ist die Quintic-Funktion von zwei weiteren Parametern abhängig. Diese zwei weiteren Parameter ermöglichen es, neben den Tangenten auch die zweiten Ableitungen am Anfangs- und Endpunkt einer Sektion vorzugeben. Somit ist auch bei gleicher Anzahl von Sektionen eine Überlegenheit der Quintic-Funktion gegenüber der Cubic-Funktion als Grundlage einer Profildefinition zu erwarten. Ob sich diese Vermutung bestätigen läßt, zeigen die folgenden Abbildungen.

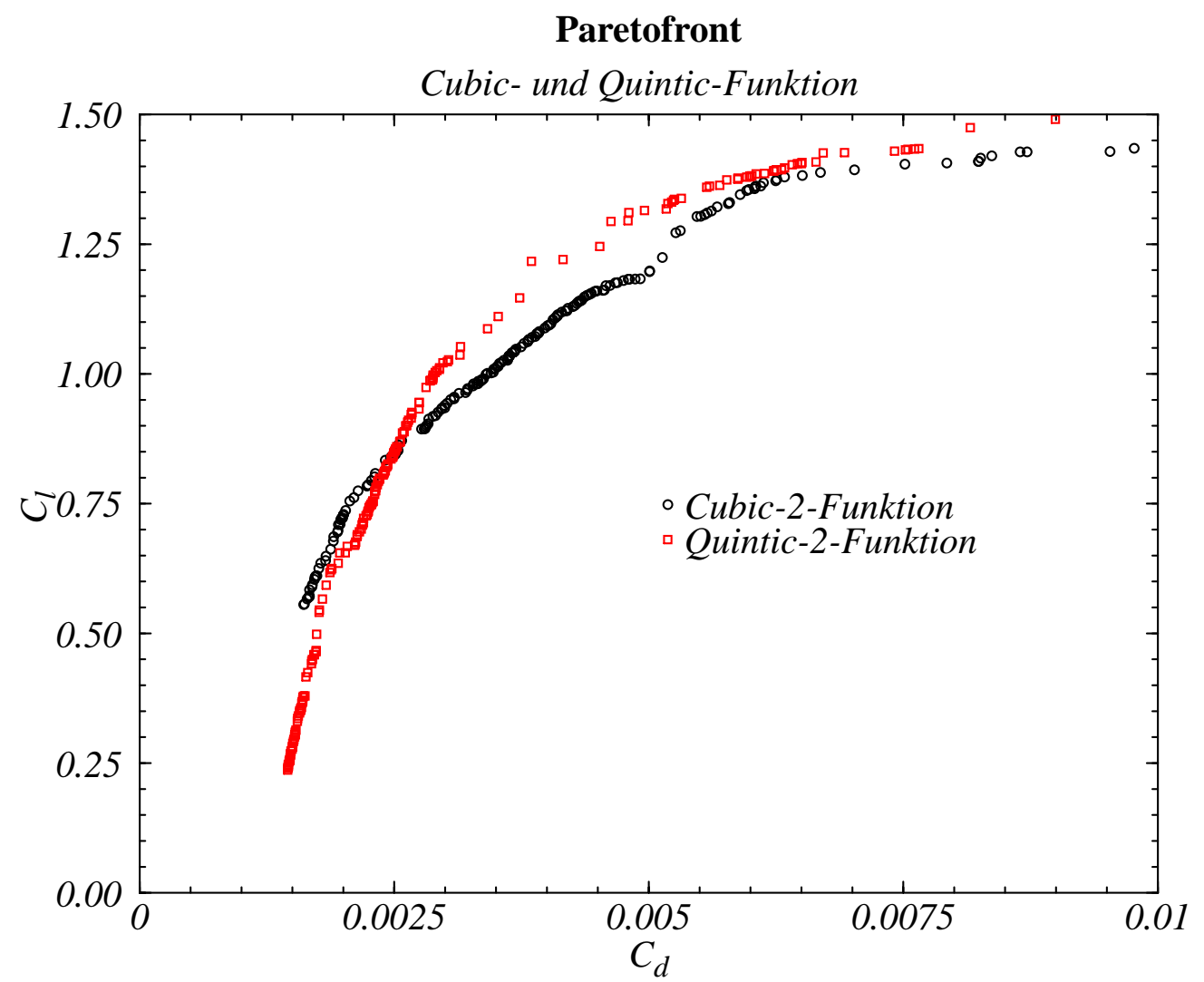

Abb. 17 Paretofront der Cubic- und Quintic-Funktionen. $M_{\infty}=0.707, \alpha=1.46^{\circ}$.

In Abb. 17 ist der Vergleich der Cubic- und Quintic-Funktion mit zwei Sektionen gezeigt. Die Darstellung ähnelt stark der Abb. 16, in der der Vergleich der Cubic-2- und Cubic-4-Funktion 
vollzogen wird. Auch in diesem Fall bestätigt sich, daß eine Erweiterung der geometrischen Definition durch zusätzliche Parameter zu einer Verbesserung der aerodynamischen Leistungsfähigkeit der Profile führt. Für Werte $C_{l}>0.8$ in Abb. 17 ist dieser Sachverhalt gegeben. Diesmal wurde die Erweiterung der geometrischen Definition anstelle weiterer Sektionen durch weitere Funktionsparameter realisiert. Die Paretofront der Quintic-2-Funktion verläuft stets oberhalb der Paretofront der Cubic-2-Funktion. Im dem Bereich $C_{l}<0.8$ drehen sich die Verhältnisse um. In diesem Bereich liefert die Cubic-2-Funktion bessere Profile. Dieses Ergebnis wurde weiter oben im Zusammenhang der Cubic-4-Funktion schon diskutiert. Der Schluß von der Erweiterung der geometrischen Definition durch zusätzliche Parameter auf verbesserte Profile ist in großen Grenzen zulässig, aber nicht überall zwingend.

Abschließend sei noch der Vergleich der Cubic-4-Funktion und Quintic-4-Funktion vorgestellt. Die Paretofronten dieser beiden Funktionen sind in Abb. $18 \mathrm{zu}$ sehen. Die Diskussion dieser Kurven unterscheidet sich nicht wesentlich von der oben geführten Diskussion. Bemerkenswert ist aber doch der ausgedehnte Bereich, über den die Cubic-4-Funktion der Vorzug gegenüber der Quintic-4-Funktion genießt. Erst bei $C_{l} \sim 1.25$ kehrt sich dieser Vorzug um. Demnach kristallisiert sich (ohne eine detaillierte Untersuchung) die Gesetzmäßigkeit heraus, daß zumindest bei hohen Auftriebsbeiwerten eine höhere Vielfalt geometrischer Entwurfsmöglichkeiten zur Verfügung stehen muß, um gute Ergebnisse hinsichtlich der aerodynamischen Leistungsfähigkeit zu erzielen.

\section{Paretofront}

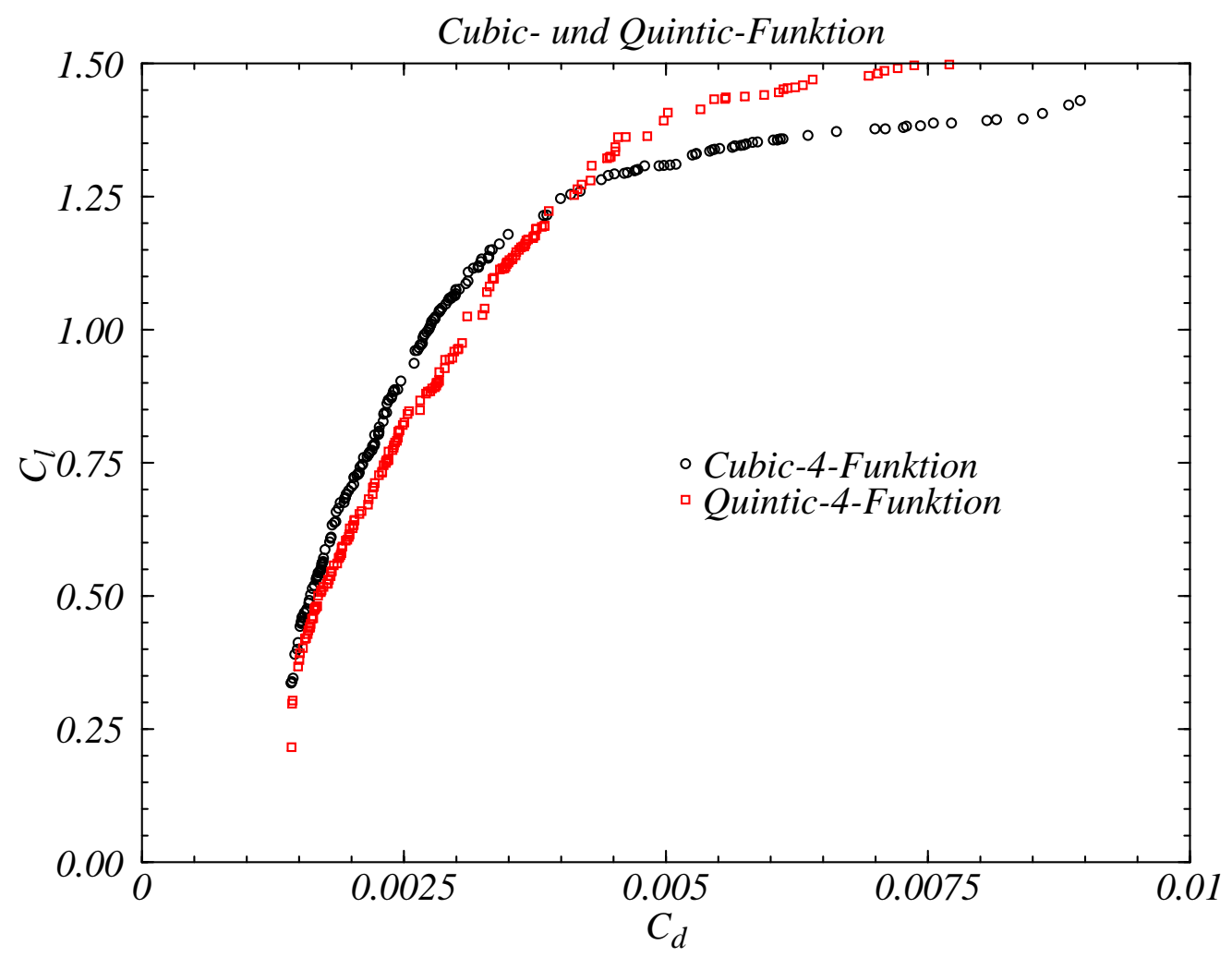

Abb. 18 Paretofront der Cubic-4 und Quintic-4-Funktionen. $M_{\infty}=0.707, \alpha=1.46^{\circ}$.

Prinzipiell ist der Unterteilung der Profile in Kurvenabschnitte durch Hinzufügen weiterer Sek- 
tionen keine Grenze gesetzt. Man kann an den bisher gemachten Erkenntnisse jedoch ablesen, daß die Vorgehensweise nicht zwingend zum erwünschten Erfolg führen muß. Für kleine Abschnitte auf der Paretofront schneiden teilweise geometrisch eingeschränktere Profile aerodynamisch besser ab. Bevor man also der Geometriedefinition weitere Gestaltungsmöglichkeiten eröffnet, sollte der Bereich festgelegt werden, für den man einen Entwurf durchführen will. Es zeigt sich hier, daß allgemeine Gesetzmäßigkeiten nur mittels weitergehender Diskussion des resultierenden Strömungsfeldes abzuleiten sind. Dennoch lassen sich einige Erkenntnisse aus den durchgeführten Rechnungen ableiten, die in den folgenden Kapiteln noch von Bedeutung sein werden.

\section{Zusammenfassung}

In einer ersten Übersicht fasse ich Gesetzmäßigkeiten zusammen, die sich aus den oben gewonnenen Erkenntnissen ergeben.

- Die Lage der Paretofront ist:

•• unabhängig von der Anzahl der berechneten Objekte, wenn die Anzahl ,nicht zu klein ist“".

Wie oben durchgeführt, muß die minimale Anzahl zu berechnender Objekte für jede Problemstellung separat neu durchgeführt werden. Eine zu kleine Anzahl der Objekte führt zu einer minderwertigen Paretofront.

•• abhängig vom Strömungslöser selbst.

Die Unterschiede bestehen aufgrund der verschiedenen Verfahrensweisen von z.B. Panel-Code, Euler-, Navier-Stokes-Verfahren. In den Verfahren sind unterschiedliche physikalische Phänomene berücksichtigt. Deswegen ist die Gültigkeit der Paretofront nur innerhalb desselben Verfahrens schlüssig.

•• abhängig von der Geometriedefinition.

Die Geometriedefinition spielt eine große Rolle. Insbesondere sollte der Rahmen der geometrischen Möglichkeiten weit gesteckt sein, wenn aerodynamische Entwürfe im Hochauftriebsbereich erwünscht sind. Im niederen Auftriebsbereich führen einfache Konfigurationen schon zu guten Ergebnissen. Eine Erhöhung der Anzahl der Sektionen bringt aerodynamisch gesehen keine Vorteile im unteren Bereich von $C_{1}$ und $C_{d}$.

•• abhängig von den Strömungskennzahlen.

Die Strömungskennzahlen wie Machzahl und Anstellwinkel geben natürlich andere Randbedingungen in dem Strömungslöser vor und führen damit zu anderen Paretofronten.

- die Paretofront ist um so glatter, je mehr Objekte berechnet werden,

Dieser Sachverhalt ergibt sich aus statistischen Betrachtungen. Gewisse Unstetigkeiten auf der Paretofront sind nur durch Erhöhung der Anzahl der Objekte zu beheben. Die Qualität der Paretofront wird dadurch nicht wesentlich verbessert. 
- die Anzahl der Parameter erhöht nicht notwendig die Anzahl der zu berechnenden Objekte.

Dieses ist eines der wichtigsten und angenehmsten Ergebnisse bezüglich der EM. Denn in den oben ausgeführten Betrachtungen ist die Anzahl der Parameter von zwei (der Cubic-1Funktion) bis auf 14 (Quintic-4-Funktion) gestiegen. Die Geschwindigkeit der Konvergenz des GA war nur unbedeutend geringer. Dieses ist die besondere Stärke der EM und der wesentliche Grund für die Wahl als Optimierungsalgorithmus.

\subsection{Die Paretofronten mehrsektionaler Profile konstanter Dicke}

Im letzten Abschnitt ist ausschließlich der Zusammenhang zwischen Geometriedefinition und Qualität der Paretofront behandelt worden. Deswegen ist es jetzt interessant, den Zusammenhang der Geometrie zu der aerodynamischen Güte der Profile anhand der Paretofront festzustellen. Denn die Paretofront wird aus Profilen gebildet, die für die jeweilige Geometriedefinition einen optimalen Punkt in dem $\mathrm{C}_{1}-\mathrm{C}_{\mathrm{d}}$-Diagramm darstellen. Das bedeutet, daß geometrische Abhängigkeiten bestimmter Parameter der Profile gegeben sind. Es ist zu vermuten, daß für die gesamte Paretofront sich die Parameter der Profile auf bestimmte Art und Weise einstellen. Es wird im folgenden noch interessant sein, diesen Sachverhalt anhand verschiedener Paretofronten zu untersuchen.

Für eine Untersuchung dieser Art ist es sinnvoll, eine feste prozentuale Profildicke vorzugeben, denn damit lassen sich gewünschte Profileigenschaften festhalten. Im Zuge eines Optimierungsverfahrens würden sich ohne Einschränkungen in der Geometrie die Parameter so einstellen, daß die Profile für industriellen Anwendungen inattraktiv wären. Im unteren Bereich der Paretofront z.B. entstehen extrem schlanke Profile, die den geringsten Widerstand und nahezu keinen Auftrieb, hervorrufen. Diese Profile sind sicher in dem oben untersuchten Rahmen theoretisch interessant, für den Entwurfsingenieur haben sie aber zu wenig Bezug zur Praxis. Um die hier gefundenen Ergebnisse wirtschaftlich nutzen zu können, muß man sich an Eckdaten der industriellen Anforderungen orientieren, ohne dabei die erforderliche Freiheit der Parameter für einen GA einzuschränken.

Es ist ohne weiteres möglich, die Profile in einem praxisrelevanten Rahmen zu halten. Hierfür ist es natürlich notwendig, für die Parameter der Funktionen bestimmte Grenzen zu setzen oder überhaupt als Konstante vorzugeben. Diese Konstanten würden dann nicht mehr durch den GA angepaßt. Das schränkt den Rahmen der geometrischen Möglichkeiten für die Profile ein. Aber auch hier ist es möglich, eine Paretofront unter diesen veränderten Randbedingungen zu berechnen. Für die Praxis sind die geometrischen Einschränkungen meist anhand der strukturellen Anforderungen zu bestimmen. Für die nun folgenden Rechnungen setzte ich als geometrische Zwangsbedingung eine Profildicke von 17\%. Alle anderen Parameter sind wie gehabt variabel. Innerhalb dieser einen Zwangsbedingung werden die Paretofronten für verschiedene Funktionen bestimmt.

Die hier eingeführte Zwangsbedingung wird durch feste Werte von $r$ in der $\varphi$,r-Ebene bei $\varphi=\pi / 2$ und $\varphi=3 / 2 \pi$ realisiert. Die Werte sind so gewählt, daß sie in der abgebildeten Ebene, genau auf der Hälfte des Profils eine Dicke von 17\% vorgeben. Die Wölbung der Profile ist damit ebenfalls eingefroren. Die zugehörigen Paretofronten sind in der folgenden Grafik dargestellt. 


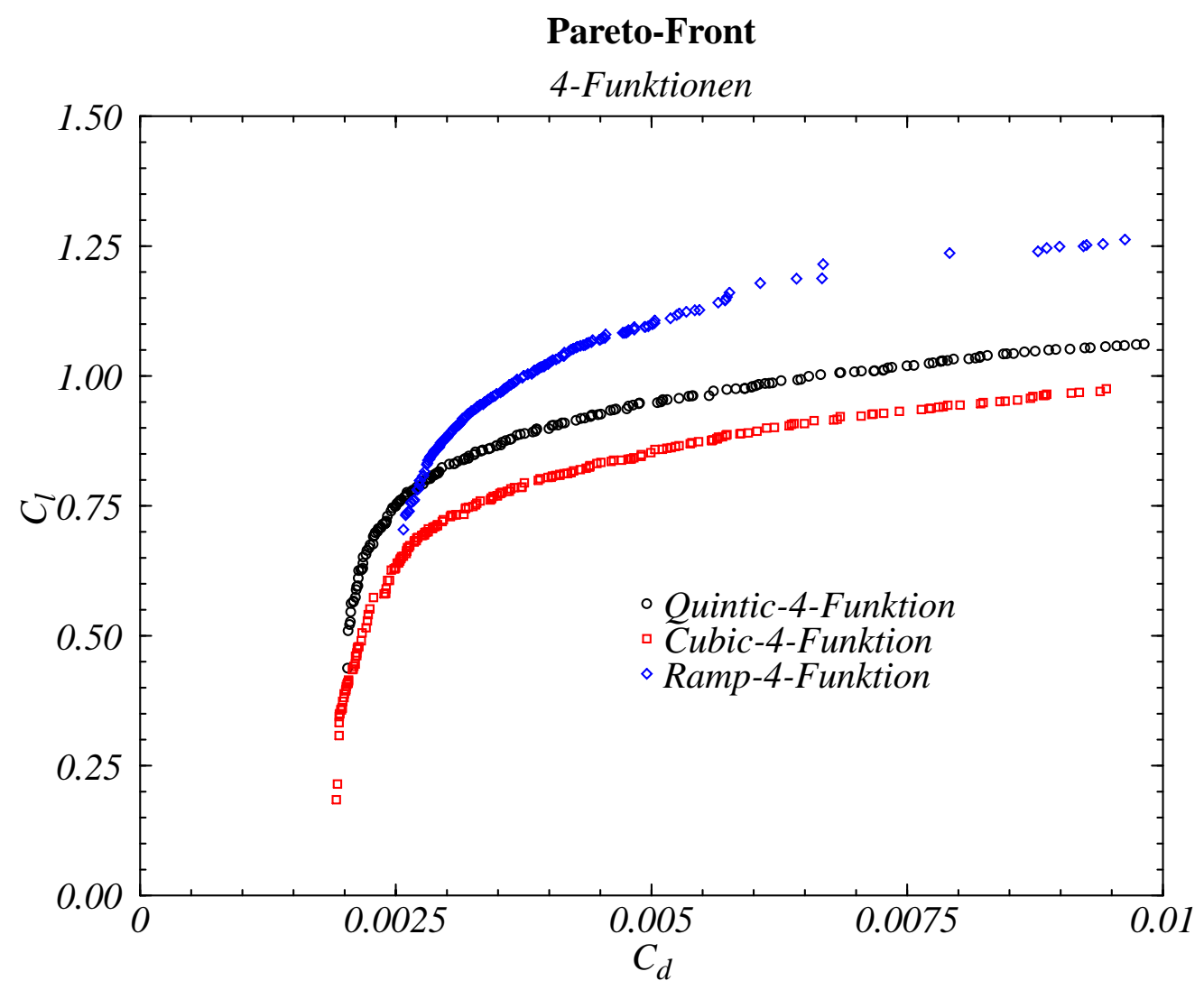

Abb. 19 Paretofronten von drei verschiedenen 4-Funktionen. $M_{\infty}=0.707, \alpha=1.46^{\circ}$.

Die Paretofronten zeigen einen veränderten Verlauf gegenüber den Paretofronten ohne die besondere Einschränkung der 17 prozentigen Profildicke. Hinzugekommen ist hier eine weitere Funktion, genannt Ramp-Funktion. Die Anzahl abhängiger Parameter dieser Funktion entspricht die der Quintic-Funktion. Die Funktion selbst ist anders definiert, siehe Anhang. Die Funktion ist hier eingeführt, damit man eine Alternative zur Quintic-Funktion hat. Beide Funktionen haben die gleiche Anzahl abhängiger Parameter. Hier wird die unterschiedliche Wirkung und der Einfluß der Funktionsdefinition selbst, unabhängig von der Anzahl der Parameter deutlich.

Für die oben geführte Diskussion, die sich auf die Überlegenheit durch erweiterte geometrische Möglichkeiten bezieht, ist Abb. 19 ein interessantes Ergebnis. Der Vergleich der Cubic-4-Funktion und der Quintic-4-Funktion bestätigt über den gesamten Bereich die Ergebnisse, die in einer kurzen Zusammenfassung oben formuliert wurden. Der Verlauf der Paretofronten dieser Funktionen ist nahezu parallel. Aufgrund der überlegenen geometrischen Definitonsmöglichkeiten der Quintic-4-Funktion gegenüber der Cubic-4-Funktion liegt auch deren Paretofront in einem günstigeren Bereich (höher). Ein weiterer Vergleich mit der Ramp-4-Funktion zeigt, daß für sehr hohe Auftriebsbeiwerte noch bessere Ergebnisse als mit der Quintic-4-Funktion zu erzielen sind. Die Definition der Funktion selbst spielt also ebenfalls eine große Rolle. 
Für den Entwurf eines Profils in diesem Bereich der Strömungsparameter (Machzahl, Anstellwinkel) ist das aerodynamische Optimum von Bedeutung. In Abb. 20 ist sichtbar, daß das aerodynamische Optimum für alle drei Funktionen unterschiedlich ist.

$$
C_{l} / C_{d}=f\left(C_{d}\right)
$$

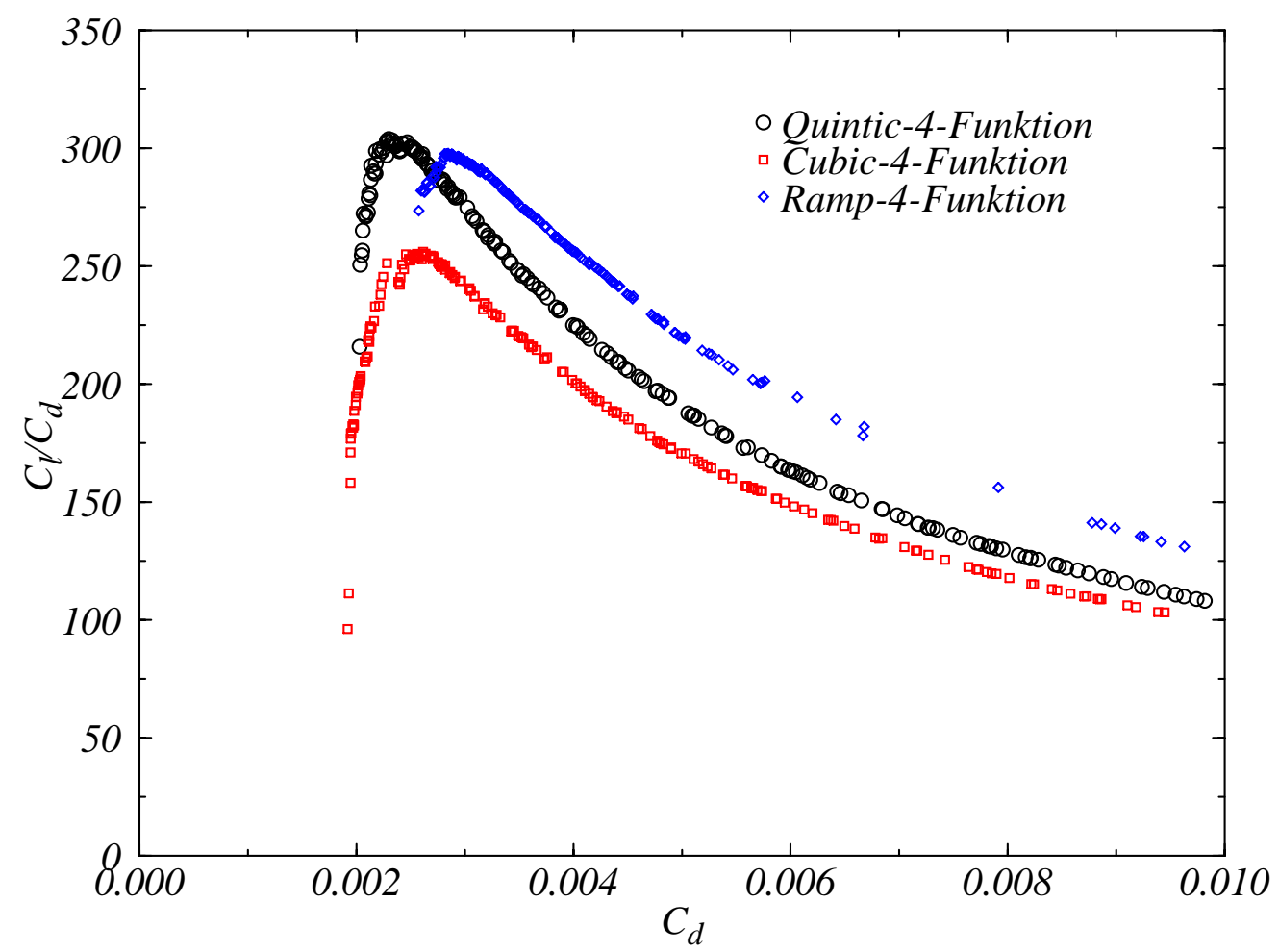

Abb. 20 Aerodynamisches Optimum aus den 4-Funktionen. $M_{\infty}=0.707, \alpha=1.46^{\circ}$.

Für einen günstigen aerodynamischen Profilentwurf unter den gegebenen Strömungsparametern wäre hier ein Profil aus der Quintic-4-Funktion zu wählen, welches im aerodynamischen Optimum liegt. Das Optimum dieser Funktion liegt besser als die der beiden anderen Funktionen.

Nach den hier vorgestellten Ergebnissen erscheint der Versuch, ein vorhandenes Profil in seiner aerodynamischen Leistungsfähigkeit verbessern zu wollen, vor allem mit Hilfe der evolutionären Methoden vielversprechend. Üblicherweise liegen Profile in einer Punktmenge vor. Oft ist das Bestreben der Wissenschaftler ein so gegebenes Profil an aktuellere Anforderungen anzupassen. Mit geringfügigen geometrischen Manipulationen, auch unter veränderten aerodynamischen Verhältnissen, verspricht ein GA gute Ergebnisse zu liefern. Die hier gemachten Erfahrungen mit der Paretofront sprechen dafür. Denn geringfügige geometrische Manipulationen bedeuten, daß der genetische Vektor, der quasi der Repräsentant der geometrischen Möglichkeiten ist, vergrößert wird. Durch diese erweiterten Parameter entstehen erweiterte geometrische Möglichkeiten. Diese erweiterten geometrischen Möglichkeiten werden durch einen GA nahezu vollständig ausgelotet. Das Fazit ist damit wie folgt. Wird der genetische Vektor 
verändert (die geometrischen Möglichkeiten erweitert), ist es ratsam, zuerst die Paretofront des neuen genetischen Vektors zu bestimmen. Diese gibt die maximal zu erreichenden aerodynamischen Möglichkeiten wieder. Eine intuitive Profiländerung „von Hand“ kann bei tiefgehenden aerodynamischen Kenntnissen dem GA den Weg weisen und das Entwurfsverfahren deutlich abkürzen.

\subsection{Qualität mehrsektionaler Profile konstanter Dicke}

Nachdem der Verlauf der Paretofronten hinreichend diskutiert ist, kann nun eine Interpretation der Geometrie und der Druckverteilungen auf den Profilen erfolgen. Wie oben schon angedeutet, kann jetzt der Zusammenhang der Geometrie zu der aerodynamischen Güte der Profile anhand der Paretofront festgestellt werden. In einem ersten Schritt gebe ich hierzu eine Übersicht in Abb. 21. Die hier aufgetragene Paretofront entspricht der Quintic-4-Funktion in Abb. 19.

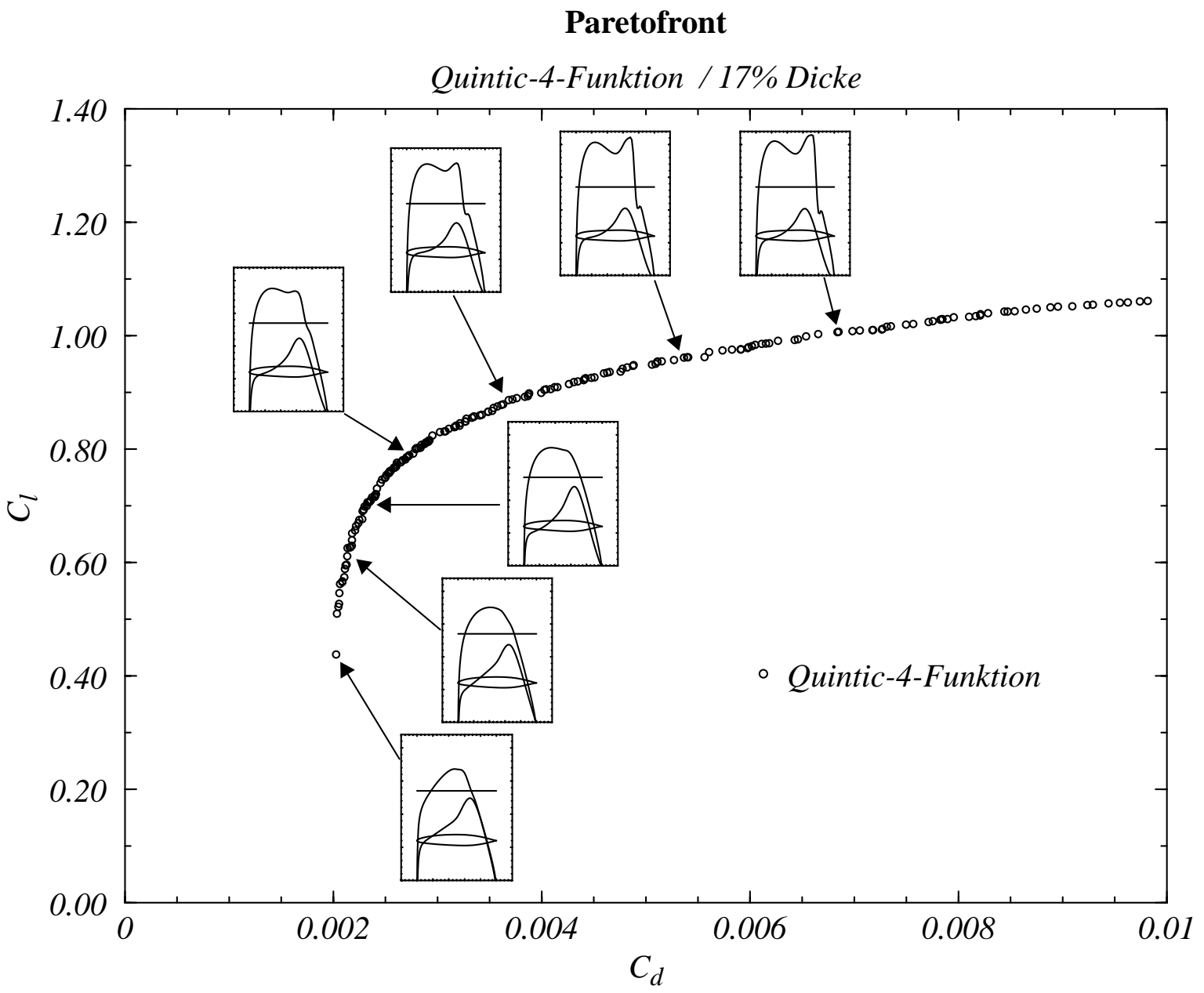

\footnotetext{
Abb. 21 Paretofront der Quintic-4-Funktion mit Ausschnitten von Profilen und der $\mathrm{C}_{\mathrm{p}}$-Verteilung für verschiedene Bereiche.
} 
In Abb. 21 ist zunächst qualitativ die Geometrie der Profile mit der $\mathrm{C}_{\mathrm{p}}$-Verteilung über die Paretofront sichtbar. Durch die Auftragung des kritischen $\mathrm{C}_{\mathrm{p}}$-Wertes innerhalb der ikonisierten Abbildungen ist zu erkennen, daß alle Profile bei den angegebenen Strömungsparametern ein lokales Überschallgebiet besitzen. Besonderes Augenmerk kann hier auf den Verlauf der $\mathrm{C}_{\mathrm{p}^{-}}$ Linie an jeweils der Profiloberseite gelegt werden. Die Profile unterhalb von $C_{l} \approx 0.75$ zeigen einen nahezu stoßfreien Abschluß des Überschallgebietes. An dieser Stelle sei erwähnt, daß ich das Wort „nahezu“ verwende, weil ein GA prinzipiell keine perfekten Lösungen liefert, aber stets beliebig gute Lösungen produzieren kann.

Welche Bedeutung hat die hier gemachte Beobachtung bezüglich der stoßfreien Profile? Die Anwendung eines GA auf die in dieser Arbeit beschriebene Geometriedefinition ließ von Anfang an auf optimal gestaltete Profile hoffen. Daß diese Methode die Profile aber so effizient gestalten würde, ist einigermaßen überraschend. Die Profile im unteren Bereich der Paretofront sind dabei weniger kritisch in ihrer Gestaltung. Denn die Überschallgebiete sind hier noch recht klein, und die Rekompression läßt sich ohne Verluste und stoßfrei realisieren. Schwieriger ist die Gestaltung der Profile zu höheren Auftriebsbeiwerten hin. In dem Bereich nahe dem aerodynamischen Optimum sind stoßfreie Profile nur durch ganz spezielle Verfahren zu verwirklichen, siehe dazu Sobieczky [41].

Stoßfreie Profile treten in der Paretofront nicht als besonderes Phänomen auf. Vielmehr treten stoßfreie Profile bei den hier simulierten reibungsfreien Strömungen bis hin zum aerodynamischen Optimum als mehr oder weniger selbstverständlich auf. Die geometrischen Möglichkeiten sind hier durch den GA offensichtlich ausgeschöpft. Der Verdichtungsstoß als Abschluß des lokalen Überschallgebietes auf der Profiloberseite ist der Grund für den Widerstandsanstieg. Denn dieser wird von dem Algorithmus vermieden, bis die Geometrie dazu nicht mehr in der Lage ist. Ab einem Wert von $C_{l} \approx 0.75$ scheint der Verdichtungsstoß unvermeidbar. Dieser bildet sich im weiteren Verlauf der Paretofront immer weiter aus. Dabei steigt der Widerstand schnell an, und die Paretofront strebt für $\mathrm{C}_{\mathrm{d}} \rightarrow \infty$ gegen nur mehr schwach wachsende Werte von $\mathrm{C}_{1}$.

Hier offenbart sich die enttäuschende Tatsache, daß bei dem Euler-FLOWer bei verschwindendem Stoß noch ein Restwiderstand existiert $\left(C_{d} \sim 0.002\right)$. Bei reibungsfreier Profilströmung müßte in diesem Fall der Widerstand aber identisch Null sein. Ganz offensichtlich ist dieser Fehler numerischer Natur und in der Implementation des 2D-Euler-FLOWer begründet. Zu diesem Zeitpunkt kann ich das Manko des FLOWer nur zur Kenntnis nehmen und auf diesen Fehler hinweisen (siehe Abb. 29). Die Anwendung der evolutionären Methoden wird dadurch nicht in Frage gestellt.

Bei der vorliegenden Geometriedefinition sind die Form und die Lage der Paretofront plausibel. Der Algorithmus formt die Profile so, daß negative Effekte wie der Verdichtungsstoß solange vermieden werden, wie es irgend möglich ist. In diesem Zusammenhang ist es interessant, daß der GA auf einen existierenden Stoß reagiert und die Geometrie derart umgestaltet, daß dieser vermieden wird. Dabei folgt die Berechnungsmethode ausschließlich der algorithmischen Vorschrift. Spezielles aerodynamisches Wissen ist vordergründig nicht implementiert. Dennoch reagiert die evolutionäre Methode auf einen ungünstigen aerodynamischen Effekt.

Ab einem gewissen Punkt kann der GA die Profile nicht mehr stoßfrei anpassen, weil eine Grenze geometrischer Gestaltungsmöglichkeiten erreicht ist. Ab diesem Punkt ist der Stoß nicht 
mehr zu vermeiden, wenn die geometrische Definition beibehalten wird und der genetische Vektor nicht erweitert wird. Ab hier kann dann durch geometrische Variationen der Auftrieb nur noch geringfügig erhöht werden, der Widerstand steigt dann überproportional an. Die Paretofront bildet damit auf ihrer ganzen Länge die Grenze der geometrischen Gestaltungsmöglichkeiten der Profile.

\subsection{Paretofront und Profilpolaren der Entwurfsbeispiele}

Aus der oben besprochenen Theorie zur Paretofront geht hervor, daß die Paretofront eine Einhüllende bildet. Sie bildet im $\mathrm{C}_{\mathrm{l}}-\mathrm{C}_{\mathrm{d}}$-Diagramm eine Grenze maximaler aerodynamischer Leistungsfähigkeit bei Nutzung aller zur Verfügung stehenden geometrischen Mittel. Jede willkürlich gewählte und beliebig kleine, geometrische Variation des Profils muß einen Wert von $\mathrm{C}_{1}, \mathrm{C}_{\mathrm{d}}$ hinter der Paretofront ergeben. Ebenso wird jede Variation des Profilanstellwinkels zu ungünstigeren Werten führen, das zeigt die Profilpolare.

Den Zusammenhang der Paretofront mit der Profilpolaren zeigt die Abb. 22. Die Profilpolare ergibt sich aus der Variation des Anstellwinkels. Diese Variation kann man als geometrische Abweichung vom zugehörigen Profil der Paretofront interpretieren. Wie in der Abbildung sichtbar ist durch diese Art der Variation keine Verbesserung in der Leistungsfähigkeit eines Profils zu erreichen. In der Abbildung sind zwei verschiedene Profile zur Berechnung der Profilpolaren ausgewählt worden (rote und grüne Linie). Die Variation des Anstellwinkels führt in beiden Fällen umgehend zu Werten von $\mathrm{C}_{1}, \mathrm{C}_{\mathrm{d}}$, die hinter der Paretofront liegen. Die Differenz im Anstellwinkel zu den Punkten, die dem Wert auf der Paretofront am nächsten liegen, betragen $1.46^{\circ}+0.02^{\circ}$ und $1.46^{\circ}-0.05^{\circ}$.

Der Ausgangspunkt $\left(\mathrm{C}_{1} / \mathrm{C}_{\mathrm{d}}=\max \right)$ der beiden Profilpolaren liegt stets auf der Paretofront. Dieser Sachverhalt kann für alle Profile der Paretofront angenommen werden. So läßt sich die Paretofront auch als Einhüllende aller Profilpolaren interpretieren.

Die Abb. 22 zeigt allerdings auch, daß die Empfindlichkeit dieser transsonischen Profile gegenüber Änderungen der aerodynamischen Randbedingungen zum Teil sehr hoch ist. Wenn eine geringfügige Änderung des Anstellwinkels, wie oben angegeben, zu deutlichen aerodynamischen Leistungsverlusten führt, steht der praktische Wert des Profils in Frage. Die für die Reisefluggeschwindigkeit von Verkehrsflugzeugen notwendige Toleranz gegenüber der Änderungen aerodynamischer Randbedingungen ist so zunächst nicht gegeben, besonders bei hohem $\mathrm{C}_{1}$. Aber dieses ist ein prinzipielles Problem von transsonischen Konfigurationen. Der transsonische Bereich ist extrem empfindlich gegenüber Änderungen aerodynamischer Randbedingungen. Das gilt insbesondere für eine stoßfreies Überschallgebiet bei relativ großen Auftriebsbeiwerten (hier $C_{l} \approx 0.75$ ). 


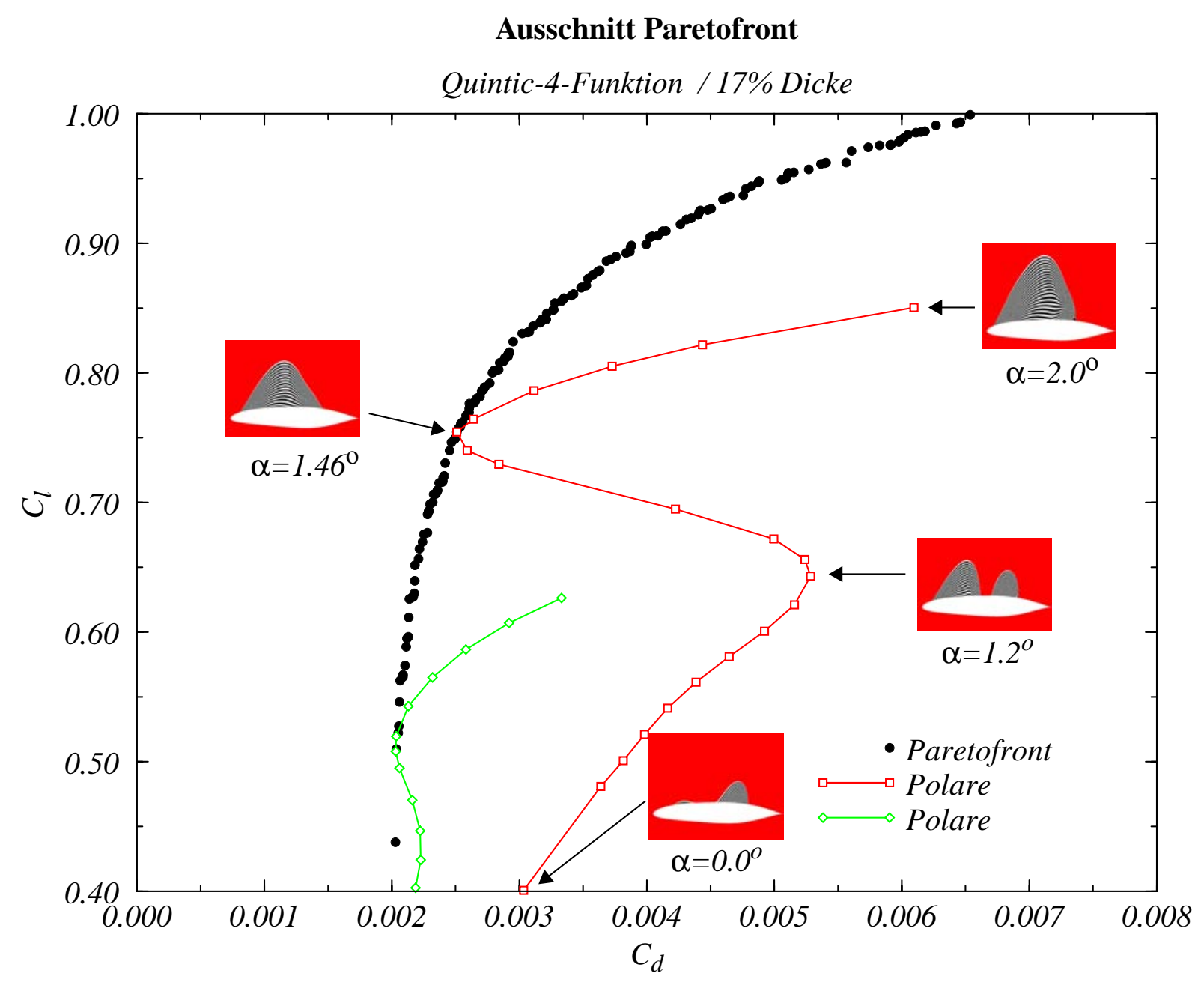

Abb. 22 Paretofront und Profilpolaren. $M_{\infty}=0.707, \alpha=1.46^{\circ}$.

Die Aufgabenstellung für den GA war, die Paretofront zu bestimmen. Daß unter diesen aerodynamischen Randbedingungen (Machzahl, Anstellwinkel) es praktisch wenig Toleranz gegenüber kleinen aerodynamischen Veränderungen gibt, kann der GA bei der Berechnung der Paretofront zunächst nicht berücksichtigen. Die Aufgabe des GA ist es, für verschiedene Auftriebs- und Widerstandsbeiwerte das jeweils optimale Profil zu finden. Diese Aufgabe ist nahezu perfekt erfüllt. Dieser Sachverhalt zeigt, welche robuste Methode der GA ist indem er ohne Rücksicht auf das Phänomen ,Stoßfreiheit“" optimale Profile findet, entweder unter Nutzung der (bekannten) Vorteile stoßfreier Strömung, oder aber den Stoß in Kauf nehmend, wenn offensichtlich keine stoßfreie Lösung mehr möglich ist. Die aerodynamische Wissensbasis hat in den vergangenen Jahrzehnten systematische Methoden entwickelt [41], um auf der Basis theoretisch stoßfreier Strömungen praktisch brauchbare Profile und Tragflügel zu entwickeln. Die hier vorgestellt Methode unter Nutzung des GA enthält die Wissensbasis somit implizit.

Die roten Ikonen in Abb. 22 zeigen die lokalen Überschallgebiete auf den Profilen. Aufgetragen sind dort Linien konstanter Machzahl für $M_{\infty}>1$. Dieses Gebiet enthält ebenfalls die Schallinie. Diese bildet die Grenze zwischen roten Hintergrund und schwarz-weiß gestreiftem Überschall- 
gebiet. Diese Ikone sind für einige Werte des Anstellwinkels dargestellt. Die Paretofront ist für $M_{\infty}=0.707$ und $\alpha=1.46^{\circ}$ berechnet worden. Die Ikone zeigt für $\alpha=1.46^{\circ}$ ein Überschallgebiet mit nahezu stoßfreier Rekompression. Dieses ist das beste Ergebnis, was für dieses bestimmte Profil erreicht werden kann. Eine Erhöhung des Anstellwinkels auf $\alpha=2.0^{\circ}$ zeigt einen sich aufbauenden Verdichtungsstoß. Dieser ist an der zunehmenden Verdichtung der Machlinien im hinteren Bereich des Überschallgebiets zu erkennen. In dem Bereich $1.46^{\circ}<\alpha<2.0^{\circ}$ wird die Strömung auf der Profiloberfläche weiter beschleunigt. Das Überschallgebiet vergrößert sich und schließt mit einem Verdichtungsstoß ab. Dadurch nimmt der Widerstand längs der Profilpolaren in dieser Richtung stetig zu. Der Auftrieb nimmt durch den vergrößerten Anstellwinkel geringfügig zu.

Verringert man den Anstellwinkel $\left(\alpha<1.46^{\circ}\right)$, so fällt das Überschallgebiet mehr und mehr in sich zusammen. Das führt soweit, daß zwei voneinander getrennte, kleinere Überschallgebiete entstehen. Beide Überschallgebiete weisen einen Verdichtungsstoß bei dem Übergang auf Unterschallgeschwindigkeit auf. Dieser Strömungszustand stellt einen bekannten ungünstigen $\mathrm{Zu}$ stand bezüglich der Verringerung des Anstellwinkels dar (drag creep). Hier tritt größerer Widerstand auf. Bei weiterer Verringerung des Anstellwinkels baut sich das vordere Überschallgebiet stetig ab, bis es ganz verschwindet. Zurück bleibt das Überschallgebiet im hinteren Bereich des Profils $\left(\alpha=0.0^{\circ}\right)$. Durch Abbau des vorderen Überschallgebietes fällt auch der ungünstige Effekt des Verdichtungsstoßes in der Profilmitte weg. Damit erfolgt eine Abnahme des Widerstandes. Der Auftrieb nimmt dabei auch ab.

In Abb. 22 ist eine zweite Profilpolare (grüne Linie) eingetragen. Diese zeigt qualitativ denselben Verlauf, wie die Polare des eben besprochenen Profils, aber dieses Profil ist wegen des geringeren Wertes für $C_{1}$ unter optimaler Gleitzahl $\left(C_{1} / C_{d}\right)$ wesentlich gutmütiger. Auch hier tritt keine Überschreitung der Paretofront auf. Zunehmender oder abnehmender Anstellwinkel erreicht nicht die aerodynamische Leistungsfähigkeit des Profils der Paretofront. Es ist anzunehmen, daß für alle Profile der Paretofront dieses Verhalten vorliegt. Der Verlauf der Profilpolaren bestätigt die bisher gefundenen Ergebnisse, daß die Paretofront eine Grenze maximaler aerodynamischer Leistungsfähigkeit bildet.

\subsection{Die Parameter der Sektionen auf der Paretofront}

Das Erreichen der Paretofront ist, ganz allgemein gesprochen, das Erreichen einer Grenze. Da die Parameter der Funktionen und Sektionen dieser Paretofront zugrunde liegen, müßten auch diese Parameter an eine Grenze gestoßen sein. Zumindest kann man annehmen, daß die Parameter einer gewissen Ordnung unterworfen sind und ihre Anordnung in irgendeiner Weise kennzeichnend sein müssen. Das Erreichen der Paretofront sollte sich in den Parametern widerspiegeln. Aufgrund gegenseitiger Wechselwirkung der Parameter unter sich, ist keine strenge Ordnung der Parameter zu vermuten.

Es stellt sich die Frage, ob einige aerodynamische Gesetzmäßigkeiten aus den Parametern abzulesen sind. Denn die Parameter haben über die gewählte Funktion und die konforme Abbildung eine geometrische Bedeutung für die Profile. Diese üben Einfluß auf die aerodynamische Leistungsfähigkeit aus. Die Grenze maximaler aerodynamischer Leistungsfähigkeit ist mit der Paretofront erreicht. Fraglich ist, ob auch die Parameter an eine Grenze gestoßen sind und ggf. 
bekannte geometrische Abhängigkeiten der aerodynamischen Konfiguration wiedergeben. Möglicherweise lassen sich Rückschlüsse auf Abhängigkeiten dieser Parameter und deren Einfluß auf die optimierte Geometrie ziehen.

Um diesen Sachverhalt zu klären, ist in folgenden Abbildungen, pro Abbildung exakt ein Parameter (von der Paretofront der Quintic-4-Funktion mit 17\% Profildicke), in seinem Verlauf über die gesamte Paretofront dargestellt. Die Parameter sind in der $\varphi$,r-Ebene definiert (siehe Abb. 6). In dieser Ebene müssen sie später auch interpretiert werden. Um einen Überblick eines bestimmen Parameters im Verlauf der Paretofront zu gewinnen, sind die Parameter über dem Widerstandsbeiwert dargestellt.

Die Quintic-4-Funktion ist in vier Sektionen unterteilt. Hier werden die Sektionen im mathematisch positiven Sinn gezählt. In der $\varphi$,r-Ebene ist der Bereich $0<\varphi<\pi / 2$, der Bereich des Profils von der Hinterkante zur Mitte der Profiloberseite. Der Punkt $(\pi, r)$ liegt im Nasenpunkt des Profils. Die Sektionen 0-3 teilen den Kreis in Abschnitte zu je $90^{\circ}$ ein. Dieser Kreis wird konform abgebildet. Sinngemäß wird also das Profil in vier Teilabschnitte von oberer Hinterkante über Oberseite und Staupunkt zur unteren Hinterkante eingeteilt. Jeder dieser Abschnitte ist in der $\varphi$, r-Ebene durch die Quintic-4-Funktion und ihrer vier Parameter vorgegeben.

Aufgrund der stetigen Anschlußbedingungen aufeinanderfolgenden Sektionen sind jeweils (im Falle der Quintic-4-Funktion) die beiden letzten Funktionsparameter der vorhergehenden Sektion mit denen der beiden ersten Parametern der darauffolgenden Sektion identisch. Beispielsweise ist also $\mathrm{s}_{2}$ der Sektion 0 identisch mit $\mathrm{s}_{1}$ der Sektion 1.

In Abb. 23 bis Abb. 25 sind die Parameter der Quintic-4-Funktion von 17\%-Dicke über die Paretofront dargestellt. Die jeweils linke Seite zeigt die Tangenten an den oben genannten Nahtstellen, wie sie in Abb. 6 gegeben sind. Die rechte Seite zeigt den Parameter $e$ der Funktion. Dieser bildet die zweite Ableitung (siehe Quintic-Funktion im Anhang) an denselben Nahtstellen.

Insgesamt ist für alle Parameter jeder Sektion die oben vermutete Ordnung gegeben. Alle Parameter zeigen einen charakteristischen Verlauf über die Paretofront. Kein Parameter unterliegt einer totalen ungeordneten statistischen Streuung. Wäre das der Fall, würde das auf einen epistatischen Effekt innerhalb des GA hinweisen. Kurz gesagt hätte der Parameter keinen oder nur geringen Einfluß auf das Ergebnis, daß man auf ihn auch hätte verzichten können. Man hätte durch Verzicht auf diesen Parameter den Parameterraum verkleinern können. Dieser Fall ist hier nicht gegeben.

Ein geringe Streuung bedeutet hier starke Abhängigkeit der Geometrie. Eine größere Streuung weist daraufhin, daß der Parameter in der aktuellen Anströmung keinen hohen Einfluß ausübt. Insgesamt ist es hier nur sinnvoll, relative Werte zu betrachten. Absolute Werte sind hier wegen der konformen Abbildung nur schwer zu diskutieren.

Die Steigung der Tangenten an der Hinterkante ist in Abb. 23 links sichtbar. Für Profile geringer Auftriebsbeiwerte ist sie deutlich flacher, als für Profile hoher Auftriebsbeiwerte. Das steht im Einklang mit Ergebnissen aus der Literatur. Im weiteren Verlauf scheint sich dieser Parameter einem Grenzwert zu nähern. Das bedeutet, daß durch eine stärkere Neigung der Hinterkante, kein weiterer Auftrieb mehr gewonnen werden kann. Der Einfluß der Hinterkante ist damit ausgeschöpft. 

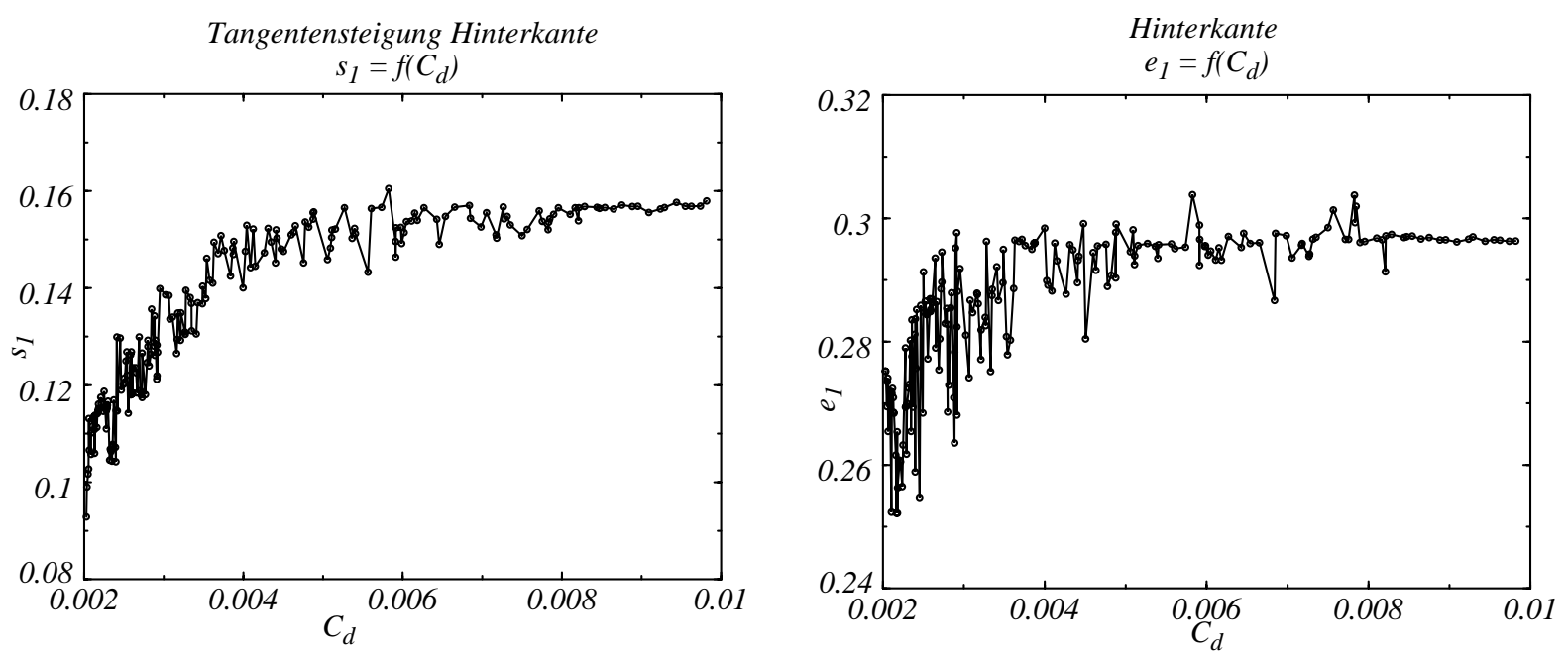

Abb. 23 Parameter der Hinterkante.
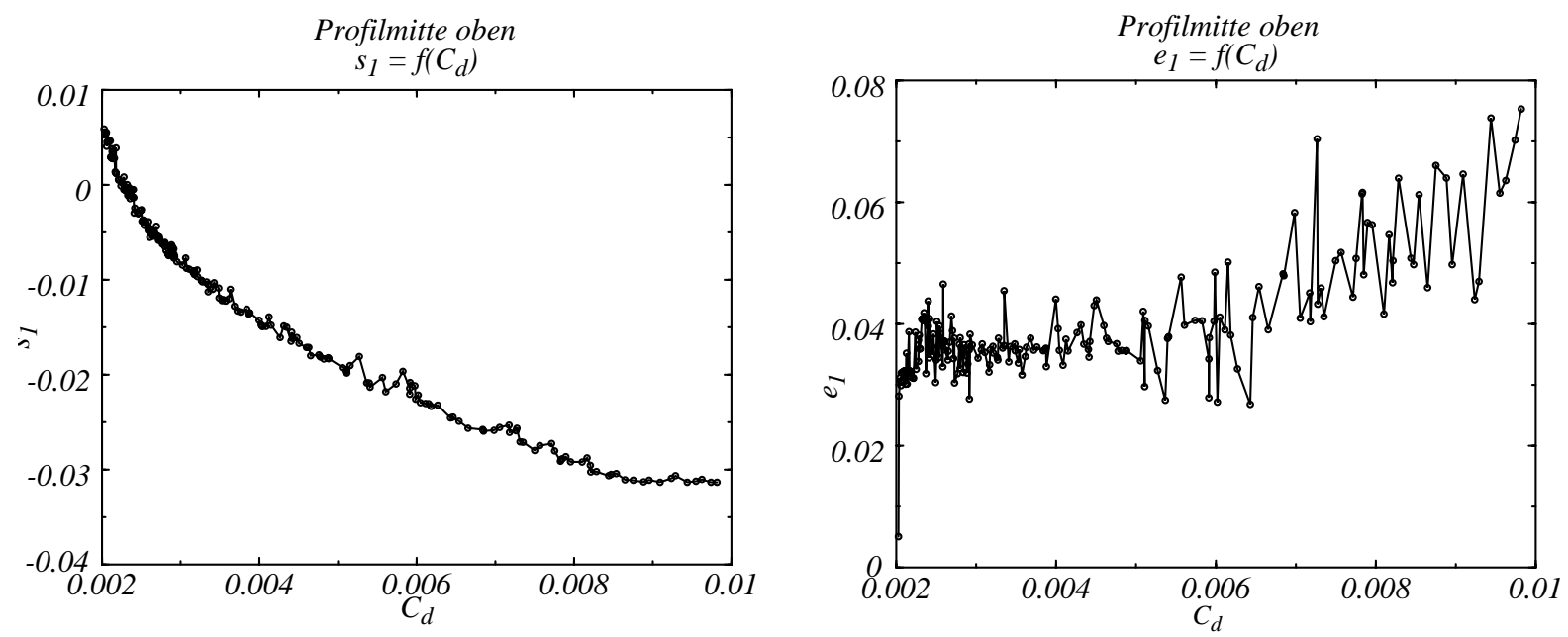

Abb. 24 Parameter in der oberen Profilmitte, im Dickenmaximum.
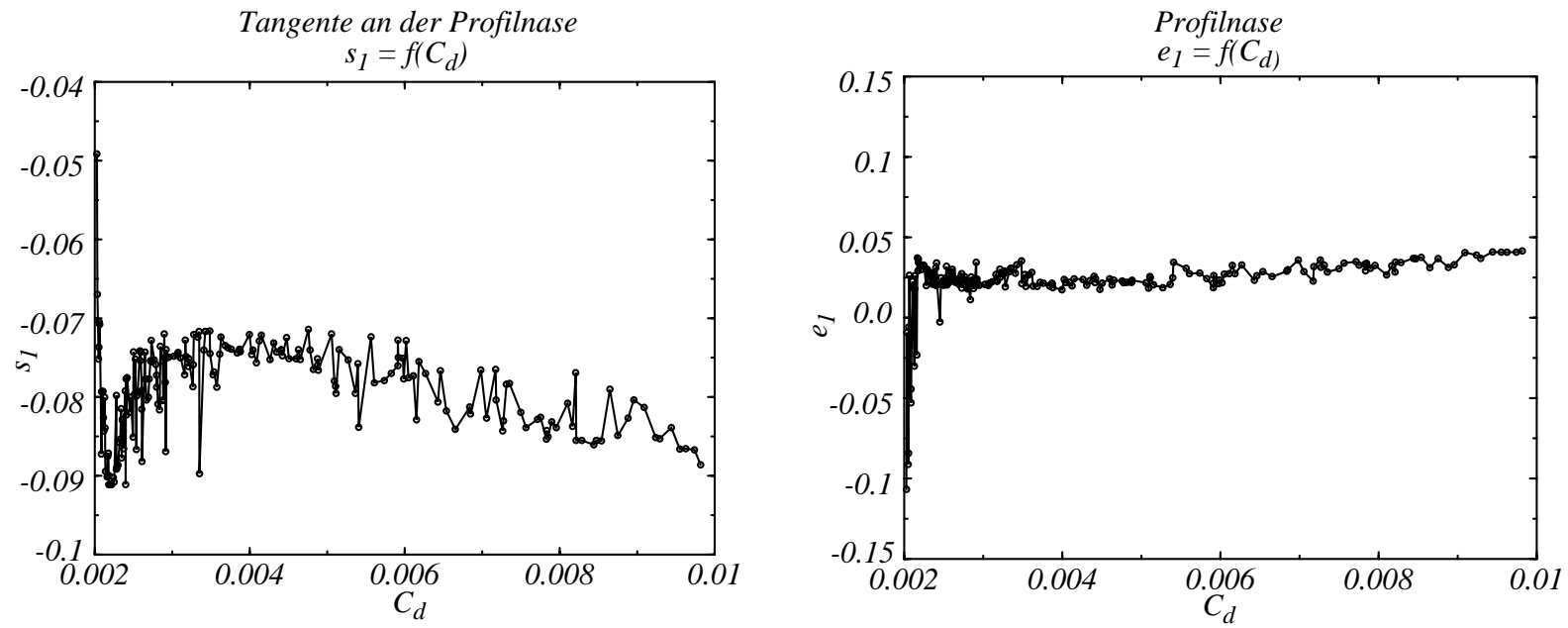

Abb. 25 Parameter an der Profilnase. 
Einen ähnlichen Verlauf hat die Kurve für den Nasenradius in der $\varphi$,r-Ebene in Abb. 26. Für Profile geringen Widerstandes sind die Profilnasenradien klein. Die Strömung auf der Profiloberfläche wird durch kleine Profilnasen sehr viel weniger stark beschleunigt, als durch große Profilnasen. Die Auftriebsbeiwerte sind in diesem Abschnitt der Paretofront klein. Um aber hohe Auftriebsbeiwerte zu liefern, nimmt der Nasenradius schnell zu. Die Strömung wird dadurch auf der Profiloberseite stärker beschleunigt und erzeugt dadurch vermehrt Auftrieb. Auch hier ist eine Art Sättigung für den Parameter im oberen Verlauf der Paretofront zu erkennen. Denn der Nasenradius bleibt über einen weiten Bereich hohen Widerstandes nahezu konstant.

Eine strenge Abhängigkeit des Strömungszustandes von der Geometrie ist in Abb. 24 links zu erkennen. Dieser Parameter stellt die Tangente an dem Dickenmaximum auf der Profiloberseite dar. Offensichtlich reagiert die Strömung auf diesen Parameter sehr sensibel. Ich vermute, daß sich dieser Sachverhalt mit der geometrischen Nähe des Verdichtungsstoßes erklären läßt. Der Verdichtungsstoß trägt wesentlich zum Anstieg des Widerstandes bei. Auf dieses Verhalten reagiert die Geometrie mit hoher Sensibilität.

Im Vergleich dazu betrachte man Abb. 27 links. Diese Abbildung zeigt die Tangente auf der unteren Profilseite im Dickenmaximum. Die Tangente streut hier über die Paretofront sehr viel stärker als die entsprechende Tangente im Dickenmaximum auf der Profiloberseite. Dieser Sachverhalt unterstützt die Annahme, daß die geringe Streuung auf der Profiloberseite mit dem Verdichtungsstoß zusammenhängt.

Die Abb. 28 zeigt die Veränderung der Tangentensteigung an der unteren Profilhinterkante. Die geringe Streuung unterstreicht die sensible Abhängigkeit der Strömung von diesem Parameter. Im Bereich geringer Wiederstandsbeiwerte ist der Wert der Tangente in der $\varphi$,r-Ebene negativ. Das bedeutet geringes, oder gar kein, sog. Rear-Loading. Dieser Wert steigt zunächst schnell, im weiteren Verlauf linear an. Strömungstechnisch bedeutet das, daß die Strömungsgeschwindigkeit im Bereich der unteren Hinterkante verringert wird. Parallel dazu zeigt die Tangente an der oberen Hinterkante einen relativ hohen Anstieg. Die Tatsache deute ich als einen Versuch der Geometrie, die Strömungsgeschwindigkeit auf der Profiloberfläche auf einem hohen Niveau zu halten. Zusammen genommen ergibt die hohe Strömungsgeschwindigkeit auf der hinteren Profiloberfläche relativ zur verlangsamten Strömung an der unteren Hinterkante ein hohes Rear-Loading, d.h. vermehrt Auftrieb im hinteren Bereich des Profils.

Dieses ist eine bekannte Vorgehensweise zur Auftriebssteigerung. Wenn praktisch die Profilgeometrie im vorderen Bereich angepaßt ist, läßt sich durch geschicktes Manipulieren der Tangenten an der Hinterkante der Auftrieb weiter vergrößern. Genau dieser Effekt läßt sich aus dem Verlauf dieser Parameter über der Paretofront herauslesen.

Insgesamt ist zu beobachten, daß die ersten Ableitungen (jeweils links in den Abbildungen) sehr viel weniger streuen als die zweiten Ableitungen (jeweils rechts in den Abbildungen). Eine Ausnahme hiervon ist die Abb. 25. Während der Verlauf der Tangentesteigung der Profilnase über die Paretofront fast eine parabelförmige Gestalt hat, liegen die Werte der zweiten Ableitung alle auf einer Geraden. Diesen Effekt vermag ich an dieser Stelle nicht zu deuten.

Die Parameter der anderen oben verwendeten Paretofronten (siehe Abb. 19 und Abb. 20) sind hier nicht dargestellt. In den sensiblen strömungstechnischen Bereichen zeigen die Parameter ein ganz ähnliches Streuungsverhalten wie die hier diskutierten Parameter der Quintic-4-Funktion. 


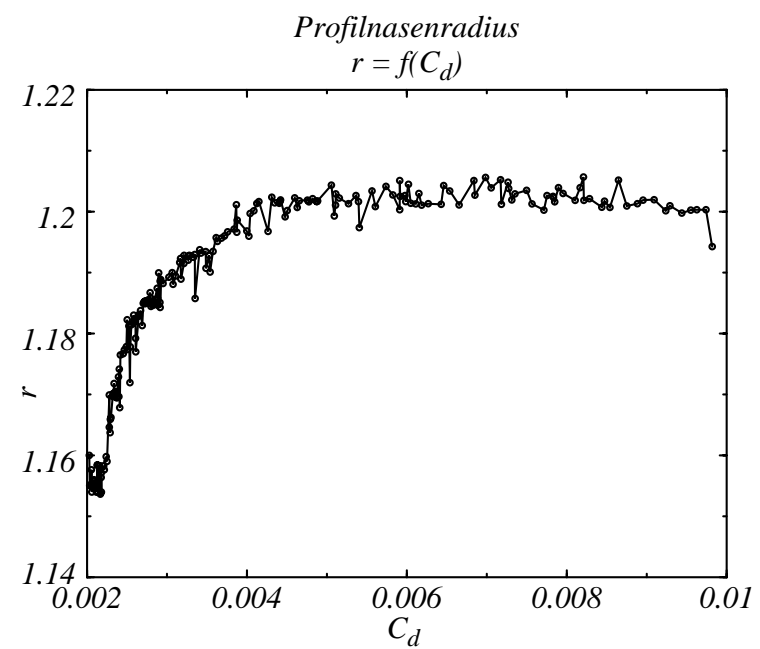

Abb. 26 Nasenradius der Profile.
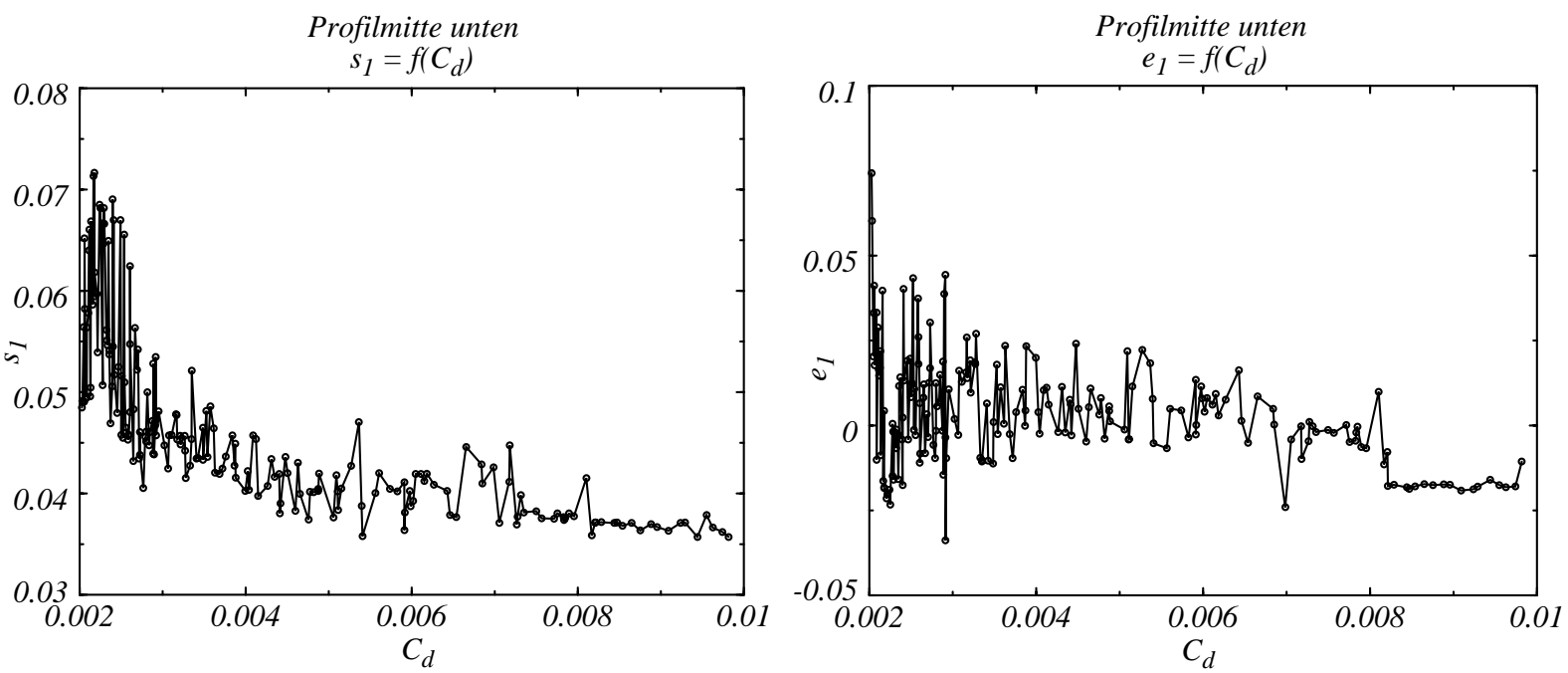

Abb. 27 Parameter in der unteren Profilmitte, im Dickenmaximum.
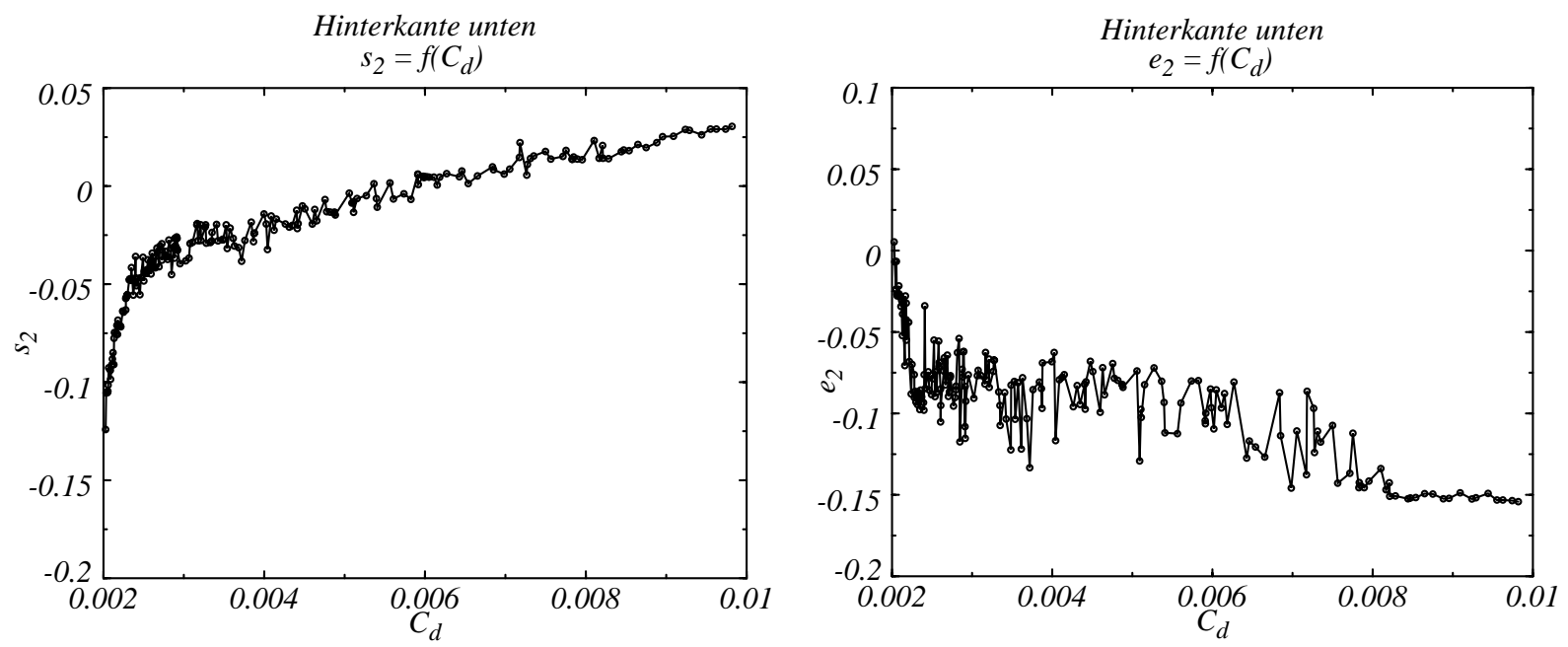

Abb. 28 Parameter der Hinterkante. 


\subsection{Paretofront aus Navier-Stokes-Verfahren}

Für reale Profilauslegungen sind Strömungen mit Berücksichtigung von Reibungseffekten interessant. Die Reibung beeinflußt den Charakter der Strömung in der Grenzschicht wesentlich. Außerhalb der Grenzschicht kann bei hohen Reynoldszahlen die Strömung als nahezu reibungsfrei angenommen werden. Die Strömung in der Grenzschicht selbst ist unter diesen Anströmungsbedingungen voll turbulent. Siehe dazu auch Abb. 9.

Im einem ersten Schritt zur Auslegung der Profilgeometrie genügt es, wenn man mit Hilfe der Eulergleichungen gute Druckverteilungen am Profil erzielt. Lassen diese Druckverteilungen auf eine stoßfreie Rekompression des Überschallgebietes schließen, so wird in der Regel auch bei einer reibungsbehafteten Strömung die Grenzschicht keine Ablösungserscheinungen am Profil zeigen. Der Verdichtungsstoß ist eine der Hauptursachen für das Ablösen der Strömung am geometrischen Ort des Verdichtungsstoßes oder verfrüht im Bereich der Hinterkante.

Bei Berücksichtigung von Reibungseffekten liegen die aerodynamischen Beiwerte schlechter als bei reibungsfreier Betrachtung. Deswegen liegt die gesamte Paretofront für reibungsbehaftete Rechnungen vollständig unterhalb der Paretofront für reibungsfreie Rechnungen.

\section{Paretofront}

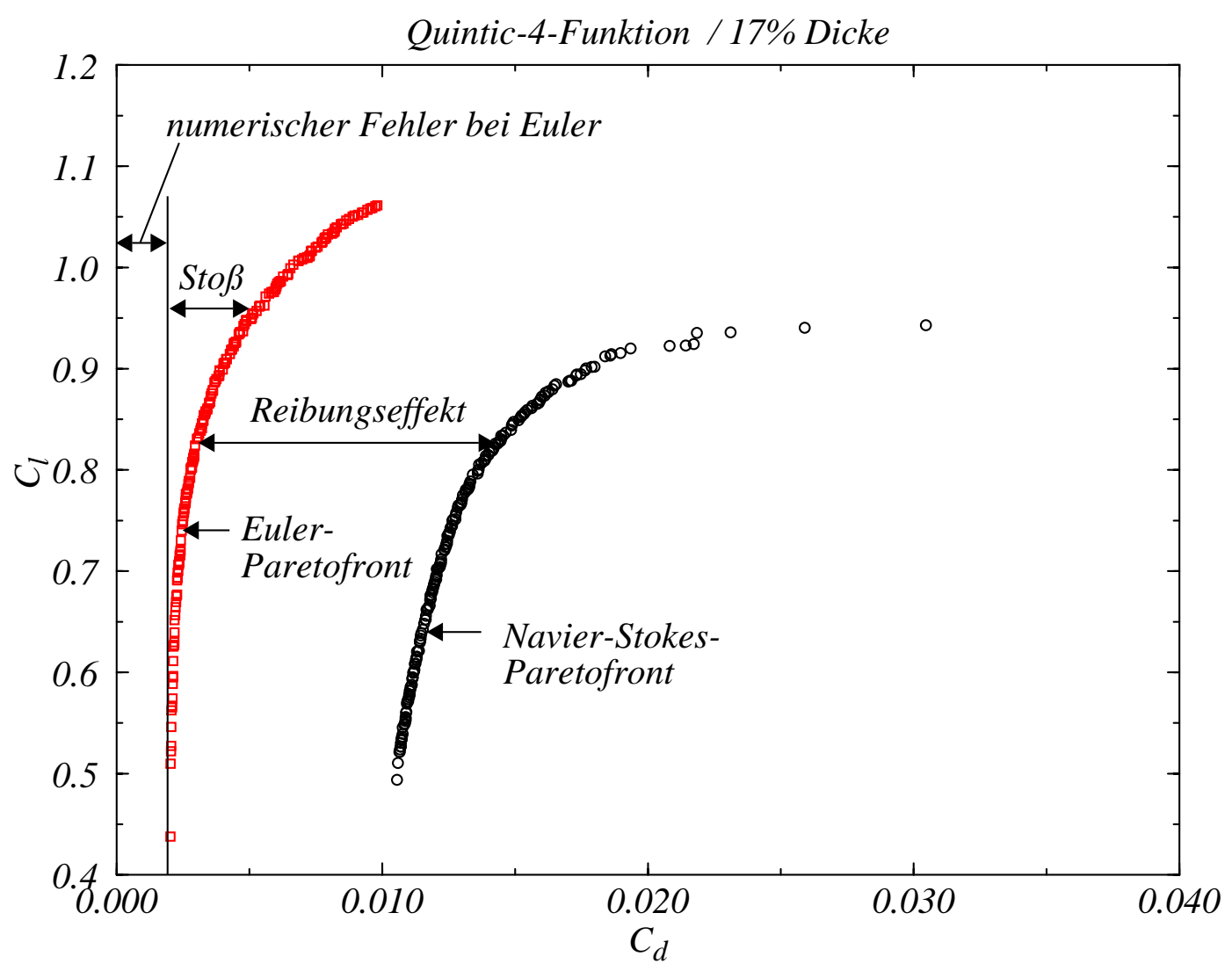

Abb. 29 Paretofronten der Quintic-4-Funktion für Strömung mit und ohne Reibung. $M_{\infty}=0.707, \alpha=1.46^{\circ}, R_{e}=10^{6}$. 
Die Abb. 29 zeigt die Paretofront bei Berücksichtigung von Reibungseffekten. Diese Abbildung entspricht der Abb. 21 für eine reibungsfrei angenommene Strömung. Der Verlauf der Fronten ist in der kurzen Zusammenfassung am Ende von Kapitel 6.3 schon erwähnt worden. Denn die Form der Paretofront ist abhängig von dem verwendeten Verfahren zur Strömungsfeldberechnung. Wenn unterschiedliche Verfahren unterschiedliche physikalische Phänomene berücksichtigen, hat natürlich auch die Paretofront einen anderen Verlauf.

Die Betrachtung der Profilgeometrie entlang der Paretofront in Abb. 29 zeigt grundsätzlich dasselbe Verhalten, welches im Abschnitt 6.5 schon diskutiert wurde. Bis zum aerodynamischen Optimum zeigen die Profile keinen Verdichtungsstoß. Ab dem aerodynamischen Optimum ist der Verdichtungsstoß durch die Geometrie nicht mehr zu vermeiden. Der Stoß bildet sich stetig weiter aus und erhöht dadurch den Widerstand am Profil. Die Stoßentwicklung ist parallel zu den reibungsfrei angenommenen Profilen in Abb. 21. Der Stoß ist hier allerdings weniger scharf. Ich führe das auf einen gewissen Dämpfungseffekt der Grenzschicht zurück, (StoßGrenzschicht-Inteferenz).

Die Abb. 30 zeigt ein Profil aus dem aerodynamischen Optimum der Navier-Stokes-Rechnungen. Dieses zeigt einen ebenso sauberen Druckverlauf an Profilober- und -unterseite, wie dies schon in früheren Eulerrechnungen der Fall war.

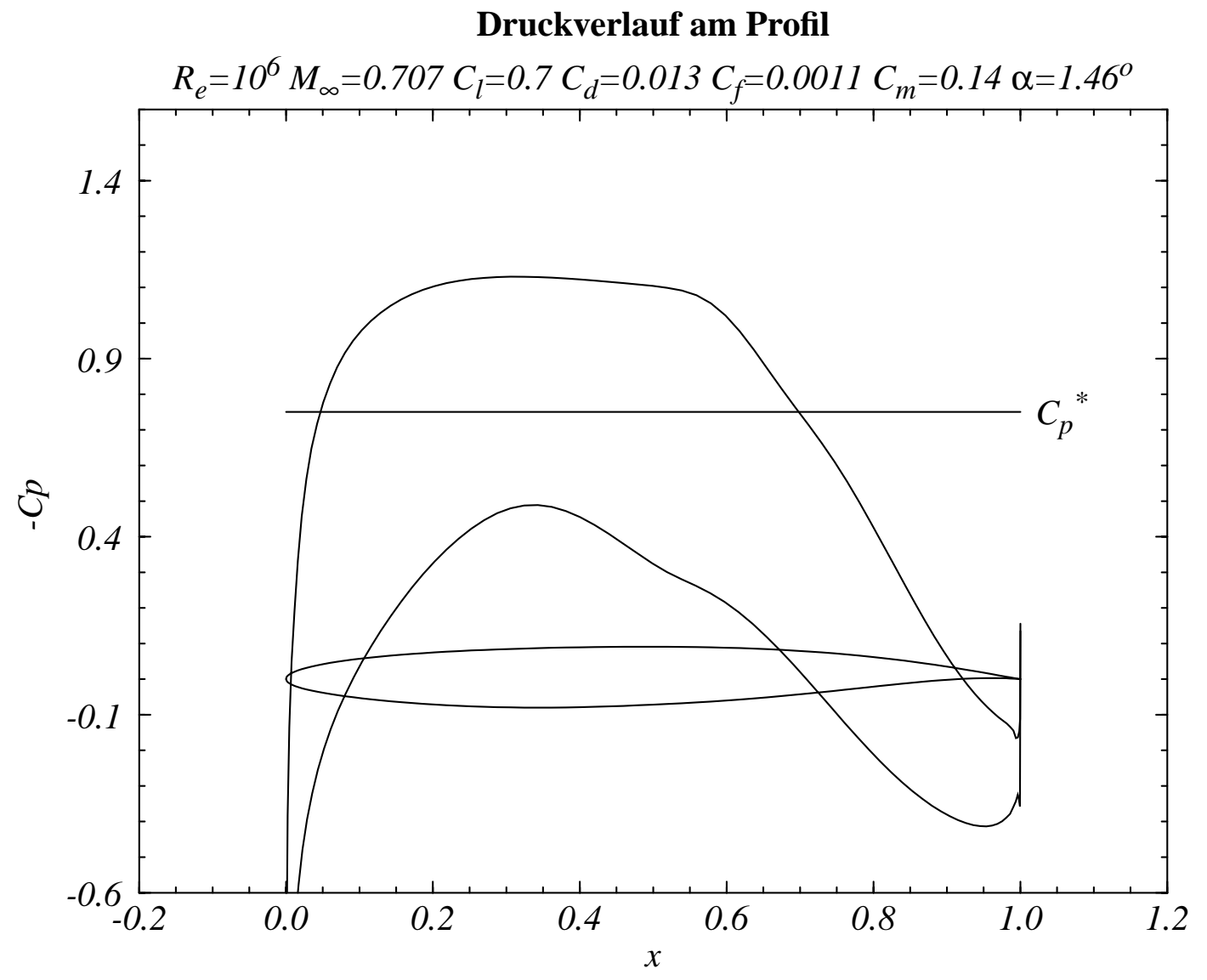

Abb. 30 Stoßfreies Profil aus reibungsbehafteter Rechnung. $M_{\infty}=0.707, \alpha=1.46^{\circ}, R_{e}=10^{6}$. 
Die bisher vorgestellten Ergebnisse bestätigen die Vermutung, daß die genetische Optimierung unabhängig vom jeweiligen Strömungslöser ist. In den Eulerrechnung wurden ebenso gute Resultate erzielt wie in den hier angewendeten Navier-Stokes-Rechnungen. Allerdings ist eine Navier-Stokes-Rechnung wesentlich zeitaufwendiger.

Die $\mathrm{C}_{\mathrm{p}}$-Verteilung der Profile der Paretofronten zeigen im aerodynamischen Optimum eine nahezu stoßfreie Rekompression der Überschallgebiete. Die Entwicklung einer solchen Kontur ist für reale reibungsbehaftete Strömungen sonst nur mit erheblichem theoretischen Aufwand und großem Fachwissen zu erzielen, siehe dazu [39]. Auf der Paretofront entstehen sie quasi als natürliche Geometriekonfiguration zwischen Profilen mit kleinem lokalen Überschallgebiet, und Profilen hohen Auftriebs mit scharfem Stoß.

Der Versuch, ein mit den hier vorgestellten Geometriewerkzeugen entworfenes Profil in seinen aerodynamischen Eigenschaften zu verbessern, indem man das Profil durch dieselben geometrischen Methoden definiert und dann mit Hilfe des GA optimiert, muß scheitern oder wird das entsprechenden Profil auf der Paretofront reproduzieren. Die Paretofront ist das zu erreichende Optimum. Alle Profile, wenn sie denn dieselbe Geometriedefinition haben, konvergieren gegen diese Front. Deswegen kann ein entworfenes Profil nur mittels einer verfeinerten Geometriedefinition weiter verbessert werden.

\subsection{Anwendung der Evolutionären Strategie auf eine existierende Paretofront}

Neben dem GA existiert eine weitere evolutionäre Optimierungsmethode. Diese ist die Evolutionäre Strategie (ES). Tatsächlich gestaltet sich die Anwendung des ES als sehr viel schwieriger als die Anwendung des GA. Deswegen spielt sie in dieser Arbeit auch nur eine untergeordnete Rolle. Für die Art und Weise der hier vorgenommenen Profiloptimierung ist der GA die bessere Vorgehensweise. Insbesondere ist die Konvergenzgeschwindigkeit des GA für diese Problemstellung erheblich höher als die der ES. Es muß betont werden, daß die Fragestellung, ob ein GA oder eine ES bessere Ergebnisse liefert, erst durch das Ergebnis selbst beantwortet werden kann. Es ist a priori nicht zu entscheiden, welche Methode zu bevorzugen ist.

Aber für die Mehrfachoptimierung bezüglich der Paretofront ergeben sich bei der ES sofort Schwierigkeiten bei der adaptiven Schrittweitenregelung (7). Denn wie soll man hier entscheiden, ob insgesamt eine aerodynamische Verbesserung aller Profile eingetreten ist? Die Schrittweitenregelung sagt grob aus, daß der Streuungsparameter vergrößert werden muß, wenn eine Verbesserung von mehr als 20\% der Profile eingetreten ist. Diese Entscheidung ist hier schwierig , da man eine Paretofront als Ergebnis erhält. Da man über einen großen Bereich von $\mathrm{C}_{1}, \mathrm{C}_{\mathrm{d}}$ eine Bewertung vornimmt, und die Paretofront aus möglichst gleichmäßig verteilten Punkten bestehen soll, stellt sich die adaptive Schrittweitenregelung als problematisch heraus. Denn wie soll man eine Verbesserung dann überhaupt definieren?

Diese Definition der aerodynamischen Verbesserung der Profile fällt leichter, wenn bereits eine durch eine GA berechnete Paretofront existiert. Als Population für die Anwendung einer ES kann dann eine kleinere Zahl von Profilen von der Paretofront entnommen und verändert werden. Die Verbesserung definiert man dann darüber, wieviele Profile auf welcher Seite der Paretofront liegen. Liegen 20\% der Profile jenseits der Paretofront im guten Bereich, dann kann der Streuungsparameter künstlich vergrößert werden. 
Per definitionem kann kein Objekt die Paretofront überschreiten. Das gilt für eine theoretisch perfekte Paretofront. Da aber die Paretofront hier numerisch bestimmt wird, existiert eine minimale Differenz zwischen der theoretischen und der numerisch berechneten Paretofront. Denn in einem Abschnitt schlechter Besetzung mit Punkten auf der Paretofront können noch geringfügig bessere Ergebnisse erzielt werden. Diese Differenz auf einem Minimum zu senken, ist der Beitrag, den eine ES hier noch leisten kann. Allerdings ist dieser Beitrag gering. Denselben Effekt könnte man einfach durch Erhöhung der Anzahl der Objekte erzielen, die durch den GA berechnet werden. Allerdings steigt dann auch der Rechenaufwand drastisch.

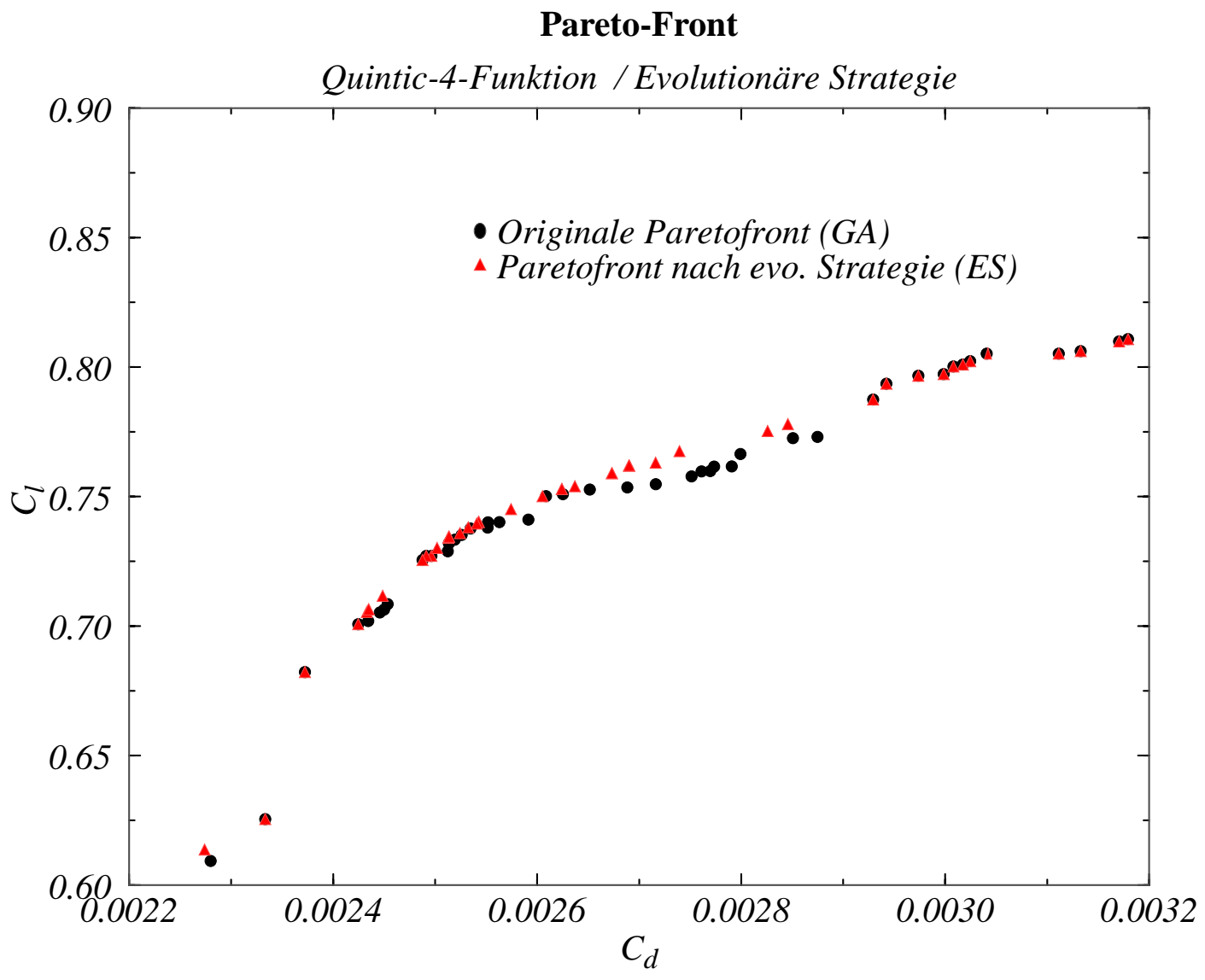

\section{Abb. 31 Verbesserung der Paretofront durch die Evolutionäre Strategie.}

Die schwarzen Punkte bilden die originale Paretofront, die durch den GA berechnet wurde, hier im Ausschnitt zu sehen. Die roten Dreiecke bilden die Paretofront nach Anwendung der ES. Es ist deutlich zu sehen, daß es eine Verbesserung in manchen Bereichen der Paretofront gibt (hier bei $C_{l} \approx 0.75$ ). Ebenso deutlich ist, daß die Verbesserungen nur graduell vorhanden sind und eher Lücken der Paretofront füllen, als die Paretofront selbst in Frage stellen. Die Verbesserungen sind gering. Wie oben schon erwähnt, ist eine perfekt glatte Paretofront nur durch eine sehr hohe Zahl von Profilen zu erreichen. Und selbst dann sind dünne Besetzungen der Paretofront nur durch erweiterte GA-Techniken zu vermeiden. Die ES kann im diesem Umfeld recht gut als letztes Mittel zur Verbesserung bestimmter Punkte genutzt werden, bringt aber keinen evidenten Vorteil oder gar andere Ergebnisse als der Ansatz durch den GA. 
Der GA und die ES stellen ein wirkungsvolles Instrument zur Optimierung für diese Art von Anwendung dar. Insbesondere erscheint der GA die geeignetere Methode, da die Paretofront sehr viel schneller erschlossen wird. Die ES entwickelt ihre Stärke erst im Nachhinein, um eventuell aufgetretene Lücken der Paretofront zu schließen. Der GA erforscht und bewertet schnell ein großes Gebiet hoch dimensionaler Vektorräume, ist aber bei der Berechnung bei hoher Punktdichte gegenüber der ES leicht im Nachteil. Die ES hingegen erschließt mehrdimensionale Räume sehr langsam, ist aber in der Feinabstimmung der Geometriedefinition teilweise überlegen. Allerdings ist der Aufwand zur guten Abstimmung der ES (Streuung) deutlich höher, weshalb der GA die geeignetere Methode zu sein scheint. 


\section{Ergebnisse für Tragflügelkonfigurationen im Unterschall}

Für allgemeine Profiloptimierung existieren unzählige wissenschaftliche Beiträge. Hinsichtlich genetischer Optimierung ist die Zahl relativ begrenzt. Besonders aktiv auf diesem Gebiet zeigt sich die Gruppe um Obayashi. Hier finden sich interessante Entwicklungen auf dem Gebiet der Optimierung aerodynamischer Konfigurationen mit Hilfe der genetischen Algorithmen.

Generell ist jedoch die geometrische Definition an sich vernachlässigt. In vielen Arbeiten besteht die geometrische Definition von Profilen stets aus einer Vielzahl von Punkten, die mit einer evolutionären Methode in ihrer geometrischen Anordnung optimiert werden. Eine Ausnahme ist bei Oyama [31] gegeben, der die Geometriedefinition von Sobieczky in [43] verwendet. Die grundlegenden Funktionen und die Idee zur Geometriedefinition in dieser Art und Weise sind zum Unterschied zur in der vorliegenden Arbeit verwendeten Joukowski-Abbildung auf Formen für Profile und Flügel direkt im physikalischen Raum angewandt. Insbesondere die analytische Konturdefinition für spezielle aerodynamische Anwendungen ist hier tiefgehend entwickelt.

Durch die genetischen Algorithmen ist die Berechnung einer Paretofront möglich. Es läßt sich hiermit eine große Zahl von Parametern oder Parameterlinien variieren und ein Optimum bestimmen. Damit lassen sich beliebige aerodynamischen Konfigurationen in ein weiteres Umfeld einbetten. Denn bisher galten aerodynamische Konfigurationen als akzeptabel, wenn sie quantitativ gute Ergebnisse lieferten. Durch die Existenz einer Paretofront muß diese Regel erweitert werden. Denn die Konfigurationen sind nur dann akzeptabel, wenn sie auf der Paretofront liegen (also die geometrischen Möglichkeiten ausgeschöpft sind). Somit lassen sich auch geringe Abweichungen von der theoretisch optimalen Konfiguration untersuchen und beurteilen.

Die in den vorangegangenen Kapiteln gemachten Erfahrungen und Erkenntnisse sollen hier für die dreidimensionale Tragflügelauslegung genutzt werden. Damit erhöht sich der Schwierigkeitsgrad deutlich. Denn wenn man von einer konstanten Profilierung über die Spannweite absehen will, stellt sich sofort das Problem, die Profilierung über die Spannweite in geeigneter Weise zu definieren. Die geometrische Definition des Tragflügels sollte für den GA ebenso geeignet sein wie für die Profilierung. Zusätzlich zur Profilierung kommt jetzt der Konturverlauf des Flügelgrundrisses hinzu. Für eine Tragflügelauslegung ist auch noch eine Variation des Anstellwinkels über die Spannweite (Verwindung des Flügels) erforderlich.

Die Erweiterung auf drei Dimensionen wirft auch für die Optimierung durch den GA neue Fragen auf. Denn bei der Variation aller eben genannter Parameter darf man dennoch nicht grundsätzliche Prinzipien des GA außer Acht lassen. Eines der wesentlichen Prinzipien besagt, daß alle Parameter grob denselben Einfluß auf das Ergebnis haben müssen. Ob dieser vorgegebene Rahmen bei den geometrischen Anforderungen hier einzuhalten ist, muß noch eruiert werden.

Insgesamt sollte die Geometriedefinition nicht von der bisher benutzten Darstellung abweichen. Denn eine einheitliche analytische Darstellung aller geometrischen Randbedingungen wäre wünschenswert. Bei Sobieczky in [42] findet sich diese einheitliche Darstellung, die sich schon für schwierige aerodynamische Problemstellungen bewährt hat. 


\subsection{Tragflügelgrundriß (Planform)}

Für ein Studium der Tragflügelprofilierung eignet sich in besonderer Weise der Grundriß eines elliptischen Tragflügels endlicher Spannweite. Dieser Tragflügel erzeugt nach der Prandtlschen Integralgleichung [36] den geringsten induzierten Widerstand bei inkompressibler Strömung. Eine elliptische Zirkulationsverteilung läßt sich am unverwundenen Tragflügel durch eine elliptische Tiefenverteilung über die Spannweite erzeugen. Durch die elliptische Zirkulationsverteilung entsteht hinter dem Tragflügel eine konstante Abwärtsgeschwindigkeit des Mediums. Dieser Effekt minimiert die induzierten Wirbel an den Tragflügelenden. Die Leistungsverluste durch diese induzierten Wirbel sind damit ebenfalls minimiert.

Aus dieser theoretischen Betrachtung läßt sich nun die Tragflügelkontur entwerfen. Dazu wähle ich zwei Halbellipsen, die an der 1/4-Linie zusammengefügt sind. Durch diese geometrische Konstruktion wird bei elliptischer Tiefenverteilung und konstantem Anstellwinkel über die Spannweite wiederum eine elliptische Zirkulationsverteilung reproduziert.

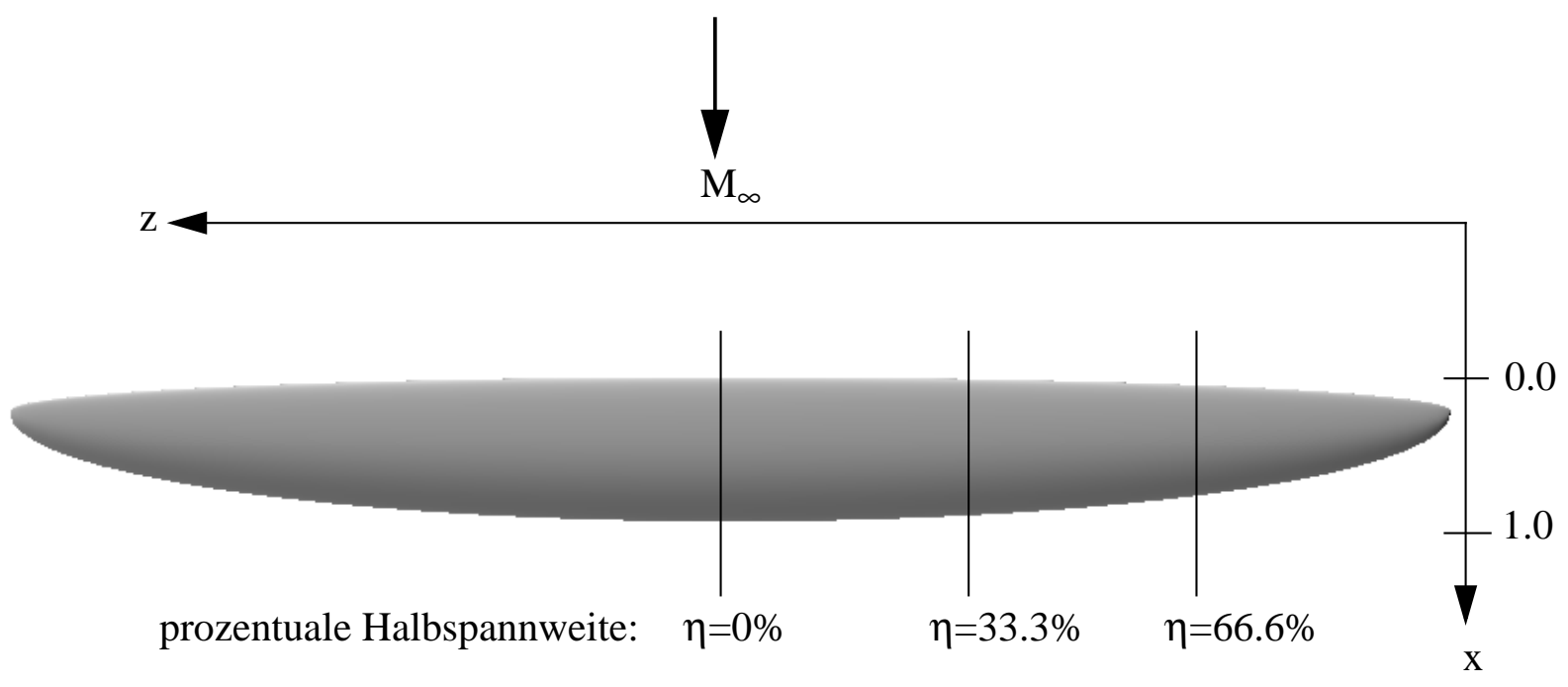

Abb. 32 Elliptischer Tragflügel.

Der Vorder- und Hinterkantenverlauf läßt sich bequem durch die erwähnten Funktionen vorgeben, siehe Abb. 8. Hierzu kann die Ramp-Funktion verwendet werden (siehe Anhang). Damit ist die geometrische Konstruktion der Kontur ebenfalls durch die schon oben verwendeten Funktionen möglich und kann durch den GA, in gleicher Weise wie die Grundfunktionen für die Profilierung, angepaßt und optimiert werden. In Abb. 32 ist so ein elliptischer Tragflügel der Streckung 1:10 dargestellt. Die eingezeichneten Schnitte der prozentualen Halbspannweite werden im folgenden für die Darstellungen der $\mathrm{C}_{\mathrm{p}}$-Verteilung über den Spannweite benötigt. Der Grundriß bleibt zunächst für weitere Auswertungen fest. 


\subsection{Tragflügelprofilierung}

Die Auslegung einer Tragflügelkonfiguration geschieht in der Regel durch Einpassen verschiedener Profile in einen vorgegebenen Tragflügelgrundriß. Hier werden Vorderkante und Hinterkante als Kurven vorgegeben und geeignete Profile in diese Kontur eingepaßt. Bisherige Techniken beschränkten sich darauf, die Anpassung der Profile über die Spannweite durch ein Mischungsverhältnis der Profile zu realisieren. Diese Methode ist in den geometrischen Möglichkeiten auf wenige Grundprofile festgelegt und stößt schnell an Grenzen.

Eine vollständige analytische Methode zur Tragflügelprofilierung ist in Kapitel 4.2 beschrieben. Diese Methode vereinigt eine flexible Gestaltung bei gleichzeitiger guter Kontrolle der Geometrie. Dieses sind wichtige Vorraussetzungen für die Optimierung durch einen GA.

Die Tragflügelprofilierung selbst bildet neben der Vorderkanten -und Hinterkantenkontur sowie den Verlauf des Verwindungswinkels und evtl. eines Dickenparameters einen der wichtigtsten Gestaltungsparameter. Im weiteren Verlauf wird sich noch zeigen, ob überhaupt die Möglichkeit gegeben ist, diese Gestaltungsparameter alle gleichzeitig einer Optimierung zu unterziehen.

Erwähnenswert in diesem Zusammenhang ist, daß der Profilentwurf für den Tragflügel (siehe Abb. 7) vollständig unabhängig von jeder Konturdefinition ist. Die Profile werden erst in einem zweiten Schritt in den vorgegebenen Tragflügelgrundriß eingepaßt. Der Grundriß und die Profilierung werden unabhängig voneinander genetisch manipuliert (optimiert). Die Profilierung bildet im Grunde einen selbständigen Teil der Optimierung. Dieses Vorgehen gilt sowohl für den Profilentwurf aus den ebenen Lösungen als auch für den Entwurf aus der in Kapitel 4.2 vorgestellten Methode.

\subsubsection{Elliptischer Tragflügel mit konstanter Profilierung}

Die klassische Methode zur Profilierung eines Tragflügels ist, ein geeignetes Profil aus den ebenen Lösungen in einen Tragflügel mit sehr großer Streckung einzufügen. Die Behandlung dieses Tragflügels reduziert die dreidimensionalen Probleme auf zwei Dimensionen. Denn ist in der dritten Dimension keine Änderung der Kontur oder der Profilierung gegeben, so ändert sich auch das Strömungsfeld in dieser Richtung nicht. Der Schluß von ebenen Lösungen auf dreidimensionale Konfigurationen ist dennoch nur in engen Grenzen zulässig. Ein Beispiel dafür wäre ein Rechteckflügel großer Streckung, weil bei diesem dann die Randeffekte in ihrer Wirkung stark reduziert sind.

Der oben skizzierte elliptische Tragflügel bildet ein typisches Objekt mit praxisrelevanter Spannweite. Der folgende Abschnitt untersucht kurz die Möglichkeit in die oben gegebene Konturlinie für Vorder- und Hinterkante des elliptischen Tragflügels eine konstante Profilierung einzufügen. Die konstante Profilierung stammt einmal in der Form eines optimiertes Profils aus den bisher berechneten Paretofronten, im zweiten Fall entwerfe ich eine konstante Profilierung als Optimum eines GA. Den Ausgangspunkt der Untersuchungen bildet ein unverwundener elliptischer Tragflügel mit dem Streckungsverhältnis 1:10 und mit konstanter Profilierung im Unterschall. 


\subsubsection{Tragflügelgestaltung durch optimierte Profile}

Zunächst ist festzustellen, in wie weit sich ein Profil aus den bisher berechneten Paretofronten als Grundlage für das Flügelprofil eignet. Das entspricht der klassischen Vorgehensweise. Sollte hier schon ein befriedigendes Ergebnis erzielt werden, dann kann sich die gesamte Optimierung auf Profile beschränken, die dann als Grundlage der Geometriedefinition eines vollständigen Tragflügels ausreicht. Nimmt man theoretische Erkenntnisse der Tragflächentheorie hinzu und gibt der Tragfläche durch die Vorgabe des Grundrisses eine elliptische Auftriebsverteilung, dann sollte mit dieser Methode schon ein gutes Ergebnis vorprogrammiert sein. Für diesen Versuch entleihe ich ein Profil aus vorher berechneten Paretofronten nahe dem aerodynamischen Optimum. Der Leistungsbereich und die strömungstechnischen Daten des genannten Profils in reibungsfreier Strömung sind in Abb. 33 dargestellt.

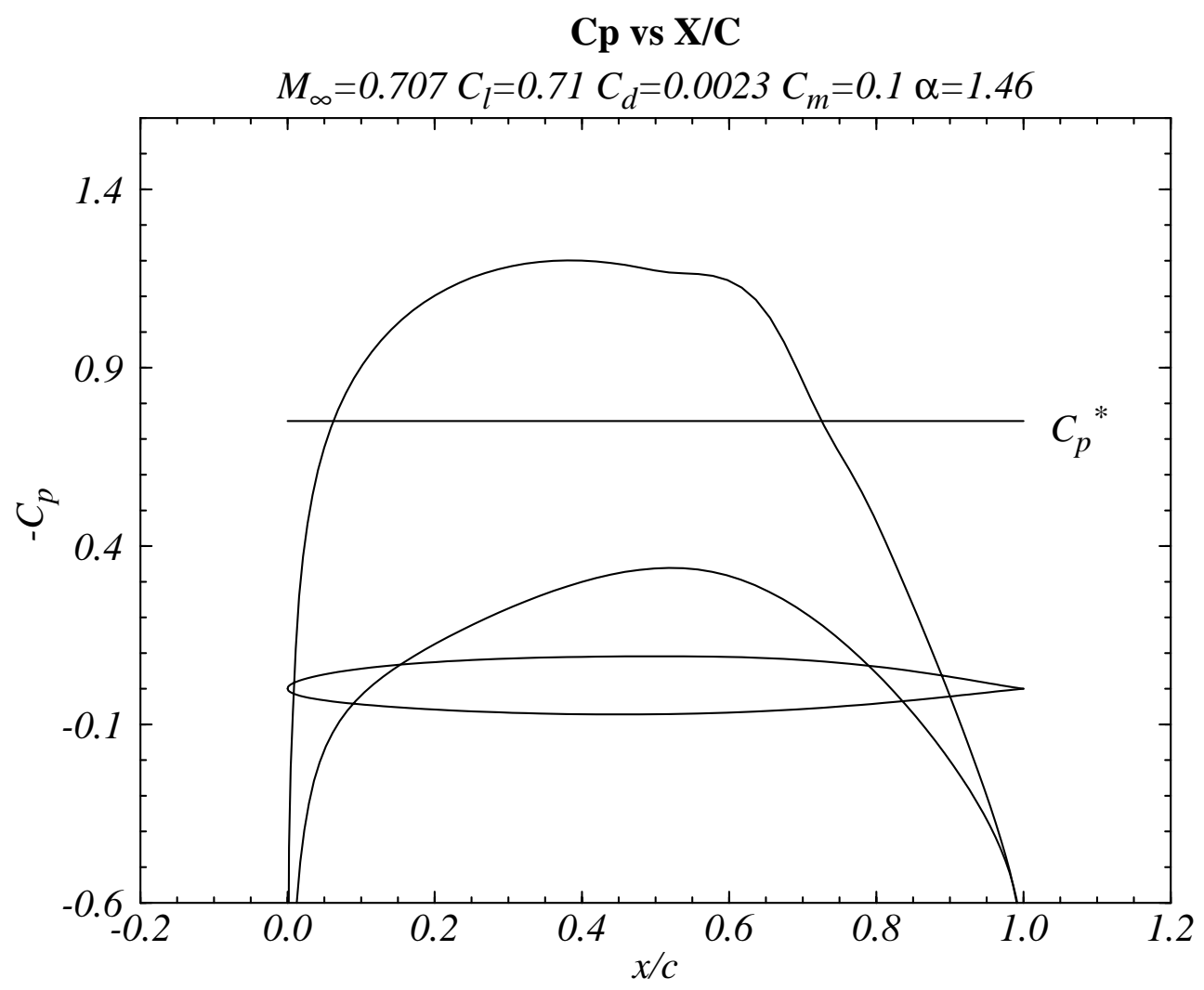

\section{Abb. 33 Optimiertes Profil aus der Paretofront.}

Der Vergleich der Abbildungen Abb. 33 und Abb. 34 zeigt das Ergebnis aus der ebenen und dreidimensionalen Lösung nach Einpassen des Profils in einen Tragflügel. Dieses Profil verwende ich in diesem Beispiel als Grundmuster zur Tragflügelprofilierung.

Das Ergebnis aus der dreidimensionalen Eulerrechnung des Tragflügels fällt wie erwartet aus. Siehe dazu Abb. 34. Hier ist die $\mathrm{C}_{\mathrm{p}}$-Verteilung an den Schnitten der prozentualen Halbspannweite, wie in Abb. 32 angegeben, dargestellt. Der Tragflügel hat eine endliche Streckung. Das bedeutet, daß hier induzierter Widerstand aufgrund abgelöster Randwirbel an den Tragflügelenden auftritt. Dadurch erhöht sich relativ zum ebenen Profil bei gleichem Anstellwinkel der Widerstand. Und dadurch nimmt auch der Auftriebsbeiwert in gleicher Relation ab. Deswegen 
ist es zulässig beim Tragflügel, um den Strömungszustand mit dem ebenen Fall vergleichen zu können, den Anstellwinkel ein wenig zu vergrößern, um den selben Auftriebsbeiwert zu erhalten.

Weiter werden in der Berechnung nach dem Eulerverfahren natürlich kompressible Effekte berücksichtigt, die in der klassischen Theorie nicht enthalten sind.

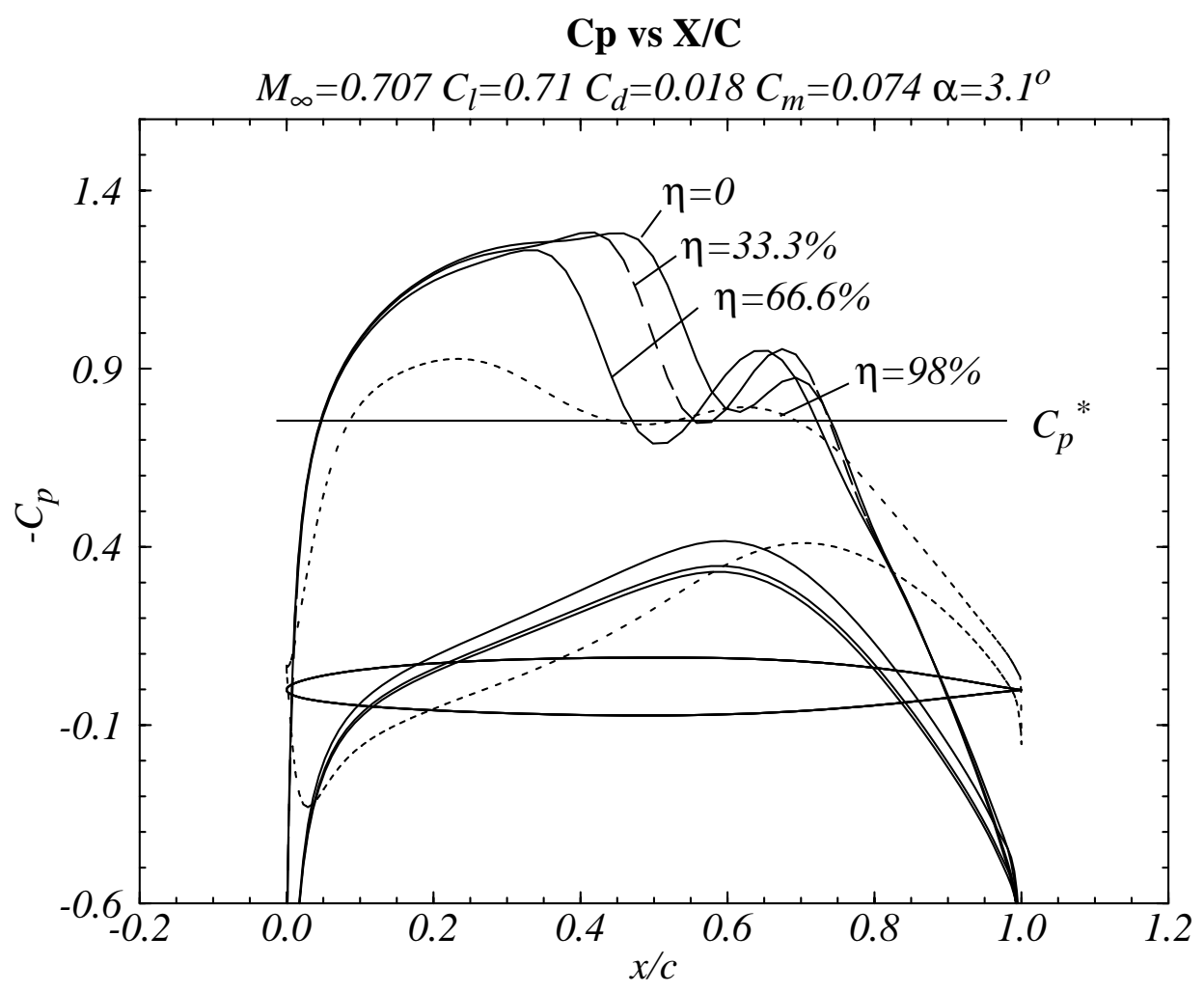

\section{Abb. $34 \mathrm{C}_{\mathrm{p}}$-Verteilung an den verschiedenen spannweitigen Stationen im Querschnitt des Tragflügels.}

Der Vergleich der $\mathrm{C}_{\mathrm{p}}$-Verteilungen, so man diesen Vergleich ziehen darf, zeigt sofort die Grenzen auf, die diese Vorgehensweise hat. Die $\mathrm{C}_{\mathrm{p}}$-Verteilung des optimierten Profils findet sich nicht in der $\mathrm{C}_{\mathrm{p}}$-Verteilung des Tragflügels wieder. Die Druckverteilung am Tragflügel zeigt eine leichte Nachexpansion, der bei weiterer Erhöhung des Anstellwinkels ein Verdichtungsstoß folgt (hier nicht aufgelöst). Dieses Ergebnis deutet daraufhin, daß die Verwendung ebener Problemstellungen als Grundmuster für eine Tragflügelprofilierung im Transsonikbereich nur eine allererste Nährung ergibt: Die klassische Theorie beeinhaltet keine kompressiblen Effekte. Die Unterschiede in der Druckverteilung werden weitgehend auf kompressiblen Effekten beruhen. Durch die Unfähigkeit, eine stoßfreie Profilierung geeignet anzupassen, können negative Effekte nicht ausgeblendet werden. Dies läßt sich mit den zur Verfügung stehenden Mitteln und dem Optimierungsverfahren durch einen GA schnell überprüfen. 


\subsubsection{Tragflügelgestaltung durch Genetischen Algorithmus}

Eine konstante Profilierung ist mit den entwickelten geometrischen Möglichkeiten leicht möglich. Die rote Linie in Abb. 7 zeigt, wie die Tragflügelprofilierung konstant gehalten werden kann. Ist diese Linie über die Spannweite eine Gerade, dann gibt es keine Veränderung dieses Parameters längs der Spannweite. Mit der Höhe der Geraden parallel zur z-Achse verändern sich die Parameter in Spannweitenrichtung gleichmäßig. Auf diese Weise ist eine konstante Profilierung gewährleistet. Die Profilierung kann nur noch in diesem strengen Rahmen angepaßt und optimiert werden.

Läßt man im Gegenteil hierzu eine flexible Profilierung in Spannweitenrichtung zu, kann das durch eine der schon genannten Funktionen (Cubic, Quintic) realisiert werden. Die erwähnte rote Linie Abb. 7, die ich hier im folgenden als Parameterlinie bezeichne, hat dann einen flexiblen Verlauf. Sie besteht (hier) in Spannweitenrichtung nur aus einer Sektion. Die Parameter werden für die Profilierung von dieser Parameterlinie abgelesen. Ist diese Linie über die Spannweite nicht konstant, ist auch die Profilierung über die Spannweite nicht konstant. Da die Parameterlinien aber differenzierbar und stetig sind, ist auch die Profiloberfläche frei von Unebenheiten oder gar Krümmungssprüngen.

Ob hiermit die geometrischen Gestaltungsparameter zu weit eingeschränkt sind, kann nur das Ergebnis zeigen. Insbesondere die konstante Profilierung unterliegt erheblichen Einschränkungen. Die Strömungsgrößen werden wie in allen anderen Berechnungen vorgegeben. Nur der Anstellwinkel wird hier auf $\alpha=3.0^{\circ}$ gesetzt. Denn dieser Anstellwinkel reproduzierte zumindest annährend die $\mathrm{C}_{\mathrm{p}}$-Verteilung aus dem ebenen Profilierungsansatz.

Das Ergebnis zeigt Abb. 35. Hier ist schwarz die Paretofront der konstanten Profilierung dargestellt. Die roten Symbole repräsentieren die Paretofront der symmetrischen Profilierung. Im letzteren Fall ist die Quintic-Funktion gewählt worden, deren einzige geometrische Beschränkung darin besteht, daß die Parameterlinien symmetrisch zur Tragflügelmitte sind. Wir werden im folgenden noch sehen, daß diese Wegweisung für die Optimierung keinen gravierend negativen Eingriff darstellt, sondern vielmehr eine durch den GA bevorzugte Parameterlinienführung ist.

Die Paretofronten zeigen keine Auffälligkeiten, die zur Annahme führen könnten, daß der GA nicht sauber auskonvergiert ist. Daß die Paretofront bei geringen Widerstandsbeiwerten abbricht, ist ein Effekt, den man schon für die zweidimensionale Profiloptimierung beobachten konnte. Für die Tragflügeloptimierung scheint dieser Effekt etwas stärker zu Tage zu treten. 


\section{Paretofront}

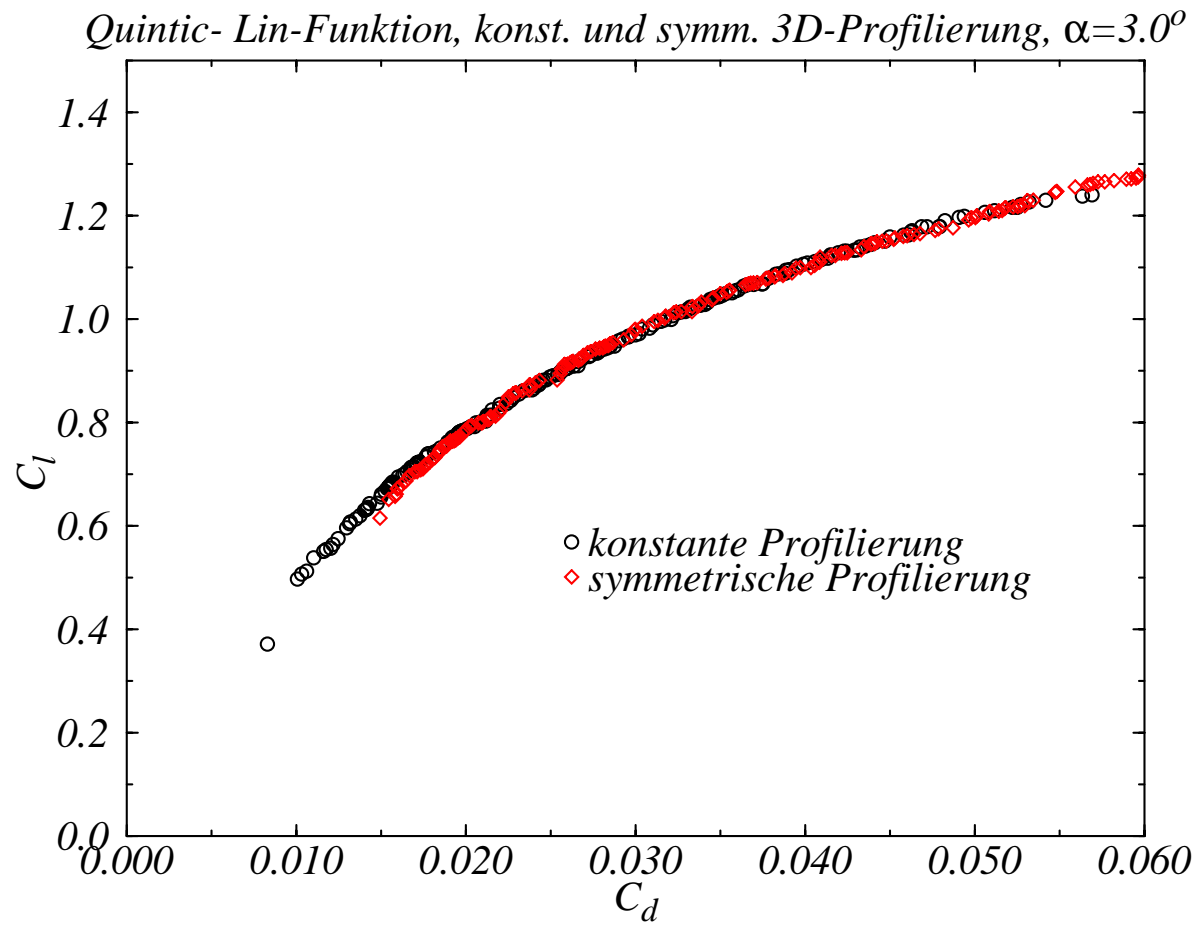

Abb. 35 Quintic- und konstant-lineare-Funktion für die Profilierung. $M_{\infty}=0.707$.

\section{Cp vs X}

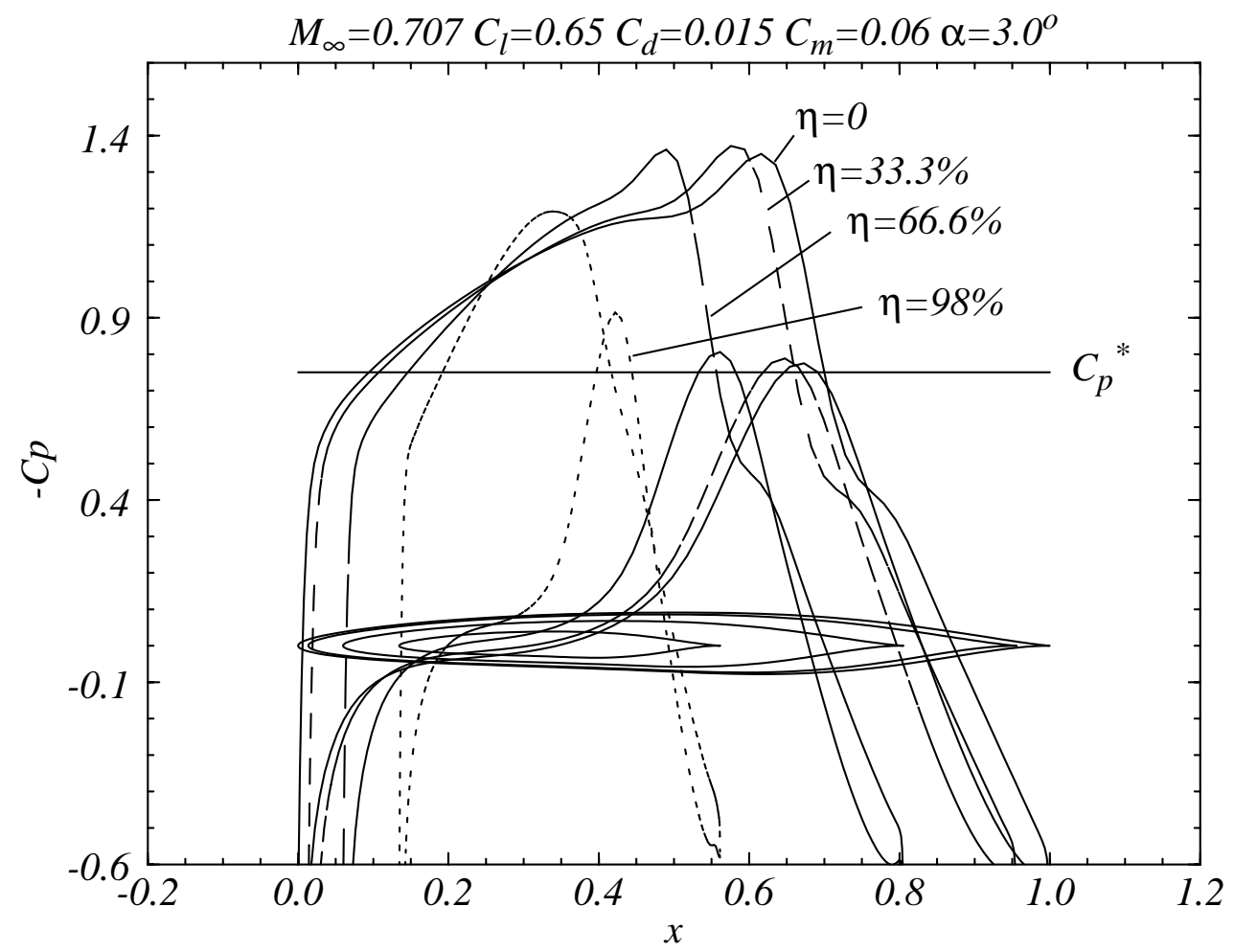

Abb. $36 \mathrm{C}_{\mathrm{p}}$-Verteilung für die spannweitig symmetrische Profilierung. $M_{\infty}=0.707$. 
Die Abb. 36 zeigt das enttäuschende Ergebnis der $\mathrm{C}_{\mathrm{p}}$-Verteilung aus der symmetrischen Profilierung des Tragflügels. Das Ergebnis aus der konstanten Profilierung liegt zwar für die reibungsfreien Auftriebs- und Widerstandsbeiwerte besser, zeigt aber keine Druckverteilung, die einer nachträglichen, reibungsbehafteten Rechnung standhalten würde. Die Grafik sei dem Leser hier erspart.

Welches sind nun die Gründe, für dieses schlechte Ergebnis der Profilierung des Tragflügels? Wie oben schon erwähnt, ist der Paretofront keine Fehlerhaftigkeit zu entnehmen. Der Algorithmus wurde genauso durchgeführt, wie für die zweidimensionale Problematik, die sehr gute Ergebnisse geliefert hatte. Aus diesem Grunde ist das Ergebnis auf die Geometrie selbst oder die strömungstechnischen Parameter zurückzuführen. Die auffälligste vorgenommene Änderung an den strömungstechnischen Parametern ist der Anstellwinkel. Dieser wurde von $\alpha=1.46^{\circ}$ auf $\alpha=3.0^{\circ}$ Grad gesetzt, um einen vergleichbaren Auftriebsbeiwert $C_{1}$ zu erhalten. Dadurch waren die Strömungsfelder der Profile mit dem Strömungsfeld des Tragflügels im mittleren Querschnitt grob vergleichbar. Setzt man versuchsweise den Anstellwinkel auf den Wert von $\alpha=1.46^{\circ}$ zurück und wiederholt die Rechnung oben, erhalten wir folgendes Ergebnis. Hierbei werden die Parameterlinien nicht auf eine Symmetrie zur Tragflügelmitte eingeschränkt. In diesem Beispiel sind die Parameterlinien frei von symmetrischen Zwängen.

\section{Pareto-Front}

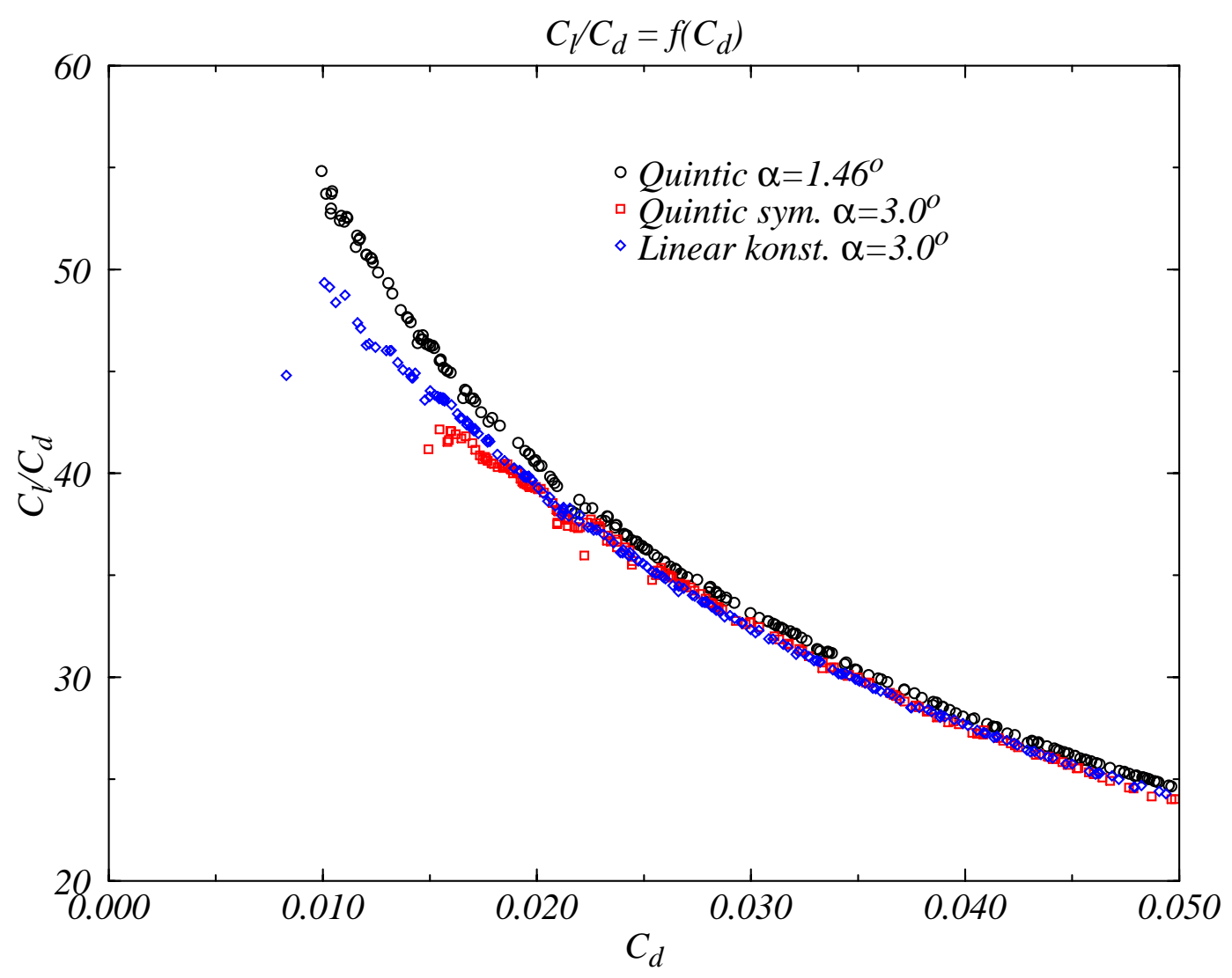

Abb. 37 Quintic- und Lineare-Funktion für die Profilierung. $M_{\infty}=0.707$.

In dem Vergleich der Paretofronten liegt die Paretofront des kleinsten Anstellwinkels am be- 
sten. Dieses ist die Kurve mit schwarzen Kreisen in Abb. 37. Die beiden anderen Paretofronten entsprechen den Kurven in Abb. 35. Hier ist allerdings die Darstellung der Gleitzahl in Abhängigkeit des Widerstandes gewählt worden. Die Überlegenheit der Paretofront des kleinsten Anstellwinkels tritt besonders bei kleinen Widerstandsbeiwerten hervor. Dieser Sachverhalt ist aber über den gesamten Verlauf der Paretofront gegeben. Insgesamt liegt das aerodynamische Optimum besser als das der Paretofronten mit größerem Anstellwinkel. Die Cp-Verteilung im aerodynamischen Optimum der Quintic-Funktion des Anstellwinkels ist in folgender Abbildung dargestellt.

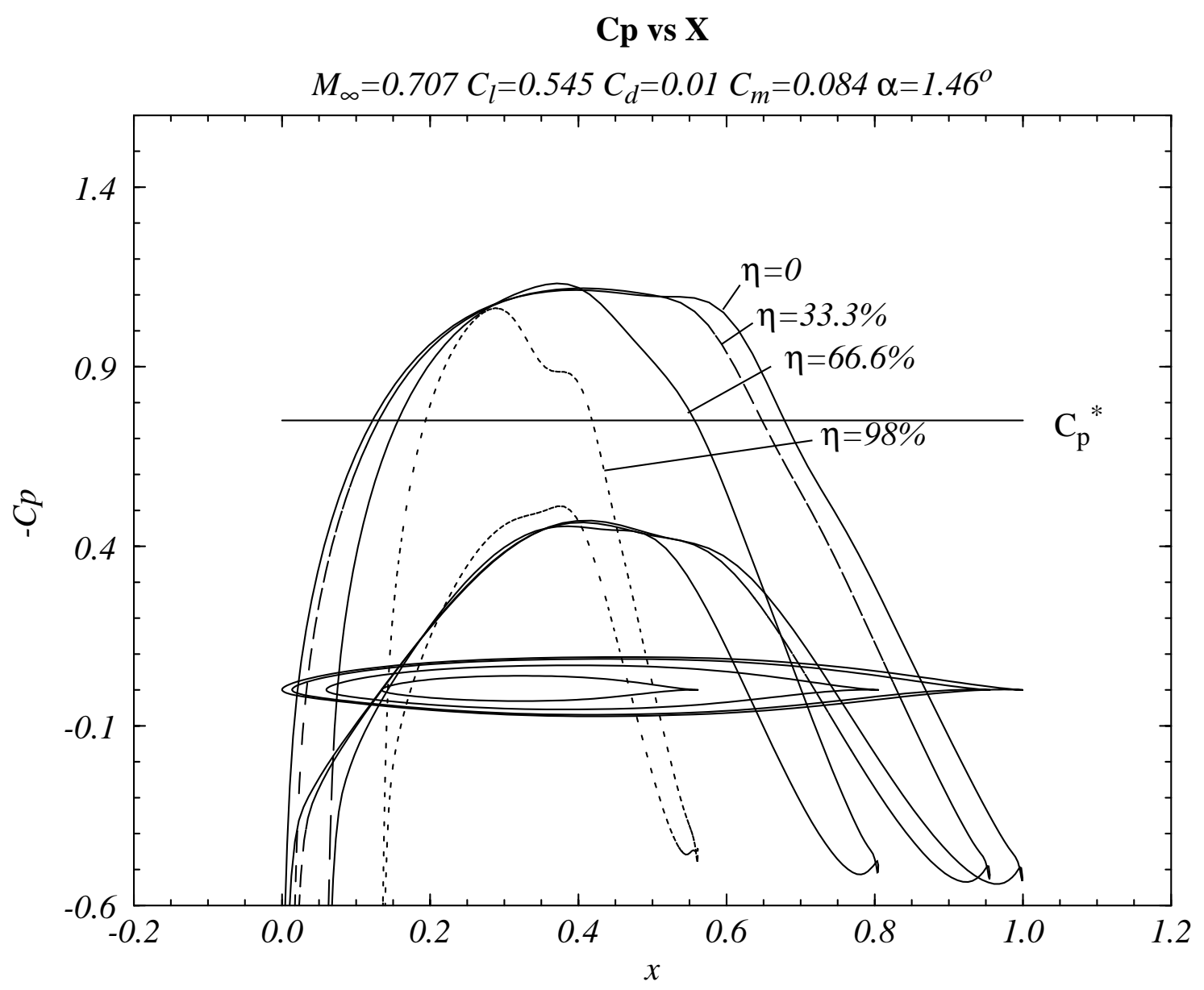

Abb. $38 C_{p}$-Verteilung der Profilierung durch Quintic-Funktion. $M_{\infty}=0.707, \alpha=1.46^{o}$

Offensichtlich ist die Verringerung des Anstellwinkels hier des Rätsels Lösung. Die guten Ergebnisse aus der Profiloptimierung können hier für die Tragflügeloptimierung ebenfalls gefunden werden. Die $\mathrm{C}_{\mathrm{p}}$-Verteilung für den Tragflügel zeigt über die gesamte Spannweite eine stoßfreie Rekompression des Überschallgebietes. Die Abbildungen Abb. 38 bis Abb. 40 zeigen dieses Ergebnis in verschiedener Darstellung. Die $\mathrm{C}_{\mathrm{p}}$-Verteilung zu diesem Tragflügel findet sich in Abb. 38 und Abb. 40. Die Abb. 39 zeigt das lokale Überschallgebiet auf dem Tragflügel.

An dieser Stelle zeigt sich, daß die Stoßfreiheit des Tragflügels direkt mit der geometrischen Definition und den strömungstechnischen Daten zusammenhängt. Die minimale Veränderung 


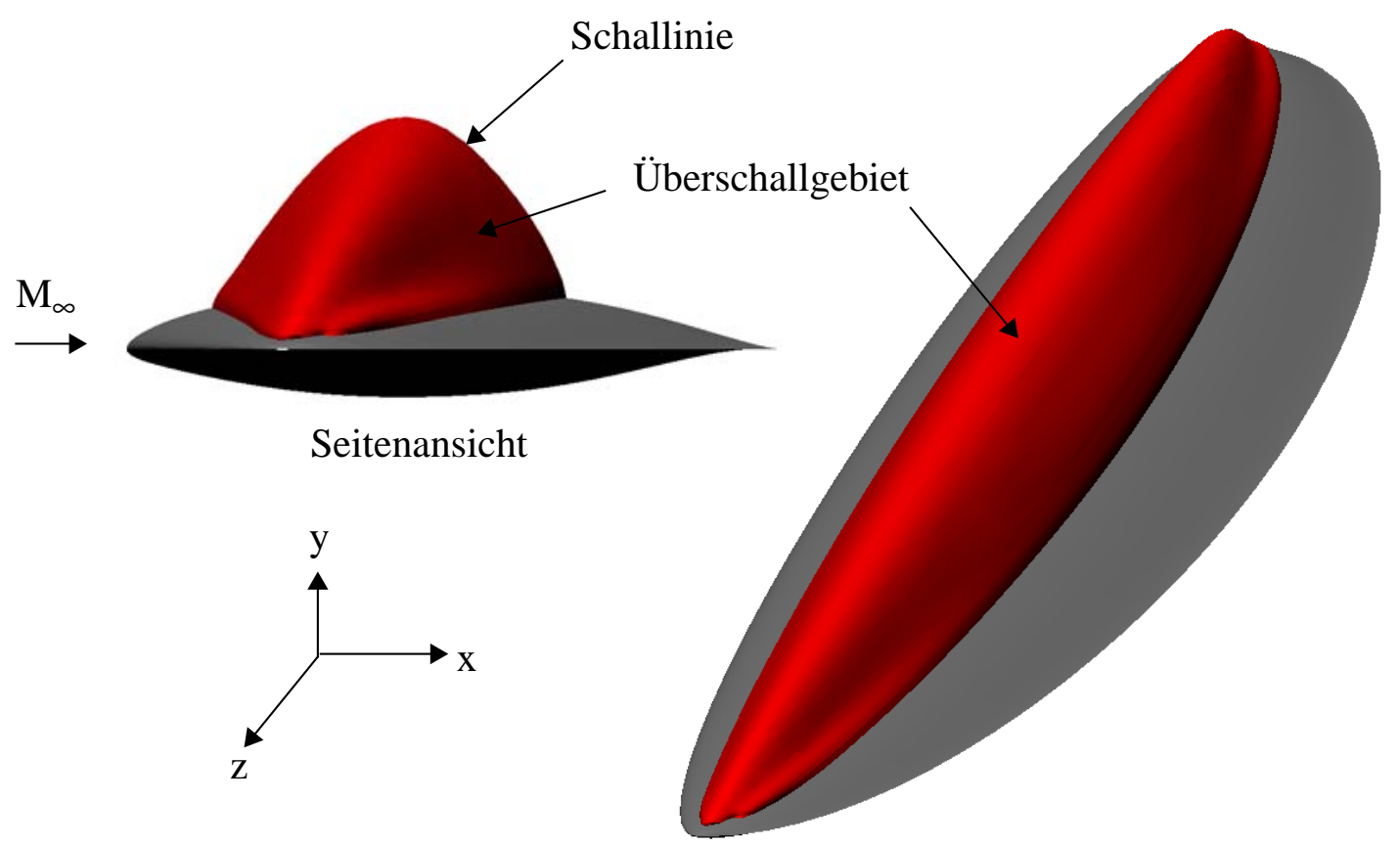

Abb. 39 Isofläche des lokalen Überschallgebietes bei $M=1 . M_{\infty}=0.707, \alpha=1.46^{\circ}, C_{l}=0.545$.

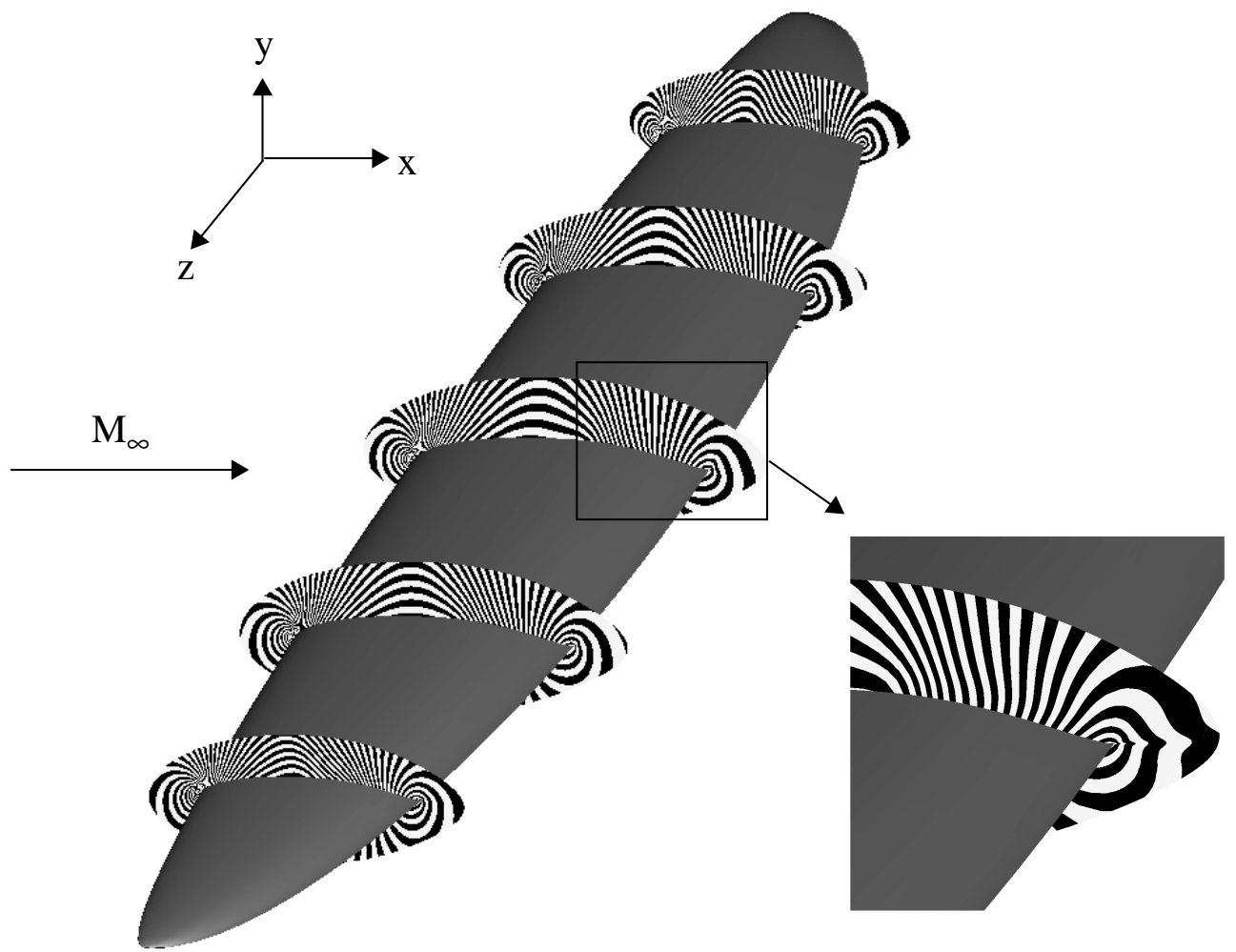

Abb. $40 \mathrm{C}_{\mathrm{p}}$-Verteilung in schwarz-weiß Darstellung an Querschnitten des Tragflügels. 
des Anstellwinkels bringt sofort eine enorme Leistungssteigerung des Tragflügels. Die detaillierte Sichtung der Druckverteilung in Abb. 38 schließt nahtlos an die guten Ergebnisse der Profiloptimierung an. Die Optimierung eines kompletten Tragflügels ist prinzipiell ungleich schwieriger als die Optimierung eines Profils, weil auch dreidimensionale Effekte zu berücksichtigen sind. Die Methode, eine optimale Anpassung der Konfiguration an die Strömungsverhältnisse durchzuführen, ist dieselbe geblieben. Allein die geometrische Definiton ist gegenüber zweidimensionalen Konfigurationen unterschiedlich. Die Geometriedefinition muß an die Optimierungsmethode (dem GA) gut angepaßt sein. Das Einhalten allein dieser Rahmenbedingung liefert offensichtlich gute Ergebnisse.

Die Abb. 39 zeigt das lokale Überschallgebiet des Tragflügels. Hier ist die Seitenansicht, innerhalb der Abbildung, mit der Abb. 10 in 5.1 vergleichbar. Der dort beschriebene ungünstige Effekt des Verdichtungsstoßes konnte hier durch geschickte Manipulation der Geometrie vollständig vermieden werden. Ein Verdichtungsstoß wäre mit einer unstetigen Druckverteilung verbunden. Daß das nicht der Fall ist, ist in Abb. 40 an der $\mathrm{C}_{\mathrm{p}}$-Verteilung im Feld zu sehen. Hier ist über die Spannweite in diskreten Abschnitten die Druckverteilung im schwarz-weißen Muster zu sehen. Die schwarz-weiß-Codierung trägt hier mehr zur guten Visualisierung bei als eine große Farbpalette, denn so ist deutlich zu sehen, daß die Druckverteilung auf dem Tragflügel an der sonst kritischen Stelle des Verdichtungsstoßes sehr regelmäßig ist. Eine Störung ist nicht auszumachen. Diese sehr gute Druckverteilung findet sich auf dem Tragflügel über die gesamte Spannweite.

\section{Auftriebsverteilung}

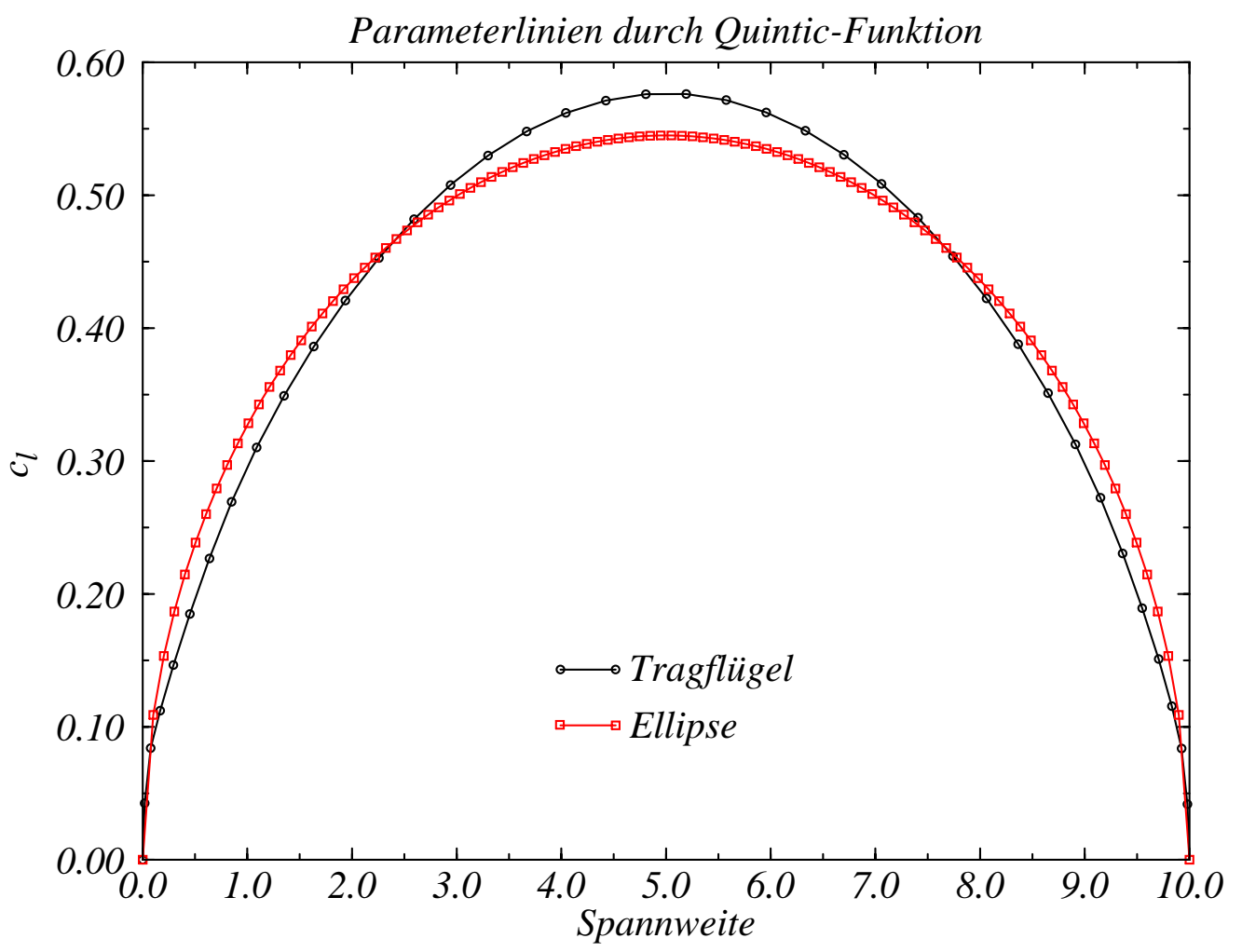

Abb. 41 Auftriebsverteilung des Tragflügels. 
Abb. 41 zeigt die lokalen Auftriebsbeiwerte über die Spannweite. In 7.1 ist bereits beschrieben, weshalb eine elliptische Auftriebsverteilung theoretisch am günstigsten ist. Die Auftriebsverteilung des optimalen Tragflügels kann hier anhand dieser Abbildung überprüft werden. Die Kurve, die mit den schwarzen Symbolen gekennzeichnet ist, ist die errechnete Auftriebsverteilung. Um die Abweichung von der optimalen elliptischen Auftriebsverteilung bestimmen zu können, ist zusätzlich eine Ellipse (rote Symbole) eingezeichnet, die im Scheitelpunkt den Wert des Gesamtauftriebsbeiwertes $C_{l} \approx 0.545$ hat. Es sind zwei wichtige Punkte dieser Abbildung zu entnehmen.

Die Tragflügelkontur ist als elliptischer Grundriß vorgegeben worden. Im Gegensatz zu den Ergebnissen in Abb. 35 und Abb. 36 ist jedoch die Profilierung spannweitig keiner Beschränkung unterlegen. In einem ersten Versuch wurde die Profilierung zunächst als konstant, dann als symmetrisch vorgegeben. Beide Ergebnisse waren unbefriedigend. In Abb. 38 ist keine Zwangsbedingung vorgegeben worden. Dennoch hat sich eine symmetrische Profilierung eingestellt, mit dem Scheitelpunkt in der Tragflügelmitte. Für die Profilierung wäre ohne Zwangsbedingung auch jede andere Form der Auftriebsverteilung möglich gewesen. Aber tatsächlich stellt sich nahezu eine perfekte Ellipse als Auftriebsverteilung ein. Durch den Grundriß des Tragflügels bietet sich eine elliptische Auftriebsverteilung zwar an, ist aber aufgrund der Freiheitsgrade der Profilierung keineswegs zwingend. Damit bestätigt das Ergebnis die klassische Theorie hinsichtlich der elliptischen Auftriebsverteilung.

Die klassische Theorie gilt für inkompressible Strömungen. Die hier vorliegenden Rechnungen wurden mit den Eulergleichungen durchgeführt, die kompressible Effekte berücksichtigen. Deshalb liegt es nahe, daß die Abweichung der Auftriebsverteilung in Abb. 41 von der perfekten Ellipse (rote Symbole) auf diesen Sachverhalt zurückzuführen ist. Die kompressiblen Effekte spielen bei den hier gewählten Strömungskennzahlen eine Rolle. Die Differenz zur klassischen linearen Theorie liegt allem Anschein nach in dieser Tatsache begründet.

Im weiteren Verlauf der Paretofront der letzten Rechnung ist ein Effekt zu beobachten (hier nicht dargestellt), wie er schon in Abb. 21 für die Profiloptimierung zu sehen war. Ab einem bestimmten Punkt auf der Paretofront bei erhöhtem $C_{1}$ kann die Geometrie nicht mehr stoßfrei angepaßt, und so der Verdichtungsstoß auf der Profiloberhälfte nicht mehr vermieden werden. Im Fall für den Tragflügel ist etwas ähnliches sichtbar. Auch hier ist die Geometrie ab einem bestimmten $\mathrm{C}_{1}$ nicht mehr in der Lage, alle ungünstigen Effekte zu vermeiden. Von den Tragflügelenden her breitet sich zunehmend ein Verdichtungsstoß in Richtung Tragflügelmitte aus. Siehe Abb. 42. Der optimale Zustand der Druckverhältnisse auf dem gesamten Flügel erscheint ein Kompromiß zwischen Tragflügelmitte und Tragflügelenden zu sein. Ist die Geometrie nicht mehr im Stande, auf ungünstige Strömungsverhältnisse zu reagieren, wird dort die geometrische Nachführung vernachlässigt, wo sie strömungstechnisch am wenigsten ins Gewicht fällt. Das ist an den Tragflügelenden.

Dieser Sachverhalt ist mit der einsektionalen Geometriedefinition in Spannweitenrichtung durch die Quintic-Funktion zu erklären. Die Parameterlinien passen sich, wie oben schon festgestellt, symmetrisch zur Tragflügelmitte an. Wegen der Reduktion des induzierten Widerstandes ist das sinnvoll. Aber diese Einsektionalität der Parameterlinien erscheint für höhere Auftriebsbeiwerte hinderlich zu sein. Aus Abb. 42 ist herauszulesen, daß eine mehrfache Unterteilung der Parameterlinien in Spannweitenrichtung von Vorteil sein könnte. Dann wäre eine 
Korrektur an den Tragflügelenden möglich, ohne die günstigen Strömungsverhältnisse in der Tragflügelmitte negativ zu beeinflussen. Um also die Leistungsfähigkeit des Tragflügels weiter zu verbessern, könnte man an dieser Stelle die geometrische Definition erweitern. Dieser Schritt

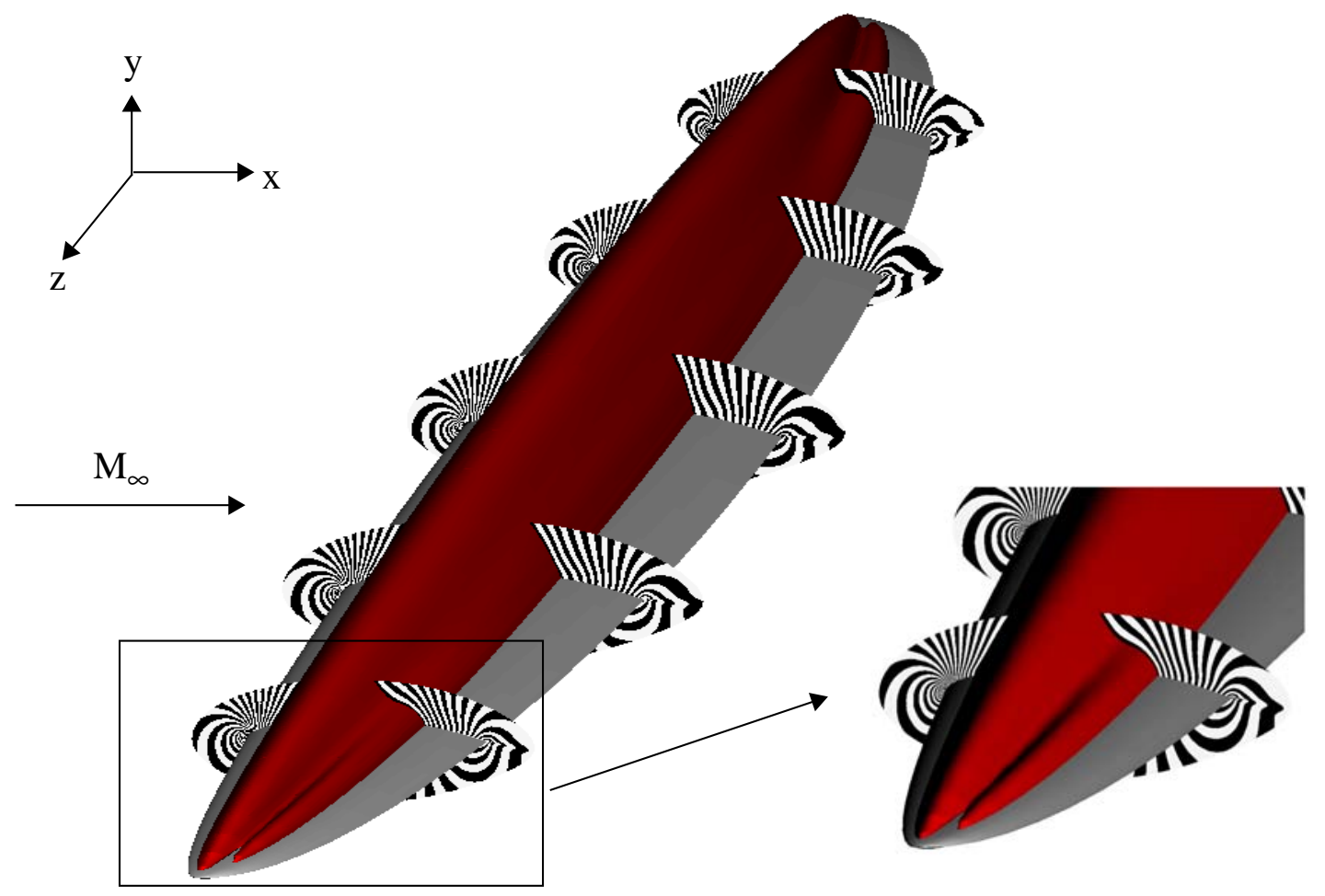
Abb. 42 Machzahlverteilung mit Isofläche $M=1.0 . M_{\infty}=0.707, \alpha=1.46^{0}, C_{l}=0.79$. Ausbreitung des Verdichtungsstosses an den Tragflügelenden.

ist in dieser Arbeit nicht vollzogen. Solch eine Erweiterung wird aber für praktische Anwendungen noch von Bedeutung sein.

Eine Sichtung der Parameterlinien bringt keine anderen Erkenntnisse, als die Auswertung der Parameter im Fall der Profiloptimierung. Bei der Profiloptimierung konnte durch Sichtung der Streuung der Parameter auf die geometrische Abhängigkeit charakteristischer Profilbereiche geschlossen werden. Die charakteristischen Eigenschaften und Einflußbereich der Geometrie wurden durch die Rechnungen bestätigt. Auch hier findet sich die Abhängigkeit dieser Eigenschaften wieder. 


\section{Ergebnisse für Tragflügelkonfigurationen im Überschall}

Es ist von Vorteil hier zunächst grundsätzliche theoretische Betrachtungen zu berücksichtigen, die einen Hinweis auf die günstigste geometrische Form eines Überschallverkehrsflugzeugs geben können. Nicht unbeachtet sollen hier reale Anforderungen bleiben, die von Ingenieuren aus der heutigen Flugzeugindustrie gestellt werden. Die Anforderungen an ein Überschallverkehrsflugzeug sind in der Regel widersprüchlich. Dennoch bleiben einige grundsätzliche Anforderungen bestehen.

- Für eine hohe Leistung im Überschallflug muß die Konfiguration sehr schlank sein, sollte scharfe Überschallvorderkanten besitzen und eine limitierte Spannweite mit sehr dünnem Flügel haben. Die Geometrie selbst sollte variabel sein. Die Triebwerke sollten einen geringen Durchmesser haben.

- Für reduzierten Lärm beim Start muß die Konfiguration eine große Spannweite aufweisen, gerundete Vorderkanten besitzen, und die Triebwerke sollten einen großen Durchmesser haben.

Der Widerspruch in der Konfigurationsauslegung ist wesentlich auf die unterschiedlichen physikalischen Gesetzmäßigkeiten von Unter- und Überschallströmungen zurückzuführen. Die Widersprüche sind quasi naturgemäß gegeben, da jedes Überschallverkehrsflugzeug (bei Start, Landung, Überlandreiseflug) erst den Unterschallbereich überbrücken muß. Die Gesetzgebung vieler Länder verbietet tatsächlich den Überschallflug innerhalb der Hoheitsgrenzen. Deswegen wird sich der Überschallverkehrsflug vorwiegend auf die Überquerung der Ozeane konzentrieren. Man rechnet grob, daß 2/3 der Reiseflugzeit dann im Überschall geflogen werden kann, während 1/3 der Reiseflugzeit für den Überlandflug bleibt. Ein wirtschaftliches Verkehrsflugzeug muß also beiden Geschwindigkeitsbereichen Rechnung tragen. Das stellt die Ingenieure vor eine widersprüchliche Aufgabenstellung.

\subsection{Allgemeine Betrachtungen zur Auslegung einer Überschallkonfiguration}

Bei der Luftfahrtindustrie [8], S.66 ff., hat man dazu folgende grundsätzliche Konfigurationen betrachtet, die hinsichtlich ihrer Eignung für eine Überschallkonfiguration geprüft wurden.
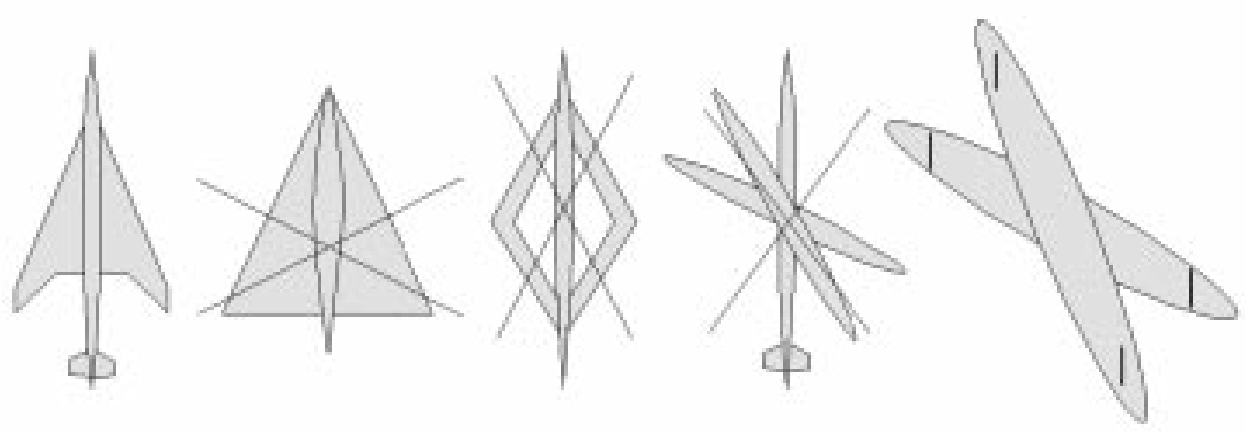

Abb. 43 Die Auswahl einer wirtschaftlich überlebensfähigen Überschallkonfiguration. 
Die Deltaflügel-Rumpfkonfiguration scheidet wegen zu geringer „Schlankheit“ aus. Sämtliche Nutzlast ist im Rumpf konzentriert. Der Wellenwiderstand im Überschall wäre zu hoch. Die Rautenflügelkonfiguration hat nur begrenzte Spannweite. Das Tankvolumen wäre hier drastisch reduziert, was eine geringe Reichweite zur Folge hätte. Weiter sind strukturelle Probleme zu erwarten. Die schräge Flügel-Rumpfkombination ist ausschließlich für kleine Flugzeuge bei kleiner Überschallmachzahl interessant. Es verbleiben die beiden äußeren Konfigurationen: links, wie sie heute in der Concorde existiert, und rechts die neuartigste Konfiguration, welche als OFW (Oblique Flying Wing) steigendes Interesse findet. Die letztgenannte Konfiguration ist hier die einzige Konfiguration, die eine Geometrievariation für Unter- und Überschallflug durch variable Pfeilung, bzw. Schrägstellung der Gesamtkonfiguration erreicht.

\subsection{Auslegung einer Überschallkonfiguration nach der linearen Theorie}

R.T.Jones gibt in [17] einen Vergleich des theoretischen Widerstandes für einen schrägen elliptischen Flügel und einen Deltaflügel. Dieses sind die beiden Konfigurationen, die nach den eben gemachten Überlegungen für einen ersten Überschallentwurf in Frage kommen.

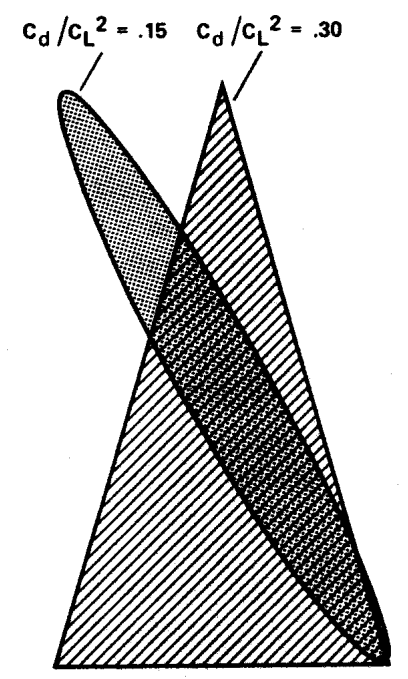

\section{Abb. 44 Vergleich des theoretischen Widerstandes eines elliptischen Flügels und eines Deltaflügels nach R.T. Jones.}

Demnach stellt zumindest theoretisch der OFW hinsichtlich der Wirtschaftlichkeit die bessere Konfiguration dar.

Welche prinzipielle Aussagen kann man über den Zusammenhang der geometrischen Form eines Überschallflugkörpers, und dessen Widerstand und Auftrieb, machen? Betrachten wir zunächst den Widerstand. Im Gegensatz zu einem Körper in Unterschallanströmung erfährt ein Körper in Überschallanströmung neben Druck-, Reibungs- und induzierten Widerstand einen zusätzlichen Widerstand, den sog. Wellenwiderstand. Der Gesamtwiderstand berechnet sich bei gegebenen Auftrieb nach [8] als Minimum des Widerstandes aus der linearen Theorie zu: 


$$
D=q \cdot S_{f} \cdot C_{f}+\frac{L^{2}}{\pi \cdot q \cdot s^{2}}+\frac{\beta^{2} \cdot L^{2}}{\pi \cdot q \cdot l_{l}^{2}}+\frac{128 \cdot q \cdot V^{2}}{\pi \cdot l_{v}^{4}}
$$

Der Widerstand setzt sich hiernach aus vier Komponenten additiv zusammen. Hierbei ist der erste Term der Reibungswiderstand, der zweite Term der induzierte Widerstand, der dritte Term der Wellenwiderstand in Folge von Auftrieb, der vierte Term der Wellenwiderstand in Folge von Volumen. Die Größen im Einzelnen:

• $q$ - dynamischer Druck,

• $S_{f}$ - Referenzfläche für Oberflächenreibung,

- $C_{f}$ - Reibungsbeiwert,

$\bullet L$ - Auftrieb,

•• $s$ - die Größe $L^{2} / \pi q_{n} l_{l}^{2}$ sei der induzierte Widerstand normal zur Strömung und $b$ die Spannweite des nichtgepfeilten Flügels. Man interpretiert dann das Produkt $q_{n} b^{2}$ als $q \cos ^{2} \lambda b^{2}$ oder $q s^{2}$. Mit $\lambda$ Pfeilungswinkel und $s$ der Spannweite normal zur freien Anströmung.

•• $V$ - Volumen des Tragflügels, und $\beta=M^{2}-1$.

•• $l_{v^{-}}$Gesamtlänge des Flugkörpers,

• $l_{l}$ - Auftriebserzeugende Länge des Flugkörpers.

Die Voraussetzung für die Gültigkeit der oben angegebenen Formel ist eine elliptische Auftriebsverteilung.

Welche Rückschlüsse auf die geometrische Form eines Körpers in Überschallanströmung lassen sich nach oben angegebener Formel ziehen? Zur Vereinfachung vernachlässige ich hier zunächst den Reibungswiderstand. Die Berechnung des Reibungswiderstandes erfordert Lösungen der Navier-Stokes-Gleichungen, die den Rechenaufwand für einen GA hier erheblich in die Höhe treiben würden. Da fast alle hier vorgestellten Rechnungen Lösungen der EulerGleichungen sind, sei dies als vorläufige Einschränkung gestattet. Die Verfahrensweise der Optimierung mit einem GA ändert sich nicht, wenn man zusätzlich auch den viskosen Widerstand betrachtet. Da ein Entwurfsverfahren aber in endlicher Zeit auch einen Entwurf liefern sollte, vermeiden wir hier die zusätzliche Berechnung des Reibungswiderstandes und beschränken uns auf ein Ergebnis, welches die Rechenzeit in Grenzen hält.

Der verbleibende Widerstand in der Formel (18) ist der induzierte Widerstand und der Wellenwiderstand in Folge von Volumen und Auftrieb. Wie lassen sich nun diese drei Arten von Widerstand gleichzeitig reduzieren?

Der induzierte Widerstand ist vor allem gekennzeichnet durch das Seitenverhältnis der Tragfläche, welches hier im Nenner quadratisch auftritt. Bei vorhandenem Schiebewinkel ist diese Länge die Projektion auf die senkrecht zur Strömungsebene liegende Ebene. Ist die Auftriebs- 
verteilung in einer Ebene, z.B. in der Spannweitenebene, elliptisch und hat der Tragflügel einen elliptischen Grundriß, so ist die Auftriebsverteilung in jeder Azimutalebene auch elliptisch. Friert man die Schnittebenen ein, um den Wellenwiderstand in Folge von Volumen zu reduzieren, verbleiben drei Möglichkeiten, um eine elliptische Auftriebsverteilung zu erhalten: Variation der Biegung des Tragflügels, Variation der Wölbung des Tragflügels und Verdrehen des Tragflügels. Genau dieses sind die eingangs dieses Kapitels beschriebenen Möglichkeiten der Geometrievariationen für den GA.

Um gleichzeitig den Wellenwiderstand in Folge von Volumen minimal zu halten, sind die Schnittebenen einzufrieren. Nach der Supersonic-Area-Rule ist der Wellenwiderstand in Folge von Volumen dann minimal, wenn der äquivalente Rotationskörper einer Sears-Haack-BodyVerteilung entspricht. Dazu ist die Machfläche mit dem Körper zu verschneiden und über eine volle Rotation um die Körperachse auf die zur Strömungsebene senkrechte Ebene zu projizieren und dann zu mitteln. Dieser Vorgang ist über die gesamte Länge des Flugkörpers zu wiederholen. Ist die resultierende Kurve proportional zu der von Sears und Haack angegebenen, ist der Wellenwiderstand in Folge von Volumen nach dieser Regel minimal.

Als letztes ist der Widerstand in Folge auf Auftrieb zu minimieren. In (18) steht hier die Länge der Traglinie im Nenner. D.h. sie ist möglichst groß zu wählen, was wiederum bedeutet, daß nach Möglichkeit der Flugkörper über seine volle Länge auftriebserzeugend sein sollte.

Ein gepfeilter elliptischer Flügel verbindet große Spannweite mit großer Traglinie. Die Reduktion des Wellenwiderstandes eines schräg fliegenden Flügels rührt von seiner Fähigkeit her in jeder azimutalen Ebene die optimale Verteilung von Auftrieb und Volumen zu liefern. Der aerodynamische Vorteil eines schräg fliegenden Flügels gegenüber einem gepfeilten Flügel bei supersonischen Machzahlen beruht vor allem auf dieser Tatsache.

\subsection{Beispiel einer Konfiguration aus der klassischen linearen Überschallheorie}

Aus den oben genannten Betrachtungen läßt sich theoretisch auch ein optimaler OFW entwerfen, wenn man die dazu notwendigen Techniken der Geometriedefinition besitzt. Tatsächlich ist der geometrische Entwurf und die flexible Handhabung der Geometriewerkzeuge hier von zentraler Bedeutung, denn jedes strömungsmechanische Problem und dessen Lösung ist über die Strömungsgrößen auch eng mit der umströmten Geometrie selbst verknüpft. Die oben angegebene Formel (18) gibt hier wesentliche Hinweise zur Konfigurationsauslegung.

In der Literatur findet sich in [42] ein OFW, welcher das Ergebnis von Überlegungen zu dieser Thematik unter den oben genannten Randbedingungen ist und vollständig mittels der klassischen linearen Überschalltheorie entworfen wurde. Welche Eigenschaften kennzeichnen dieses Entwurfsbeispiel?

Die Konfigurationsauslegung dieses OFW ist der Versuch, den Widerstand nach (18) in jeder Hinsicht durch eine sukzessive manuelle Vorgehensweise zu minimieren. Um diesen Sachverhalt zu verdeutlichen, siehe Abb. 45 a). Diese Abbildung ist direkt auf Abb. 45 c) bezogen. Die Anströmung erfolgt mit der Machzahl $M_{\infty}=1.414$ unter einem Schiebewinkel von $60^{\circ}$. Diese Hauptanströmungsrichtung wurde in zwei Komponenten zerlegt: in eine senkrechte Anströ- 
mung mit $M_{n}=0.707$, und eine parallele Anströmung mit der Machzahl $M_{t}=1.23$. Für beide Anströmungskomponenten wurde der Widerstand minimiert.

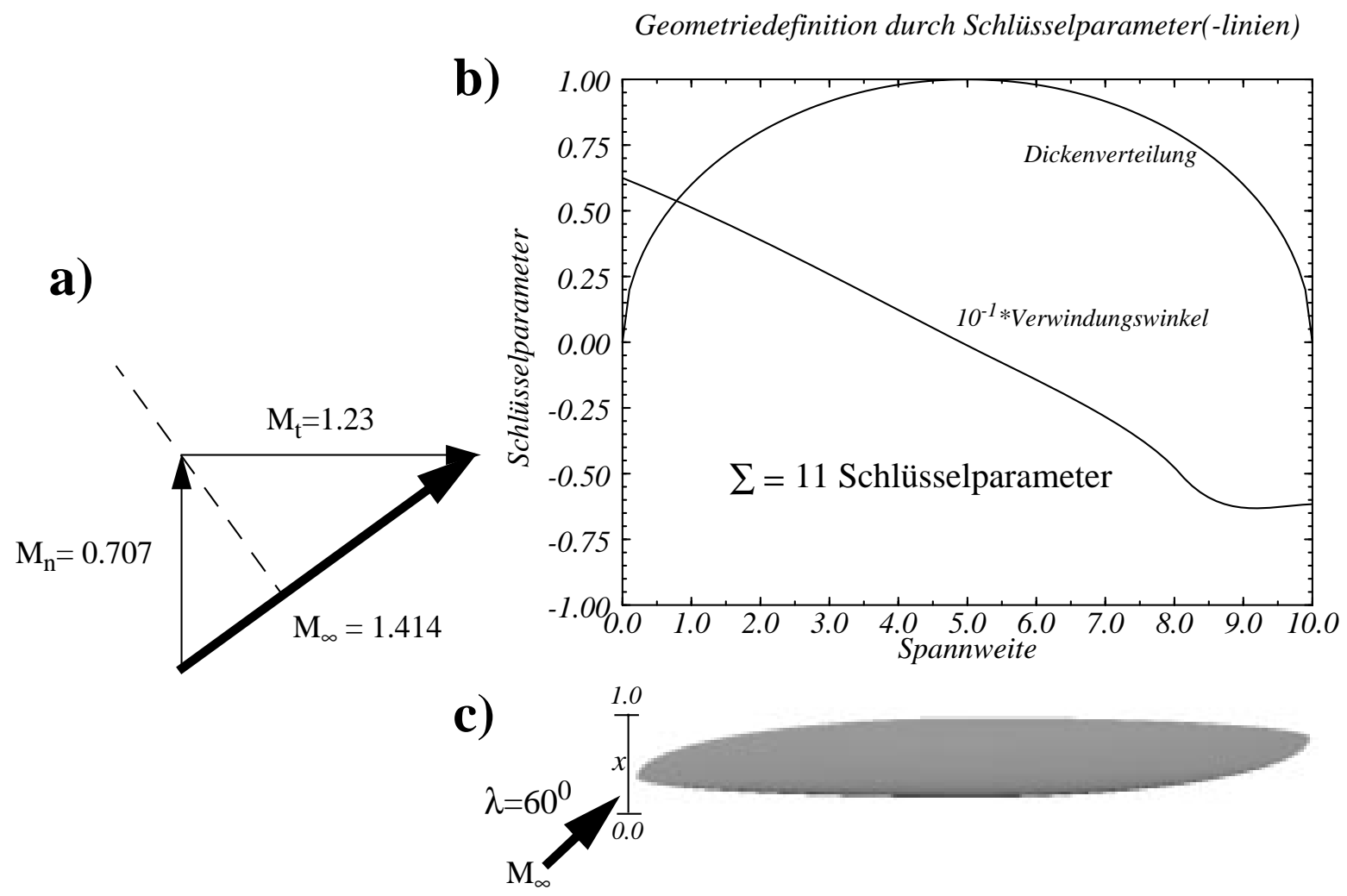

\section{Abb. 45 Oblique Flying Wing in supersonischer Anströmung.}

Die senkrechte Anströmung ist eine transsonische Unterschallanströmung. Zu dieser Machzahl existieren hinreichende Erkenntnisse zur Flügelauslegung (siehe die vorherigen Kapitel). Die Widerstandsminderung bei dieser Machzahl geschieht vorwiegend durch stoßfreie Auslegung. Dieser Schritt ist schon in der vorliegenden Arbeit vollzogen worden. Eine stoßfreie Konfiguration könnte auch aus der Paretofront vorangegangener Kapitel, der Profilierung oder der Tragflügelauslegung entnommen werden. Somit erhält man eine optimale Konfiguration hinsichtlich dieser Anströmungsbedingung.

Die tangentiale Komponente der Anströmung liegt im Überschallbereich. Die Widerstandminimierung geschieht hier durch Modellierung nach der Supersonic-Area-Rule [17]. Diese bezieht sich hier auf die Volumenverteilung eines Flugkörpers in Überschallanströmung. Die geometrische Auslegung des hier beschriebenen Beispiels hält diese Regel durch die elliptische Planform, mit einer elliptischen Dickenverteilung über die Spannweite, ein. Durch diese flexible geometrische Formgebung läßt sich der Wellenwiderstand in Folge von Volumen theoretisch auf das Minimum nach der linearen Theorie reduzieren. Die geometrische Gestaltung ist wie in Abb. 45 b) und c) sichtbar.

In einem letzten Schritt wird noch der günstige induzierte Widerstand mit einer geeigneten Variation des Verdrehungswinkels über die Spannweite realisiert. Die Verteilung dieses Winkels 
wurde so gewählt, daß sich in der Projektionsebene senkrecht zur realen Anströmung eine elliptische Auftriebsverteilung einstellt. Damit ist dann der induzierte Widerstand des OFW theoretisch auf ein Minimum reduziert.

Der so gestaltete OFW ist ein Beispiel, welches Sobieczky et al. in [42] angibt. Er ist das Produkt manueller Optimierung, wie oben beschrieben. Das Beispiel ist somit nicht Resultat eines praktischen Entwurfsprogramms, wohl aber ist es geeignet für die Kalibrierung von systematischen, numerischen Optimierungsverfahren, wie der hier vorgestellten Methode.

\subsection{Auslegung einer Überschallkonfiguration durch den Genetischen Algorithmus}

Das theoretische Widerstandsminimum des OFW kann jetzt mit Hilfe eines genetischen Algorithmus überprüft werden. Die Ergebnisse der bisher durchgeführten Rechnungen können jetzt auf die Problematik in einer Überschallanströmung übertragen werden. Der Schwierigkeitsgrad für den GA wird sich damit weiter erhöhen.

Im jetzt folgenden Fall für eine Konfigurationsauslegung im Überschall sollen die Vorgaben aus der Geometriedefinition der Unterschallrechnungen nur grob vorgegeben werden. D.h. die Funktionen, die die Tragflügelplanform sowie die Profilierung definieren, werden jetzt den Fall einer elliptischen Planform bestenfalls als Spezialfall beinhalten, aber ihn nicht notwendig reproduzieren. Als weiterer zusätzlicher offener Parameter wird der Verwindungswinkel zugelassen. Der Verwindungswinkel längs der Spannweite wird allerdings zunächst auf eine lineare Funktion beschränkt. Weiterhin wird jetzt auch keine symmetrische Profilierung mehr vorgegeben, diese Vorgabe erscheint wegen des großen Schiebewinkels von $60^{\circ}$ auch wenig angebracht.

Insgesamt stehen in diesem Abschnitt drei wesentliche Größen zur Optimierung an. Die Planform, der Verwindungswinkel des Tragflügels und die Profilierung längs der Spannweite. Die Planform des Tragflügels muß dann selbst keinen exakt elliptischen Grundriß mehr haben, um die günstige elliptische Auftriebsverteilung zu induzieren. Die lineare Theorie kann hier nur noch sehr grobe Vorgaben machen. Ein GA erscheint hier das geeignete Mittel, trotz der vorherrschenden Nichtlinearitäten dieser Strömungsverhältnisse eine erfolgreiche Optimierung durchzuführen.

Die reale Überschallanströmung eines schräg fliegenden Flügels ruft stark nichtlineare Effekte hervor. Durch den scharfen Verdichtungsstoß vor dem Tragflügel ist hinter diesem Stoß auf dem Tragflügel die Strömung möglicherweise stark gestört. Dieser Sachverhalt wird die Profilierung des Tragflügels erheblich beeinflussen. Die Profilierung wird ebenfalls stark von der Konturlinie der Vorderkante beeinflußt sein. Es ist daher interessant, ob bei dem hohen Anteil von Nichtlinearitäten der GA zu einem mit dem manuell optimierten OFW überhaupt vergleichbaren Resultat gelangt.

In [42] ist der Anteil nichtlinearer Effekte bei zunehmendem Dickenverhältnis des Tragflügels in Abweichung von der linearen Theorie beschrieben. Dieser Anteil steigt bei zunehmendem Dickenverhältnis des Tragflügels stark an, d.h. es ist eine hohe Abweichung von der linearen Theorie bei realen Auslegungen eines schräg fliegenden Flügels zu erwarten. Deswegen ist zu- 
nächst zu vermuten, daß die Werte für Auftriebs- und Widerstandsbeiwert auf der Paretofront besser liegen, als der Wert des OFW aus der klassischen linearen Überschalltheorie.

Diesen Sachverhalt zu überprüfen, ist Ziel der nun folgenden Berechnungen. Die Anströmmachzahl wird auf $M_{\infty}=1.414$ gesetzt, und ein Schiebewinkel von $\lambda=60^{\circ}$ vorgegeben. Die geometrischen Variationsmöglichkeiten sind oben schon beschrieben. Das Ergebnis aus dieser Rechnung ist in Abb. 46 zu sehen. Auch hier beziehen sich die wesentlichen Betrachtungen wieder auf Auftrieb und Widerstand, bzw. auf deren aerodynamische Beiwerte, und das Ergebnis ist wieder in Form einer Paretofront gegeben.

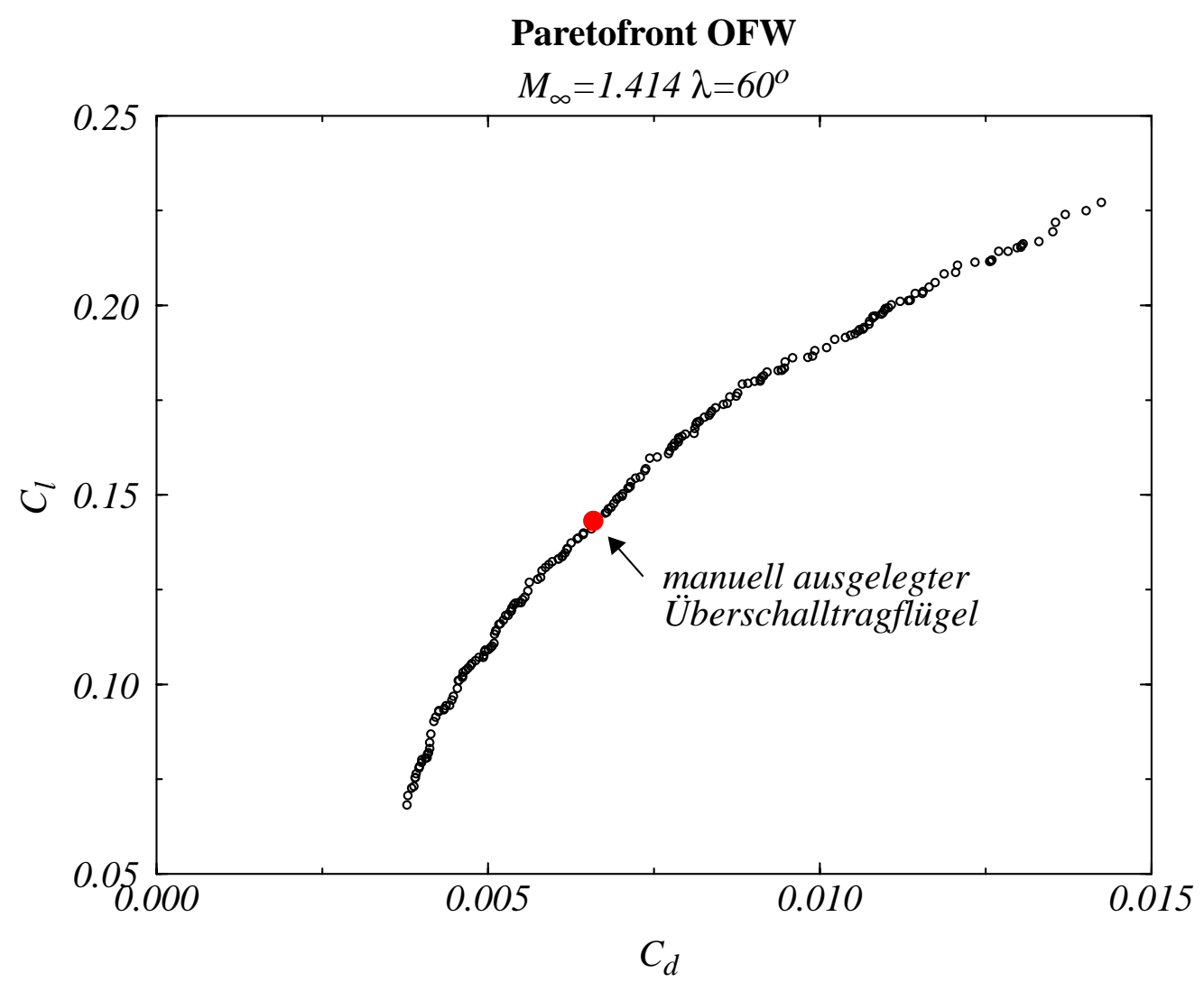

Abb. 46 Paretofront der Überschallrechnung. $M_{\infty}=1.414, \lambda=60^{\circ}$

Diese Abbildung zeigt das maximal mögliche Verhältnis der Auftriebsbeiwerte zu den Widerstandsbeiwerten für den Überschall in Form der Paretofront.

Die Abb. 46 zeigt ein einigermaßen überraschendes Ergebnis. Die Beiwerte des oben beschriebenen OFW aus der klassischen linearen Überschalltheorie sind in dieser Grafik mit einem roten Punkt gekennzeichnet. Dieser liegt direkt auf der Paretofront. Die erwarteten Vermutungen des Abweichens der Resultate für ein Optimum (bei $C_{l} \sim 0.14$ ) treffen nicht in dem Maße zu wie oben beschrieben. Der mit klassischen Mitteln ausgelegte OFW steht mit seinem Leistungsbereich den optimierten vergleichbaren Tragflügeln der Paretofront in nichts nach. Das bedeutet, daß tatsächlich der lineare Ansatz einen optimalen Punkt geometrischer Definition für diese Anströmbedingungen findet, somit die klassischen Methoden im Bereich dieser Anwendungen 
ein sehr brauchbares praktisches Werkzeug darstellen.

Mit der Einschränkung, daß dem GA nicht erlaubt wurde, auch die Planform zu variieren, erreichen Beispiele des GA im Bereich von $C_{l} \sim 0.14$ keine geringeren Werte für $\mathrm{C}_{\mathrm{d}}$ als sie unter Anwendung der klassischen Theorie erreichbar sind.

\section{Paretofront OFW}

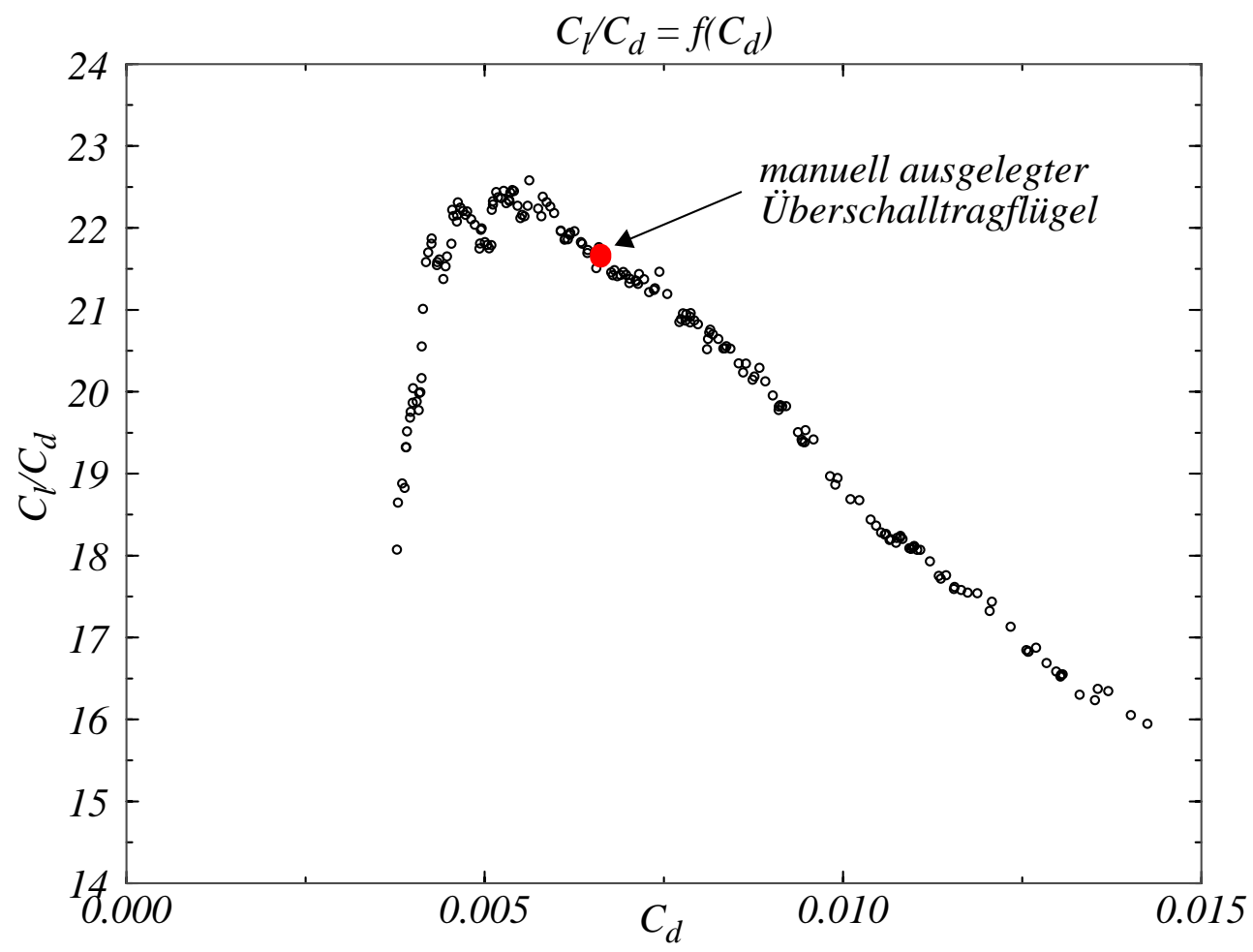

\section{Abb. 47 Paretofront der Überschallrechnung. $M_{\infty}=1.414, \lambda=60^{\circ}$}

Der manuell entworfene OFW liegt jedoch nicht genau im aerodynamischen Optimum, wie die Abb. 47 zeigt. Die Beurteilung, ob dieser OFW überhaupt in der Nähe des erreichbaren aerodynamischen Optimums liegt, ist um Grunde nur durch die Paretofront möglich. Tatsächlich stellt der manuell entworfene OFW den bisher besten Entwurf in diesem Leistungsbereich dar. Ein Maßstab zur Beurteilung der Geometrie war bisher nicht verfügbar. Erst durch die Variation der Geometrie in naher Anlehnung an die geometrische Definition dieses vorgegebenen Beispiels kann ein direkter Vergleich dieses Entwurfs gezogen werden.

Die Abbildung macht deutlich, daß die Möglichkeiten der Geometriedefinition, zur Anpassung an die vorherrschenden Strömungsverhältnisse im Überschall bei diesem Beispiel noch nicht voll ausgeschöpft sind. Der manuell gefundene OFW hat ein Verhältnis von $L / D \approx 21.3$. Das aerodynamische Optimum liegt hier bei $L / D \approx 22.6$. Diese Verbesserung (ca. 6\%) ist durch Ausschöpfen der geometrischen Möglichkeiten der Profilierung und Verwindung, aber noch nicht einmal auch der Planform, erreicht worden. 


\section{Zusammenfassung}

Diese Arbeit stellt eine Anwendung und Erweiterung evolutionärer Methoden auf aerodynamische Optimierung dar. Durch Implementation der natürlichen Anpassungungsmethode der Evolution in einen Computeralgorithmus kann diese Methode auch für die Aerodynamik nutzbar gemacht werden. Bewußt wird hier auf Studien zur Steigerung der evolutionären Konvergenzgeschwindigkeit verzichtet. Dieses ist Gegenstand vieler anderer wissenschaftlicher Arbeiten zu diesem Thema. Im Mittelpunkt steht hier die Geometriedefinition, deren Qualität für die Aerodynamik durch die evolutionären Methoden beurteilt werden kann.

$\mathrm{Zu}$ diesem Zweck wurden die evolutionären Methoden, die genetischen Algorithmen und die evolutionäre Strategie für die notwendigen Optimierungsmaßnahmen verwendet. Die Funktionalität und Wirkungsweise wurde ausführlich innerhalb der ersten Kapitel vorgestellt.

Den evolutionären Methoden wurde eine effiziente Geometriedefinition aerodynamischer Konfigurationen zur Seite gestellt. Diese stützt sich ausschließlich auf analytisch definierte Kurven und Formen. Dadurch konnte der Parameterraum klein gehalten und für die evolutionären Optimierungsmethoden nutzbar gemacht werden.

Die Optimierungsmethode und die Geometriedefinition bringen für den transsonischen Geschwindigkeitsbereich offensichtlich sehr gute aerodynamische Ergebnisse hervor. Die Arbeit zeigt, welche fundamentale Bedeutung einer auf die Optimierungsmethode abgestimmte Geometriedefinition zukommt. Die in dieser Arbeit behandelten Konfigurationen sind in ihrer geometrischen Definition vergleichsweise einfach. Die Anzahl der zu optimierenden Komponenten einer flugfähigen Konfiguration ist natürlich sehr viel größer als hier angenommen. In der umfassenden Optimierung einer Konfiguration müßten alle diese Komponenten miteinbezogen werden, einschließlich der Bewertung interdisziplinärer Aspekte. Diese Arbeit stellt nur einen ersten Schritt in diese Richtung dar. Hinsichtlich der aerodynamischen Grundkomponeten (Profil und Tragflügel) eines Verkehrsflugzeuges ist dieser Schritt gelungen.

Der Endzustand der Optimierung ist mit der Paretofront erreicht. Die Objekte der Paretofront stellen für exakt einen Designpunkt eine optimale Konfiguration dar. Im transsonischen Bereich reagiert die Strömung sehr sensibel auf geometrische Veränderungen, so daß optimal gestaltete Konfigurationen wirklich nur für diesen einen Punkt der Paretofront optimal sind. Diese Tatsache verdeutlicht, welche Feinheiten in der Geometrie durch den genetischen Algorithmus angepaßt werden konnten. Die evolutionäre Strategie konnte darüber hinaus noch geringfügig feinere Anpassungen vornehmen. Sie konnte hier aber hinsichtlich Handhabung und Konvergenzgeschwindigkeit nicht grundsätzlich überzeugen.

Die in der Zielsetzung genannte angestrebte Grenze ist mit der Konvergenz der Objekte gegen die Paretofront erreicht. Mit den evolutionären Methoden konnte sie aufgespürt werden. Die Sichtung des Strömungsfeldes der Objekte auf der Paretofront zeigt, daß eine weitere aerodynamische Verbesserung der Objekte nicht ohne erweiterte geometrische Möglichkeiten zu realisieren ist. Die Geometrie der Objekte der Paretofront ist in den vorgegebenen geometrischen Grenzen optimal an die Strömungsverhältnisse angepasst. 
Der Verlauf der optimierten Geometrieparameter entlang der Paretofront gibt wichtige Hinweise auf die grundsätzlich benötigte Gestaltungsfreiheit von Konfigurationen im Bereich optimaler aerodynamischer Effizienz. Somit kann die Arbeit mit genetischen Algorithmen wesentlich zur Erweiterung der praktischen Wissensbasis beitragen.

Die evolutionären Methoden zeigen auch bei Anwendungen in der Aerodynamik ihr volles Potential. Sie stellen ihre Robustheit und Treffsicherheit unter Beweis. Für die hier vorkommenden Strömungsprobleme konnten optimale Anpassungen der Geometrie vorgenommen werden. Das konnte bei ebener Profilierung für reibungsfreie und reibungsbehaftete Strömungen sowie für die Tragflügelauslegung im Unter- und Überschall gezeigt werden.

In dieser Arbeit wurde eine einzige Zahl, das Verhältnis von Auftrieb zu Widerstand (L/D), optimiert. Bei Strömungsrechnungen mit dem Euler-Verfahren wurde nur der reibungsfreie (induzierter und Wellen-) Widerstand berücksichtigt. Die Beispiele haben gezeigt, daß die gewählten geometrischen Parameter sehr geeignet sind, diesen Anteil des Widerstandes zu minimieren.

In verfeinerten Untersuchungen unter Nutzung von Navier-Stokes-Verfahren wird auch der Reibungsanteil des Widerstandes minimiert. In zukünftigen Arbeiten legt es die aerodynamische Wissensbasis dann nahe, auch den Verlauf der Druckverteilungen entlang von Profilen und Flügelschnitten vorzuschreiben, um günstige Resultate für die Reibungseinflüsse zu erhalten. Diese inverse Komponente der Optimierung (weitgehende Vorgabe des Resultates der Umströmung und Auffinden einer mit dieser Strömung kompatiblen Geometrie) bildet eine attraktive Erweiterung des Anwendungsbereichs der hier vorgestellten Rechnungen mit GA.

Die Optimierung mit Hilfe evolutionärer Methoden ist sehr rechenintensiv. Aus diesem Grunde verbreitert sich das Anwendungsspektrum dieser Methoden parallel zur Rechnerentwicklung. Da die Computertechnologie innerhalb kürzester Zeit enorme Fortschritte macht, ist mit einer verstärkten Anwendung auch innerhalb der Strömungsmechanik in absehbarer Zeit zu rechnen. 


\section{Anhang A Basisfunktionen zur Geometriedefinition}

Zur Geometriedefinition werden zweidimensionale Kurven, aus denen auch die Flächen berechnet werden, aus verschiedenen Basisfunktionen zusammengesetzt. Dazu wird eine Kurve in disjunkte Intervalle unterteilt und für jedes Intervall eine Basiskurve angegeben. Die Basisfunktionen besitzen unterschiedliche geometrische Eigenschaften. Sie haben gemeinsam, daß sie auf dem Einheitsquadrat definiert sind. Die Parameter $\mathrm{s}_{1}, \mathrm{~s}_{2}, \mathrm{e}_{1}, \mathrm{e}_{2}$ kontrollieren den Kurvenverlauf der Funktionen und sind für jedes Intervall konstant. Die Parameter $\mathrm{s}_{1}, \mathrm{~s}_{2}$ dienen als Tangenten in den Endpunkten $(0,0)$ und $(1,1)$. Dieser Sachverhalt erlaubt auf einfache Weise die Erzeugung differenzierbarer Übergange an den Intervallgrenzen.

Die Basisfunktionen zeichnen sich durch einige gemeinsame Eigenschaften aus:

- Die Funktionen sind stetig.

- Die Punkte $(0,0)$ und $(1,1)$ werden interpoliert.

- Die Funktionen werden auf dem Einheitsintervall definiert.

- Die Funktionen sind monoton steigend.

- Die Tangentensteigungen an den Endpunkten sind durch die Parameter $\mathrm{s}_{1}$, $\mathrm{s}_{2}$ gegeben.

- Es werden maximal 4 Parameter pro Basisfunktion zur Kontrolle des Kurvenverlaufs benötigt (-> Optimierung).

Bei der Definition der Kurven wird der Graph der Basisfunktion vom Einheitsquadrat auf das entsprechende Intervall abgebildet. Dazu wird zu jeder Basisfunktion der Startpunkt $\left(\mathrm{x}_{1}, \mathrm{y}_{1}\right)$ und der Endpunkt $\left(\mathrm{x}_{2}, \mathrm{y}_{2}\right)$ auf der Kurve angegeben. Der Endpunkt eines Intervalls ist der Anfangspunkt des nächsten Intervalls, wodurch Stetigkeit der Kurven gegeben ist.

Die verwendeten Basisfunktionen werden intern über (meist) ganzzahlige Zahlen $g$ referenziert. Für jedes Intervall werden diese Nummern angegeben, um zu bestimmen, welche Funktion benutzt werden soll. Damit ist auch die Nutzung unterschiedlicher Funktionen innerhalb einer Kurve möglich. In dieser Arbeit wurde hiervon allerdings kein Gebrauch gemacht.

Insgesamt fallen somit bis zu neun Parameter pro Intervall an. Diese sind:

- $\left(\mathrm{x}_{1}, \mathrm{y}_{1}\right)$ - Startpunkt des Kurvenintervalls.

- $\left(\mathrm{x}_{2}, \mathrm{y}_{2}\right)$ - Endpunkt des Kurvenintervalls (wird nur für das letzte Intervall benötigt).

- g - Funktionsindex, dient zur internen Organisation.

- $\mathrm{s}_{1}$ - Tangentensteigung im Startpunkt.

- $\mathrm{s}_{2}$ - Tangentensteigung im Endpunkt.

- $\mathrm{e}_{1}$ - Unterschiedliche Bedeutung.

- $\mathrm{e}_{2}$ - Unterschiedliche Bedeutung 
Damit ist die Anzahl der freien Parameter sehr begrenzt. Durch die speziellen Eigenschaften der verschiedenen Funktionen lassen sich große Intervalle einer Kurve durch eine einzige Basisfunktion ausdrücken. Insgesamt reduziert sich dadurch die Zahl der Kontrollwerte. Innerhalb dieser Arbeit sind nur drei spezielle Funktionen verwendet worden.

\section{A.1 Cubic-Funktion}

Die folgende Funktion entspricht einem kubischen Polynom. Die Koeffizienten des Polynoms sind durch die vier Bedingungen (Werte und Tangentensteigung in $(0,0)$ und $(1,1)$ bestimmt. Die Parameter $\mathrm{e}_{1}$ und $\mathrm{e}_{2}$ werden nicht benutzt.

$$
\text { cubic: } g\left(s_{1}, s_{2} ; x\right)=s_{1} \cdot x+\left(-2 \cdot s_{1}-s_{2}+3\right) \cdot x^{2}+\left(s_{1}+s_{2}-2\right) \cdot x^{3}
$$

Die Koeffizienten des Polynoms waren gerade so gewählt, daß die Steigungen der Tangenten in 0 und 1 durch die Parameter $\mathrm{s}_{1}$ und $\mathrm{s}_{2}$ gegeben waren:

$$
\begin{aligned}
& \frac{d g}{d x}\left(s_{1}, s_{2} ; x\right)=s_{1}+2\left(-2 \cdot s_{1}-s_{2}+3\right) \cdot x+3\left(s_{1}+s_{2} \cdot 2\right) \cdot x^{2} \\
& \frac{d g}{d x}\left(s_{1}, s_{2} ; 0\right)=s_{1} \\
& \frac{d g}{d x}\left(s_{1}, s_{2} ; 1\right)=s_{2}
\end{aligned}
$$

Für die zweite Ableitung gilt:

$$
\begin{aligned}
& \frac{d^{2} g}{d x}\left(s_{1}, s_{2} ; x\right)=2\left(-2 \cdot s_{1}-s_{2}+3\right)+6\left(s_{1}+s_{2}-2\right) \cdot x \\
& \frac{d^{2} g}{d x}\left(s_{1}, s_{2} ; 0\right)=-4 s_{1}-2 s_{2}+6 \\
& \frac{d^{2} g}{d x}\left(s_{1}, s_{2} ; 1\right)=2 s_{1}+4 s_{2}-6
\end{aligned}
$$

Die kubische Funktion hat den Vorteil, daß sie die Krümmung minimiert. Um die Krümmung in beiden Eckpunkten verschwinden zu lassen, müssen beide Parameter auf 1 gesetzt werden. Dann erhält man aber nur eine Gerade. Ansonsten läßt sich eine verschwindende Krümmung nur an den Endpunkten realisieren. 


\section{A.2 Quintic-Funktion}

Analog zur kubischen Funktion ist diese Funktion ein Polynom vom Grad 5. Dies ermöglicht neben den Werten und Tangentensteigungen in $(0,0)$ und $(1,1)$ zusätzlich noch die Vorgabe der 2. Ableitung in diesen beiden Punkten. Damit sind die 6 Polynomkoeffizienten bestimmt.

$$
\text { quintic: } g\left(s_{1}, s_{2}, e_{1}, e_{2} ; x\right)=\sum_{i=0}^{5} a_{i} x^{i}
$$

mit:

$$
\begin{aligned}
& a_{0}=0 \\
& a_{1}=s_{1} \\
& a_{2}=\frac{1}{2} \cdot e_{1} \\
& a_{3}=10 \cdot\left(1-s_{1}-e_{1}\right)-4(1) \cdot\left(s_{2}-s_{1}-2 \cdot e_{1}\right)+\frac{1}{2}\left(e_{2}-e_{1}\right) \\
& a_{4}=15 \cdot\left(1-s_{1}-e_{1}\right)+7 \cdot\left(s_{2}-s_{1}-2 \cdot e_{1}\right)-\left(e_{2}-e_{1}\right) \\
& a_{5}=6 \cdot\left(1-s_{1}-e_{1}\right)-3 \cdot\left(s_{2}-s_{1}-2 \cdot e_{1}\right)+\frac{1}{2}\left(e_{2}-e_{1}\right) \\
& \frac{d}{d x} g\left(s_{1}, s_{2}, e_{1}, e_{2} ; x\right)=\sum_{i=1}^{5} i a_{i} x^{i-1} \\
& \frac{d}{d x} g\left(s_{1}, s_{2}, e_{1}, e_{2} ; 0\right)=s_{1} \\
& \frac{d}{d x} g\left(s_{1}, s_{2}, e_{1}, e_{2} ; 1\right)=s_{2} \\
& \frac{d^{2}}{d x} g\left(s_{1}, s_{2}, e_{1}, e_{2} ; x\right)=\sum_{i=2}^{5} i(i-1) a_{i} x^{i-2} \\
& \frac{d^{2}}{d x} g\left(s_{1}, s_{2}, e_{1}, e_{2} ; 0\right)=e_{1} \\
& \frac{d^{2}}{d x} g\left(s_{1}, s_{2}, e_{1}, e_{2} ; 1\right)=e_{2}
\end{aligned}
$$


Mit der Quintic-Funktion hat man die Möglichkeit, das Krümmungsverhalten an den Übergängen zu kontrollieren. Setzt man die Parameter $\mathrm{e}_{1}$ und $\mathrm{e}_{2}$ auf Null, so entsteht eine Kurve mit einer in den Randpunkten verschwindenden Krümmung.

\section{A.3 Ramp-Funktion}

Für eine Ramp-Funktion werden die vier Parameter $\mathrm{s}_{1}, \mathrm{~s}_{2}, \mathrm{e}_{1}, \mathrm{e}_{2}$ benötigt. Die Parameter $\mathrm{e}_{1}$ und $\mathrm{e}_{2}$ geben hier Exponenten an. Für ganzzahlige Parameter $\mathrm{e}_{1}$ und $\mathrm{e}_{2}$ sind die Ramp-Funktionen Polynome. Die Parameter $e_{1}$ und $e_{2}$ müssen positiv sein, damit sie auf dem Einheitsintervall definiert sind. Die Tangentensteigungen $s_{1}$ und $s_{2}$ werden nicht angenommen, wenn $e_{1}$ oder $e_{2}$ kleiner gleich 1 sind. Die durch $s_{1}$ und $s_{2}$ festgelegten Tangenten dürfen sich nicht innerhalb des Einheitsquadrats schneiden. D.h. es gilt: $\mathrm{s}_{1}<1=0<\mathrm{s}_{2}<1$ oder $\mathrm{s}_{1}>1=>\mathrm{s}_{2}>1$. Außerdem gilt $s_{1}=1 \Rightarrow s_{2}=1$.

$$
\begin{aligned}
& g_{0}\left(s_{1}, s_{2}, e_{1}, e_{2} ; x\right)=s_{1} \cdot x+\left(\left(s_{2}-s_{1}\right) \cdot x+1-s_{2}\right) \cdot\left(1-\left(1-x^{e_{1}}\right)^{e_{2}}\right) \\
& g_{1}\left(s_{1}, s_{2}, e_{1}, e_{2} ; x\right)=s_{1} \cdot x+\left(\left(s_{2}-s_{1}\right) \cdot x+1-s_{2}\right) \cdot\left(1-(1-x)^{e_{2}}\right)^{e_{1}}
\end{aligned}
$$

Außer dem beiden angegebenen Ramp-Funktionen 0 und 1 kann man auch eine Kombination beider Funktionen auswählen, wobei der Funktionsindex $c$ wie ein Parameter agiert. Der Index $c$ gibt das Mischverhältnis der beiden Ramp-Funktionen $g_{0}$ und $g_{1}$ an.

$$
\text { ramp: } g_{c}\left(s_{1}, s_{2}, e_{1}, e_{2}\right)=c g_{0}\left(s_{1}, s_{2}, e_{1}, e_{2}\right)(x)+(1-c) g_{1}\left(s_{1}, s_{2}, e_{1}, e_{2}\right)(x)
$$

mit $c \in[0,1]$.

Für die Ableitungen gilt:

$$
\begin{aligned}
& \frac{d}{d x} g_{0}\left(s_{1}, s_{2}, e_{1}, e_{2} ; x\right)=s_{1}+\left(s_{2}-s_{1}\right)\left(1-\left(1-x{ }^{e_{1}}\right)^{e_{2}}\right)+e_{1} e_{2}\left(1-s_{2}+\left(s_{2}-s_{1}\right) x\right)\left(1-x{ }^{e_{1}}\right)^{e_{2}-1}{ }_{x}^{e_{1}-1} \\
& \frac{d}{d x} g_{1}\left(s_{1}, s_{2}, e_{1}, e_{2} ; x\right)=s_{1}+\left(s_{2}-s_{1}\right)\left(1-(1-x)^{e_{2}}\right)^{e_{1}}+e_{1} e_{2}\left(1-(1-x)^{e_{2}}\right)^{e_{1}-1}(1-x)^{e_{2}-1}\left(1-b+\left(s_{2}-s_{1}\right) \cdot x\right)
\end{aligned}
$$

Wie man aus der Ableitung ersieht, entsprechen die Parameter $\mathrm{s}_{1}>0$ und $\mathrm{s}_{2}>0$ für $\mathrm{e}_{1}>1$ und $\mathrm{e}_{2}>1$ den Steigungen der Kurve in den Endpunkten. Der Graph der Ramp-Funktion liegt für hinreichend große $\mathrm{e}_{1}$ und $\mathrm{e}_{2}$ zwischen den beiden Geraden $g_{1}(x)=s_{1} x$ und $g_{2}(x)=1-b+b x$. Sind $\mathrm{e}_{1}$ und $\mathrm{e}_{2}$ kleiner gleich 1 , so folgt der Kurvenverlauf nicht den beiden Tangenten. Stattdessen geht die 1. Ableitung für $\mathrm{e}_{1}<1$ im Ursprung und für $\mathrm{e}_{2}<1$ in $(1,1)$ gegen unendlich. 


\section{Literaturverzeichnis}

\section{[1] Ablay P.}

Optimieren mit Evolutionsstrategien.

Spektrum der Wissenschaft, S. 104-155, 1987.

[2] Beasley, D., Bull, D. R., Martin, Ralph R.

An Overview of Genetic Algorithms. Part 1, Fundamentals.

University Computing 1993 15(2) 58-69.

[3] Beasley, D., Bull, D. R., Martin, Ralph R.

An Overview of Genetic Algorithms. Part 2, Research Topics.

University Computing 1993 15(4) 170-181.

[4] Buckles, Bill P. and Petry, Frederick E.

Genetic Algorithms.

IEEE Computer Society Press, Los Alamitos, California 1992.

\section{[5] Davis L.}

Handbook of Genetic Algorithm.

Van Nostrand Reinhold, 1991.

[6] De Jong, K. A.

A genetic-based global function optimization techniques.

(Tech. Rep. No. 80-2). Pittsburgh, PA, 1980.

\section{[7] DLR e.V.}

FLOWer.

Installation and User Handbook. Deutsche Forschungsanstalt für Luft- und Raumfahrt e.V. Doc.-Nr. MEGAFLOW-1001, 1997.

[8] Dulikravich, G.S., Mertens, J., Seebass, R., Sobieczky, H., Van der Velden, A. New Design Concepts For High Speed Air Transport.

CISM Courses and Lectures NO. 366. Springer, Wien - NewYork, 1997.

[9] Gerhold, T., Galle, M., Friedrich, O., Evans, J.

Calculation of Complex Three Dimensional Configurations Employing the DLR-TAUCode. AIAA paper 97-0167, 1997.

[10] Goldberg, David E.

Real-coded Genetic Algorithms, Virtual Alphabets, and Blocking.

(IlliGAL Report No. 90001). Urbana, IL, 1990. 
[11] Goldberg, David E.

Genetic Algorithms in Search, Optimization \& Maschine Learning.

Addison-Wesley, MA, 1989.

[12] Goldberg, David E.

Genetic Algorithms.

New York: McGraw-Hill, 1995.

[13] Heistermann, Jochen

Genetische Algorithmen. Teubner-Texte zur Informatik, Band 9, 1992.

[14] Holland, John H.

Adaption in Natural and Artifical Systems.

Ann Arbor: The University of Michigan Press, 1975.

[15] Hyoung-Jin, Kim and Oh-Hyun, Rho.

Aerodynamic Design of Transonic Wing Using the Target Pressure Optimization Approach. AIAA 98-0599.

[16] Jeong, S., Matsushima, K., Iwamiya, T., Obayashi, S., Nakahashi, K. Inverse Design Method for Wings of Supersonic Transport.

AIAA 98-0602.

[17] Jones, Robert T.

Wing Theory.

Princeton University Press, Oxford, UK, 1990.

[18] Klein, M. \& Sobieczky, H.

Tragflügelgeometrie mit analytisch definierter Profilierung.

DLR, Techn. Note H100D-12.94.

[19] Kupier, H., van der Wees, A.J., Hendriks, C.F.W. and Labrujere, Th.E Application of Genetic Algorithms to the Design of Airfoil Pressure Distributions. National Aerospace Laboratory NLR, The Netherlands.

[20] Li, P., Seebass R., Sobieczky H.

Manual Aerodynamic Optimization of an Oblique Flying Wing.

AIAA-98-0598.

[21] Li, P., Sobieczky H., Seebass R.

A Design Method for Supersonic Transport Wings.

University of Colorado, Boulder and DLR, Göttingen. Tech. Note H116-04.95.

[22] Mantel, B., Periaux, J., Sefrioui, M., and Stouffelt, B.

Evolutionary Design in Aerodynamics.

AIAA 98-0222. 
[23] Nadarajah, S. and Jameson, A.

A Comparison of the Continuous and Discrete Adjoint Approch to Automatic Aerodynamic Optimization.

AIAA-2000-0667.

[24] Quagliarella D. , Periaux J. ,Poloni C. , Winter G.

Genetic Algorithms and Evolution Strategies in Engeneering and Computer Science.

John Wiley \& Sons, Chichester, 1998.

[25] Obayashi, S.

Genetic Algorithm of Target Pressure Distributions for Invers Design Methodes.

12 th CFD Conference, AIAA 95-1649, USA, 1995.

[26] Obayashi, S., Oyama, A.

Three-dimensional Aerodynamic Optimization with Genetic Algorithm.

Proceedings of the Third ECCOMAS Computational Fluid Dynamics Conference, Paris, John Wiley \& Sons Ltd, Chichester, September 9-13, 1996.

[27] Obayashi, S., Nakahashi, K., Oyama, A. and Yoshino, N.

Design Optimization of Supersonic Wings Using Evolutionary Algorithms.

Proceedings of the Fourth ECCOMAS Computational Fluid Dynamics Conference,

Athens, John Wiley \& Sons Ltd, Chichester, September 7-11, 1998.

[28] Oswatitsch, K.

Spezialgebiete der Gasdynamik.

Springer, Wien - New York, 1977.

[29] Oyama, A., Obayashi, S., Nakahashi K.

Transsonic Wing Optimization Using Genetic Algorithm.

AIAA 97-1854.

[30] Oyama, A., Obayashi, S., Nakahashi K., and Nakamura, T.

Euler/Navier-Stokes Optimization of Supersonic Wing Design Based on

Evolutionary Algorithm. Proceedings of the 10th International Conference on Parallel

CFD, Hsinchu, May 11-14, 1998.

[31] Oyama, A., Obayashi, S., Nakahashi K. and Naoki Hirose

Fractional Factorial Design of Genetic Coding For Aerodynamic Optimization.

AIAA-99-3298.

[32] Oyama, A., Obayashi, S., Nakahashi K.

Wing Design Using Real-Coded Adaptive Range Genetic Algorithm.

Proceedings of 1999 IEEE International Conference on Systems, Man, and Cybernetics,

Tokyo, Japan, October 12-15, 1999. 
[33] Pagendarm, H.-G.

HIGHEND, A Visualization System for 3D Data with Special Support for Postprocessing of Fluid Dynamics Data.

in: M. Grave, Y. LelLous, W.T. Hewitt (ed.) Visualization in Scientific Computing, pp.87-98, pp. 199-200, Springer, 1994

[34] Pagendarm, H.-G.

Scientific Visualization in Computational Fluid Dynamics.

in: Visualization and Intelligent Design in Engineering and Architecture (eds. Connors, J.J., Hernandez, S., Murthy, T.K.S., Power, H.), Computational Mechanics Publications, Elsevier Science Publishers, Essex, UK (1993).

[35] Rechenberg, I.

Evolutionsstrategie.

Friedrich Frommann Verlag, Stuttgart, 1973.

[36] Schlichting, H., Truckenbrodt, E.

Aerodynamik des Flugzeuges.

Springer-Verlag Berlin Heidelberg New York 1969.

[37] Schöneburg, E., Heinzmann, F., Feddersen, S.

Genetische Algorithmen und Evolutionstrategien.

Addison-Wesley, 1.Auflage 1994.

[38] Schwefel, H.-P.

Numerische Optimierung von Computer-Modellen mittels der Evolutionsstrategie.

Birkhäuser Verlag, Basel, 1977.

[39] Sobieczky, H., Zhu, Z.

Transonic Wing Design: Off Design Efficiency and Effect of Fictitious Gas Parameter. DLR-IB 221-85 A 24, 1985.

[40] Sobieczky, H.

The Combination of a Geometry Generator with Transonic Design and Analysis Algorithms.

AIAA 83-1862, 1983.

[41] Sobieczky, H.

Verfahren für die Entwurfsaerodynamik moderner Transportflugzeuge.

DLR-FB-85-43, 1985.

[42] Sobieczky, H., Seebass, R, Li, P., Hannemann, M.

Manual Aerodynamic Optimization of Oblique Flying Wing.

ICAS-98-2.2.3. 21st Congress of the International Council of the Aeronautical Sciences, Melbourne, Australia. 


\section{[43] Sobieczky, H.}

Parametric Airfoils and Wings.

In: K. Fuji and G. S. Dulikravich (Eds.): Notes on Numerical Fluid Mechanics, Vol. 68, Wiesbaden: Vieweg, 1998. Tech. Note H150B-98.11, 1998.

[44] Soto, O., Löhner, R.

CFD Optimization Using an Incomplete-Gradient Adjoint Approach. AIAA-2000-0666.

\section{[45] Stuff, R.}

Optimierungsverfahren Evolutionsstrategie zur Lösung der Forschungsaufgaben der DFVLR, IB 252 - 80 A 08, 1980.

[46] Takahashi, S., Obayashi, S. and Nakahashi, K.

Inverse Optimization of Transonic Wing shape for Mid-Size Regional Aircraft. AIAA 98-0601.

[47] Yamamoto, K. and Inoue, $O$.

Applications of Genetic Algorithm to Aerodynamic Shape Optimization. AIAA 95-1650, 1995. 


\section{Danksagung}

Die vorliegende Arbeit entstand während meiner Tätigkeit als Mitarbeiter am Institut für Strömungsmechanik des Deutschen Zentrums für Luft- und Raumfahrt (DLR) in Göttingen.

An dieser Stelle möchte ich ganz besonders Herrn Professor Dr. techn. habil. H. Sobieczky für seine hervorragende Unterstützung danken. Mit seinem fördernden Interesse und wertvollen Anregungen hat er im wesentlichen zum Gelingen der Arbeit beigetragen.

Herrn Prof. Dr. E.A. Müller danke ich für die Betreuung und Übernahme der Verantwortung einer interdisziplinären Arbeit im Grenzbereich der Strömungsphysik, Informatik und Numerik. Seine interessanten Ausführungen im Gebiet der Strömungsmechanik haben bei mir die Begeisterung für die transsonische Aerodynamik und Gasdynamik geweckt.

Bei der grafischen Analyse meiner Ergebnisse wurde ich durch Frau Monika Hannemann und Frau Birgit Henne durch ihr großes Wissen um Auswertesysteme stets gut beraten. Insbesondere Frau Monika Hannemann danke ich für wertvolle Hinweise und Anregungen.

Schließlich möchte ich mich bei meiner Lebensgefährtin Frau Stephanie Alberti für die unendliche Geduld und Sorge um mein Wohlergehen ganz besonders bedanken. 